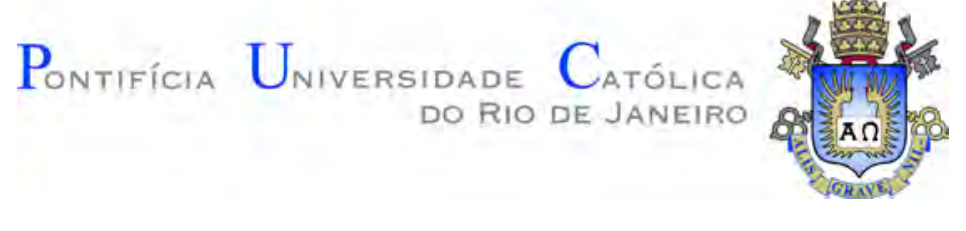

Syed Hamza Safeer Gardezi

\title{
Atomically Thin Semiconducting Transition-Metal Dichalcogenides: From Synthesis to Electro-Optical Properties
}

Tese de Doutorado

Thesis presented to the Programa de Pós-graduação em Física of PUC-Rio in partial fulfillment of the requirements for the degree of Doutor em Ciências - Física.

Advisor: Prof. Victor Carôzo Gois de Oliveira 


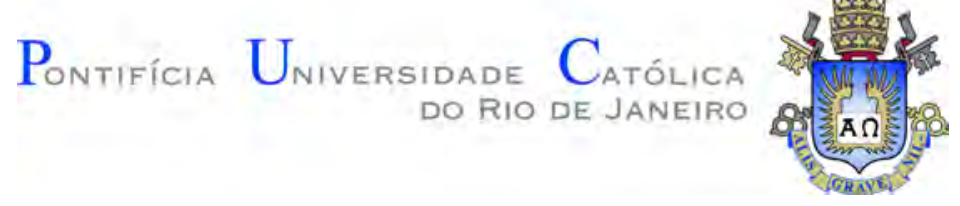

Syed Hamza Safeer Gardezi

\begin{abstract}
Atomically Thin Semiconducting Transition-Metal Dichalcogenides: From Synthesis to Electro-Optical Properties
\end{abstract}

Thesis presented to the Programa de Pós-graduação em Física of PUC-Rio in partial fulfillment of the requirements for the degree of Doutor em Ciências - Física. Approved by the Examination Committee:

\author{
Prof. Victor Carôzo Gois de Oliveira \\ Advisor \\ Departamento de Física - PUC-Rio \\ Prof. Fernando Lázaro Freire Junior \\ Departamento de Física - PUC-Rio \\ Prof. Isabel Cristina dos Santos Carvalho \\ Departamento de Física - PUC-Rio
}

Prof. Cristiano Fantini Leite

UFMG

Prof. Bruno Ricardo de Carvalho

UFRN

Prof. Daniel Grasseschi

UFRJ

Rio de Janeiro, September 29th, 2020 
All rights reserved. Total or partial reproduction of this work is forbidden without the authorization of the university, the author, and the advisor.

\section{Syed Hamza Safeer Gardezi}

Obtained his Master's degree in Physics from Quaid-i-Azam University, Islamabad, Pakistan.

Bibliographic data

Syed Hamza Safeer Gardezi

Atomically Thin Semiconducting Transition-Metal Dichalcogenides: From Synthesis to Electro-Optical Properties / Syed Hamza Safeer Gardezi; advisor: Victor Carôzo Gois de Oliveira. - Rio de janeiro: PUC-Rio, Departamento de Física, 2020.

v., $141 \mathrm{f:} \mathrm{il.} \mathrm{color.} \mathrm{;} 30 \mathrm{~cm}$

Tese (doutorado) - Pontifícia Universidade Católica do Rio de Janeiro, Departamento de Física.

Inclui bibliografia

1. Informática - Teses. 2. Dicalcogenetos de metais de transição;. 3. APCVD;. 4. Síntese;. 5. Raman;. 6. Fotoluminescência; . 7. Cálculos de primeiros princípios;. 8. Geração de segundo harmônico;. 9. óptica não linear;. 10. Defeitos;. 11. Dispositivos..

I. Victor Carôzo Gois de Oliveira. II. Pontifícia Universidade Católica do Rio de Janeiro. Departamento de Física. III. Título. 

encouragement and sacrifices. 


\section{Acknowledgments}

(All praises to ALLAH ALMIGHTY, The Creator and Sustainer of The Universe, The most gracious, The invincible and the all-wise. All esteem to His Holy Prophet Muhammad (Peace be upon Him) for eternal source of guidance and knowledge for mankind.)

This work would not have been possible without the help and support of many people, and I hereby would like to take the opportunity to thank them. First of all, my supervisor Prof. Victor Carozo. His enthusiasm for my project and his constant belief in my abilities have been an invaluable support. He always encouraged my ideas and gave me the freedom and financial framework to realize them.

I would like to thank Prof. Fernando Lazaro head of our group and Prof. Marcelo da Costa for allowing me to work in their laboratory and added their scientific knowledge from time to time to make our work better and successful.

My colleagues at Van de Graaff Laboratory, most notably André do Nascimento, Zhang Shuai, Antônio Federico, Ceser Diaz, Neileth Stand, Cintia Aparecida, Nilton and, Suellen Tozetti, who helped by raising questions and having conversations to create a pleasant working environment. I enjoyed my discussions with them, and thanks to Ceser Diaz for doing SEM on our sample.

I extend a special thank you to Michael Nazarkovsky from Chemistry department for collaborating with us in a project and helping me in understanding the chemical aspects of my work.

I would like to thank Prof. Christiano de Matos from Mack Graphe, Mackenzie University, S.P, for collaboration on the non-linear optical properties of our samples, and allowing me to work in his laboratory. Prof. Isabel Carvalho who gave the idea to work on the non-linear properties. I acknowledge the use of the Mack Graphe, Mackenzie University, S.P. and special thanks to Alaxandre Samuel who measured the SHG for us.

I would like to thank Prof. Marcus Moutinho from Núcleo Multidisciplinar de Pesquisas em Computação NUMPEX-COMP, Campus Duque de Caxias, UFRJ, who performed the first-principle calculations.

I acknowledge the use of the Brazilian National Laboratory of Nanoscience and Nanotechnology (LABNANO/CBPF). I acknowledge the use of the XFlow Chemistry and Nanochemistry Lab, PUC-Rio for Raman measurements. This study was financed in part by the Coordenação de Aperfeiçoaento de Pessoal de Nível Superior - Brasil (CAPES) - Finance Code 001. I acknowledge support from CNPq grant numbers 304144/2018-5 and 429773/2018-8. 
Thank you to my Pakistani friends who give me the backup all the time, not a single moment I felt that I am away from my family, namely Adnan Raza, Rafqat Ali, Quaid Zaman, Tahir Jan, Asmat Pervaiz, Khalil ur Rehman and Rafi Khan.

And most of all, my family in Pakistan. I cannot express in words how grateful I am for your understanding and encouragement, and the constant love and support you have given me during all my life. Especially, I would like to thank my mother, who always pray for me back home and I thought its because of her prayers I achieve success.

Finally, thank you to the Brazilian government for giving me the opportunity to study in such a beautiful country. I experienced no discrimination here, Brazilian people are very friendly and hospitable, wherever I traveled in Brazil. I will not forget the time I spent here, my entire life. 


\section{Abstract}

Syed Hamza Safeer Gardezi; Victor Carôzo Gois de Oliveira (Advisor). Atomically Thin Semiconducting Transition-Metal Dichalcogenides: From Synthesis to Electro-Optical Properties. Rio de Janeiro, 2020. 141p. Tese de doutorado - Departamento de Física, Pontifícia Universidade Católica do Rio de Janeiro.

The aim of this work was to develop reliable and repeatable methods for growing high-quality monolayer $\mathrm{MoS}_{2}, \mathrm{WS}_{2}$, and their vertical heterostructure by atmospheric pressure chemical vapor deposition (APCVD) technique. The monolayer of these materials have vital importance in the fabrication of new optical and nanoelectronic devices. Thin and low-cost devices have increased the demand for new synthesis processes. Usually, the synthesis requires temperatures around $800^{\circ} \mathrm{C}$, which is an issue for applications mentioned above. In this thesis, we propose a new route using the APCVD technique to grow monolayers of $\mathrm{MoS}_{2}$ at $550^{\circ} \mathrm{C}$ mediated by sodium as a catalyst. We have produced single crystals and polycrystals by controlling the $\mathrm{NaNO}_{3} / \mathrm{MoO}_{3}$ precursor's ratio and growth time. Using first-principles calculations, we find out that sodium is the nucleation site of the growth process. The precursor's ratio is crucial to decrease the energy formation and the synthesis temperature. Firstprinciples calculations and experiments agree with the ideal precursor's rate of 0.3 and with the decrease of the synthesis temperature of $250^{\circ} \mathrm{C}$. We investigated the CVD grown sample with X-ray photoelectron spectroscopy, atomic force microscopy, Raman spectroscopy, photoluminescence spectroscopy, and transport experiments.

Few layers of TMDs allow us to create new materials and find new physical phenomena. The stacking sequence in few-layer TMDs can significantly impact on their electrical and optical properties. We also synthesized few layers of $\mathrm{MoS}_{2}$ and $\mathrm{WS}_{2}$ via APCVD. Two and three layers of $\mathrm{MoS}_{2}, \mathrm{WS}_{2}$, and their vertical heterostructures were characterized by second harmonic generation (SHG). The SHG shows that the layers in bilayers grow with $0^{\circ}$ or $60^{\circ}$ has different phase stacking. The SHG from $0^{\circ}$ stacked bilayer has increased when compared to monolayer, while the generated signal from bilayer with $60^{\circ}$ stacking is zero. This behavior of SHG suggests that the two layers of $\mathrm{MoS}_{2}$ or $\mathrm{WS}_{2}$ when stacked at $0^{\circ}$ have no inversion symmetry to $3 \mathrm{R}(\mathrm{AB})$ phase stacking between the top layer and the bottom layer. While when stacked with $60^{\circ}$ has inversion symmetry (Centrosymmetric) and have $2 \mathrm{H}\left(\mathrm{AA}^{\prime}\right)$ phase stacking.

Finally, the devices were fabricated on good quality samples to investigate their electrical performance. The fabricated devices show typical n-type 
behavior and mobility was estimated by measuring transport curves. The dependence of Raman modes of our heterostructure device with electron doping was also studied. By applying a voltage across our device the $\mathrm{A}_{1}^{\prime}$ mode shows blueshift and a new mode emerges at $\sim 410 \mathrm{~cm}^{-1}$, which is attributed to the defects (D) in the crystal.

\section{Keywords}

Transition metal dichalcogenide; APCVD; Synthesis; Raman; Photoluminescence; First-principles calculations; Second harmonic generation; Nonlinear optics; Defects; Devices. 


\section{Resumo}

Syed Hamza Safeer Gardezi; Victor Carôzo Gois de Oliveira. Dichalcogenetos de metal de transição semicondutores atomicamente finos: da síntese às propriedades eletro-ópticas. Rio de Janeiro, 2020. 141p. df de Doutorado - Departamento de Física, Pontifícia Universidade Católica do Rio de Janeiro.

O objetivo deste trabalho foi desenvolver métodos eficientes e reprodutíveis de crescimento de monocamadas de $\mathrm{WS}_{2}, \mathrm{MoS}_{2}$ e outras heteroestruturas verticais por deposição química em fase de vapor à pressão atmosférica (APCVD). A monocamada separada destes materiais tem grande importância na fabricação de novos dispositivos óticos e Nano eletrônicos. Dispositivos finos e de baixo custo necessitam temperaturas em torno de $800^{\circ} \mathrm{C}$, o que é um problema para aplicações mencionadas acima. Nesta tese, nós propusemos uma nova rota usando APCVD para crescer monocamadas de $\mathrm{MoS}_{2}$ a $550^{\circ} \mathrm{C}$, usando sódio como catalisador. Nós produzimos monocristais e poli cristais controlando a razão de precursores $\mathrm{NaNO}_{3} / \mathrm{MoO}_{3}$ e tempo de crescimento. Usando cálculos de primeiros princípios, mostramos que o sódio atua como centro de nucleação para o processo de síntese. A razão de precursores é crucial para diminuir a energia de formação e a temperatura de síntese. Cálculos de primeiros princípios e experimentos concordam que uma razão ideal é em torno de 0.3 , proporcionando uma queda de $250^{\circ} \mathrm{C}$ na temperatura de crescimento. Nós investigamos as amostras crescidas por APCVD usando espectroscopia de fotoelétrons induzidos por raios-X, microscopia de força atômica, espectroscopia Raman, fotoluminescência e mediadas de transporte.

Dicalcogenetos de metais de transição (TMD) dispostos em poucas camadas permitem-nos criar materiais e estudar novos fenômenos físicos. A sequência de empilhamento dos TMDs pode modificar suas propriedades opticas e elétricas. Também sintetizamos poucas camadas de $\mathrm{MoS}_{2}$ e $\mathrm{WS}_{2}$ usando APCVD. Duas e três camadas de $\mathrm{WS}_{2}, \mathrm{MoS}_{2}$ e suas heteroestruturas verticais foram caracterizadas através de geração de segundo harmônico (SHG). SHG mostra que as bicamadas crescidas com ângulos de rotação relativos de $0^{\circ}$ e $60^{\circ}$ possuem diferentes fases de empilhamento. O SHG do empilhamento bicamada com ângulo relativo de $0^{\circ}$ aumentos, enquanto para amostras com empilhamento de $60^{\circ}$ foi zerado. Este comportamento do SHG sugere que duas camadas de $\mathrm{MoS}_{2}$ ou $\mathrm{WS}_{2}$, quando empilhados a $0^{\circ}$ não possuem simetria de inversão para $3 \mathrm{R}(\mathrm{AB})$ entre as camadas inferiores e superiores, enquanto as camadas de $60^{\circ}$ possuem simetria de inversão (centrossimétricas) e possuem empilhamento na forma $2 \mathrm{H}(\mathrm{AA})$. 
Finalmente, dispositivos foram fabricados em amostras de boa qualidade para a investigação de sua performance elétrica. Os dispositivos mostram comportamento típico tipo-n e sua mobilidade foi estimada a partir das curvas de transporte. A dependência dos modos Raman das nossas amostras de heteroestruturas também foi estudada. Aplicando uma tensão nos dispositivos, o modo $\mathrm{A}_{1}^{\prime}$ mostrou um desvio para o azul e um novo modo surge em $410 \mathrm{~cm}^{-1}$, atribuídos defeitos (D) no cristal.

\section{Palavras-chave}

Dicalcogenetos de metais de transição; APCVD; Síntese; Raman; Fotoluminescência; Cálculos de primeiros princípios; Geração de segundo harmônico; óptica não linear; Defeitos; Dispositivos. 


\section{Table of contents}

1 Introduction $\quad 22$

2 Properties of Transition Metal Dichalcogenides $\quad 27$

2.1 Introduction to Transition Metal Dichalcogenides 27

$\begin{array}{lll}2.1 .1 & \text { Structure of TMDs } & 28\end{array}$

2.1.2 Stacking Sequences of Two Layers 29

2.2 Phonon Dispersion 31

2.3 Electronic Dispersion $\quad 32$

2.4 Linear Optical Spectroscopy 34

2.4.1 Photoluminescence in TMDs 34

2.4.2 Raman Modes in TMDs 36

2.5 Non-Linear Optical Spectroscopy 38

$\begin{array}{lll}2.6 & \text { Photoelectron Spectroscopy } & 39\end{array}$

3 Experimental Methodology $\quad 41$

3.1 Chemical Vapor Deposition 41

3.1.1 Preparation of the Substrate 43

3.1.2 Precursors and Crucible 43

3.1.3 Growth Process 43

3.1.4 Synthesis of Bilayers $\mathrm{MoS}_{2}, \mathrm{WS}_{2}$, and $\mathrm{MoS}_{2} / \mathrm{WS}_{2}$ Vertical Heterostructures $\quad 45$

3.2 Optical Microscope 46

3.3 Scanning Electron Microscope 47

3.4 Raman Spectroscopy 49

$\begin{array}{lll}\text { 3.4.1 Raman Effect } & 49\end{array}$

3.4.2 Theory of Raman Scattering $\quad 50$

3.4.3 Raman Spectrometer 51

3.5 Photoluminescence Spectroscopy 53

3.6 Atomic Force Microscopy 55

3.7 X-ray Photoelectron Spectroscopy 56

3.8 Introduction to Second Harmonic Generation 58

3.8.1 Second Harmonic Generation Measurements $\quad 59$

3.9 Device Fabrication 61

3.9.1 Resist Coating $\quad 62$

3.9.2 Lithography 62

$\begin{array}{ll}3.9 .3 & \text { Development }\end{array}$

$\begin{array}{ll}3.9 .4 & \text { Metal deposition }\end{array}$

3.9.5 Lift off 66

3.10 Electrical Measurements $\quad 67$

3.10.1 Electro-Raman $\quad 68$

$4 \quad$ CVD Grown $\mathrm{MoS}_{2}, \mathrm{WS}_{2}$ Monolayer, and their Vertical Heterostructure $\quad \mathbf{6 9}$

$\begin{array}{lll}4.1 \text { Introduction } & 69\end{array}$

4.2 $\mathrm{MoS}_{2}$ and $\mathrm{WS}_{2}$ Monolayer $\quad 69$ 
4.3 $\mathrm{MoS}_{2} / \mathrm{WS}_{2}$ Vertical Heterosctructure 74

4.4 Sodium Mediated Low Temperature Synthesis of Monolayers of Molybdenum Disulfide $\quad 75$

4.4.1 Catalytic Behaviour of Na Explained by DFT Calculations 79

4.4.2 Chemical Equations Explaining the Growth 82

4.4.3 Characterization of Na Mediated Synthesized Samples 82

4.5 Synthesis of $\mathrm{MoS}_{2}$ on Glass 86

4.6 Partial Conclusion 88

5 Second Harmonic Generation in Two and Three Layers of Transition Metal Dichalcogenides $\quad 90$

$\begin{array}{lll}5.1 \text { Introduction } & 90\end{array}$

5.2 Two and Three Layers of Transition Metal Dichalcogenides 90

5.3 Raman and Photoluminescence of Trilayer Samples 92

5.4 Second Harmonic Generation in Two and Three Layers of $\mathrm{MoS}_{2}, \mathrm{WS}_{2}$ with Different Stacking Orientation 95

5.4.1 SHG in $\mathrm{MoS}_{2} / \mathrm{WS}_{2}$ Heterostructure with Different Stacking Orientation 101

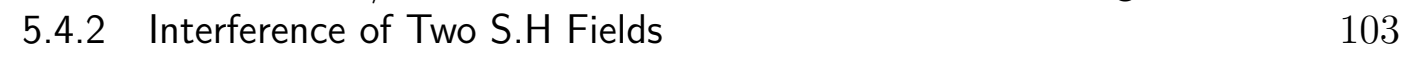

$\begin{array}{ll}5.5 \text { Partial Conclusion } & 104\end{array}$

6 Electrical Measurements $\quad 106$

$\begin{array}{lll}6.1 & \text { Introduction } & 106\end{array}$

$\begin{array}{ll}6.2 \text { Field Effect Transistor } & 107\end{array}$

$\begin{array}{lll}\text { 6.2.1 Operating Principle of FETs } & 107\end{array}$

$\begin{array}{lll}6.3 \text { Device Structure } & 108\end{array}$

$\begin{array}{lll}6.4 & \text { Transport Measurement of } \mathrm{MoS}_{2} & 109\end{array}$

6.5 Transport Measurement of $\mathrm{WS}_{2} \quad 111$

6.6 Transport Measurement of $\mathrm{MoS}_{2} / \mathrm{WS}_{2}$ Heterostructure 112

6.7 Defect Related Raman Peak Reveled by Electo-Raman of $\mathrm{MoS}_{2} / \mathrm{WS}_{2}$ Device 113

$\begin{array}{ll}\text { 6.8 Partial Conclusion } & 116\end{array}$

7 Conclusions and Future Work $\quad 117$

$\begin{array}{ll}\text { Bibliography } & 120\end{array}$ 


\section{List of figures}

Figure 2.1 Periodic table and different TMDs.

Figure 2.2 (a) 3D representation of the layered structure of transition metal dichalcogenides (TMDs). Adopted from [63]. (b) Archetypal coordination in TMDs. Octahedral coordination (left) triangular prismatic coordination (right) and top view of monolayers constructed from octahedral (left) and triangular prismatic (right) coordination. Adopted from [86].

Figure 2.3 Side and top views of (a) $2 \mathrm{H}$ stacking pattern of bilayer $\mathrm{MX}_{2}, 2 \mathrm{H}$ is the most stable stacking pattern (also named as $\mathrm{AA}^{\prime}$ stacking)(b) 3R (also named as AB stacking)

Figure 2.4 (a) Phonon dispersion curve of monolayer $\mathrm{MoS}_{2}$ calculated by A.Molina, et al. [90]. (b) and $\mathrm{WS}_{2}$.

Figure 2.5 Electronic band structures of (a) bulk $\mathrm{MoS}_{2}$ and, (b) monolayer $\mathrm{MoS}_{2}$ calculated from first principles using DFT-GGA approach. Valence band maxima and conduction band minima are indicated by red and blue circles, respectively. Energies are given relative to the VBM. Schematic drawings of low-energy bands in (c) bulk $\mathrm{MoS}_{2}$ and (d) monolayer $\mathrm{MoS}_{2}$ showing the band gaps $\mathrm{E}_{g}$, as well as the valence band spin-orbit splitting $\Delta_{s o}$ and the $\Gamma$ valley band offset $\Delta_{\Gamma_{-K}}$ for the case of monolayer $\mathrm{MoS}_{2}$. The band structure was adopted from [103].

Figure 2.6 Absorption (gray lines) and PL (color lines) spectra of monolayer semiconducting TMDs crystals $\left(\mathrm{MoS}_{2}, \mathrm{MoSe}_{2}\right.$, $\mathrm{WS}_{2}, \mathrm{WSe}_{2}$ ). Measurements were made on layers produced by mechanical exfoliation of bulk crystals. The photoluminescence peak coincides with the bandgap exciton absorption peak. Adopted from [107].

Figure 2.7 (a) Raman active modes of monolayer TMDs. (b) Schematics of triangular shape TMDs, the grey atom is a transition metal and yellow is the chalcogen. (c) Raman spectra of $\mathrm{MoS}_{2}$ and $\mathrm{WS}_{2}$ showing vibrational modes inset are their triangular structure.

Figure 2.8 SHG characteristics from $\mathrm{MoS}_{2}$ (a) Power dependence of SHG from a monolayer $\mathrm{MoS}_{2}$, the upper inset shows the fundamental spectra (red) and the SHG (blue). The lower inset shows the layer-dependent intensity of the SHG. Adopted from [52]. (b) SHG and THG intensities as functions of the number of layers. Adopted from [122].

Figure 3.1 (a) Schematic diagram for the CVD synthesis of TMDs inside a quartz tube, showing the crucibles, and the substrate, two zones are heated independently at different temperatures. (b) CVD system at Protective Coatings and Nanostructured Materials Laboratory vandegraaff PUC-Rio. 
Figure 3.2 (a) Alumina crucible showing three different positions of the substrate. In every position substrate is facing downwards. (b) Temperature profile of the growth procedure, Ar flow rate is $100 \mathrm{sccm}$ during the whole process.

Figure 3.3 Optical images of some CVD grown TMDs. (a) $\mathrm{WS}_{2}$ monolayer on $\mathrm{SiO}_{2} / \mathrm{Si} 300 \mathrm{~nm}$. (b) $\mathrm{WS}_{2}$ monolayer on quartz. (c) Bilayer $\mathrm{MoS}_{2}$ on $\mathrm{SiO}_{2} / \mathrm{Si} 300 \mathrm{~nm}$. Scale bars are $10 \mu \mathrm{m}$

Figure 3.4 (a) Schematic of an optical microscope image formation. (b) ZEISS Axio Scope. A1 at Protective Coatings and Nanostructured Materials Laboratory Vandegraff PUC-Rio.

Figure 3.5 (a) Schematic of a SEM image formation. Adopted from [140]. (b) JEOL JSM-7610F Field Emission SEM at vandegraaff PUC-Rio.

Figure 3.6 Stokes and anti-Stokes Raman scattering.

Figure 3.7 (a) Schematics of Raman spectrometer. (b) NT-MDT, NTEGRA Spectrometer at Protective Coatings and Nanostructured Materials Laboratory Vandegraaff PUC-Rio.

Figure 3.8 Schematic diagram of the PL experimental setup. 52

Figure 3.9 Cantilever deflection detection scheme by means of a beam of laser. Adopted from [146].

Figure 3.10 Experimental setup for XPS. Adopted from [152].

Figure 3.11 General second harmonic generation phenomena.

Figure 3.12 (a) Schematic diagram of harmonic generation (SHG and THG) microscopy system. (b) Experimental setup for harmonic generation measurements at Mackgraphe, Mackenzie Presbiterian University, SP.

Figure 3.13 (a) Spin coater (model WS-650Hzb-23NPPB-UD-3, at labnano $\mathrm{CBPF}$ ) was used to cover our sample with a resist. (b) Heating the sample coated with resist on a hot plate.

Figure 3.14 Laser writer lithography system model Heidelberg $\mu \mathrm{PG}$ 101 Laser writer at lab nano CBPF.

Figure 3.15 (a) Design on a LibreCAD program. (b) Optical images of a sample after development on which dose test was performed with different laser powers.

Figure 3.16 Edwards thermal evaporator equipment at vandegraff PUC-Rio.

Figure 3.17 General device fabrication procedure. $\quad 67$

Figure 3.18 Experimental setup for transport measurements. $\quad 67$

Figure 3.19 Schematic of the experimental setup.

Figure 4.1 (a) Optical microscopy images of $\mathrm{MoS}_{2}$ grown on $\mathrm{SiO}_{2} / \mathrm{Si}$ $285 \mathrm{~nm}$, scale bars are $50 \mu \mathrm{m}$ and $20 \mu \mathrm{m}$. (b) Optical images of $\mathrm{WS}_{2}$ grown on $\mathrm{SiO}_{2} / \mathrm{Si} 400 \mathrm{~nm}$.

Figure 4.2 (a) AFM topography, profile of $\mathrm{MoS}_{2}$ flake, inset the line profile shows the step of $1 \mathrm{~nm}$. (b) AFM image of $\mathrm{WS}_{2}$ flake. $\quad 71$

Figure 4.3 Raman spectra of $\mathrm{MoS}_{2}$ at different excitation wavelengths. 71

Figure 4.4 Raman spectra of $\mathrm{WS}_{2}$ at different excitation wavelengths. 72

Figure 4.5 (a) Photoluminescence spectra of monolayer $\mathrm{MoS}_{2}$. (b)

Photoluminescence spectra of monolayer $\mathrm{WS}_{2}$. 
Figure 4.6 (a,b) Optical images of $\mathrm{MoS}_{2} / \mathrm{WS}_{2}$ vertical heterostructure scale bar is $20 \mu \mathrm{m}$. (c) Raman spectra of $\mathrm{MoS}_{2} / \mathrm{WS}_{2}$ heterostructure taken at point 1 and 2 as shown in figure 4.6(b). (d) Photoluminescence of the same triangle at point 1 and 2 .

Figure 4.7 Dependence of synthesis parameters in the growth process. (a) Optical images of single-crystalline $\mathrm{MoS}_{2}$ changing the precursor's ratio $\mathrm{NaNO}_{3} / \mathrm{MoO}_{3}$ from 0.3 to 3.0. (b) Optical images show the island coalescence and form the polycrystal $\mathrm{MoS}_{2}$ by increasing the time from 14 to $20 \mathrm{~min}$, scale bar $10 \mu \mathrm{m}$.

Figure 4.8 SEM images of $\mathrm{MoS}_{2}$ single crystals.

Figure 4.9 XPS experiments. (a) XPS spectra of the grown sample before and after water bath for 2 hr. (b) Detailed XPS spectra of $\mathrm{Na}$ 1s peak before and after water bath. Detailed XPS spectra and fitting of Mo and S peaks. (c) Molybdenum 3d spectrumof $\mathrm{MoS}_{2}$ grown on $\mathrm{SiO}_{2} / \mathrm{Si}$ substrate. (d) S 2p spectrum of the same sample.

Figure 4.10 DFT calculations of the formation energy. (a-f) Atomic positions after minimization of the energy and forces in a $3 \times 3$ fixed size supercell of $\mathrm{MoS}_{2}$ in the presence of $\mathrm{Na}$ atoms. The light blue, dark grey and dark yellow balls represent the $\mathrm{Na}$, Mo, and S atoms, respectively. (g) Formation energy per atom computed with the $\mathrm{MoS}_{2}$ constrained by the presence of the $\mathrm{Na}$ after the minimization (black circles) and fully relaxed (red circles). The full black and red lines are guides to the eyes. The vertical axis on the right side is a conversion of energy to temperature.

Figure 4.11 Final structures for large concentrations of $\mathrm{Na}$ in $\mathrm{MoS}_{2}$. (a-c) top view of the $3 \times 3 \mathrm{MoS}_{2}$ supercell with 7, 8, and $9 \mathrm{Na}$ atoms, respectively. (d-f) side view of the same structures in (a-c), respectively. The average distance between $\mathrm{Na}$ atoms and the plane with Mo are approximately $4.0 \AA$ (first layer) and 5.8 $\AA$ (second layer). For the low concentration regimes (i.e. $<0.45$ ) the average distance varies approximately linearly between 3.7 $\AA$ and $4.0 \AA$ with the number of the Na atoms in the supercell.

Figure 4.12 Characterization of single crystal. (a) AFM image of a monolayer of $\mathrm{MoS}_{2}$, inset the line profile shows the step of 1 $\mathrm{nm}$. (b) Raman spectra of $\mathrm{MoS}_{2}$ monolayer from $473 \mathrm{~nm}$ laser line took from (a). (c) mapping of $A_{1}^{\prime}$ intensity mode and (d) $E^{\prime}$ intensity mode, scale bar $1 \mu \mathrm{m}$. (e) The peak position maps of $E^{\prime}$ and (f) $A_{1}^{\prime}$ modes showing uniformity throughout the triangles area.(g) Photoluminescence spectra took of $\mathrm{MoS}_{2}$. (h) PL intensity map of A exciton, scale bar $1 \mu \mathrm{m}$. (i) fwhm of Photoluminescence.

Figure 4.13 Characterization of polycrystal. (a) AFM image of polycrystalline $\mathrm{MoS}_{2}$. (b) Raman mapping of $\mathrm{E}^{\prime}$ intensity mode and (c) $\mathrm{A}_{1}^{\prime}$ intensity mode, scale bar $2 \mu \mathrm{m}$. (d) peak position maps of $\mathrm{E}^{\prime}$ and, (e) $\mathrm{A}_{1}^{\prime}$ mode (f) PL intensity map, scale bar $2 \mu \mathrm{m}$. 
Figure 4.14 Analysis of defects using Raman spectroscopy. (a) Raman spectra of single and polycrystal of $\mathrm{MoS}_{2}$ with the fitting. (b) Normalized intensity of LA mode in the function of $E^{\prime}$ frequency. (c) Histograms of LA mode of single and polycrystals.

Figure 4.15 (a) Optical image $\mathrm{MoS}_{2}$ on glass scale bar is $10 \mu \mathrm{m}$.

(b) AFM image of a monolayer triangle with height of $\sim 1$ $\mathrm{nm}$ scalr bar is $2 \mu \mathrm{m}$. (c) Raman and, (d) PL spectra of the corresponding triangle. (e) Intensity map of $\mathrm{E}^{\prime}$, (f) $\mathrm{A}_{1}^{\prime}$ modes of the same triangle. (g) PL intensity map.

Figure 5.1 Optical microscopy image of the as-grown (a) $\mathrm{MoS}_{2}$ trilayer stacked with same crystal orientation between each layer scale bar is $20 \mu \mathrm{m}$ (b) $\mathrm{MoS}_{2}$ bilayer stacked with opposite crystal orientation to the monolayer (c) $\mathrm{WS}_{2}$ trilayer first two triangles are stacked with same crystal orientation while the third triangle is stacked with $60^{\circ}$ w.r.t other layers scale bar is $10 \mu \mathrm{m}$ (d) $\mathrm{WS}_{2}$ bilayer stacked with opposite crystal orientation (e) $\mathrm{MoS}_{2} / \mathrm{WS}_{2}$ heterostructure stacked at $0^{\circ}$ and (f) $60^{\circ}$, scale bar is $10 \mu \mathrm{m}$.

Figure 5.2 (a) Raman spectra of $\mathrm{MoS}_{2}$ trilayer stacked with same crystal orientation between each layer shown in figure 5.1(a). (b) Log of photoluminescence intensity of the three layers, decrease in intensity refers to shifting towards indirect band gap.

Figure 5.3 (a) Raman spectra of $\mathrm{WS}_{2}$ trilayer shown in figure 5.1(c). (b) Log of photoluminescence intensity of the three layers.

Figure 5.4 Band gap of $4 \mathrm{~L}, 2 \mathrm{~L}$, and $1 \mathrm{~L}$ of $\mathrm{MoS}_{2}$ calculated by Splandani et al. [9]. The solid arrows indicate the lowest energy transitions. The direct excitonic transitions occur at high energies at $\mathbf{K}$ point. With reduced layer thickness, the indirect bandgap becomes larger, while the direct excitonic transition barely changes. For monolayer $\mathrm{MoS}_{2}$, it becomes a direct bandgap semiconductor. This dramatic change of electronic structure in monolayer $\mathrm{MoS}_{2}$ can explain the observed jump in monolayer photoluminescence efficiency.

Figure 5.5 (a) Raman and (b) PL mapping images of the $\mathrm{WS}_{2}$ trilayer shown in figure 5.1(c).

Figure 5.6 Single spectra of $\mathrm{MoS}_{2}$ flake shown in figure 5.1(a), showing second-harmonic and third-harmonic signals.

Figure 5.7 Intensity dependence of the SHG signal with the polarization angle. (a) Trilayer (green) with a twisted angle of $0^{\circ}$ between bilayer (red) and monolayer (black). (b) bilayer (red) twisted $60^{\circ}$ from monolayer (black).

Figure 5.8 Schematics of (a) Top view of the $\mathrm{MoS}_{2}$ crystallographic orientation with respect to the incident laser polarization $\mathrm{e}_{\omega}$. (b) Orientational dependence of SHG.

Figure 5.9 Polarization plot shown in figure 5.7(a), fitted with a sine wave function (black line) showing no phase difference between each layer. 
Figure 5.10 Schematics of relative stacking orientation of the top and bottom layers. (a) $3 \mathrm{R}(\mathrm{AB})$ phase, the metal atom is located at the center of the bottom hexagon. Thus has a no-inversion symmetry. (b) $2 \mathrm{H}\left(\mathrm{AA}^{\prime}\right)$ phase, the metal atom of the second layer sits precisely on top of the chalcogen atom of the bottom layer, has the inversion symmetry.

Figure 5.11 THG, constant intensity with $\theta$ (a) Trilayer $\mathrm{MoS}_{2}$ shown in figure 5.1(a) (b) Bilayer $\mathrm{MoS}_{2}$ shown in figure 5.1(b).

Figure 5.12 Intensity dependence of the SHG signal with the polarization angle. (a) Trilayer (green) with twisted angle of $0^{\circ}$ between bilayer(red) and monolayer (black). (b) bilayer (red) twisted $60^{\circ}$ from monolayer (black).

Figure 5.13 Intensity dependence of the SHG signal with the polarization angle. (a) $\mathrm{MoS}_{2} / \mathrm{WS}_{2}$ heterostructure $0^{\circ}$. and (b) $\mathrm{MoS}_{2} / \mathrm{WS}_{2}$ heterostructure $59^{\circ}$.

Figure 5.14 Optical image of $\mathrm{MoS}_{2} / \mathrm{WS}_{2}$ heterostructure $60^{\circ}$, and its intensity dependence of the SHG signal with the polarization angle.

Figure 5.15 Schematics of relative stacking orientation of the top and bottom layers. (a) $\mathrm{MoS}_{2} / \mathrm{WS}_{2} 0^{\circ}$. (b) $\mathrm{MoS}_{2} / \mathrm{WS}_{2} 60^{\circ}$, mismatch between the masses of Mo and $\mathrm{W}$ atoms results in SHG.

Figure 5.16 Schematic for illustrating the vector superposition of the second harmonic fields.

Figure 6.1 Illustration of the structure of a FET

Figure 6.2 (a) Schematic representation of FET devices. (b) Optical image of a back gate FET device fabricated on $\mathrm{SiO}_{2} / \mathrm{Si}$, showing drain and source electrodes and channels $\mathrm{L}$ and $\mathrm{W}$.

Figure 6.3 Transport measurements. (a) Transfer curve $\left(\mathrm{I}_{D S}\right.$ vs. $\left.\mathrm{V}_{G}\right)$ with $\mathrm{V}_{D S}$ fixed at $1 \mathrm{~V}$, for $\mathrm{MoS}_{2}$ single crystal. (b) Output curve $\left(\mathrm{I}_{D S}\right.$ vs. $\left.\mathrm{V}_{D S}\right)$ at different $\mathrm{V}_{G}$ values, for single crystal. (c) Transfer curve $\left(\mathrm{I}_{D S}\right.$ vs. $\left.\mathrm{V}_{G}\right)$ with $\mathrm{V}_{D S}$ fixed at $1 \mathrm{~V}$ for $\mathrm{MoS}_{2}$ polycrystalline film. (d) Output curve $\left(\mathrm{I}_{D S}\right.$ vs. $\left.\mathrm{V}_{D S}\right)$ at different $\mathrm{V}_{G}$ values, for polycrystalline film.

Figure 6.4 (a) Optical image of a FET device fabricated after transferring $\mathrm{WS}_{2}$ on $\mathrm{SiO}_{2} / \mathrm{Si}$ substrate, scale bar is $10 \mu \mathrm{m}$. (b) Transfer curve $\left(\mathrm{I}_{D S}\right.$ vs. $\left.\mathrm{V}_{G}\right)$ with $\mathrm{V}_{D S}$ fixed at $1.5 \mathrm{~V}$ for $\mathrm{WS}_{2}$. (c) Output curve of $\mathrm{WS}_{2}\left(\mathrm{I}_{D S}\right.$ vs. $\left.\mathrm{V}_{D S}\right)$ for different $\mathrm{V}_{G}$ values.

Figure 6.5 (a) Optical image of $\mathrm{MoS}_{2} / \mathrm{WS}_{2}$ heterostructure device on $\mathrm{SiO}_{2} / \mathrm{Si} 285 \mathrm{~nm}$ substrate. The big triangle is the $\mathrm{MoS}_{2}$ and on top of it flower shape is an incomplete grown $\mathrm{WS}_{2}$ triangle. (b) Output curve of $\mathrm{MoS}_{2} / \mathrm{WS}_{2}\left(\mathrm{I}_{D S}\right.$ vs. $\left.\mathrm{V}_{D S}\right)$ for different $\mathrm{V}_{G}$ values.

Figure 6.6 Raman spectra of $\mathrm{MoS}_{2} / \mathrm{WS}_{2}$ device at different back gate voltages $\mathrm{V}_{G}$, laser wavelength $473 \mathrm{~nm}$. Red circles are experimental data points, the black lines are Lorentzian fits to the total spectrum.

Figure 6.7 (a) Peak position of $\mathrm{W}-\mathrm{A}_{1}^{\prime}$ and Mo- $\mathrm{A}_{1}^{\prime}$ as a function of $\mathrm{V}_{G}$. (a) FWHM of $\mathrm{W}-\mathrm{A}_{1}^{\prime}$ and Mo- $\mathrm{A}_{1}^{\prime}$ peak as a function of $\mathrm{V}_{G}$. 
Figure 6.8 (a) Intensity normalized with Si peak Mo- $\mathrm{A}_{1}^{\prime}$ and $\mathrm{W}-\mathrm{A}_{1}^{\prime}$ as a function of $\mathrm{V}_{G}$. (b) Normalized intensity of $\mathrm{D}$ peak as a function of $\mathrm{V}_{G}$. 


\section{List of tables}

Table 3.1 Growth parameters for different TMDs. In all synthesis powder is placed into the boat with the substrate facing down.

Table 5.1 Normalized SHG intensity and phase of TMD's.

Table 6.1 Average conductivity and mobility of TMDs. 
Knowledge is better than wealth: Knowledge protects you, whereas you have to protect wealth. Wealth decreases with spending, whereas knowledge increases with it.

Hazrat Ali (A.S), 


\section{List of Abreviations}

TMDs - Transition Metal Dichalcogenides

$\mathrm{MoS}_{2}$ - Molybdenum disulfide

$\mathrm{WS}_{2}$ - Tungsten disulfide

CVD - Chemical Vapor Deposition

2D - Two Dimension

BZ - Brillouin zone

hBN - Hexagonal boron nitride

VBM - Valence band maximum

CBM - Conduction band minimum

FWHM - Full width at half maximum

PL - Photoluminescence

AFM - Atomic Force Microscopy

XPS - X-ray Photoelectron Spectroscopy

SEM - Scanning Electron Microscopy

PL - Photoluminescence

LA - Longitudinal acoustic

TA - Transverse acoustic

IR - Infrared

DFT - Density functional theory

SHG - Second Harmonic Generation

THG - Third Harmonic Generation

$\chi^{(1)}-$ First order nonlinearity

$\chi^{(2)}-$ Second order nonlinearity

$\chi^{(3)}-$ Third order nonlinearity

FET - Field Effect Transistor

MOSFETs - Metal-oxide Field Effect Transistor 


\section{Introduction}

The discovery of graphene has opened an entirely new field of research in Material Science [1-3]. It became one of the most studied materials in the past years. Even though graphene has a unique electronic structure, exceptional strength, excellent electrical conductivity but it lacks a bandgap and consequently is not suitable for digital electronic applications. There are other layered materials that can be thinned down to monolayers [4]. One important family of layered or two-dimensional (2D) materials is the family of transition metal dichalcogenides (TMDs) [5-7]. Till now, more than 40 transition metal dichalcogenides have been discovered [8]. They have been studied for a long time in their bulk form $[9,10]$, but their properties when in the monolayer form differ from their bulk characteristics. In particular, molybdenum and tungsten based TMDs are atomically thin semiconductors with band gaps ranging from the visible to the near-infrared region. Bulk $\mathrm{MoS}_{2}$ and $\mathrm{WS}_{2}$ possess an indirect bandgap, while their monolayers have a direct bandgap [11]. So these two are more useful in all of the TMDs. This thesis does not attempt to cover all TMDs, our main interest lies in molybdenum and tungsten based semiconducting TMDs.

There are many methods for the synthesis of monolayer TMDs the simplest method is mechanical exfoliation from bulk $[12,13]$. However, this method is random and not scalable. It has some limitations regarding shape, size, and problems during transfer procedure which can introduce some impurities and cracks. Also, the adhesion between the substrate and TMD layer is not good in exfoliated samples. These facts may impair the performance of TMDs based devices [14]. In contrast, the direct growth method has the advantage of providing better integration between the substrate and TMDs. This integration is essential for nanoelectronic devices.

Beyond mechanical exfoliation, chemical vapor deposition (CVD) is the second most common technique used to prepare TMDs layered materials. Usually, TMDs are synthesized at high temperatures above $800^{\circ} \mathrm{C}$ [15-17] on $\mathrm{SiO}_{2}$, sapphire, and quartz substrates [18-20]. By changing the synthesis parameters e.g, temperature, precursor, atmospheric pressure, etc, we can grow different TMDs. There is no standard synthesis model which could be 
followed, different growth methods are reported following different parameters by different groups around the world [21]. Hence a reliable and repeatable direct synthesis method is needed for each TMD synthesis. In this thesis, many key elements in the synthesis procedure e.g, the position of the substrate, shape of the ceramic boat, etc, generally not mentioned in the articles, but very important in growth procedure will be discussed in details. Moreover, the high temperatures synthesis of TMDs is a barrier to mass production. One route to reduce the fabrication cost is to decrease the synthesis temperature. Lowtemperature synthesis of graphene, for example, became a useful addition in nanoelectronics [22-25]. These reports on graphene synthesis have shown great optical performances, avoids transfer defects, and has properties comparable to those of high temperature grown graphene [26,27]. This encourages the researchers to move on to low temperature synthesis of TMDs. Very little is known about the synthesis of TMDs on glass because it cannot reach high-temperature. The transition temperature of flat glass is $564^{\circ} \mathrm{C}$, so any recipe that works below this temperature will be useful in mass production and studying the transmission properties of TMDs. Also, tellurium assisted growth has able to decrease the synthesis temperature of TMDs [28]. Alkali metal salts are also known to be promoters in the growth of large area TMDs [29-31], but the knowledge about the low temperature synthesis using salts is limited. Recently, monolayers of $\mathrm{MoS}_{2}$ were grown at high temperatures on soda-lime glass. The sodium from the substrate can act as a catalyst and increase the growth rate [32-34]. Based on the characteristic that sodium increases the chemical reaction's velocity, we propose a new synthesis route at low-temperature mediated by sodium at atmospheric pressure. This sodium mediated low-temperature synthesis technique has enabled us to achieve $\mathrm{MoS}_{2}$ single crystals and polycrystals, on $\mathrm{SiO}_{2}$ and glass substrates.

Atomically thin semiconducting TMDs (e.g $\mathrm{MoS}_{2}, \mathrm{MoSe}_{2}, \mathrm{WS}_{2}$ ) have indirect bandgap in bulk form, while direct bandgap when thinned down to monolayer [35]. The transition from indirect to direct bandgap in monolayer TMDs give rise to strong photoluminescence $[36,37]$. Mak et al. have shown the variation of photoluminescence with the number of layers in $\mathrm{MoS}_{2}$ sample [38]. Semiconducting TMDs show remarkable interaction with light, over a broad bandwidth ranging from infrared to ultraviolet wavelengths. Monolayer $\mathrm{MoS}_{2}$ has an inter-band transition at the $\mathbf{K}$ and $\mathbf{K}^{\prime}$ points in the first Brillouin zone (BZ), where strong spin-orbit interaction splits the valence band into two subbands [39]. This gives rise to two peaks in the optical absorption spectra of $\mathrm{MoS}_{2}$, identified as A (1.85 eV) and B (2 eV) excitons [11]. Considerable efforts have been made to study the absorption and transmission properties of these 
materials [40-42].

Raman spectroscopy is the most powerful tool to characterize TMDs and other 2D materials, it can reveal information about the crystal and electronic structure, lattice vibrations and layer thickness. In general back-scattering Raman spectroscopy of semiconducting TMDs, exhibits two first-order Raman peaks, corresponding to the $\mathrm{A}_{1 g}$ out-of-plane and the $\mathrm{E}_{2 g}$ in-plane vibrational modes. Raman studies of single and few layers of $\mathrm{MoS}_{2}[43,44]$ and $\mathrm{WS}_{2}$ [45] have shown that the frequency of $\mathrm{E}_{2 g}$ mode decreases as the number of layers increases, whereas the frequency of $\mathrm{A}_{1 g}$ mode increases. Such behaviour is due to increase in dielectric screening which reduces the long-range Coulomb interaction between the effective charges with increasing number of layers. Raman spectroscopy was also used to identify several defect-induced Raman scattering peaks in $\mathrm{MoS}_{2}$ monolayer arising from zone-edge phonon modes [46]. The intensity of the defect peak is proportional to the density of defects, providing a practical route to quantify defects in monolayer $\mathrm{MoS}_{2}$ using Raman spectroscopy. However, very little is known about the defects related Raman phenomena in other monolayer TMDs.

Beyond linear optics, nonlinear optical properties of TMDs, play a key role in understanding the nanoscale light-matter interactions, as well as the structure of layered TMDs [47]. Second harmonic generation (SHG) is the most studied nonlinear process in TMDs [48]. SHG of the bulk TMDs is under study for long [49]. However, soon after the discovery of 2D-TMDs the SHG in monolayer has increased drastically. The second order nonlinearity $\chi^{(2)}$ in monolayer TMDs was reported to be $10^{3}$ times higher than the commercial nonlinear crystals (e.g, BBO, KTP, and $\mathrm{LiNbO}_{3}$ ) [50-52]. This makes them more favourable for practical use in future non-linear optical devices. Monolayers of TMDs are non-centrosymmetric and, therefore, second harmonic generation (as well as a third-harmonic generation) has been observed in many TMDs [53-59]. The angular dependence of SHG in monolayer TMDs shows strong symmetry dependence on its crystal lattice structure. Hence, SHG microscopy can serve as a powerful tool to non-invasively determine the crystalline directions of TMDs monolayers. Recently, polarization-resolved SHG microscopy was used to calculate the twist angle in two stacked layers of $\mathrm{WS}_{2}$, by measuring the crystal orientation of each layer [60].

In the $2 \mathrm{H}$ phase, bilayers of TMDs present the so-called $\mathrm{AA}^{\prime}$ interlayer stacking order, which is centrosymmetric and [61], therefore, does not allow for a second harmonic generation. In contrast, if we rotated two layers relative to each other, the centrosymmetry can be broken. Indeed, SHG in artificially stacked bilayers and heterostructures was reported before [62]. However, the 
study of SHG in as-grown bilayers and heterostructures with different rotate angle is unknown. Here, we report the SHG from the CVD grown of two and three layers TMDs with different phase stacking between the top and bottom layers, i.e., $0^{\circ}$ and $60^{\circ}$ stacking. The second harmonic generating reveals that CVD grown bilayers could possess two types of phase stacking (i.e $2 \mathrm{H}$ and $3 \mathrm{R}$ ).

Due to the direct bandgap present in monolayer TMDs, they are good candidates for digital electronic applications, which triggered the researchers to use these materials for optoelectronic devices. $\mathrm{MoS}_{2}$ and $\mathrm{WS}_{2}$ have proved to be a good field effect transistor with high electron mobility and charge transfer [63-72]. The highest carrier mobility yet reported is by Wei Chen et al. [33] is around $90 \mathrm{~cm}^{2} V^{-1} \mathrm{~s}^{-1}$ with $\mathrm{ON} / \mathrm{OFF}$ ratio of $10^{7} . \mathrm{MoS}_{2}$ has proved to be a good photodetector from visible to infrared range with high photoresponsivity [73-80]. The electrical performance of the device fabricated on the monolayer TMDs is directly related to the quality of the material. Hence, one can examine the quality of their grown TMDs by electrical measurements. The dependence of first-order Raman modes of $\mathrm{MoS}_{2}$ on electron doping was also reported by B. Chakraborty et al, in the top gate field effect transistor (FET) device configuration [81]. They have shown the redshift of $\mathrm{A}_{1 g}$ mode by applying the gate voltage in FET configuration and, simultaneously measuring the Raman spectra. Here, we have measured the dependence of Raman modes of CVD grown $\mathrm{MoS}_{2} / \mathrm{WS}_{2}$ heterostructure device with gate voltage.

This thesis is a pioneer work, on the synthesis and characterization of monolayer TMDs for our group. The main objective of this thesis was to develop reliable and repeatable methods for growing high-quality monolayer $\mathrm{MoS}_{2}, \mathrm{WS}_{2}$, and, their heterostructure by atmospheric pressure CVD system. We have grown $\mathrm{MoS}_{2}$ single crystals and polycrystalline film at low temperatures. The low temperature synthesis is mediated by sodium which is the key element in relaxing the growth temperature. Secondly, exploring the optical properties of CVD grown TMDs by optical microscopy, atomic force microscopy, X-Ray photoelectron spectroscopy, Raman and Photoluminescence spectroscopies. We also report the CVD synthesis of two and three layers of $\mathrm{MoS}_{2}$ and $\mathrm{WS}_{2}$ with different phase stacking between the top and bottom layers, i.e., $0^{\circ}$ and $60^{\circ}$ stacking. The structure and stacking sequences of bi-layer TMDs was revealed by the SHG. The SHG shows $0^{\circ}$ stacked bilayer has no inversion symmetry which refers to $3 \mathrm{R}(\mathrm{AB})$ and $60^{\circ}$ has inversion symmetry (Centrosymmetric) and has $2 \mathrm{H}\left(\mathrm{AA}^{\prime}\right)$ phase stacking. Finally, back gate FET devices were fabricated to investigate the electrical performances of our TMDs. 
And the effect of electron doping $\left(\mathrm{V}_{G}\right)$ on the vibrational modes was studied by Raman spectroscopy.

The thesis structure is divided into six chapters. Chapter 2 presents a brief explanation of TMDs, their crystalline and electronic structure. The stacking of two layers, Raman modes, direct and indirect bandgap in TMDs. The detailed explanations of materials, equipment, and CVD techniques used for the synthesis of TMDs. Chapter 3 explains various techniques employed to characterize the CVD grown samples, including Raman spectroscopy, atomic force microscopy, second harmonic generation, device fabrication, and finally electrical measurements. The experimental setups of all the characterization techniques are explained in details. Chapter 4 presents the characterizations of the CVD grown monolayer $\mathrm{MoS}_{2}, \mathrm{WS}_{2}$, and $\mathrm{MoS}_{2} / \mathrm{WS}_{2}$ by AFM, Raman, and PL spectroscopies are discussed. A detailed explanation of sodium mediated low temperature synthesis technique of $\mathrm{MoS}_{2}$ monolayer triangles and films. The catalytic behavior of sodium in decreasing the synthesis temperature is explained by first principle calculations. Chapter $\mathbf{5}$ presents the characterization of two and three layers of TMDs having different crystal orientations. Non-linear optical properties (second and third harmonic generation) of two and three layers $\mathrm{MoS}_{2}, \mathrm{WS}_{2}$, and $\mathrm{MoS}_{2} / \mathrm{WS}_{2}$ samples. Chapter 6 presents the electrical measurements, transport and output curves of FETs devices fabricated on $\mathrm{MoS}_{2}, \mathrm{WS}_{2}$ and $\mathrm{MoS}_{2} / \mathrm{WS}_{2}$ samples and the electro-Raman of $\mathrm{MoS}_{2} / \mathrm{WS}_{2}$ heterostructure device. 


\section{2}

\section{Properties of Transition Metal Dichalcogenides}

This chapter includes a brief explanation of TMDs, their crystalline structure, and the stacking of two layers. Optical phonons and electronic structure of TMDs. Linear and Non-linear optical properties of TMDs. Basic concepts about Raman modes, direct and indirect bandgap.

\section{1}

\section{Introduction to Transition Metal Dichalcogenides}

Transition metal dichalcogenides have the chemical formula $\mathrm{MX}_{2}$, where $\mathrm{M}$ is a transition metal e.g, (Mo, W), and $\mathrm{X}$ is a chalcogen atom ( $\mathrm{S}$, Se, or Te). The transition metals have d electrons in the outermost shells which play an important role in determining their properties, as they form a covalent bond with the chalcogen atoms [82]. When the covalent bond is formed in TMDs, the transition metal shares its electron to the chalcogen atom, filling the valence shell that is responsible for the planar structure of the TMDs. The atoms in two dimensional TMDs are spacially arranged in an $\mathrm{X}-\mathrm{M}-\mathrm{X}$ structure, each transition metal atom is sandwiched between two chalcogen atoms [35]. Figure 2.1 shows a periodic table. When a transition metal atom [group IV (Ti, Zr, Hf), group V (V, Nb, Ta), or group VI (Mo, W)] forms a bond with chalcogen atoms [group XVI (S, Se, Te)] we call it transition metal dichalcogenide. Various permutations of these elements result in over 40 different compounds having different physical and chemical properties. Some of them are semiconductors, some are insulators, conductors, and superconductors also, although not all of them are layered solids [63,83,84]. The most important of them are Mo and $\mathrm{W}$ based dichalcogenides and its heterostructures because they are widely used in optoelectronic device applications [72].

In TMDs, a monolayer is defined as a plane of metal atoms structured hexagonally, sandwiched between two hexagonally ordered planes of chalcogen atoms, where the oxidation states of the metal and chalcogen elements are +4 and -2 , respectively [85]. Layer by layer stacking of these monolayers forms the bulk. Its structure is similar to graphite and each layer has a thickness of $\sim 6-8 \AA$, having strong covalent bonding within the plane and weak Van 


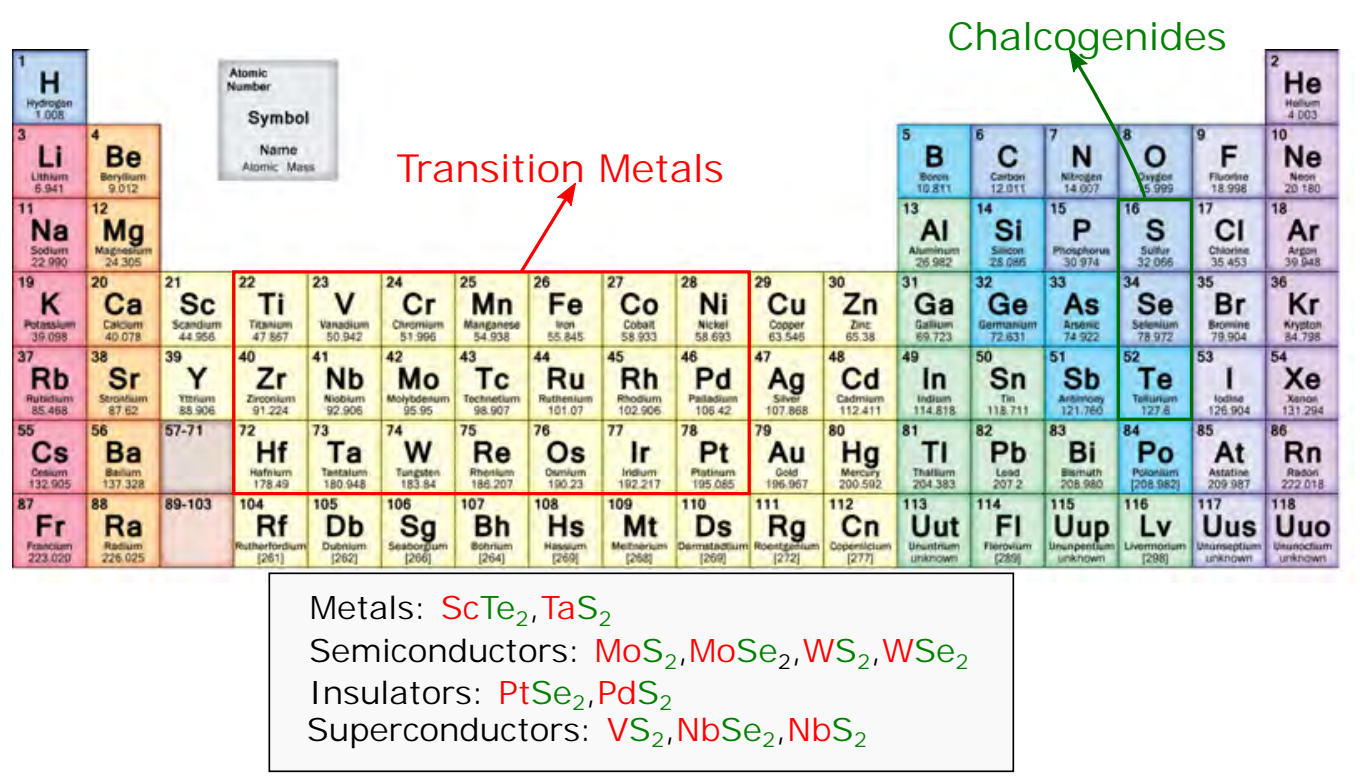

Figure 2.1: Periodic table and different TMDs.

der Waals interactions out of the plane. Bulk TMDs exhibit a wide variety of polymorphs and stacking prototypes because of the individual monolayer. The most commonly encountered polymorphs are 1T (trigonal prismatic), $2 \mathrm{H}$ (hexagonal), and 3R (rhombohedral), respectively, the digit indicates the number of $\mathrm{X}-\mathrm{M}-\mathrm{X}$ units in the unit cell, that is, the number of layers in the stacking sequence.

\subsection{1}

\section{Structure of TMDs}

Transition metal dichalcogenides $\left(\mathrm{MX}_{2}\right)$ differ significantly from classical 3-D counterparts. Individual layers of TMDs are weakly bonded to each other by Van der Waals forces, whereas within a single monolayer the metal and chalcogen atoms are covalently bonded. A monolayer in a TMD is an atomic trilayer structure, each layer of transition metal is sandwiched between two layers of chalcogen atoms $(\mathrm{X}-\mathrm{M}-\mathrm{X})$ see figure 2.2(a). Each transition-metal atom is surrounded by six chalcogen atoms that coordinate in an octahedron or a triangular prism as shown in figure 2.2(b) [86]. TMDs like $\mathrm{MX}_{2} ;(\mathrm{M}=\mathrm{Ti}, \mathrm{Zr}$, Hf ; X $=\mathrm{S}, \mathrm{Se}, \mathrm{Te}$ ) transition metal elements prefer the octahedral coordination because the strong ionic bonding results in the Coulomb repulsion between the inter-layer chalcogen atoms. On the other hand, semiconducting TMDs $\mathrm{MX}_{2}$; (i.e ; $\mathrm{M}=\mathrm{Mo}, \mathrm{W}$, and $\mathrm{X}=\mathrm{S}$, Se) are generally found a in triangular prism, whose bonding has more covalent nature [87].

The three atomic layers $(\mathrm{X}-\mathrm{M}-\mathrm{X})$ in semiconducting TMDs together 
(a)

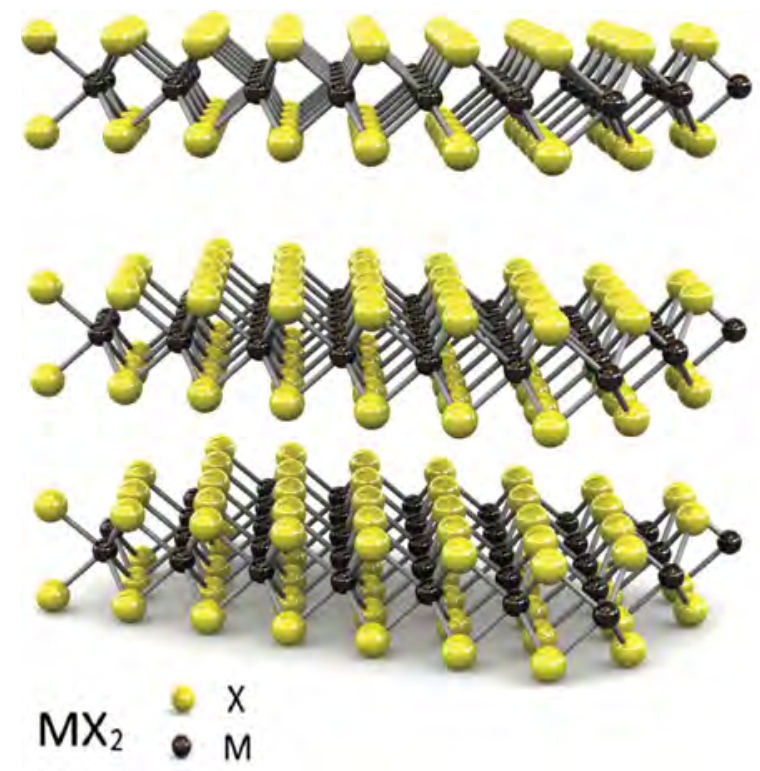

(b)

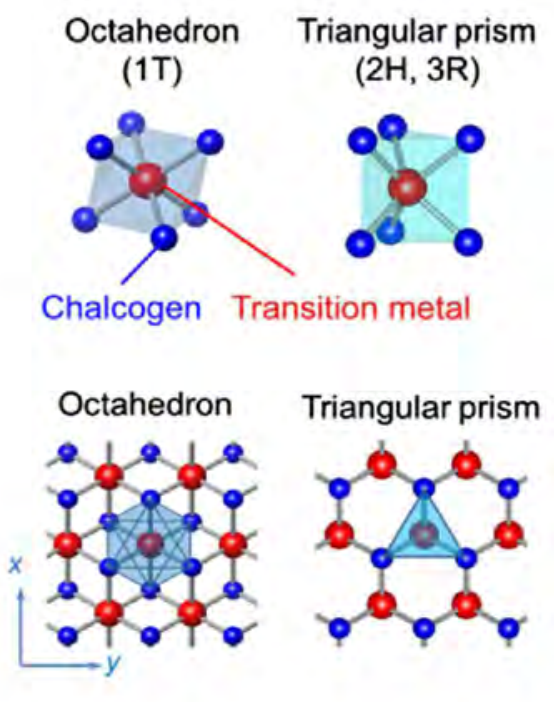

Figure 2.2: (a) 3D representation of the layered structure of transition metal dichalcogenides (TMDs). Adopted from [63]. (b) Archetypal coordination in TMDs. Octahedral coordination (left) triangular prismatic coordination (right) and top view of monolayers constructed from octahedral (left) and triangular prismatic (right) coordination. Adopted from [86].

form a $2 \mathrm{D}$ hexagonal lattice, with the A-sublattice being the metal atom per site and B-sublattice being the two chalcogen atoms per site. The hexagonal lattice has 3-fold symmetry or honeycomb structure like graphene and can allow inversion symmetry. For an even number of monolayers, the crystal structure has an inversion center, while for a perfect monolayer or any odd number of layers, the crystal does not have the inversion symmetry. Trigonal prism structure in $2 \mathrm{H}$ stacking type the unit cell contains two prisms of adjacent layers. The symmetry $2 \mathrm{H}-\mathrm{MX}_{2}$ belongs to the space group $\left(D_{6 h}\right)$ [88]. The space group for a single layer is $\left(D_{3 h}\right)$ due to the lack of translational symmetry along the z-axis. Similarly, for a few-layer system with even numbers of layers belong to the space group $\mathrm{D}_{6 h}$ (with inversion symmetry), and systems with odd numbers of layers to the $\mathrm{D}_{3 h}$ space group (without inversion symmetry) [89,90].

\subsection{2}

\section{Stacking Sequences of Two Layers}

Since the two layers of a TMDs are bound together via weak Van der Waals attractions, this allows the layers to be stacked with different orienta- 
tions and can break the inversion symmetry [62]. Two layers of semiconducting (Trigonal prism) TMDs can possess two types of phase stacking $2 \mathrm{H} / \mathrm{AA}^{\prime}$ and $3 \mathrm{R} / \mathrm{AB}$ phase stacking $[88,91,92]$. Monolayer has an identical structure in $2 \mathrm{H}$ and $3 \mathrm{R}$ phases, the difference lies in the stacking order of the monolayers in the layered structures. The $2 \mathrm{H}$ phase of the bulk crystal has the hexagonal symmetry, having two monolayers per repeat unit, whereas the $3 \mathrm{R}$ phase has the rhombohedral symmetry, having three layers per repeat unit. First-principles calculations show that the bilayer in $2 \mathrm{H}$ stacking is more stable than the $3 \mathrm{R}$ stacking [93]. In $2 \mathrm{H} / \mathrm{AA}^{\prime}$ phase stacking $\mathrm{M}$ atom of first layer sits precisely on top of $\mathrm{X}$ atom of the second layer and shows inversion symmetry, while in $3 \mathrm{R} / \mathrm{AB}$ phase stacking $\mathrm{M}$ atom is staggered a little from $\mathrm{X}$ atom of the other layer (no-inversion symmetry) see figure $2.3[88,94]$.

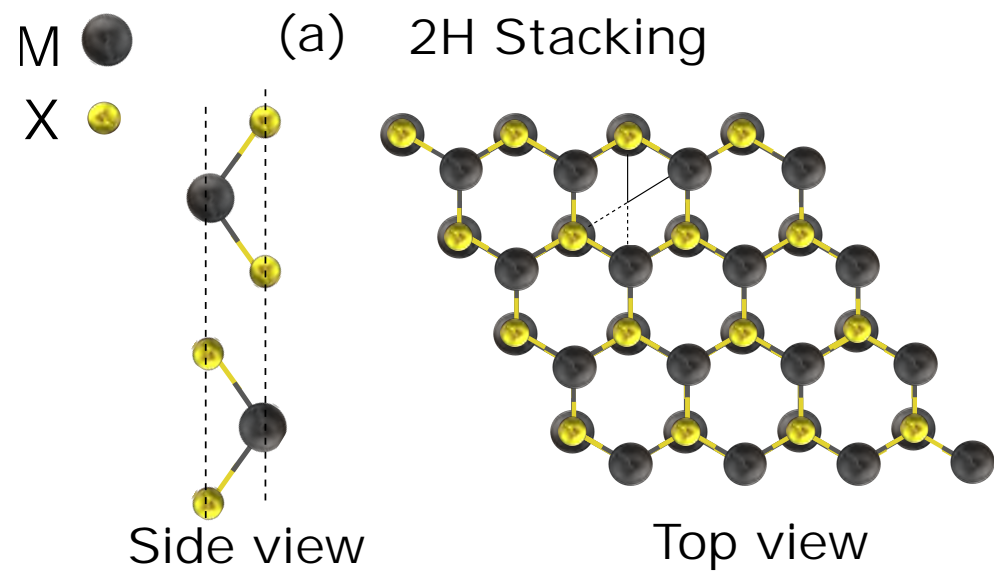

(b) 3R Stacking
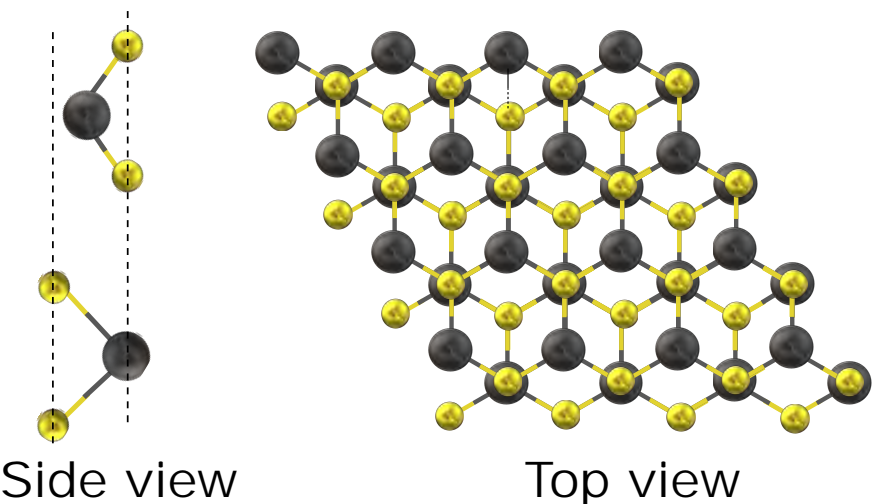

Top view

Figure 2.3: Side and top views of (a) $2 \mathrm{H}$ stacking pattern of bilayer $\mathrm{MX}_{2}, 2 \mathrm{H}$ is the most stable stacking pattern (also named as AA' stacking)(b) 3R (also named as AB stacking)

This stacking can also be used to control TMDs based device functionalities due to the effect of stacking on the electronic band structure, valley polarization, and nonlinear optical properties [62,93, 95]. 


\section{2}

\section{Phonon Dispersion}

From solid-state physics, we know that a usual semiconductor TMDs has a parabolic energy dispersion for electron and holes, and its joint density of state near the bandgap is a step function. The phonon dispersion relations of monolayer $\mathrm{MoS}_{2}$ and $\mathrm{WS}_{2}$ as calculated by A.Molina, et al. by density functional theory and is shown in figure 2.4 [90]. The vibrational modes are dispersive with respect to wave vector $\mathbf{q}$ The phonon dispersion has three acoustic and six optical branches derived from the nine vibrational modes at the $\Gamma$ point.

(a)

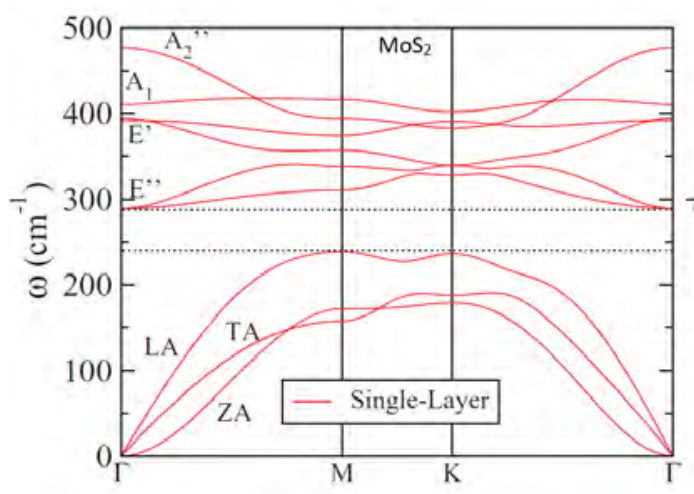

(b)

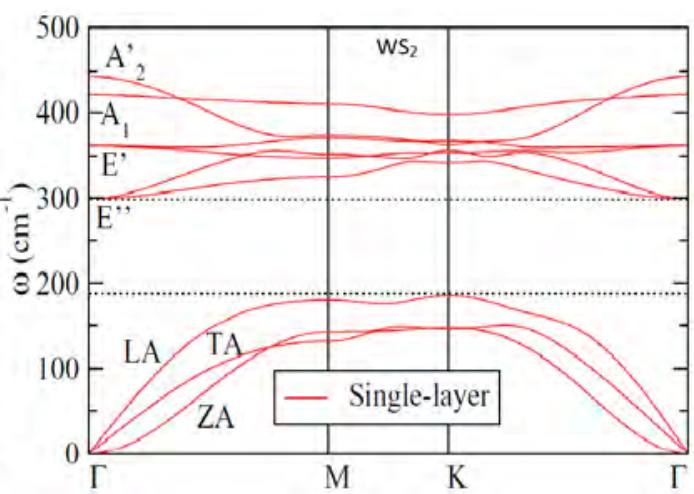

Figure 2.4: (a) Phonon dispersion curve of monolayer $\mathrm{MoS}_{2}$ calculated by A.Molina, et al. [90]. (b) and $\mathrm{WS}_{2}$.

The three acoustic branches are the in-plane longitudinal acoustic (LA) and transverse acoustic (TA), and the out-of-plane acoustic modes (ZA). The in-plane (LA and TA) have a linear dispersion and have higher energy than (ZA). The six optical branches are two out-of-plane transverse optical $\left(\mathrm{TO}_{1}, \mathrm{TO}_{2}\right)$, two in-plane longitudinal optical $\left(\mathrm{LO}_{1}, \mathrm{LO}_{2}\right)$, and two out-ofplane optical $\left(\mathrm{ZO}_{1}, \mathrm{ZO}_{2}\right)$ branches. These six optical modes at the $\Gamma$ point correspond to the irreducible representations $\mathrm{E}^{\prime \prime}\left(\mathrm{LO}_{1}\right.$ and $\left.\mathrm{TO}_{1}\right), \mathrm{E}^{\prime}\left(\mathrm{LO}_{2}\right.$ and $\left.\mathrm{TO}_{2}\right), \mathrm{A}_{2}^{\prime \prime}\left(\mathrm{ZO}_{1}\right)$ and $\mathrm{A}_{1}\left(\mathrm{ZO}_{2}\right)$ see figure 2.4. Because, $\mathrm{MX}_{2}$ compounds are slightly polar materials, certain IR-active phonon modes (e.g., $\mathrm{E}^{\prime}$ ) show LO-TO splitting because of the coupling of the lattice to the macroscopic electric field created by the relative displacement of $\mathrm{M}$ and $\mathrm{X}$ atoms in the long-wavelength limit $[90,96]$. There is also a bandgap between optical and acoustic branches, of $\sim 100 \mathrm{~cm}^{-1}$ for $\mathrm{MoS}_{2}$ and $\sim 30 \mathrm{~cm}^{-1}$ for $\mathrm{WS}_{2}$ [97-99]. 


\section{3 \\ Electronic Dispersion}

Looking from the top, the crystal structure of TMDs monolayer is a honeycomb lattice (figure 2.2(b)) similar to that of the graphene. In contrast to graphene, the A and B sublattices are occupied by different atoms. This has an immediate consequence in the electronic structure, lifting the degeneracy in the electronic structure at the $\mathbf{K}\left(\mathbf{K}^{\prime}\right)$ point in the first BZ. Degeneracy is responsible for the Dirac cone dispersion relation in graphene. In contrast, in monolayer $\mathrm{MoS}_{2}$, a substantial energy gap is present. As the projection in the plane exhibits a hexagonal crystal structure similar to graphene, the edge terminations of a $\mathrm{MoS}_{2}$ sheet can be zigzag, armchair, chiral, or a mixture of these types. The lift of the decency at $\mathbf{K}\left(\mathbf{K}^{\prime}\right)$ points in the first BZ creates a desirable bandgap in TMDs. The electronic band structure of TMDs depends on the transition metal and chalcogen atoms, thickness, and stacking sequence of each layer [100]. Electronic structure calculations have been playing an important role in understanding the physical properties of monolayer TMDs.

The electronic structure near the bandgap has the main contribution from $\mathrm{d}_{z^{2}}$ (out-of-plane) and $\mathrm{d}_{x^{2}-y^{2}}$ and $\mathrm{d}_{x y}$ (in-plane) orbitals of transition metal (M) atoms and $\mathrm{p}_{z}$ orbitals of chalcogenide (X) atoms [101]. The direct bandgap in $\mathrm{MX}_{2}$ (TMDs) depends on the localized d-orbital of the transition metal (M) atom, which is not much affected by the inter-layer coupling due to its location in the unit cell. The indirect bandgap in $\mathrm{MX}_{2}$ (TMDs) depends on the overlap between the d orbital of a transition metal (M) and the $\mathrm{p}_{z}$ orbital of chalcogenide atoms, which strongly depends on the interlayer coupling. Hence, as the number of layers is decreased, the intrinsic direct bandgap of the material becomes more prominent [102].

Figure 2.5(a,b) show the band structure of $\mathrm{MoS}_{2}$ monolayer and bulk and indicates the direct and indirect bandgaps, calculated by O. Yazyev et al. using density functional theory (DFT) within the generalized gradient approximation (GGA) [103]. Schematic illustration of the band structure of $\mathrm{MoS}_{2}$ is shown in figure $2.5(\mathrm{c}, \mathrm{d})$. Theoretical value of the bandgap of bulk $\mathrm{MoS}_{2}$ is $0.88 \mathrm{eV}$ obtained using DFT-GGA, with the valence band maximum (VBM) at the $\Gamma$ point in the Brillouin zone and the conduction band minimum (CBM) along the $\Gamma$-K direction. VBM and $\mathrm{CBM}$ are indicated by red and blue circles in figure 2.5. The experimentally observed bandgap of bulk $\mathrm{MoS}_{2}$ is $\sim 1.3 \mathrm{eV}$ [38]. As the number of layers decreases, the valence band maxima, and conduction band minima shift towards $\mathbf{K}$ due to quantum confinement, making it a direct bandgap semiconductor at the monolayer. Also, by decreasing the number of layers, the bandgap shifts upward in energy. That is attributed to the quantum 


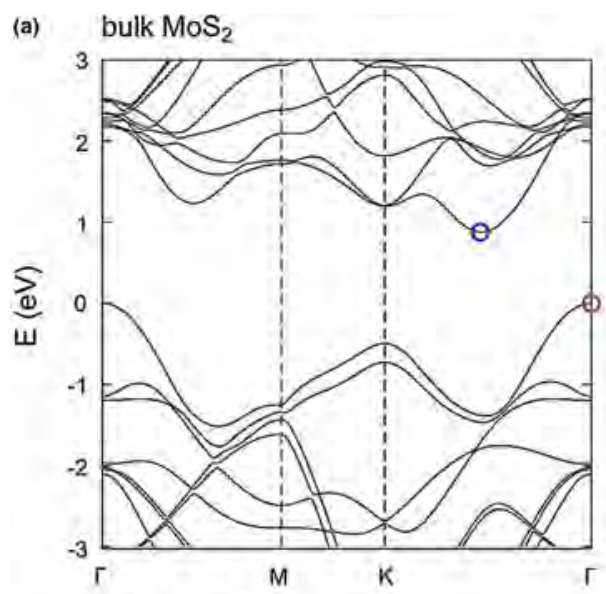

(c)
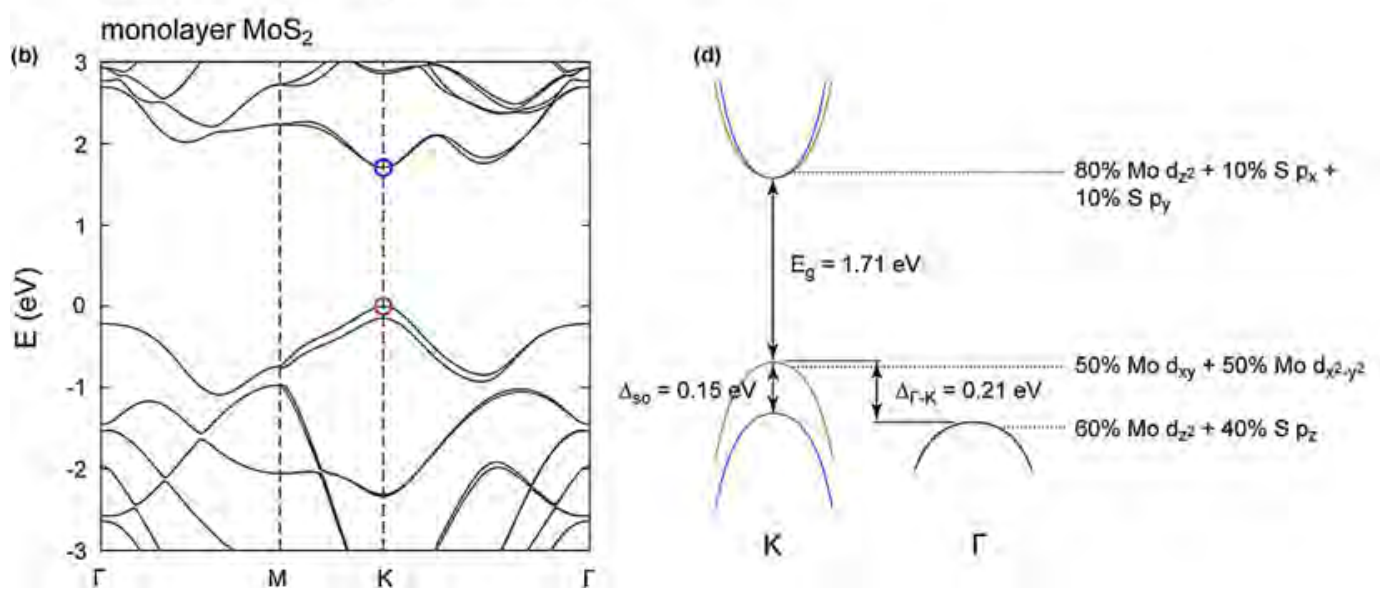

Figure 2.5: Electronic band structures of (a) bulk $\mathrm{MoS}_{2}$ and, (b) monolayer $\mathrm{MoS}_{2}$ calculated from first principles using DFT-GGA approach. Valence band maxima and conduction band minima are indicated by red and blue circles, respectively. Energies are given relative to the VBM. Schematic drawings of low-energy bands in (c) bulk $\mathrm{MoS}_{2}$ and (d) monolayer $\mathrm{MoS}_{2}$ showing the band gaps $\mathrm{E}_{g}$, as well as the valence band spin-orbit splitting $\Delta_{\text {so }}$ and the $\Gamma$ valley band offset $\Delta_{\Gamma_{-K}}$ for the case of monolayer $\mathrm{MoS}_{2}$. The band structure was adopted from [103].

confinement of charge carriers which is common in nanosystems $[104,105]$. The bandgap obtained from DFT-GGA calculations for monolayer $\mathrm{MoS}_{2}$ is $1.71 \mathrm{eV}$, while its experimentally observed value is $\sim 1.85 \mathrm{eV}$.

The transition from indirect to direct bandgap exhibits strong photoluminescence increase in order of $\left(\times 10^{4}\right)$ as compared to bulk that is attributed to the fact that the electron is no longer passing through the intermediate state and transition occurs directly. This indirect to direct bandgap transition was confirmed by photoluminescence experiments, where the authors observed a shift of the indirect PL peak with the number of layers and enhancement of the PL signal in monolayer compared to the few-layers and bulk $[11,38]$. 


\section{4}

\section{Linear Optical Spectroscopy}

The study of the interaction between electromagnetic radiation (light) and matter is known as optical spectroscopy. The standard setup for linear optical spectroscopy consists of a light source, the sample under consideration, a spectrometer to disperse the light, a detection unit and a computer to collect and plot the data. Linear optical spectroscopy includes reflection, transmission, and luminescence spectroscopy. Other techniques include photoluminescence spectroscopy and measurements of the luminescence yield or Raman spectroscopy. Simple linear methods such as linear absorption and photoluminescence spectroscopy were being practised for qualitative and quantitative analysis of TMD materials much prior to the existence of the laser.

Upon interacting with light, electrons in the TMDs can be excited from the valence band to the conduction band. In the relaxation process, the excited electrons will decay to the ground states with subsequent emission of light called photoluminescence. Monolayer TMDs (e.g $\mathrm{MoS}_{2}$ and $\mathrm{WS}_{2}$ ), are direct bandgap semiconductors with PL emission in the visible to near-infrared range. Although they are atomically thin (few nanometers), they give strong interaction with light.

Now, consider the light (photons) interacts with a TMD material if the lattice vibrations (phonons) are also involved in the optical transitions. The generated photon can have lower/higher energy than the incident photon, and the energy difference is related to a specific phonon mode. This inelastic light scattering phenomenon is known as Raman scattering. Raman scattering is very rare compared to elastic scattering (Rayleigh scattering), approximately one in a million photons have inelastic scattering and have a difference in energy. This is attributed to the energy absorption by the atoms or molecules and the energy difference is related to the chemical structure of the molecule responsible for the spread. Raman spectroscopy has become one of the most powerful tool for analyzing the electrons, phonons and their coupling in TMDs [106]. It provides us a chemical fingerprint of the substance.

\subsection{1}

\section{Photoluminescence in TMDs}

Figure 2.6, shows the absorption and photoluminescence spectra of different TMDs, adopted from [107]. The absorption and PL spectra of TMDs are co-related, for $\mathrm{MoSe}_{2}\left(\mathrm{WSe}_{2}\right)$, the absorption peak at a wavelength of $800 \mathrm{~nm}(750 \mathrm{~nm})$ corresponds well with the PL peak at this wavelength. In contrast, for $\mathrm{MoS}_{2}\left(\mathrm{WS}_{2}\right)$, the absorption and $\mathrm{PL}$ peaks are shifted. This 
shift in peak position is attributed to the inhomogeneity of the dielectric environment, which affects the surface interaction of monolayer $\mathrm{MoS}_{2}\left(\mathrm{WS}_{2}\right)$ and changes the binding energy of the excitons. An exciton is a hydrogenlike complex consists of a bounded electron-hole pair. Exciton is formed by the Coulomb attraction between two oppositely charged quasiparticles. It is an electrically neutral quasiparticle that exists in insulators, semiconductors, and some liquids. It is regarded as an elementary excitation of condensed matter that can transport energy without transporting the net electric charge. A monolayer of $\mathrm{MoS}_{2}$ can absorb $10 \%$ of incident light with energy above the bandgap [108].

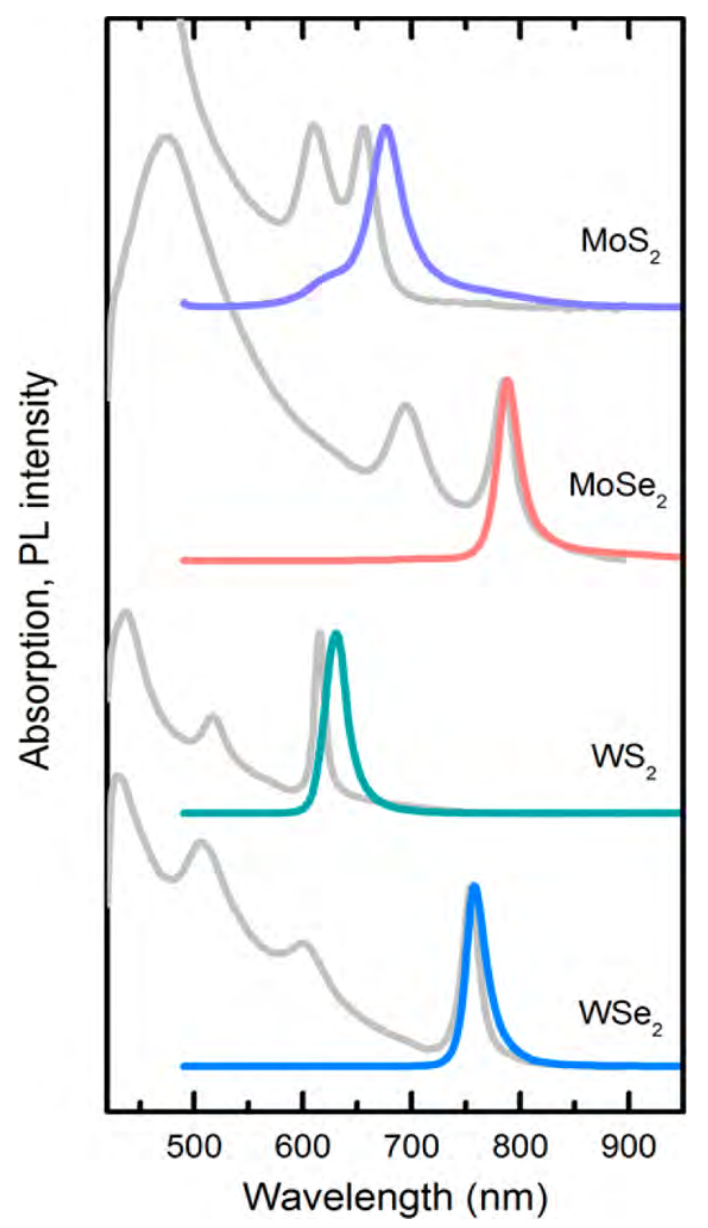

Figure 2.6: Absorption (gray lines) and PL (color lines) spectra of monolayer semiconducting TMDs crystals $\left(\mathrm{MoS}_{2}, \mathrm{MoSe}_{2}, \mathrm{WS}_{2}, \mathrm{WSe}_{2}\right)$. Measurements were made on layers produced by mechanical exfoliation of bulk crystals. The photoluminescence peak coincides with the bandgap exciton absorption peak. Adopted from [107].

In TMDs, the PL is influenced by many factors including the number of layers, strain, and interfacial interactions such as charge transfer, photonic nanostructures, etc. As already mentioned, these materials have bandgaps 
that vary depending on the number of layers [38]. The monolayers are direct bandgap semiconductors with the range of $\sim 650 \mathrm{~nm}$ to $1750 \mathrm{~nm}$. The direct transition of electrons without any change in moment makes them good candidates for light emitting devices. The PL spectrum of monolayer TMDs is characterized by two distinctive peaks, which are attributed to the excitonic transitions at the $\mathbf{K}$ (or $\mathbf{K}^{\prime}$ ) valley, and are labeled A (lowest energy) and B (highest energy). In the case of $\mathrm{MoS}_{2}$, the experimental values found for these energy transitions are $\sim 1.8-1.9 \mathrm{eV}$ (A exciton) and $\sim 2 \mathrm{eV}$ (B exciton), as can be seen in figure 2.6. These two peaks in (figure 2.6), are due to a strong excitonic effect in these TMD materials. The separation of A and B excitons come from the spin-orbit splitting of the top of the valence band at the $\mathbf{K}$ point from $\mathrm{d}_{x^{2}-y^{2}}$ and $\mathrm{d}_{x y}$ orbitals to $\mathrm{d}_{z^{2}}$ orbital [109].

The transient absorption spectroscopy has observed intervalley biexcitons in monolayer $\mathrm{MoS}_{2}$, which is the interactions between two excitons and result in the formation of bound quasiparticles [110]. In Van der Waals heterostructures and doped TMDs trions are also observed, which are formed by excitons excited in the one TMD layer and electrons transferred from the other layer [111].

The optical properties of these TMDs also vary according to the polarization. For example, Cao et al. study the absorption and PL of $\mathrm{MoS}_{2}$ in circularly polarized light [112]. They studied the $\mathrm{MoS}_{2}$ samples with different number of layers and showed that if PL is excited with circularly polarized light, the luminescence of the material also has substantial circular polarization. First principle calculations also showed that monolayer $\mathrm{MoS}_{2}$ is an ideal material for valleytronics [113]. The PL spectrum is also temperature dependent [114]. At low temperatures, the excitonic features can be easily resolved from one another and their intensities are usually higher than in room temperature measurements. The PL spectrum is also influenced by electron doping [115], as demonstrated by A.K. Newaz et al. both PL intensity and absorption can be controlled by gate voltage in monolayer $\mathrm{MoS}_{2}$. Such behavior is due to the interaction of excitons with the charge carriers [116].

\subsection{2}

\section{Raman Modes in TMDs}

The general back-scattering, Raman spectroscopy of $2 \mathrm{H}-\mathrm{MX}_{2}$ exhibits two first-order Raman peaks, corresponding to the $\mathrm{A}_{1 g}$ out-of-plane and the $\mathrm{E}_{2 g}$ in-plane vibrational modes. The first-order Raman band arises from a single phonon at the center of Brillouin zone while the second-order Raman band arises from multiple two-phonon processes across the whole Brillouin zone. In 
case of a monolayer of $1 \mathrm{H}-\mathrm{MX}_{2}$, it has three zone-centre and belongs to the space group $\mathrm{D}_{3 h}$. Irreducible representations of this point group shows $\mathrm{A}_{1}^{\prime}$ and $\mathrm{E}^{\prime}$ first-order Raman active modes, which corresponds to the out-of-plane $\mathrm{A}_{1 g}$ and in-plane $\mathrm{E}_{2 g}$ of $2 \mathrm{H}-\mathrm{MX}_{2}$ (space group $\mathrm{D}_{6 h}$ ) [117, 118]. In $\mathrm{A}_{1}^{\prime}$ phonon mode the transition metal $(\mathrm{M})$ atom does not move while chalcogenide $(\mathrm{X})$ atoms vibrates in the out of plane direction. In $\mathrm{E}^{\prime}$ phonon mode chalcogenide atoms vibrate in-phase while, the transition metal atom vibrate in-phase but in an opposite direction to chalcogenide atoms, see figure $2.7(\mathrm{a})$.

(a)

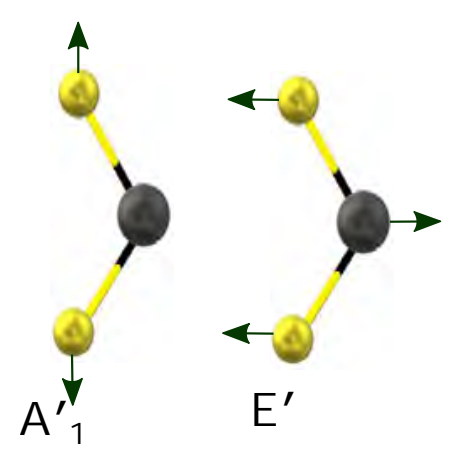

(b)

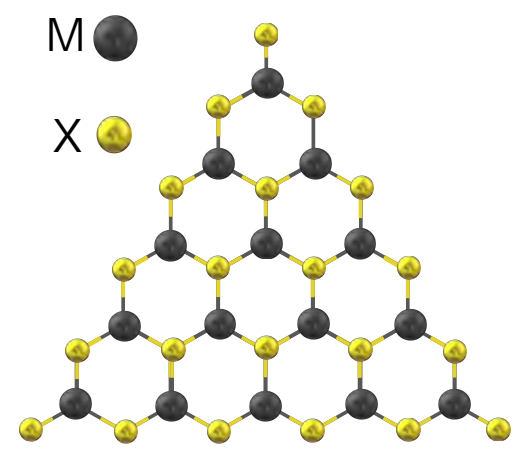

(c)

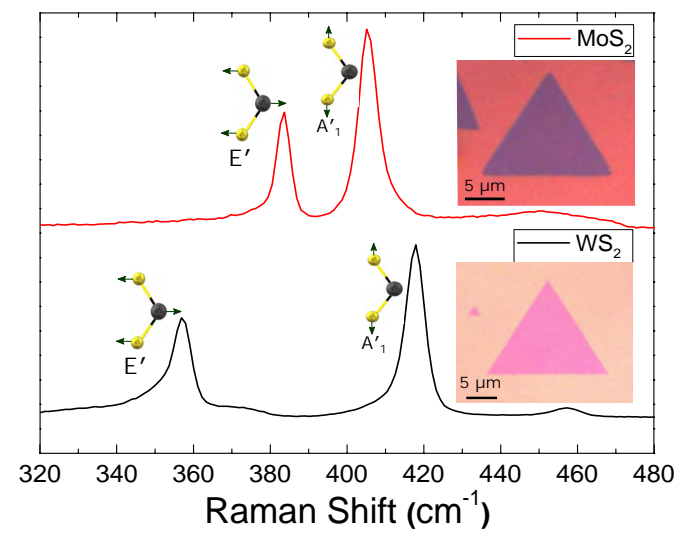

Figure 2.7: (a) Raman active modes of monolayer TMDs. (b) Schematics of triangular shape TMDs, the grey atom is a transition metal and yellow is the chalcogen. (c) Raman spectra of $\mathrm{MoS}_{2}$ and $\mathrm{WS}_{2}$ showing vibrational modes inset are their triangular structure.

Figure 2.7(c) shows the Raman spectra taken from $\mathrm{WS}_{2}$ and $\mathrm{MoS}_{2}$ monolayer using a $473 \mathrm{~nm}$ wavelength excitation laser. This shows only two Raman active modes corresponding to $\mathrm{E}^{\prime}$ and $\mathrm{A}_{1}^{\prime}$. The optical images (inset) of $\mathrm{WS}_{2}$ and $\mathrm{MoS}_{2}$ monolayer show the triangular structure. Generally, $\mathrm{MoS}_{2}$ and $\mathrm{WS}_{2}$ monolayer grow in triangular shape in CVD process. As all shapes of domains start growing from a hexagonal nucleus, but in sulphur rich atmosphere it always terminates with sulphur atom $[119,120]$. The schematic 
of triangular shape TMD is shown in figure 2.7(b), the triangular shape is only possible with the edge atom being sulphur.

\section{5}

\section{Non-Linear Optical Spectroscopy}

During light-matter interaction, the presence of intense fields can cause non-linear generation of new frequencies. The nonlinear effects occurs either due to intensity dependence of refractive index of the nonlinear medium or due to inelastic-scattering phenomenon. While nonlinear medium are the medium in which the dielectric polarization $(\mathrm{P})$ responds nonlinearly to the Electric field (E) of the light. The study of non-linear optical spectroscopy such as second harmonic generation and third harmonic generation (THG), has been widely reported in TMDs and other 2D materials, such as graphene, black phosphorus, hBN, etc [121]. TMDs with broken inversion symmetry can give rise to SHG (a non-linear optical effects). This phenomenon occurs due to the presence of a non-linear response in the material, it is largely used to characterize these materials. SHG is the most studied nonlinear effect in TMDs. In addition, the SHG can be used in photonic devices such as modulators, frequency converters, and in the production of new frequency lasers.

(a)

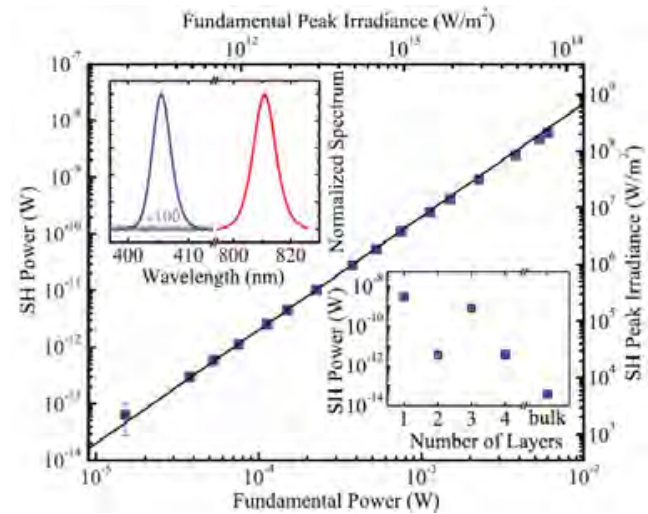

(b)

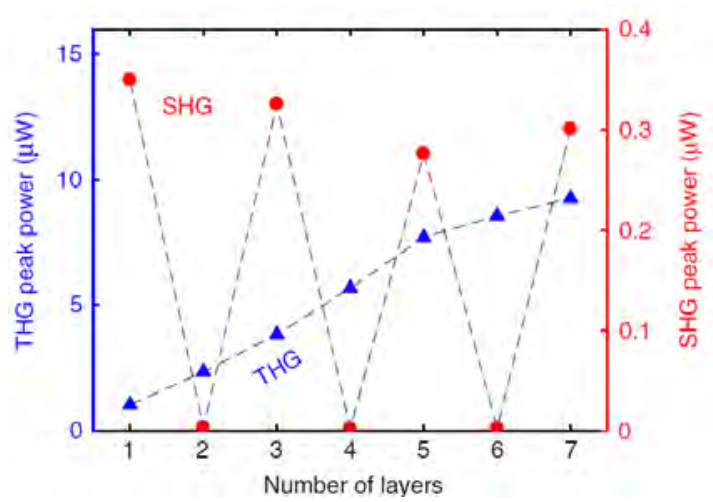

Figure 2.8: SHG characteristics from $\mathrm{MoS}_{2}$ (a) Power dependence of SHG from a monolayer $\mathrm{MoS}_{2}$, the upper inset shows the fundamental spectra (red) and the SHG (blue). The lower inset shows the layer-dependent intensity of the SHG. Adopted from [52]. (b) SHG and THG intensities as functions of the number of layers. Adopted from [122].

Figure 2.8(a) shows an example of SHG of an $810 \mathrm{~nm}$ Ti:sapphire laser pulse, from monolayer $\mathrm{MoS}_{2}$ and its dependence on the power and layer number [52]. A. Säynätjoki et al. studied the layer-dependent SHG in exfoliated $\mathrm{MoS}_{2}$ [122]. They observed SHG only in odd-layers and SHG vanished at even- 
layers see figure 2.8(b). This is due to the fact, that the inversion symmetry is restored for even-layers of $2 \mathrm{H}$-stacking TMDs.

Hsu et al. demonstrated the SHG in artificially stacked layers of TMDs with arbitrary angles [62]. They found that the SHG of artificially stacked bilayers is the coherent superposition of SHG fields of the individual monolayers, with a phase difference depending on the stacking angle. The interference mainly depends on the twisted angle but not the material constituent, which makes SHG as a sensitive method to examine the bilayer crystal orientation. $\mathrm{Yu}$ et al. demonstrated charge induced SHG in bilayer $\mathrm{WSe}_{2}$ [123]. They use the back gate FET configuration, by applying gate voltage the charge was accumulated in transition metal planes within the layer. The accumulation of the charge leads to a nonuniform electric distribution due to the screening effects. As a result, the inversion symmetry was broken and SHG was enhanced.

Third harmonic generation (THG) and four-wave mixing (FWM) are other nonlinear optical processes that originated from the third-order susceptibility $\chi^{(3)}$. THG has been widely investigated in many TMDs $[47,124]$. In-contrast to SHG, THG does not require the absence of the inversion symmetry and can be observed both in odd and even-layers. As can be seen in figure 2.8(b), THG signal intensity monotonically increases with the number of layers. Saturable absorption is another third-order nonlinear process and attributed to the imaginary part of $\chi^{(3)}$. TMDs are also being used as a saturable absorber. For example $\mathrm{MoS}_{2}$ shows high transmission and low non-saturable loss making it a potential candidate for application in mode-locked solid state lasers [18].

\section{6}

\section{Photoelectron Spectroscopy}

Photoelectron spectroscopy is the measurement of electrons energy (emitted due to the photoelectric effect) when high energy electromagnetic radiation interacts with matter. Photoelectron spectroscopy is the most direct method of investigating electronic structure. The orbital structure of a molecule is reflected in the band structure of a photoelectron spectrum. The two main types of photoelectron spectroscopy are X-ray photoelectron spectroscopy and ultraviolet photoelectron spectroscopy. These spectroscopies depends on whether the ionization energy is provided by the X-ray photons or ultraviolet photons. Regardless of the incident photon beam, all photoelectron spectroscopies revolves around the general theme of surface analysis by measuring the ejected electrons.

The best way of measuring the stoichiometry of TMDs is by X-ray 
photoelectron spectroscopy (XPS). XPS was employed to analyse the chemical compositions of the doped 2D-TMDs alloys like $\mathrm{Mo}_{1-x} \mathrm{~W}_{x} \mathrm{~S}_{2}$ [125], $\mathrm{WS}_{2(1-x)} \mathrm{Se}_{2}$ [126], and $\mathrm{Mo}_{1-x} \mathrm{~W}_{x} \mathrm{Se}_{2}$ [127]. By studying the core level binding energies of S2p, Mo3d, and W4f peak intensities they have estimated the doping levels in the TMDs alloys. Just like Raman and PL spectroscopies the XPS has shown layer dependent shift in $\mathrm{MoS}_{2}$ thin films [128]. The binding energy shifts downward $\sim 0.2 \mathrm{eV}$ as the film thickness increases from 3 to 10 layers. The XPS has also proved a reliable tool in studying the structural defects in CVD grown $\mathrm{MoS}_{2}$ [129]. 


\section{3 \\ Experimental Methodology}

This chapter includes a detailed explanation of atmospheric pressure chemical vapor deposition process used for the synthesis of TMDs. Various techniques employed to characterize the grown samples, including Raman spectroscopy, photoluminescence, atomic force microscopy, X-ray photoelectron spectroscopy, second harmonic generation, device fabrication, and electrical measurements. The experimental setups of all the characterization techniques are explained in detail.

\section{1}

\section{Chemical Vapor Deposition}

Chemical vapor deposition is a process used in the semiconductor industry for depositing high quality and high performance solid thin films on wafers substrates. In a typical CVD process, the substrate is exposed to one or more volatile precursor gasses, which form a solid layer by either reacting with or decomposing on the surface. The substrate surface is heated and chemical reaction occurs in vapor form and finally, a solid material is deposited on the substrate. By using a CVD process it is possible to deposit amorphous, polycrystalline epitaxial thin films or a single crystalline film on a wafer surface. There are many kinds of CVD processes, ranging from low pressure to atmospheric pressure to plasma-enhanced deposition.

In 2009, Xuesong Li et al. [130] reported a breakthrough in the growth of single layer graphene by CVD. In 2012, Yi-Hsien Lee et al. [17] synthesized large-area single layer $\mathrm{MoS}_{2}$ for the first time by CVD on $\mathrm{SiO}_{2} / \mathrm{Si}$ substrates. Nowadays CVD plays a vital role in the synthesis of Carbon nanotubes [131], Boron Nitride nanostructures [132], Graphene [130], and many 2D-TMDs [15-18,20,28]. For graphene synthesis, it requires a metallic catalytic substrate, such as Copper or Nickle with a growth process occurring at a temperature of $\sim 1100^{\circ} \mathrm{C}[130,133]$. But, for the synthesis of atomically thin semiconducting TMDs, it can be grown directly on $\mathrm{SiO}_{2} / \mathrm{Si}$, quartz, or sapphire substrate, at a temperature $\sim 800-900^{\circ} \mathrm{C}[15-18,20,28,134-138]$.

In this thesis, we have used the atmospheric pressure chemical vapor deposition (APCVD) system to synthesize $\mathrm{MoS}_{2}, \mathrm{WS}_{2}$, and their heterostruc- 


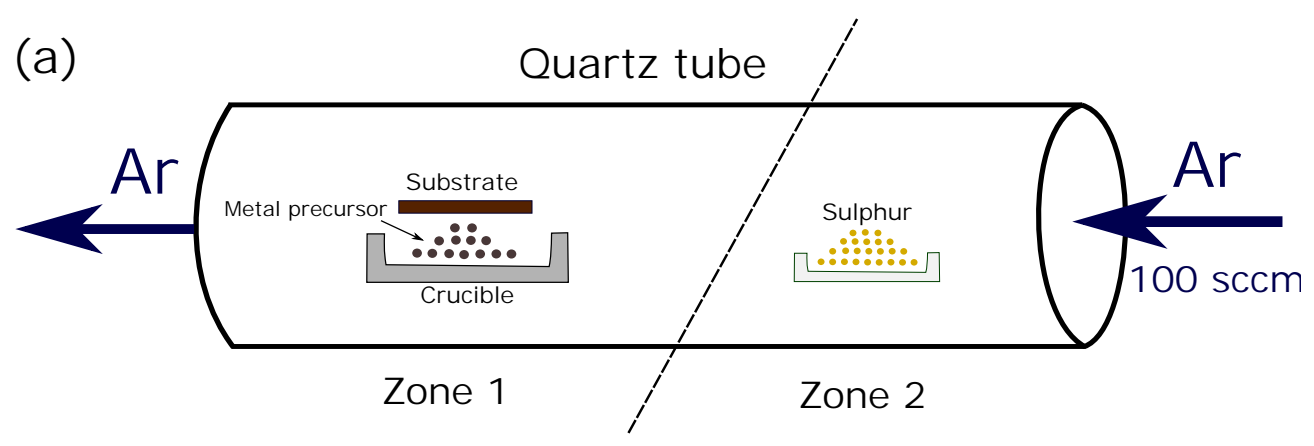

(b)

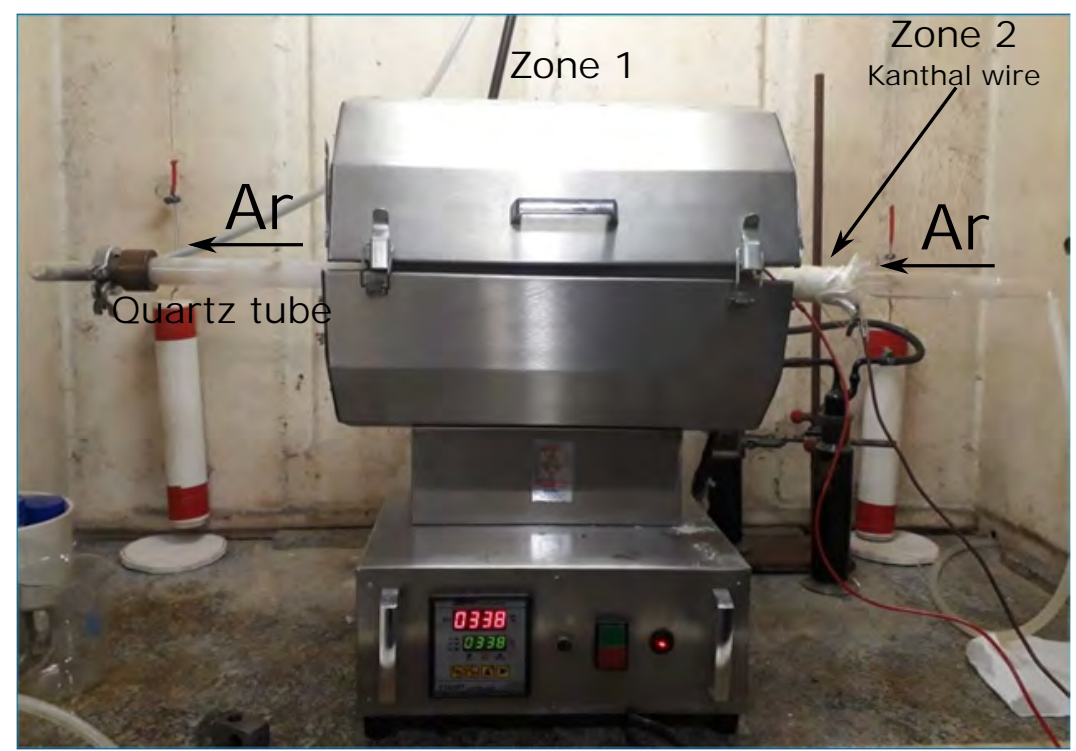

Figure 3.1: (a) Schematic diagram for the CVD synthesis of TMDs inside a quartz tube, showing the crucibles, and the substrate, two zones are heated independently at different temperatures. (b) CVD system at Protective Coatings and Nanostructured Materials Laboratory vandegraaff PUC-Rio.

tures. Figure 3.1(a) gives the schematic representation of CVD synthesis. The growth process is carried out inside a quartz tube in a dual-zone tubular furnace. Figure 3.1(b) shows a real tubular furnace (CVD), that we have at vandegraaff laboratory (PUC-Rio). However, this is not a dual zone furnace and we are manually heating zone 2 by wrapping a Kanthal wire around the quartz tube. Kanthal is an iron-chromium-aluminium alloy which is used in a wide range of resistance and high-temperature applications. By applying $\sim 3.5 \mathrm{~A}$ current to Kanthal wire we can reach to $\sim 350^{\circ} \mathrm{C}$. 


\subsection{1}

\section{Preparation of the Substrate}

The preparation of a clean substrate is very important before the growth procedure. First of all, the substrate should be immersed in acetone and ultrasonicate for 15-20 minutes, then ultrasonicate again for 15 minutes in isopropyl alcohol. Finally, wash it with distilled water. It's better to avoid drying substrate with air blows because it will let small dirt particles stick on the substrate resulting in the growth of dirty samples. When the substrate is clean, water will automatically slip from the substrate, but if necessary use $\mathrm{N}_{2}$ or Ar blow instead of air and avoid any stain on the substrate.

\subsection{2}

\section{Precursors and Crucible}

Precursor molecules are the molecules containing the element or elements which are the main source for the deposition of the film in the CVD process. In our synthesis we have used $\mathrm{MoO}_{3}$ (Sigma-Aldrich, 99.97\% pure), $\mathrm{WO}_{3}$ (SigmaAldrich, $99.99 \%$ pure), Tellurium powder (Sigma-Aldrich, $99.997 \%$ trace metals basis), and Sulphur fine powder (99.5\% pure) as precursor materials. The amount of the precursors depends on which TMD you are growing and on which substrate. The precursor's ratio, temperature, and substrate position are given in table 3.1 for every TMDs.

Crucible is a ceramic container in which metals or other substances may be melted or subjected to very high temperatures. We have used rectangular shape alumina crucible that can easily fit inside the quartz tube. Quartz crucible can also be used, but in our experience alumina crucible is more favourable, having high melting point $\left(\sim 2100^{\circ} \mathrm{C}\right)$. The precursor powders are loaded in the crucible and substrate is placed on the crucible. Figure 3.2(a) shows an alumina crucible and three different positions, showing how the substrate could be placed. The substrate's position is significant and depending on the TMD we are growing. For example, to grow monolayer $\mathrm{MoS}_{2}$ the substrate is placed on top of the crucible, while for $\mathrm{MoS}_{2} / \mathrm{WS}_{2}$ heterostructure the substrate should be placed in an incline position.

\subsection{3}

\section{Growth Process}

Place two crucibles inside the quartz tube one loaded with the metaloxide powder and the other with sulphur powder. Then place the quartz tube, in dual zone tubular furnace in a way that metal-oxide powder and the substrate is in zone 1 and sulphur in zone 2 , see figure 3.1. The distance between 
(a)

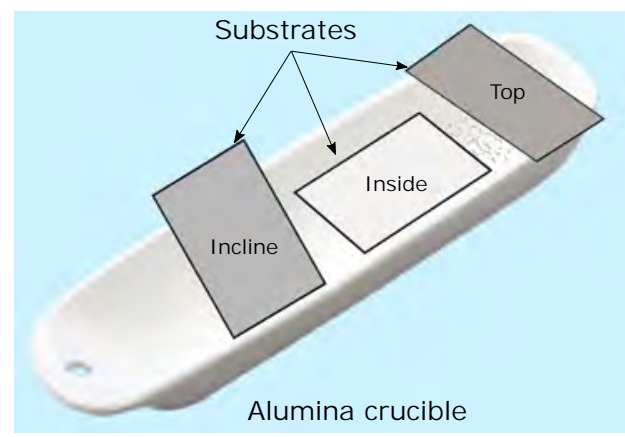

(b)

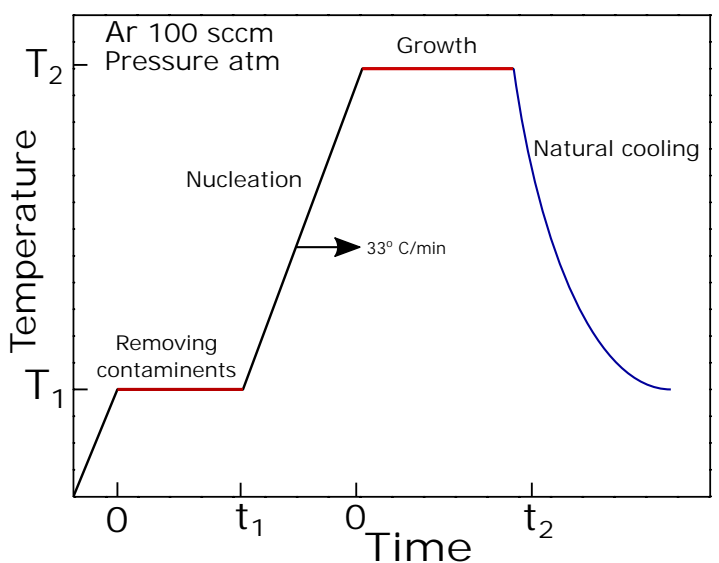

Figure 3.2: (a) Alumina crucible showing three different positions of the substrate. In every position substrate is facing downwards. (b) Temperature profile of the growth procedure, Ar flow rate is $100 \mathrm{sccm}$ during the whole process.

the two crucibles is $\sim 25 \mathrm{~cm}$. Use the Argon flow rate at $100 \mathrm{sccm}$ inside the quartz tube. During synthesis, first, heat the reaction chamber to $200^{\circ} \mathrm{C}\left(\mathrm{T}_{1}\right)$ in an Ar environment, and leave there for $20 \mathrm{~min}\left(\mathrm{t}_{1}\right)$. This will remove oxygen and other possible sources of contamination from the air. Now, heat the furnace independently at the rate of $33^{\circ} \mathrm{C} / \mathrm{min}$ until reaches $\mathrm{T}_{2}$ (depending on which TMD you are growing) for zone 1 , and $250^{\circ} \mathrm{C}$ for zone 2 (containing sulphur) at the same time. Then the materials will be kept under their respective constant temperatures for the next 15-25 min $\left(t_{2}\right)$ (depending on which TMD you are growing). Finally, let the furnace to cool down naturally. The furnace will take 2-3 hr to cool down, avoid the artificial way of cooling, or opening the furnace until temperature drops to $300^{\circ} \mathrm{C}$ otherwise, it will result in cracked samples. The flow rate of Ar should be kept constant at $100 \mathrm{sccm}$ during the whole growth process. Argon protects the system from oxygen and carries sulfur vapor from the upstream of the tube during the reaction, which causes it to react with the metal precursor and hence required samples are grown on the substrate. Figure 3.2(b) indicates the temperature profile of the growth procedure showing the ramp and two steps where the temperature should be kept constant.

Take an example of monolayer $\mathrm{MoS}_{2}$, we need 5-6 mg of $\mathrm{MoO}_{3}$ as a precursor material. The precursor powder is loaded in the crucible and the substrate is placed right on top of the precursor powder facing downwards. The growth temperature $\left(\mathrm{T}_{2}\right)$ and growth time $\left(\mathrm{t}_{2}\right)$ is $850^{\circ} \mathrm{C}$ and $15 \mathrm{~min}$. 


\subsection{4}

\section{Synthesis of Bilayers $\mathrm{MoS}_{2}, \mathrm{WS}_{2}$, and $\mathrm{MoS}_{2} / \mathrm{WS}_{2}$ Vertical Heterostruc- tures}

The faster rate of precursor sulfurization would increase the growth rate and results in bilayer samples. One way of increasing the growth rate is the addition of tellurium. The addition of tellurium enables the growth of extralarge $\mathrm{MoS}_{2}$ triangles with multiple layers $[28,139]$. Here tellurium is a catalyst it does not changes the composition of $\mathrm{MoS}_{2}$, it is removed by the Ar. To grown bilayer $\mathrm{MoS}_{2}, 5 \mathrm{mg}$ of $\mathrm{MoO}_{3}$ should be mixed with $15 \mathrm{mg}$ of tellurium powder. Temperature $\left(\mathrm{T}_{2}\right)$ and growth time $\left(\mathrm{t}_{2}\right)$ will be $850^{\circ} \mathrm{C}$ and $15 \mathrm{~min}$.

To grow bilayers $\mathrm{WS}_{2}$, relatively high amount (10-12 mg) $\mathrm{WO}_{3}$ should be used. Here, the substrate position is significant. The substrate should be placed inside the crucible as close as possible to the powder. The growth temperature $\left(\mathrm{T}_{2}\right)$ is $900^{\circ} \mathrm{C}$ and growth time $\left(\mathrm{t}_{2}\right)$ is $20 \mathrm{~min}$ for bilayers $\mathrm{WS}_{2}$. Controlling the bilayer is still challenging and it keeps growing multiple layers and layers with different stacking orientations. However, using the preceding steps we can achieve good bilayer crystals on the substrate.

To grow $\mathrm{MoS}_{2} / \mathrm{WS}_{2}$ vertical heterostructure the precursor powders $\mathrm{MoO}_{3}, \mathrm{WO}_{3}$, and Te should be mixed in a mortar pestle in the ratio of 1:1:3. The substrate position is inclined in this case. $\mathrm{T}_{1}$ is $200^{\circ} \mathrm{C}$ for $20 \mathrm{~min}\left(\mathrm{t}_{1}\right)$ and is the same for every synthesis, while the temperature $\left(\mathrm{T}_{2}\right)$ and growth time $\left(\mathrm{t}_{2}\right)$ is $850^{\circ} \mathrm{C}$ and $15 \mathrm{~min}$. Figure 3.3 shows the optical images of some TMDs grown by CVD, on $\mathrm{SiO}_{2} / \mathrm{Si}$ and quartz substrate. Table 3.1 shows a summary of the growth parameters for each TMDs. The best substrate positions are mentioned after many trials. For example if we put substrate inside the crucible while growing $\mathrm{MoS}_{2}$ it will result in dirty sample, if we put substrate on top position while growing $\mathrm{WS}_{2}$ growth will not happen.

(a)

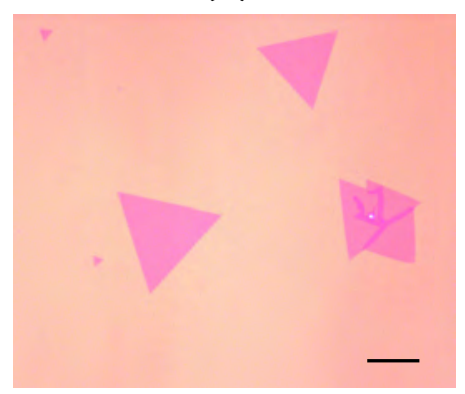

(b)

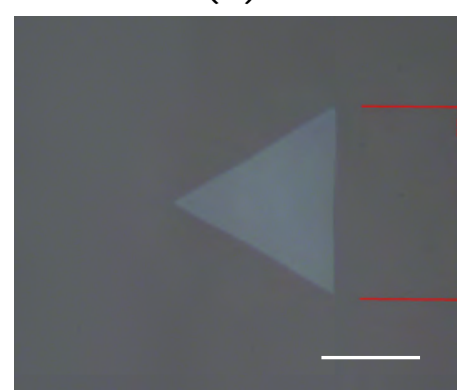

(c)

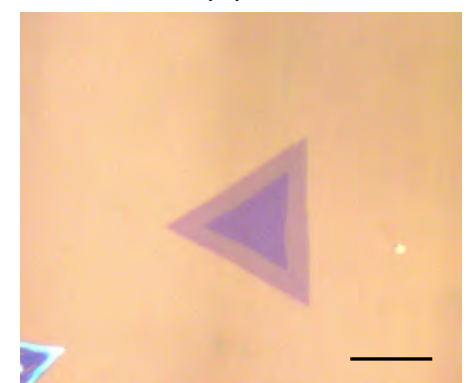

Figure 3.3: Optical images of some CVD grown TMDs. (a) $\mathrm{WS}_{2}$ monolayer on $\mathrm{SiO}_{2} / \mathrm{Si} 300 \mathrm{~nm}$. (b) $\mathrm{WS}_{2}$ monolayer on quartz. (c) Bilayer $\mathrm{MoS}_{2}$ on $\mathrm{SiO}_{2} / \mathrm{Si}$ $300 \mathrm{~nm}$. Scale bars are $10 \mu \mathrm{m}$ 


\begin{tabular}{|l||c|c|c|c|c|}
\hline Samples & Precursors & Ratio & $\begin{array}{c}\text { Temperature } \\
\left(\mathrm{T}_{2}\right)\end{array}$ & $\begin{array}{c}\text { Time } \\
\left(\mathrm{t}_{2}\right)\end{array}$ & $\begin{array}{c}\text { Substrate } \\
\text { position }\end{array}$ \\
\hline \hline Monolayer $\mathrm{MoS}_{2}$ & $\mathrm{MoO}_{3}$ & - & $800-850^{\circ} \mathrm{C}$ & $15 \mathrm{~min}$ & Top \\
\hline Monolayer $\mathrm{WS}_{2}$ & $\mathrm{WO}_{3}$ & - & $850^{\circ} \mathrm{C}$ & 17 min & Incline \\
\hline Bilayer $\mathrm{MoS}_{2}$ & $\mathrm{MoO}_{3}+\mathrm{Te}$ & $1: 3$ & $850^{\circ} \mathrm{C}$ & $15 \mathrm{~min}$ & Top \\
\hline Bilayer $\mathrm{WS}_{2}$ & $\mathrm{WO}_{3}$ & - & $900^{\circ} \mathrm{C}$ & $20 \mathrm{~min}$ & Inside \\
\hline $\mathrm{MoS}_{2} / \mathrm{WS}_{2}$ & $\mathrm{MoO}_{3}+\mathrm{WO}_{3}+\mathrm{Te}$ & $1: 1: 3$ & $850^{\circ} \mathrm{C}$ & $15 \mathrm{~min}$ & Incline \\
\hline
\end{tabular}

Table 3.1: Growth parameters for different TMDs. In all synthesis powder is placed into the boat with the substrate facing down.

\section{2}

\section{Optical Microscope}

An optical microscope uses visible light and a combination of lenses to see the magnified images of small objects. It allows us to see the objects which are too small for naked eyes, such as cells. The working of an optical microscope is very simple, it consists of a light source and a series of lenses see figure 3.4(a). Light is focused on the specimen by condenser lens then it enters the objective lens where it is magnified to produce a real image. The real image is then magnified again by the ocular lens to produce a virtual image that can be seen by eyes.

ZEISS Axio Scope. A1 (figure 3.4(b)) was used to see the magnified images of our samples. It composes of a movable stage that can move in $\mathrm{x}$, $\mathrm{y}$, and $\mathrm{z}$ direction. This model has five objective lenses $(5 \times, 10 \times, 20 \times, 50 \times$ and $100 \times$ ) and an ocular lens. The image can be seen on the computer by transferring the real image produced by the objective lens towards the camera which is connected to the computer. Some optical images taken from this microscope with a $100 \times$ lens are shown in figure 3.3. The visibility of TMDs on a substrate is the result of the difference between the reflectance of visible light from TMDs and the underlying substrate. 
(a)

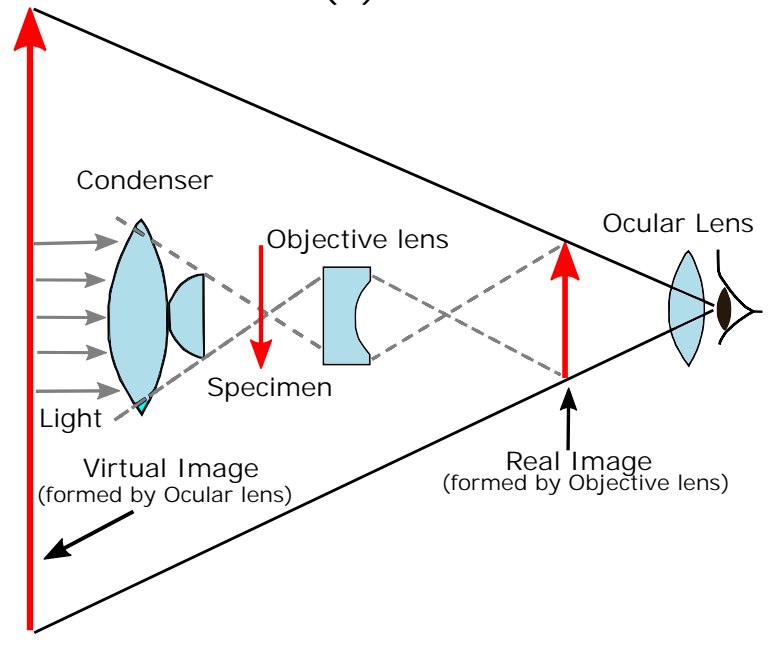

(b)

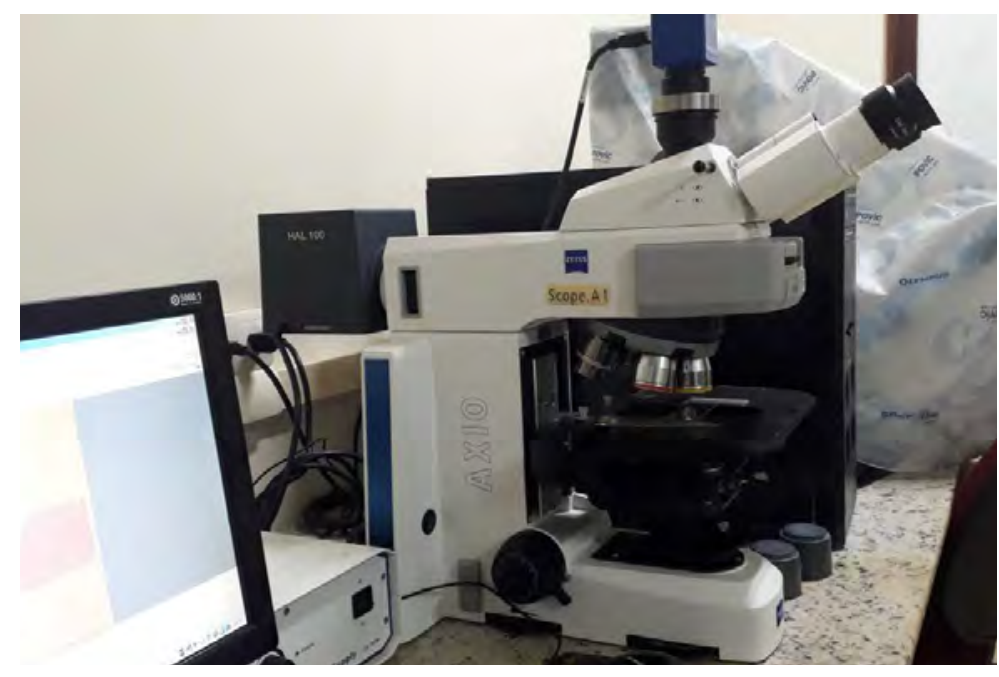

Figure 3.4: (a) Schematic of an optical microscope image formation. (b) ZEISS Axio Scope. A1 at Protective Coatings and Nanostructured Materials Laboratory Vandegraff PUC-Rio.

\section{3}

\section{Scanning Electron Microscope}

Scanning electron microscope (SEM) is a type of microscope that creates the image of a sample by scanning it at a very high energy beam of electrons. The electrons are continuously interacting with the atoms and produce, signals that contain information about the sample surface and bulk, composition, and electrical conductivity. The electron beam is usually, scan in a raster scan pattern, and the position of the beam is combined with the detected signal to produce an image. It gives us the opportunity to see the objects up to few nanometers. Specimens can be observed in high vacuum, in low vacuum, in wet conditions, and at a wide range of cryogenic or elevated temperatures. SEM is 
useful in understanding the surface of the sample.

(a)

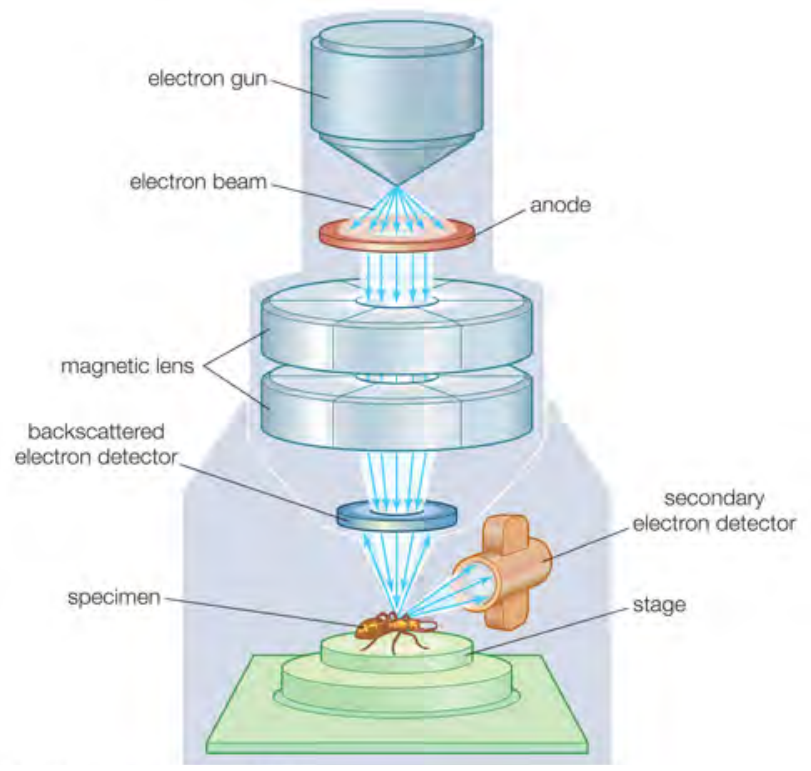

(b)

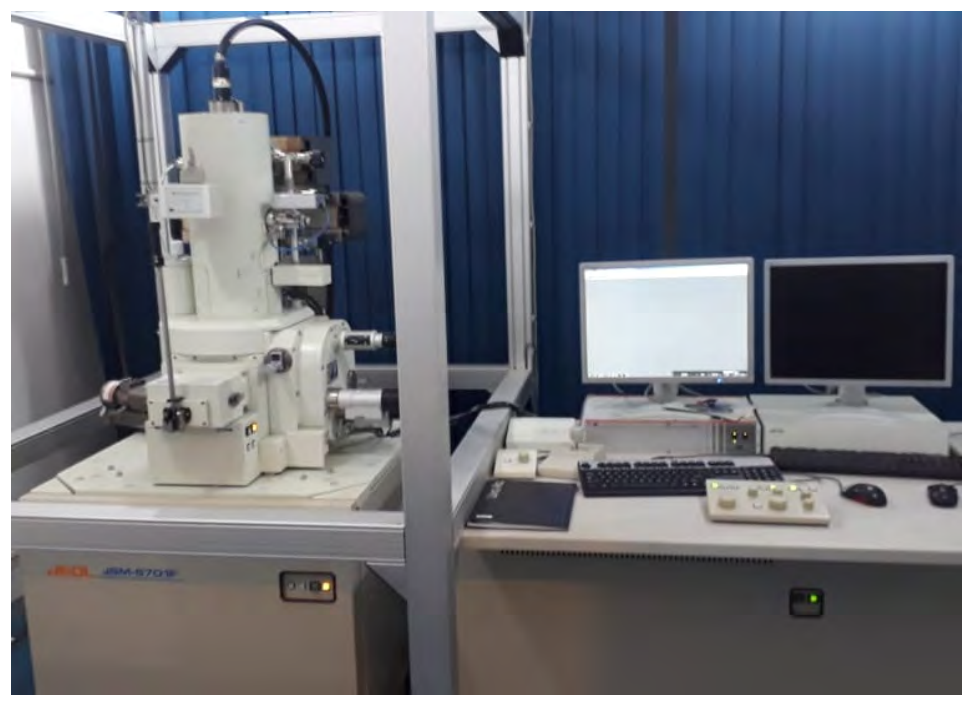

Figure 3.5: (a) Schematic of a SEM image formation. Adopted from [140]. (b) JEOL JSM-7610F Field Emission SEM at vandegraaff PUC-Rio.

In a typical SEM, an electron beam is emitted from an electron gun fitted with a filament cathode made up of tungsten. Tungsten is normally used because it has a very high melting point and lowest vapor pressure in all metals, hence allowing it to be heated for electron emission. The electron beam, which has an energy of $0.2-40 \mathrm{keV}$, is focused by two magnetic lenses to a spot of $0.4-5 \mathrm{~nm}$ in diameter, see figure 3.5(a) [140]. The beam passes through pairs of scanning coils or pairs of deflector plates in the electron column, typically in the final lens, which deflects the beam in the $\mathrm{x}$ and $\mathrm{y}$ axes so that it scans 
in a raster fashion over a rectangular area of the sample surface.

Now when the primary electron beam interacts with the sample, the electrons lose energy by repeated random scattering and absorption within a teardrop-shaped volume of the specimen known as the interaction volume, which extends from less than $100 \mathrm{~nm}$ to approximately $5 \mu \mathrm{m}$ into the surface. The energy exchange between the electron beam and the sample results in the reflection of high-energy electrons by elastic scattering, emission of secondary electrons by inelastic scattering, and the emission of electromagnetic radiation, each of which can be detected by specialized detectors. The beam current absorbed by the specimen can also be detected and used to create images of the distribution of specimen current. Electronic amplifiers of various types are used to amplify these signals, which are displayed as variations in brightness on a computer monitor. Each pixel of computer video memory is matched with the position of the beam on the specimen in the microscope, and the resulting image is, therefore, a distribution map of the intensity of the signal being emitted from the scanned area of the specimen. Finally, machines image is saved to a computer's data storage. The JEOL JSM-7610F Field Emission SEM was used in our work (figure 3.5(b)), which offers magnification with $0.8 \mathrm{~nm}$ resolution at $15 \mathrm{kV}(1.0 \mathrm{~nm}$ at $1 \mathrm{kV})$ and unmatched beam stability, making it possible to observe the fine surface morphology of our samples.

\section{4}

\section{Raman Spectroscopy}

Before we discuss the Raman spectroscopy it is important to understand the phenomena behind the Raman scattering.

\subsection{1}

\section{Raman Effect}

Whenever the light (photons) interacts with a medium different processes may occur, such as transmission, reflection, absorption, or luminescence. However, a small fraction of incident light (photons) is scattered because of inhomogeneities inside the medium. The phenomenon of scattering of light from the medium is known as Raman effect first discovered by Sir C.V.Raman in 1928 which earned him the Nobel Prize in 1931 [141]. He has observed when light (photons) are scattered from an atom or a molecule, most of them are elastically scattered which is called Rayleigh scattering, such that the scattered photons have the same energy (frequency and wavelength) as the incident photons. A small fraction of scattered photons, about one in a million, has a difference in energy. This is attributed to the energy absorption by atoms 
or molecules and the energy difference was related to the chemical structure of the molecule responsible for the spread. Later on, the Raman effect has become an important tool for analyzing the chemical composition of solids, liquids, and gases. Raman spectrum provides a chemical "fingerprint" of the substance. Using Raman spectroscopy technique one can distinguish between a metallic carbon nanotube, semiconductor, doped graphene samples, disorder in the structure, type of edge (zig - zag and armchair), deformation and tension in the crystal, oxidation, amorphization, number of layers and coupling between layers [142]. So, the Raman process is the starting point for understanding any type of change in the peaks (or band) of the Raman spectrum of the samples.

\subsection{2}

\section{Theory of Raman Scattering}

Consider a photon of energy $\mathrm{h} \nu$ collides with a molecule having energy $\mathrm{E}_{i}$. Photons scatter after colliding with the molecule with frequency $\nu_{o}$ and energy $\mathrm{h} \nu_{o}$, while the molecule will have the final energy as $\mathrm{E}_{f}$. The change in velocity of a photon before and after the collision is negligible, thus by the law of conservation of energy:

$$
\begin{gathered}
E_{i}+h \nu=E_{f}+h \nu_{o} \\
\Longrightarrow \nu_{o}=\nu+\left(E_{i}-E_{f}\right) / h
\end{gathered}
$$

From this equation can have three cases.

- If $E_{i}=E_{f}$ then $\nu_{o}=\nu$, which means that photon is simply deflected from the molecule without losing or gaining energy from it (Elastic collision). This corresponds to Rayleigh scattering.

- If $E_{i}<E_{f}$ then $\nu_{o}<\nu$, which means photon gives its energy to the molecule and hence the scattered photon have less energy then the incident photon. This corresponds to stokes lines.

- If $E_{i}>E_{f}$ then $\nu_{o}>\nu$, which means the molecule was already in excited state and photon absorbs energy from the molecule and resulting in high frequency scattered photon. This corresponds to anti-stokes lines.

From 3-2 we get

$$
\begin{aligned}
& \nu_{o}=\nu+\delta E / h \\
& \Longrightarrow \nu_{o}-\nu=\delta \nu
\end{aligned}
$$

Hence in Raman scattering, the frequency difference of the scattered photon corresponds to the characteristic frequency $\delta \nu$ of the molecule. The stokes and 
anti-stokes lines should be equidistant from the Rayleigh line on either side. The process is illustrated in figure 3.6.

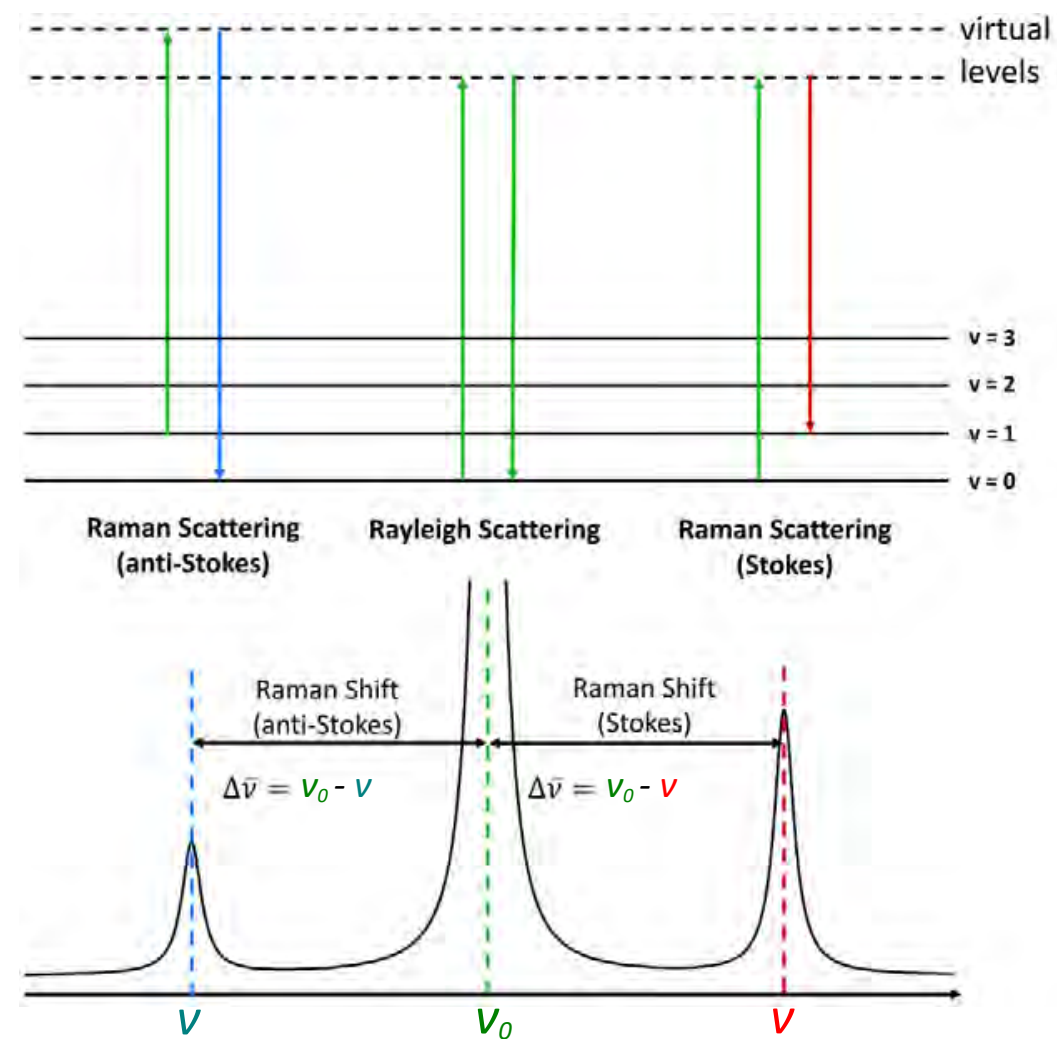

Figure 3.6: Stokes and anti-Stokes Raman scattering.

\subsection{3}

\section{Raman Spectrometer}

Raman spectroscopy is a non-destructive characterization tool and is applicable both in the laboratory and in industrial scale. Raman spectroscopy has become one of the most popular techniques for characterizing $2 \mathrm{D}$ materials [143]. It works on the principle of Raman scattering after scattering from the sample Raman spectra is obtained. Raman spectra is a measure of the Stokes process, plotting the intensity of the scattered light as a function of the Raman shift. Raman shift is the difference between the energy of the incident photon and the scattered photon. The units of this displacement are $\mathrm{cm}^{-1}(1 \mathrm{meV}=$ $8.0655447 \mathrm{~cm}^{-1}$ ). Figure 3.7(a) shows the schematic of a Raman spectrometer, here we discuss briefly each component and its function step by step.

Laser Laser produces a high power monochromatic light, one can use different wavelength lasers. The laser is incident on the sample under consideration, to activate its vibrational modes. 
(a)

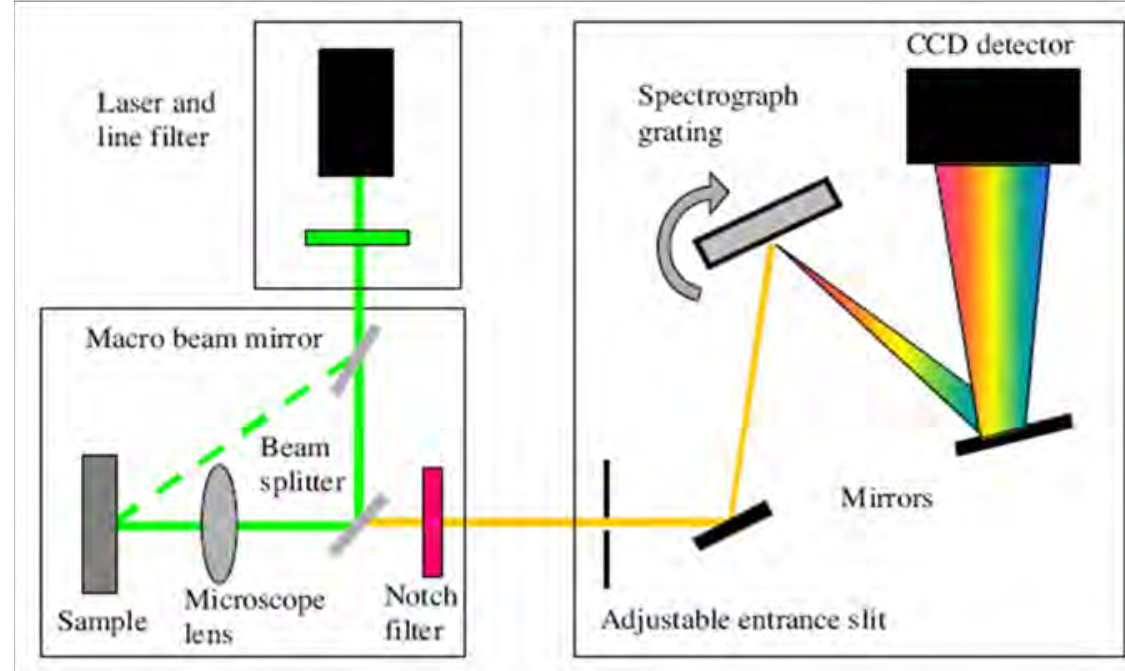

(b)

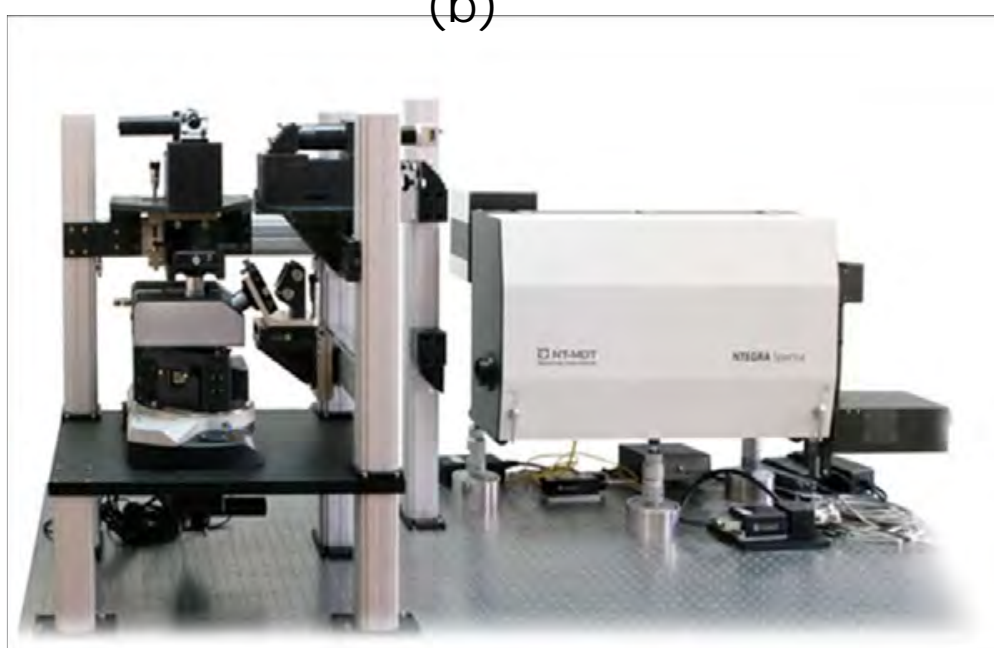

Figure 3.7: (a) Schematics of Raman spectrometer. (b) NT-MDT, NTEGRA Spectrometer at Protective Coatings and Nanostructured Materials Laboratory Vandegraaff PUC-Rio.

Lens It is used to focus the incident laser beam on the sample.

Notch filter A notch filter is a band-stop filter with a narrow stop-band. It passes most frequencies without any change through it but attenuates those in a specific range to a very low level. Scattered laser at the wavelength corresponding to the incident laser line (Rayleigh scattering) is filtered out by notch filter, while the rest of the collected light is dispersed onto a detector.

Grating Grating is the most important component in the Raman spectrometer. It disperses the signal coming from the sample onto the CCD detector by deflecting each wavelength at a different angle. It is made up of many closely 
spaced parallel lines (grooves) which permit diffraction gratings to disperse the components of light into separate wavelengths. The number of grooves $/ \mathrm{mm}$ determines the dispersion characteristics. A high number of grooves/mm results in a high dispersion and thus a high resolution, by distributing the signal over a larger number of CCD pixels. Generally, Raman spectrometer has different grating with different ranges which can be changed according to the sample under consideration.

CCD (charged-coupled device) The CCD camera is the last element in the detection beam path. It converts the photons that reach the chip into electronic signals that can be used to draw the spectra on a computer. This is an important component of a Raman spectrometer and choosing the correct CCD strongly affects the performance of the instrument. The Raman spectra we obtained on the computer shows some peaks at different frequencies, which is a chemical "fingerprint" of the sample and contains the structural information about the substance.

We have performed Raman spectroscopy using a micro-Raman spectrometer (NT-MDT, NTEGRA SPECTRA) equipped with a CCD detector and a solid state laser, with a wavelength of $473 \mathrm{~nm}(2.62 \mathrm{eV})$ shown in figure 3.7(b). We performed the experiments using a $100 \times$ magnification objective, giving a laser spot of the order of $\sim 1 \mu \mathrm{m}^{2}$ diameter and a numerical aperture of 0.7. The spectrometer is equipped with a piezoelectric ceramic with all the electronics of a microscope, it allows to make Raman maps over an area of 30 $\times 30 \mu \mathrm{m}^{2}$, with a step size of $1.0 \mu \mathrm{m}$. To study the defects XploRA Confocal Raman Microscope - HORIBA equiped with three internal lasers (532, 638 and $785 \mathrm{~nm}$ ) was used. 532 and $638 \mathrm{~nm}$ laser was used to study the second order Raman peaks. The single spectra were acquired with the $100 \times$ objective lens which produces a laser spot of $0.9 \mu \mathrm{m}^{2}$ using a 1800-line/mm grating.

\section{5}

\section{Photoluminescence Spectroscopy}

Photoluminescence spectroscopy is a common technique used to characterize the optoelectronic properties of semiconductors and other materials. Consider a sample absorbs a photon of the incoming light to promote an electron from the ground state in a molecule or (valence band in a solid) to the first excited molecular electronic state or (conduction band in a solid), respectively. After the electron is in the excited state (conduction band), it returns to the ground state (valence band), as a result, a photon can be emitted in the form of electromagnetic radiation. This emission process is called photoluminescence. 
The experimental setup of photoluminescence spectroscopy is very simple, a laser tuned to a wavelength close to the bandgap energy of the sample is directed onto the sample. When the laser beam is incident on the sample, photoluminescence occurs and light (photons) is emitted from the sample at wavelengths dependent on the sample composition. The emitted light is directed on the detector and passed into a fiber optic cable and then into a spectrometer. Since the incident laser light is also collected and it usually has a significantly higher intensity, a filter is placed in front of the fiber input to remove the incident laser light. Inside the spectrometer, a diffraction grating diffracts different wavelengths in different directions towards an array of photodetectors that measure the intensity of each wavelength component. This wavelength is then passed to CCD which converts the photons that reach the chip into an electronic signal. The digital information is interpreted by the computer, which can display a PL spectrum. The spectrum indicates the relative intensities of light of different wavelengths entering the detector. Figure 3.8 shows the schematic diagram of the PL experimental setup.

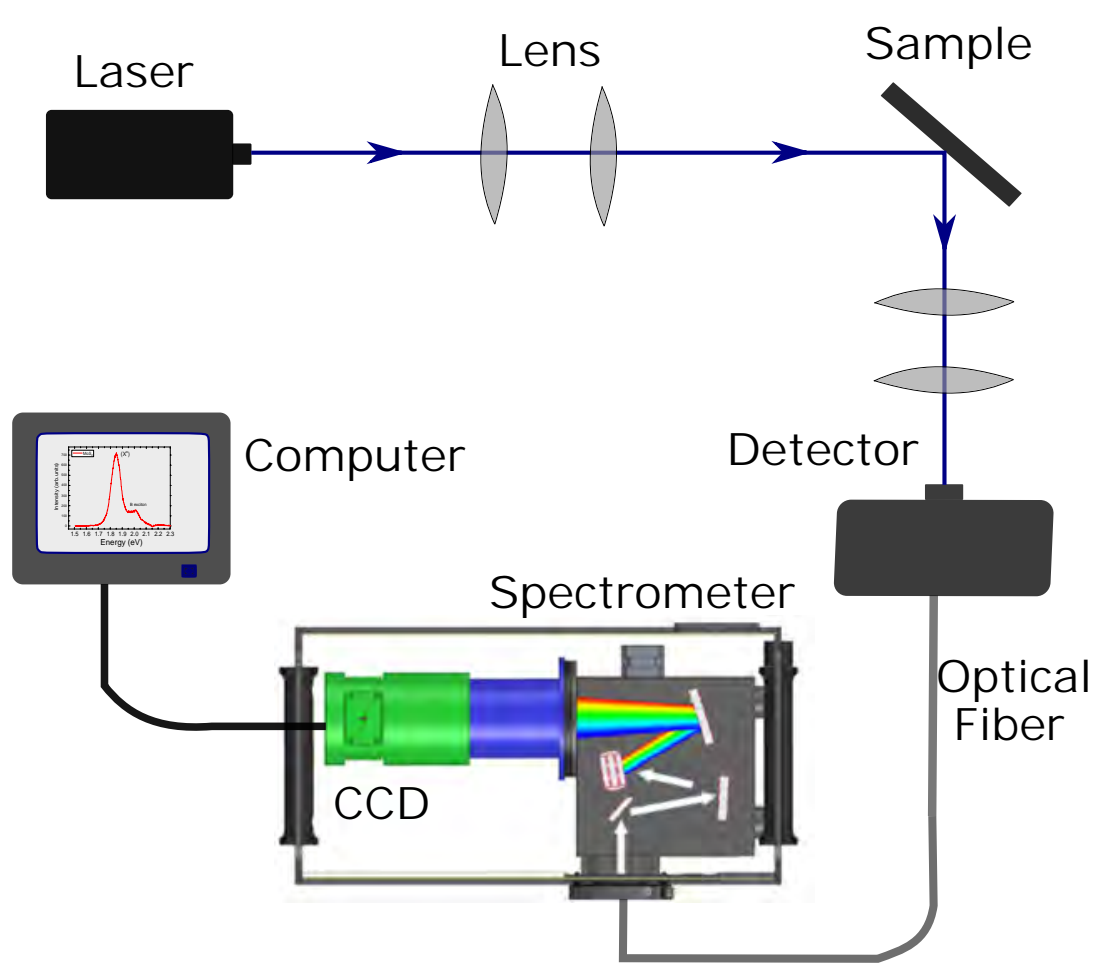

Figure 3.8: Schematic diagram of the PL experimental setup.

Mostly in TMDs compared to PL, Raman scattering is a weak phenomenon and that is sometimes the reason for being unable to acquire a Raman spectrum. Raman spectrometer is ideal for performing laser-excited photoluminescence [144]. We performed the photoluminescence spectroscopy using the 
same NT-MDT Raman equipment (figure 3.7(b)). The PL can be measured by changing the grating to $150 / 600$ grooves $/ \mathrm{mm}$.

\section{6}

\section{Atomic Force Microscopy}

Atomic force microscopy (AFM) is a technique used to generate images at extremely high resolution and examine features at the atomic level. This makes AFM a perfect technique to characterize TMDs layers. AFM image gives us the surface morphology of a sample, which can be used to measure the exact thickness of a sample. Moreover, this high-resolution AFM image can also give the thickness variation and the distribution of contaminants across the entire surface. AFM is based on the interaction between a tip and the sample surface. This interaction allows us to obtain information about the topography of the sample surface. The interaction force between the tip and the surface is like Van der Waals force. When the distance between the tip and the surface is very small, the electronic orbitals of the surface and tip atoms start repelling each other. AFM can be used for imaging any conducting or non-conducting surface.

The AFM microscope consists of a sharp tip which runs on the sample surface. The interaction force between the atoms of the surface and tip is measured by deflecting a cantilever where the tip is fixed. A diode laser is focused at the end of the cantilever, the laser beam is reflected on a position sensitive photodetector. As the tip moves up and down due to the surface, the cantilever deflects and the intensity changes in the position of four quadrants photodetector. These changes in the position of the tip are recorded by the detector. Using software from the equipment itself, it converts this data into an image based on the position of the cantilever [145]. Generally, cantilever tips are made up of $\mathrm{Si}_{3} \mathrm{~N}_{4}$ or silicon.

Figure 3.9 shows a cantilever deflection detection scheme and a laser beam. Another important aspect of the AFM microscope's operation is the position how the sample is moved since very precise control over this displacement is needed. For this, a piezoelectric ceramic scanner is used which moves the sample in the $\mathrm{x}, \mathrm{y}$, and $\mathrm{z}$ directions. When an electrical voltage is applied the piezoelectric scanner undergoes a variation in its dimensions, thus controlling the displacement of the sample [146].

In this thesis, we have characterized our samples by contact mode AFM using the same NT-MDT equipment and a $10 \times 10 \mu \mathrm{m}^{2}$ piezo electric stage. Our NT-MDT is a multifunctional equipment and can be used to generate high quality AFM images [147]. The measurements were taken in contact mode with 


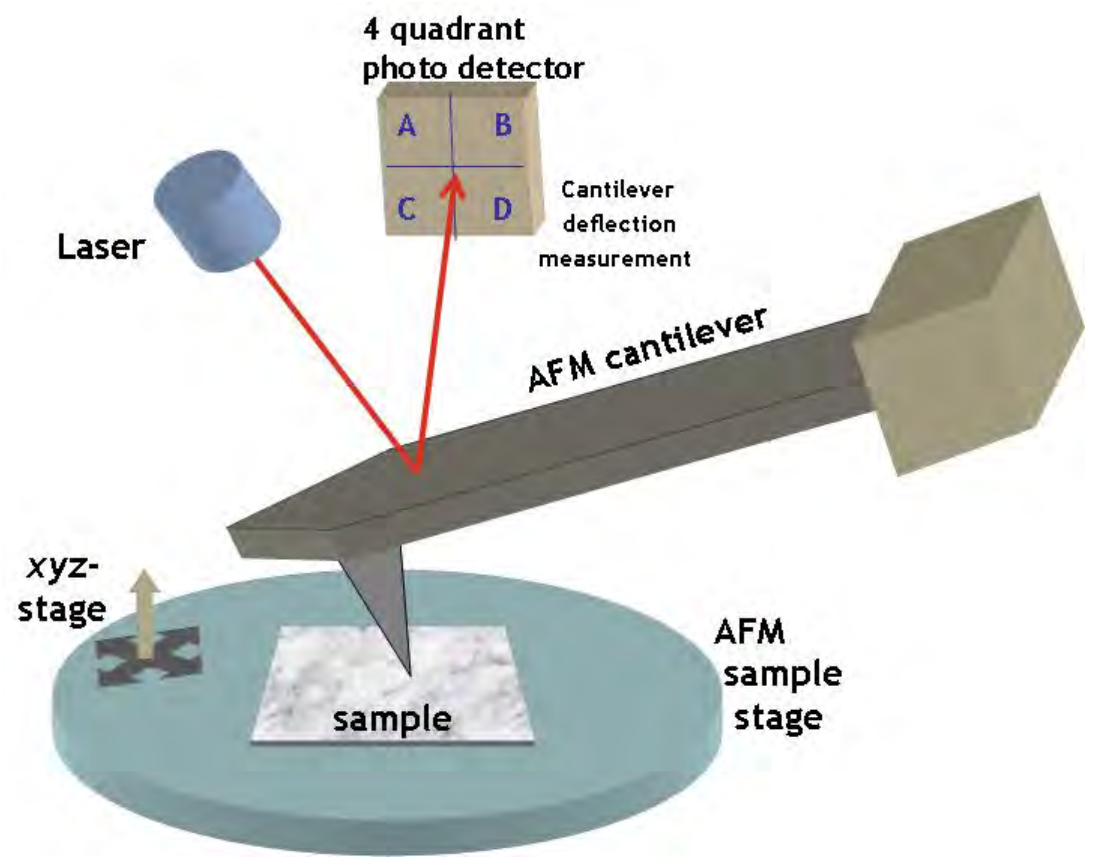

Figure 3.9: Cantilever deflection detection scheme by means of a beam of laser. Adopted from [146].

the silicon tip with gold on top of the cantilever .

\section{7}

\section{X-ray Photoelectron Spectroscopy}

The X-ray photoelectron spectroscopy (XPS) is a surface analysis technique widely used in the study of the composition of an element or a compound. XPS enables us to detect all elements in the periodic table except Hydrogen and Helium, due to the absence of core electrons [148]. This technique was developed by Kai Siegbahn in 1960s and is based on the application of the photoelectric effect. In photoelectric effect when high power electromagnetic radiation, hits a material, if the incident radiation energy is sufficient, an atom absorbing it will result in exciting an electron from the material [149]. The electrons emitted in this manner are called photoelectrons.

$$
X+h \nu \rightarrow X^{+}+e^{-}
$$

Here $\mathrm{X}$ is the atom in its fundamental state (containing $\mathrm{n}$ electrons), $\mathrm{h} \nu$ is the excitation energy, $\mathrm{X}^{+}$is the atom in its excited state after being photoionized (containing n-1 electrons) and $\mathrm{e}^{-}$is the emitted photoelectron. Similarly, when an X-ray beam is focused on the surface of the sample, the photoelectric effect occurs and photoelectrons are emitted from the sample. By analyzing the energy of the ejected photoelectrons one can determine the elements present in it. The energy of the photoelectron depends on the element from which it is 
originated and the orbital of the ground state in which the electron was found. The energies associated with this excitation, described by the photoelectric effect, can be represented as:

$$
h \nu=K \cdot E+B \cdot E+\phi_{o}
$$

Where $\mathrm{h} \nu$ is the X-ray energy absorbed, K.E is the kinetic energy of the photoelectron after being ejected by the atom, B.E is the electron binding energy, and $\phi_{o}$ is the work function of the surface (i.e the minimum energy required to remove an electron of the material, $\left.\phi_{o}=E_{\text {vacuum }}-E_{\text {Fermi }}\right)[150,151]$.

In an XPS experiment, the samples are placed in an ultra-high vacuum chamber and are irradiated by an X-ray beam. When the incident beam energy $(\mathrm{h} \nu)$ is greater or equal to the binding energy (B.E) of an electron at the core level of an atom, these electrons are emitted from the sample surface. These emitted photoelectrons are subsequently separated according to their kinetic energy and counted with the help of an electrostatic analyzer and collected by a detector. The schematic of an XPS setup is shown in figure 3.10 [152].

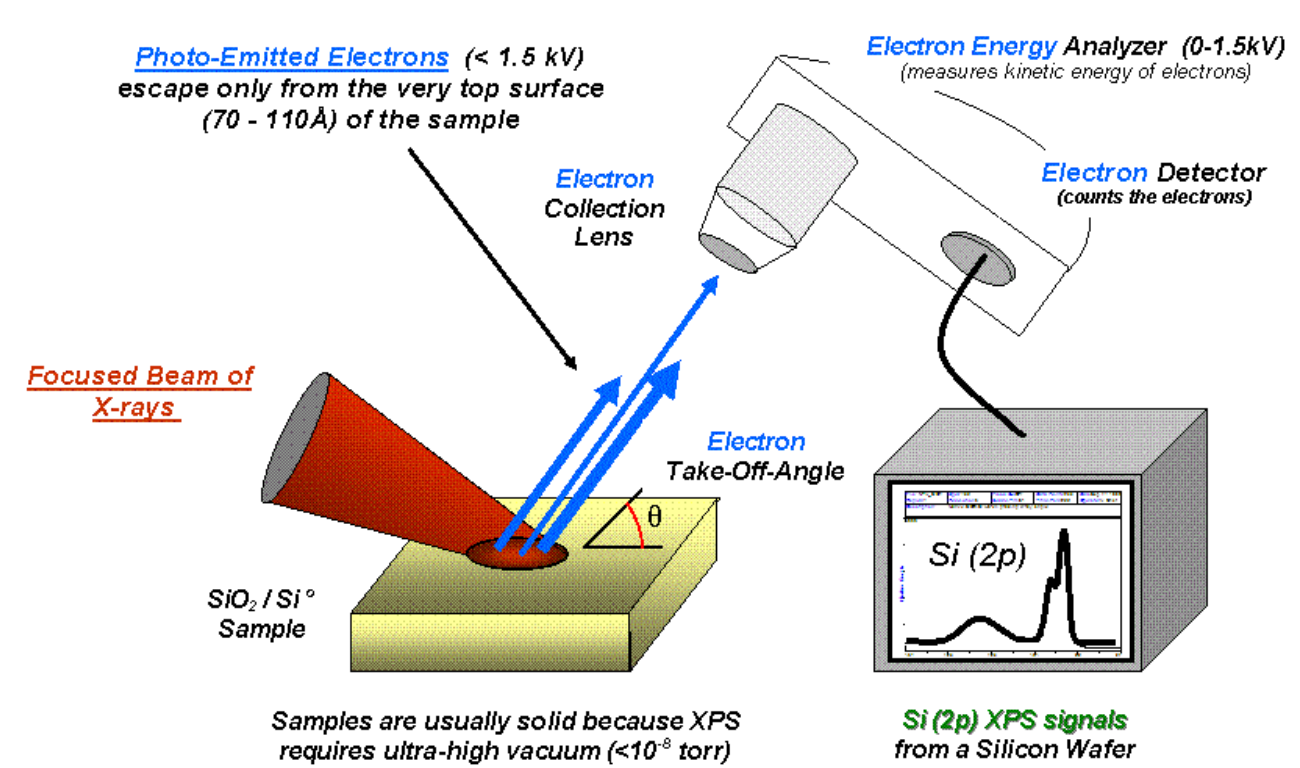

Figure 3.10: Experimental setup for XPS. Adopted from [152].

The XPS setup in vandegraaff laboratory (PUC-Rio) was used in this thesis work, the photoelectrons are filtered in energy with a VG Thermo Alpha 110 hemispherical analyser, later detected by 7 "channeltron" detectors that transform the electrons into an amplified current pulse so that they can be amplified. Finally, the spectrum is recorded as intensity (counts per second) vs. kinetic energy of photoelectrons or their binding energy. The X-rays source used to generate the photoelectrons in the sample was Al-K $\alpha(1486.6 \mathrm{eV})$. 
The measurements were performed with the analyser positioned normal to the surface of the sample. Data analysis was performed using the CasaXPS software (from Casa Software Ltd.), taking as a reference for the binding energies the peak of $1 \mathrm{~s}$ carbon at $284.6 \mathrm{eV}$. The XPS on our samples was performed by Prof. Marcelo da Costa.

\section{8}

\section{Introduction to Second Harmonic Generation}

Second harmonic generation is a nonlinear optical process in which two photons having the same frequency interact with a nonlinear material, giving rise to a new photon with twice the frequency of the initial photons. The second harmonic generation can occur in non-centrosymmetric materials or at the surface of a material with any symmetry group. However, It is forbidden in centrosymmetric materials. It was first demonstrated in crystalline quartz by P. Franken et al. in 1961 [153].

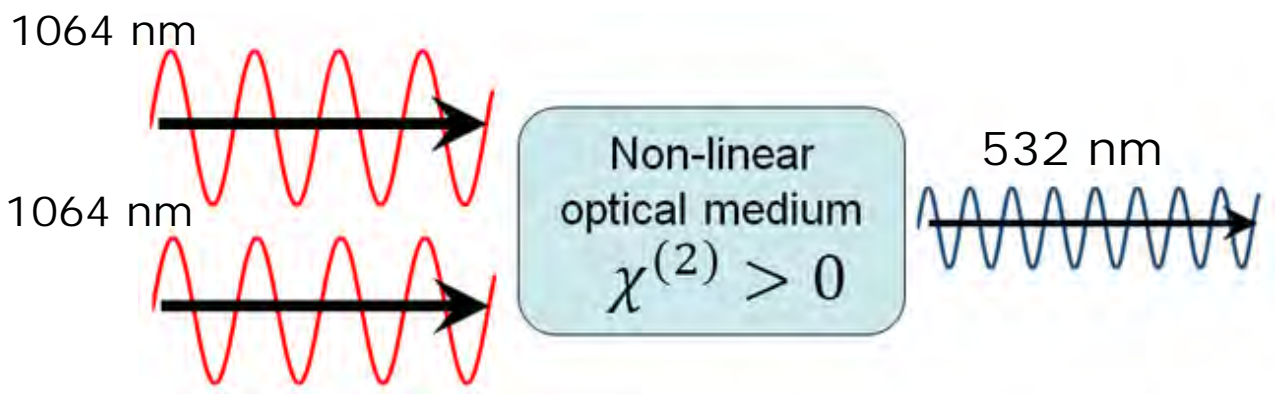

Figure 3.11: General second harmonic generation phenomena.

Figure 3.11 shows when two photons of higher wavelength pass through a nonlinear medium it gives a single photon of half of the wavelength of the initial photon. Second harmonic generation has many practical applications e.g. changing the colour of the laser is possible. Typical green laser $(532 \mathrm{~nm}$ ) is actually the SHG of Nd:YAG laser (1064 nm). Applying a non linear optical crystal KTP, it turns invisible Nd:YAG infrared laser into highly visible green laser. The radiated optical field in SHG is directly proportional to the nonlinear component of the electric polarization of the material, i.e:

$$
E_{o}(\omega) \propto P_{o}(\omega)
$$

Microscopically, the nonlinear process can be described by the lightinduced polarization expressed as:

$$
P_{o}(\omega)=\epsilon_{o} \chi^{(1)} E_{o}(\omega)
$$


here $\chi^{(1)}$ is the electrical susceptibility of the first order of the material, with the superscript index indicating linear treatment. In isotropic medium susceptibility is a scalar quantity. But due to the anisotropic nature of some materials, it is necessary to analyze susceptibility in tensorial form. In a system with a significantly intense optical field, to intra-atomic fields, polarization can be written as a series of powers of the electric field [154]:

$$
P_{o}(\omega)=\epsilon_{o}\left[\chi^{(1)} E_{o}(\omega)+\chi^{(2)} E_{o}(\omega)^{2}+\chi^{(3)} E_{o}(\omega)^{3}+\ldots \ldots . .\right]
$$

where $\chi^{(2)}$ and $\chi^{(3)}$ are the second and third order susceptibilities, respectively. The absence of second harmonic generation in centrosymmetric materials is due to the presence of inversion symmetry, the $\chi^{(2)}$ tensor is zero. For SHG to occur we need an even response of the polarization $\mathrm{P}$ to the electric field $\mathrm{E}$, i.e. the polarization will have the same direction for a certain electric field E. Obviously for symmetry reasons this is not possible if your lattice is centrosymmetric. The SHG is a fast and non-destructive method to characterize layered TMDs.

Similar way third harmonic generation (THG) is a process that generates light with one-third the wavelength. In THG three photons are destroyed, creating a single photon at three times the frequency. This process can be applied in signal processing, ultrafast laser production, and imaging. This process is generated by the third order non-linear response of the material, it can be used in the characterization of the third order response, regardless of the material will be centro symmetric or not. The THG process can be observed using the same experimental apparatus than the one used for SHG.

\subsection{1 \\ Second Harmonic Generation Measurements}

We have characterized our samples with second and third harmonic measurements. We have collaborated with Prof. Christiano's group at Mackenzie Presbiterian University, Sao Paulo. The experimental setup for second harmonic generation at Mackgraphe, Mackenzie Presbiterian University, SP is shown in figure 3.12. The experimental setup for the analysis can be divided into two branches. The first corresponds to the part responsible for the excitation and collection of the SHG signal. This setup branch contains a $1560 \mathrm{~nm}$ Erbium-doped fiber laser, with repetition rate of $89 \mathrm{MHz}$ and pulse duration of $150 \mathrm{fs}$. The beam is focused on the sample with normal incidence through a $20 \times$ objective lens, reaching a beam diameter of $\sim 3 \mu \mathrm{m}$ in the focused region.

The second harmonic is generated on a sample with the wavelength of $780 \mathrm{~nm}$. Then, we collected the signal in reflection. It is separated from the laser pump when passing through a low pass filter (dichroic mirror, Thorlabs, 
(a)

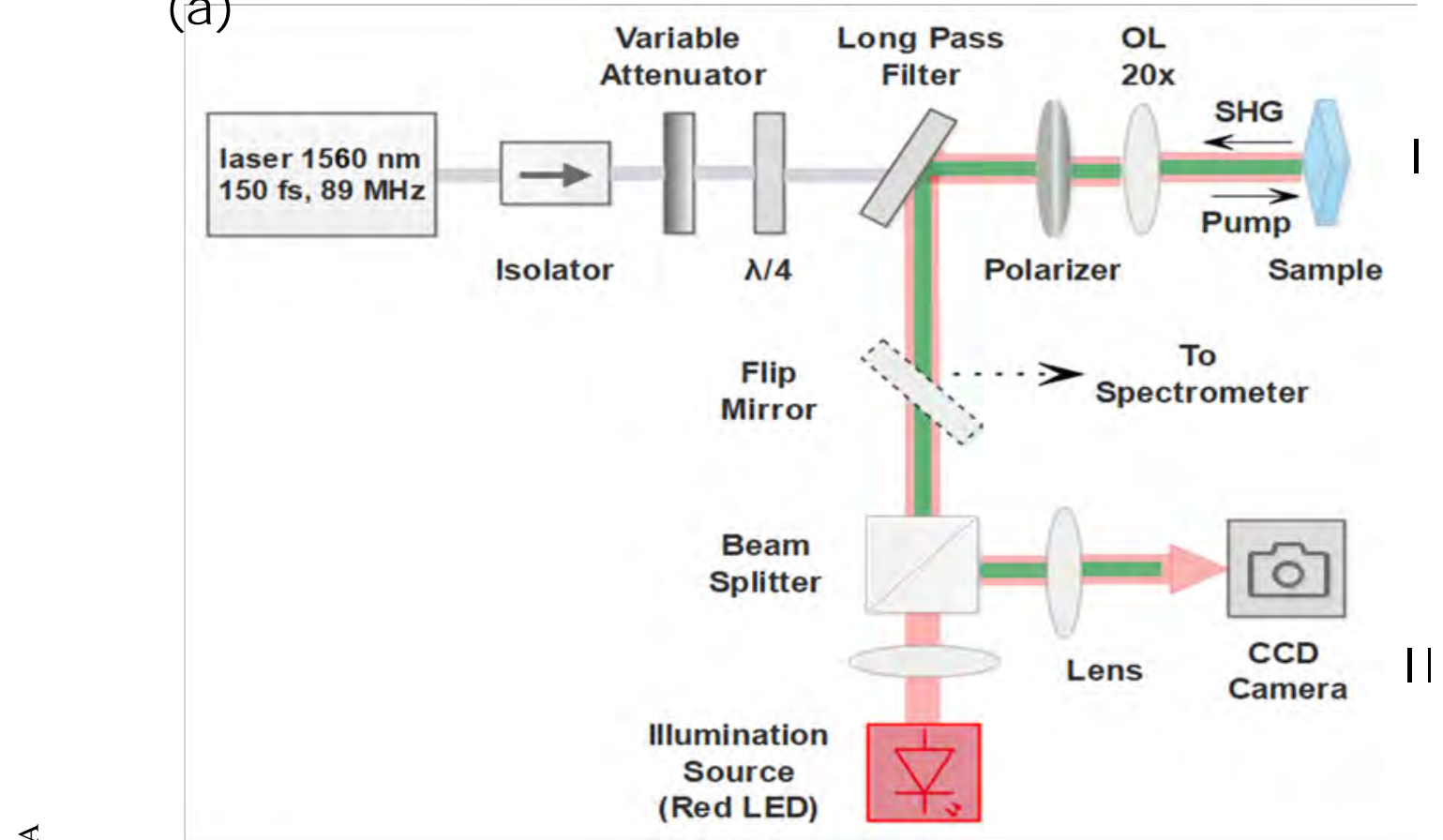

(b)

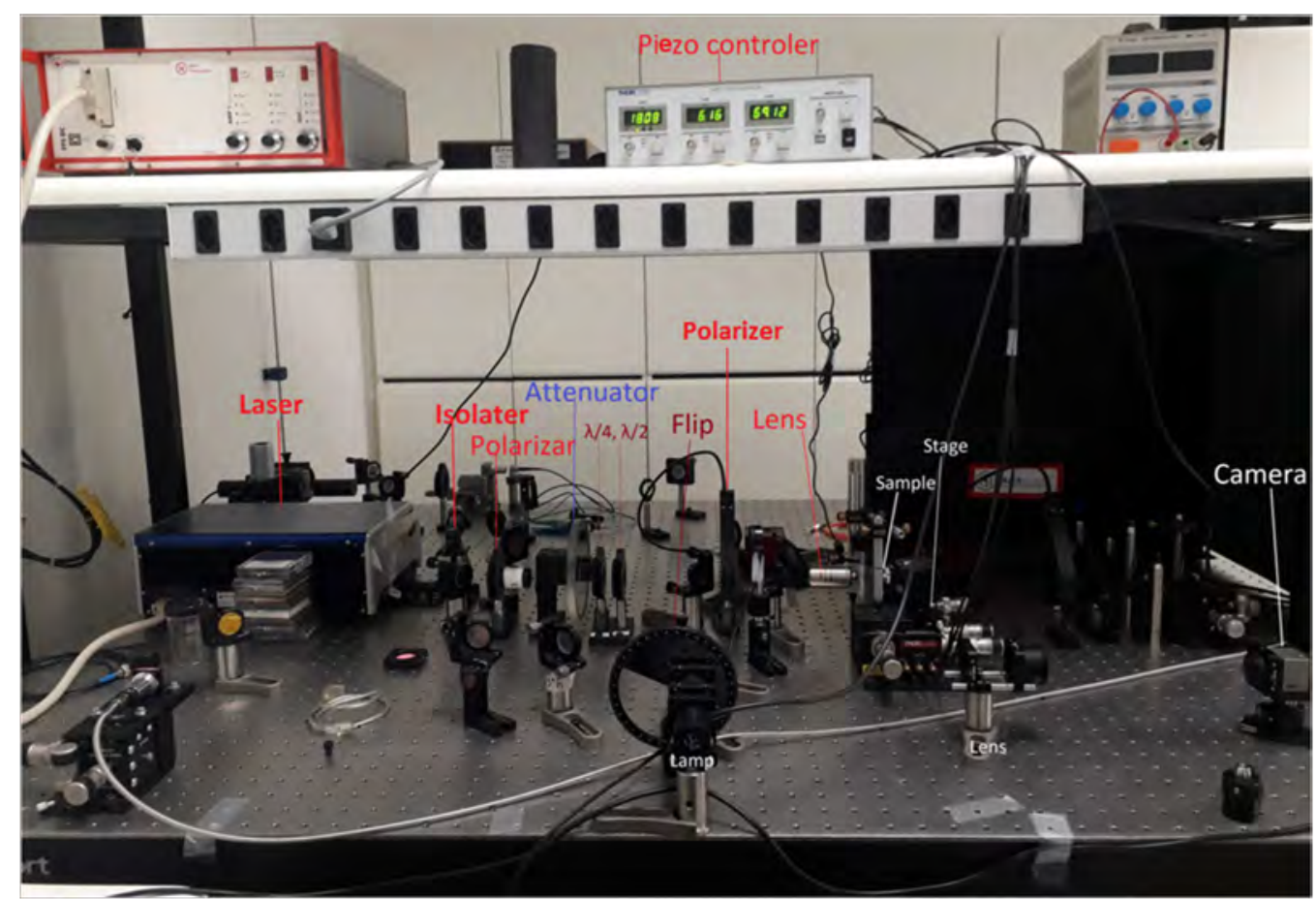

Figure 3.12: (a) Schematic diagram of harmonic generation (SHG and THG) microscopy system. (b) Experimental setup for harmonic generation measurements at Mackgraphe, Mackenzie Presbiterian University, SP. 
$50 \% \mathrm{~T} / \mathrm{R}$ at $950 \mathrm{~nm}$, DMLP950) being reflected in a moving mirror and then goes to the spectrometer (Andor Kymera spectrometer coupled to an iDus 416 Silicon CCD camera). A variable optical attenuator was used to control the average input power at $10 \mathrm{~mW}$ (measured after the linear polarizer). The power limitation is due to the integrity of the sample since higher powers could damage the $2 \mathrm{D}$ crystals. The second branch contains a microscope setup, which uses a red LED illumination source, a CCD camera, and other optical components, allowing the samples' real-time imaging.

THG signal (at $520 \mathrm{~nm}$ ) was also produced simultaneous with SHG signals, it accompanied of microscope setup was used to place the laser pump in the desired region onto the sample, and the collection of both by the spectrometer was performed separately due to the need to select the spectral region of interest before the collection.

For polarization dependence measurements, a quarter-wave plate and a linear polarizer in a rotation stage were used to rotate the light, leading to minimal intensity changes (less than $2 \%$ of variation in average power). The linear polarizer also operated as an analyzer. We used an electric piezo stage to ensure that all analyzed regions of the samples were in the same conditions. The optical axis was scanned to obtain the highest SHG intensity.

\section{9}

\section{Device Fabrication}

After a series of materials characterizations, high-quality samples are chosen to fabricate optoelectronic devices. To do it so, device fabrication process is followed. This device fabrication process was done in LabNano ate the Brazilian Center of Physical Research (CBPF - Centro Brasileiro de Pesquisas Físicas). To fabricate a device the following steps are defined.

- resist coating

- lithography

- development

- metal deposition

- lift off 


\subsection{1}

\section{Resist Coating}

The first step is to clean the surface of the sample to remove all contaminants in order to perform the lithography process. Use alcohol, water, and nitrogen blow to clean the surface. Avoid cleaning with acetone if you are fabricating device on the as-grown samples, acetone can destroy the thin TMD layer on the substrate. Spin coater shown in figure 3.13(a) is used to cover the sample with a thin polymer layer. The polymer resist might be different depending on the lithography we use. There are two main types of resists the positive and the negative resists. The suitable resist can be chosen by dose test. Drop a few drops of the polymer resist on the sample, in our case we choose AZ-1505 positive resist. Spin coat the resist at $4000 \mathrm{rpm}$ for $60 \mathrm{sec}$ this will gives us the $\sim 1 \mu \mathrm{m}$ thick polymer layer. The thickness of the polymer layer can be controlled either by the spinning velocity or the viscosity of the resist. The polymer is then hardened by baking it at $105^{\circ} \mathrm{C}$ for $50 \mathrm{sec}$ on a hot plate see figure 3.13(b). This polymer layer monomerizes when exposing to light.

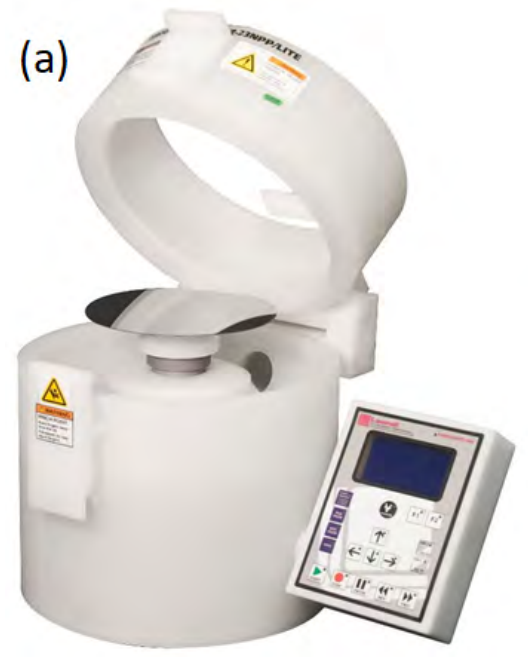

(b)

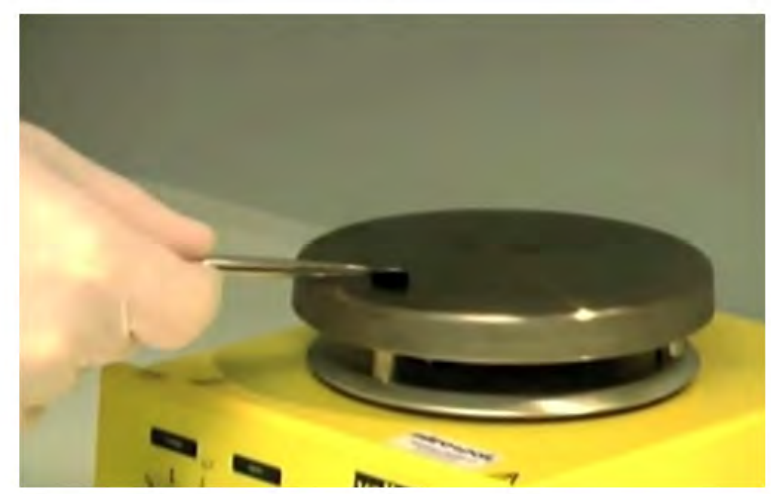

Figure 3.13: (a) Spin coater (model WS-650Hzb-23NPPB-UD-3, at labnano CBPF) was used to cover our sample with a resist. (b) Heating the sample coated with resist on a hot plate.

\subsection{2}

\section{Lithography}

Lithography is a technique of scanning a focused and controlled beam of electrons/Laser to draw a custom pattern on desired sample locations. There are two main types of lithography electron beam and photo-lithography. Both e-beam and photo-lithography have the same process steps in the flow. They 
have some differences in the specific photoresists, developers, and facilities. The e-beam lithography can draw patterns up to 4-10 nm [155] however, in photo-lithography we cannot go to this range. In our TMDs samples, we needed two/four-probe devices with minimum contact width of $10-20 \mu \mathrm{m}$ so photolithography was enough for us. We have used the Laser writer (Heidelberg $\mu \mathrm{PG}$ 101 Laser pattern generator) to draw the device circuit see figure 3.14. This Laser writer uses a focused high power laser $(633 \mathrm{~nm})$ to draw contact pattern on the surface of a substrate and has a critical dimension resolution of $\sim 3 \mu \mathrm{m}$. The designing procedure takes place in atmospheric pressure and humidity less than $60 \%$. The design pattern is controlled by LibreCAD software. A design pattern on LibreCAD is shown in figure 3.15(a), the LW simply copies the design pattern on the targeted area.

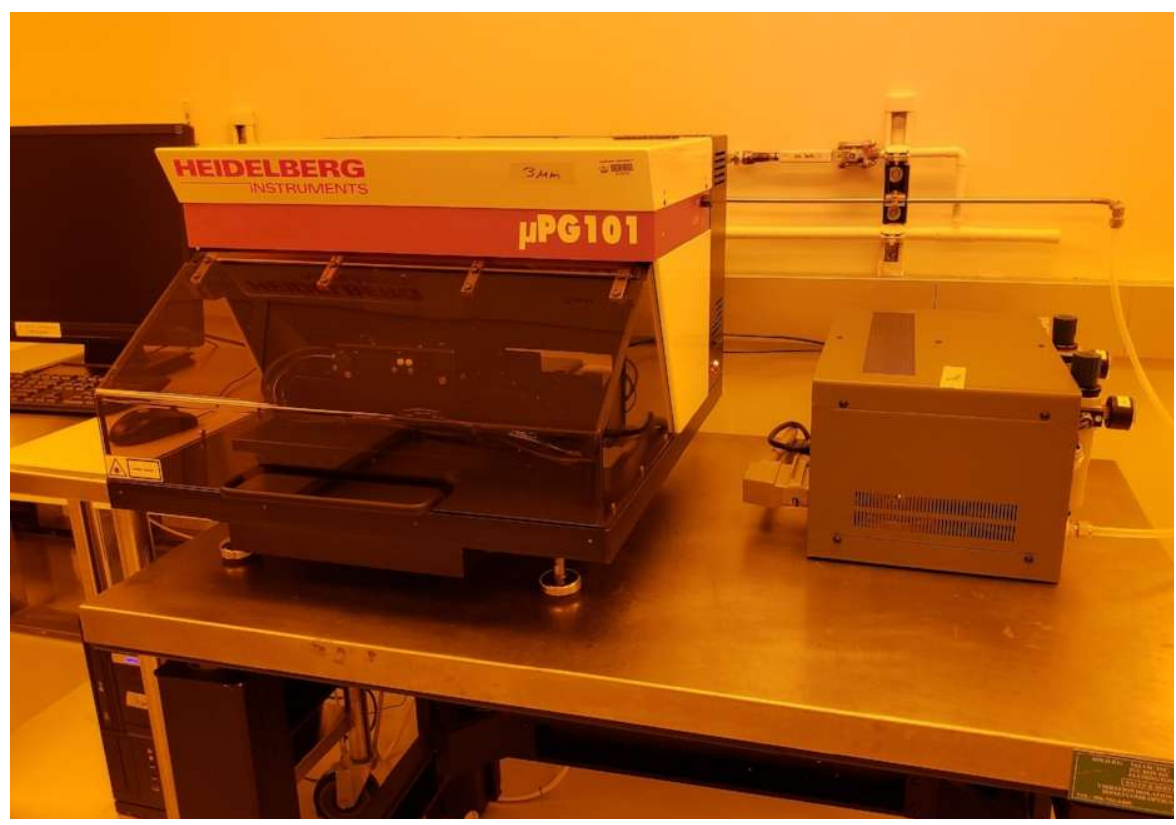

Figure 3.14: Laser writer lithography system model Heidelberg $\mu$ PG 101 Laser writer at lab nano $\mathrm{CBPF}$.

We need to fabricate the device on a TMD triangle located at a specific area on the substrate. Hence, before the lithography process, the exact distance was calculated from the substrate corners to the triangle with the optical microscope. Which made it easy to reach the targeted area in the Laser writer,s optical system. Now, suitable laser power (dose) must be chosen to expose the photoresist from the targeted area. If the device is to be made on transferred samples on $\mathrm{SiO}_{2} / \mathrm{Si}$ substrate, normal dose followed for $\mathrm{Si}$ substrate i.e. $5 \mathrm{~mW}$ $70 \%$ is good enough. But for the as grown samples, the dose is different and depends on the temperature and time of growth of the sample. A dose test is recommended for every sample, otherwise, the laser will not completely remove 
the photoresist from the sample. The best way is to cut a piece from the sample and expose it with different doses. For example, a dose test performed on a sample is shown in figure $3.15(\mathrm{~b})$, showing $5 \mathrm{~mW} 85 \%$ is the best dose for this sample.

(a)

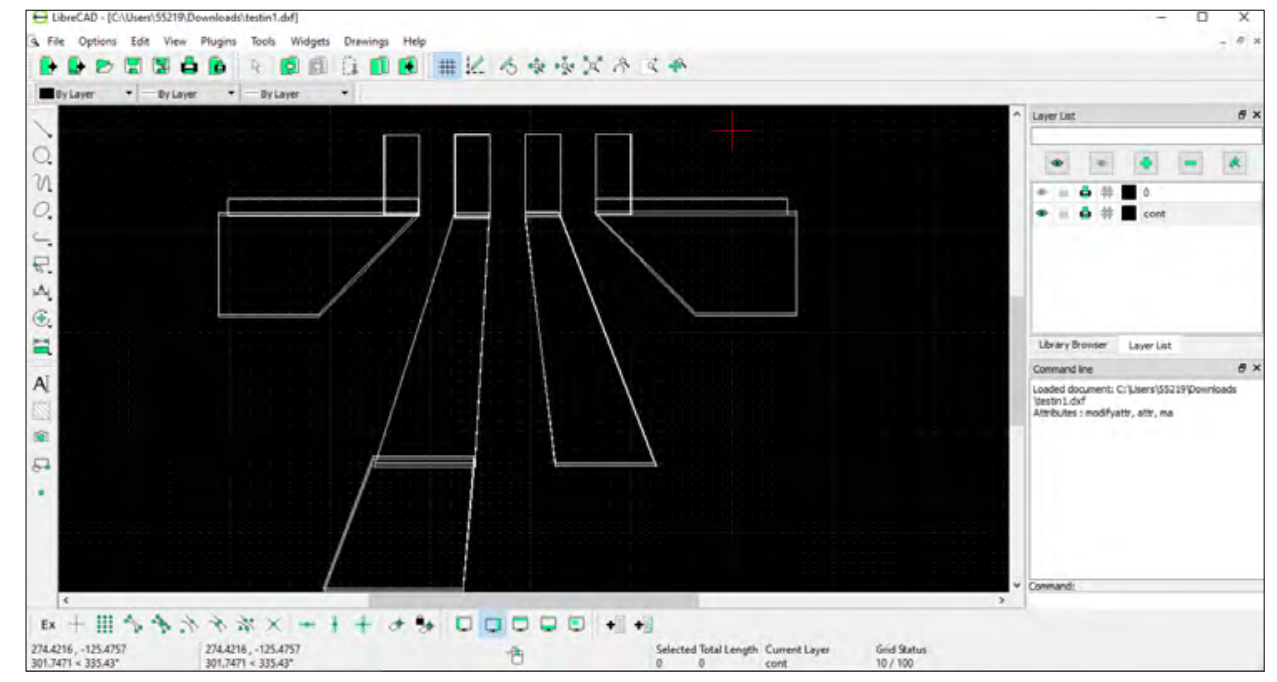

(b)
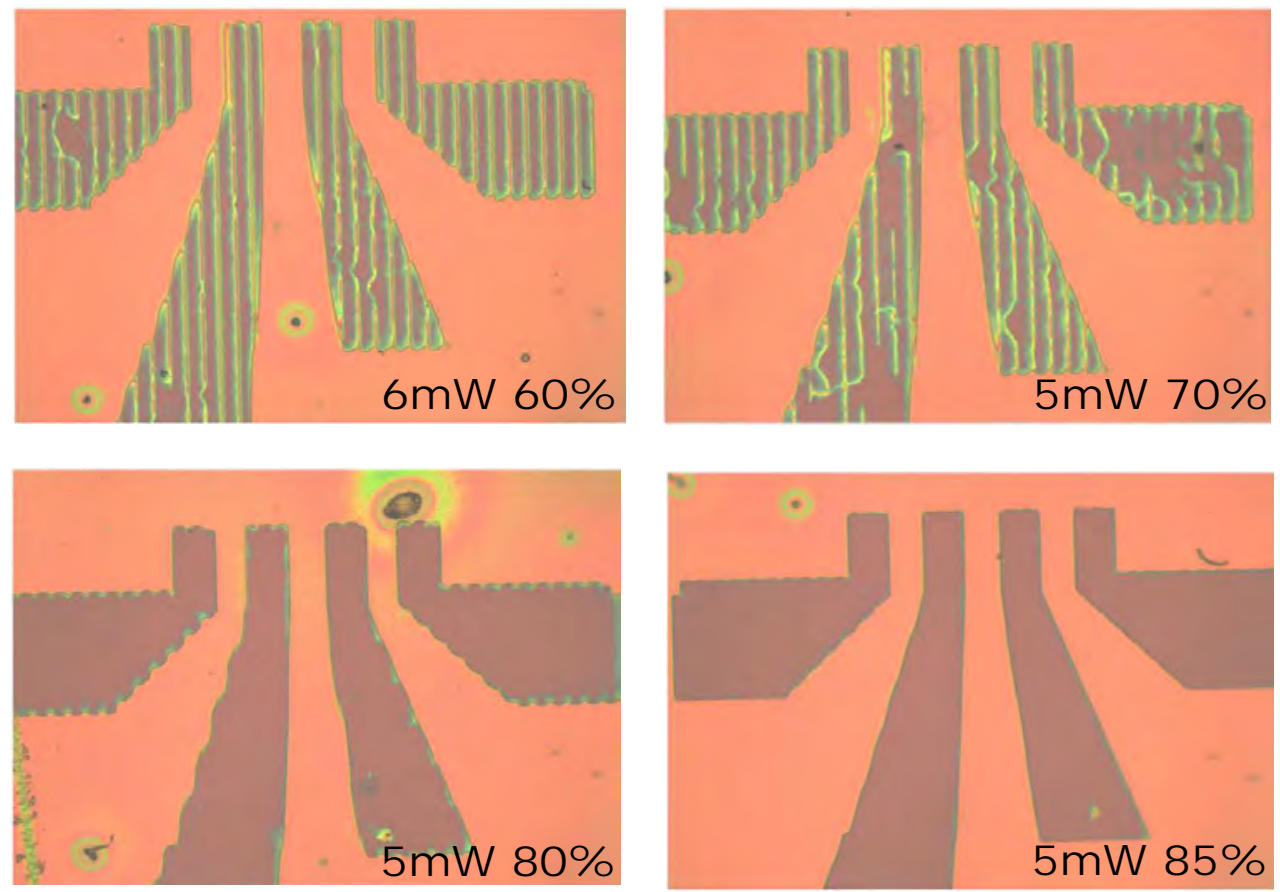

Figure 3.15: (a) Design on a LibreCAD program. (b) Optical images of a sample after development on which dose test was performed with different laser powers. 


\subsection{3}

\section{Development}

After lithography, the exposed sample is taken to the development stage. During development, a specific chemical solution (depending on the resist we have used before) is applied to the surface causing photoresist reaction. The molecules in the resist which are not subjected to the laser are strongly bonded together in long chains, while the area which is exposed by the laser is monomerized. After the development process, the non-polymerized parts of the resist decompose leaving the polymerized resist only. In our case we are using AZ-1505 photoreist then we need AZ 300 MIF developer. The sample should be immediately taken out from the LW after the exposure. Hold the sample in your hand dip it in the developer and move it slowly in the liquid for $60 \mathrm{sec}$. After $60 \mathrm{sec}$ suddenly dip the sample in distilled $\mathrm{H}_{2} \mathrm{O}$ and do the same for $60 \mathrm{sec}$ in $\mathrm{H}_{2} \mathrm{O}$, finally dry your sample with air blow. This will dissolve the photoresist which was exposed to laser beam while on the other area resist will be hardened by the developer.

\subsection{4}

\section{Metal deposition}

The next step is the deposition of metal over a design pattern. The metal can be deposited by a sputtering or evaporator system. We have used Edwards thermal evaporator equipment to deposit copper on our sample. First, the sample was placed inside the chamber and vacuum was created to $\sim 2 \times 10^{-6}$ torr. The copper metal is placed in a molybdenum boat through which $\sim 12 \mathrm{~A}$ current is passed. Then the metal particles are ejected by thermally heating of copper target. That deposits metal over the surface of our sample. On the part where the circuit pattern was drawn, metal can stick directly to the exposed sample. While everywhere else there is still photoresist between the metal and the sample surface. The deposited metal film thickness was determined by a $6 \mathrm{MHz}$ quartz crystal micro-balance assisted by the FTM6 Edwards control unit. Figure 3.16 shows thermal evaporator equipment. 


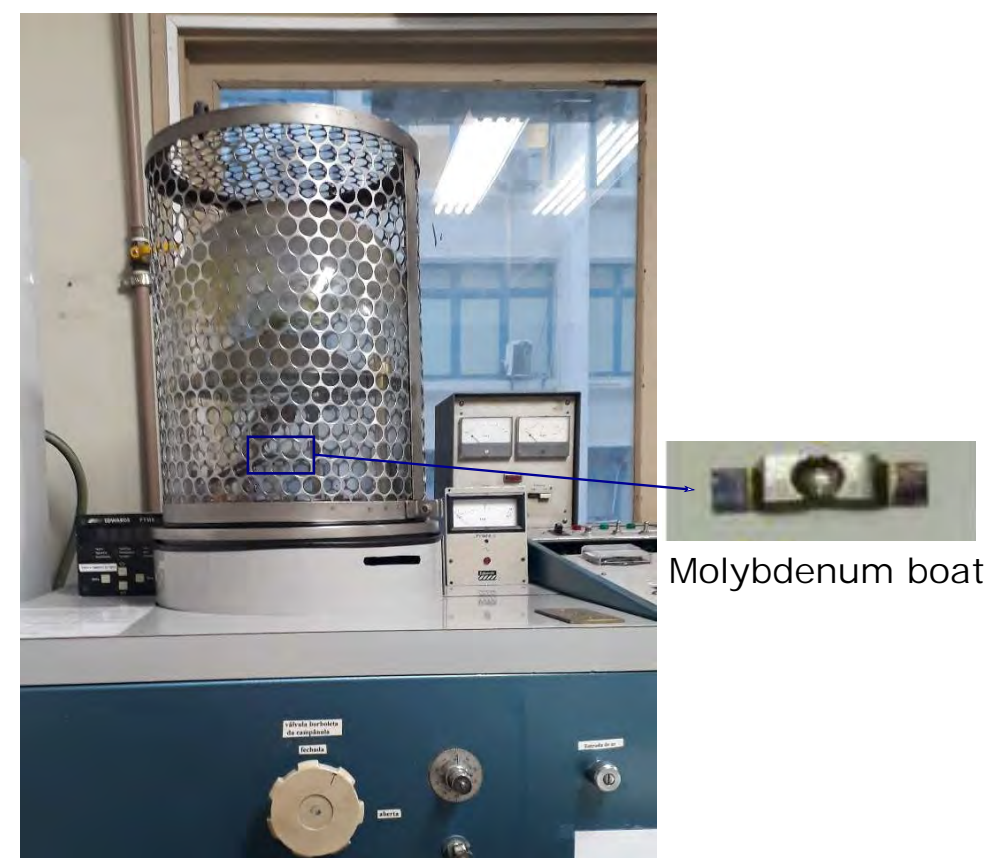

Figure 3.16: Edwards thermal evaporator equipment at vandegraff PUC-Rio.

\section{9 .5}

\section{Lift off}

The final step is the removal of the photoresist and unwanted metal. Silicon substrates are usually treated by plasma etching after the step of photolithography to remove the photoresist residual and clean the Si surface for good device performance. However, if plasma etching is used to treat and clean the surface of a TMDs, they will be damaged severely or etched away completely because of their atomically thin layered structure. An alternative and feasible processing method is to dissolve the photoresist in acetone. This process has to be carried out very carefully and slowly, to avoid damaging circuit pattern or sample during lift off. In my personal experience, this process should be done under an optical microscope. Put your sample in a glass container, focus microscope on the contacts. Now put acetone in the glass container, acetone quickly starts dissolving the photoresist and metal on the resist will also start peeling off. When you see the metal is completely removed especially at the area where contact pattern was drawn on a TMD triangle. Take out the sample immediately and wash it with distilled $\mathrm{H}_{2} \mathrm{O}$. Normally this will take 2-3 min but also depends on the thickness of the deposited metal and photoresist. Dissolving sample for long in acetone will result in damaging the TMD triangle, and dissolving for less time the metal may not be removed completely and we have a short circuit in the device. That's why this process should be done under a microscope. Flow chart of the whole process is shown in figure 3.17. After metal lift-off, a bottom-gated FET device is ready. 


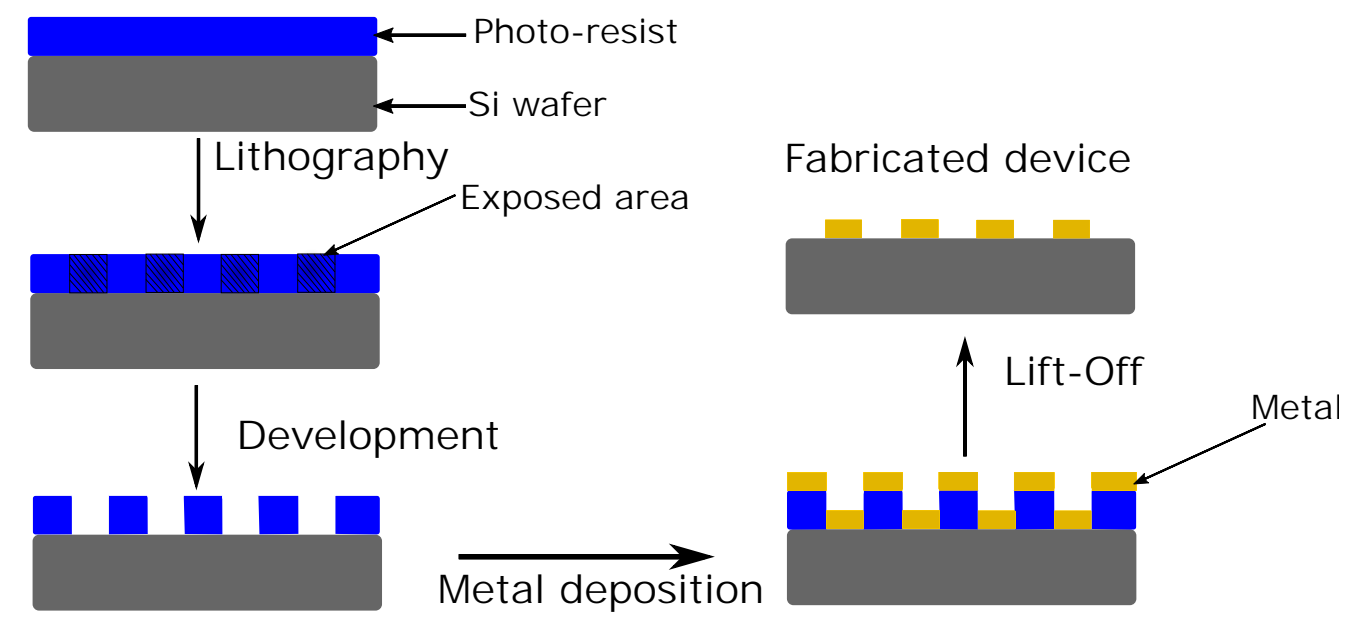

Figure 3.17: General device fabrication procedure.

\subsection{0}

\section{Electrical Measurements}

After fabricating the devices, the electrical performance of the device was measured at room temperature under ambient conditions. The output curve $\left(\mathrm{I}_{D S}\right.$ vs. $\left.\mathrm{V}_{D S}\right)$ was measured by applying drain-source voltage from Keithley SMU 2400 (with a current source and integrated digital multi-meter). The $\left(\mathrm{I}_{D S}\right.$ vs. $\left.\mathrm{V}_{D S}\right)$ graphs were plotted using a Kickstart 2.1.1 software. The transfer curve was measured by manually applying back gate voltage from p-doped silicon substrate. Figure 3.18 shows the instrumentation used in the experiments.

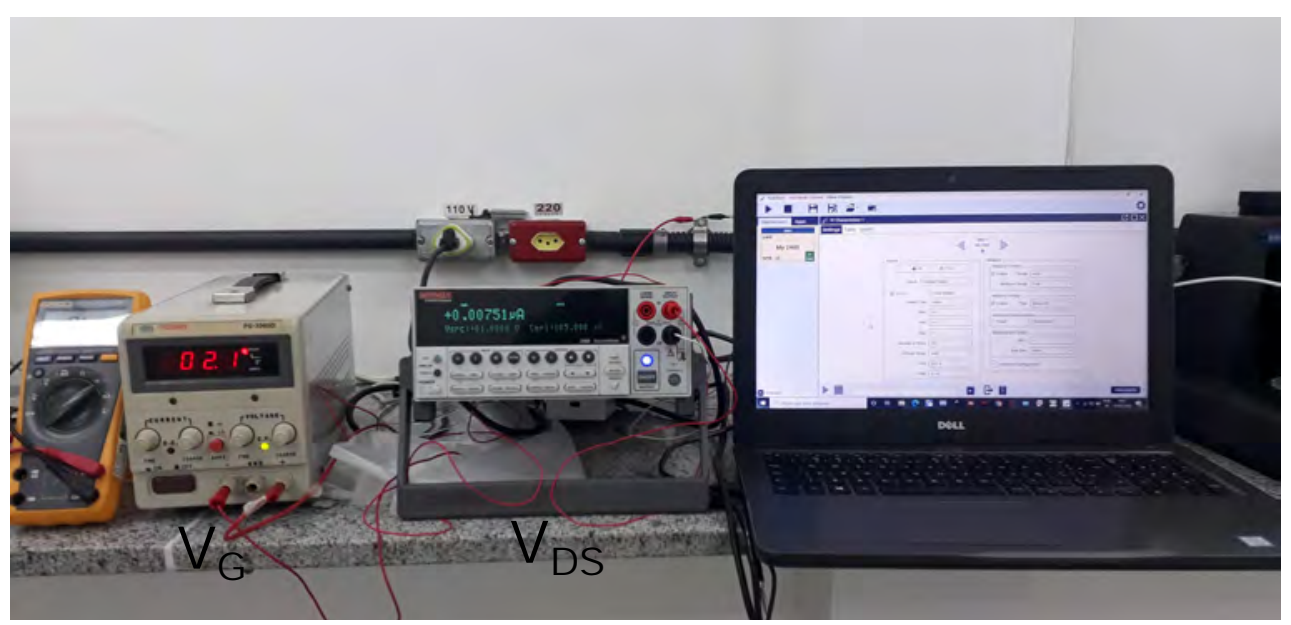

Figure 3.18: Experimental setup for transport measurements. 


\subsection{0 .1}

\section{Electro-Raman}

The electro-Raman measurements were performed by applying voltage to our device and at the same time taking Raman spectra. Figure 3.19 shows a schematic of the experimental setup of the spectroscopy measurement with electronics. A confocal Raman spectroscopy system (NT-MDT, NTEGRA spectrometer) was used with the excitation laser of $473 \mathrm{~nm}$. The devices were on a Si substrate, which was mounted on a homemade electronic board. A gate voltage is applied by a voltage source. This is actually a combination of two setups see figure 3.19, a Raman spectrometer working separately, and voltage is applied to the sample only.

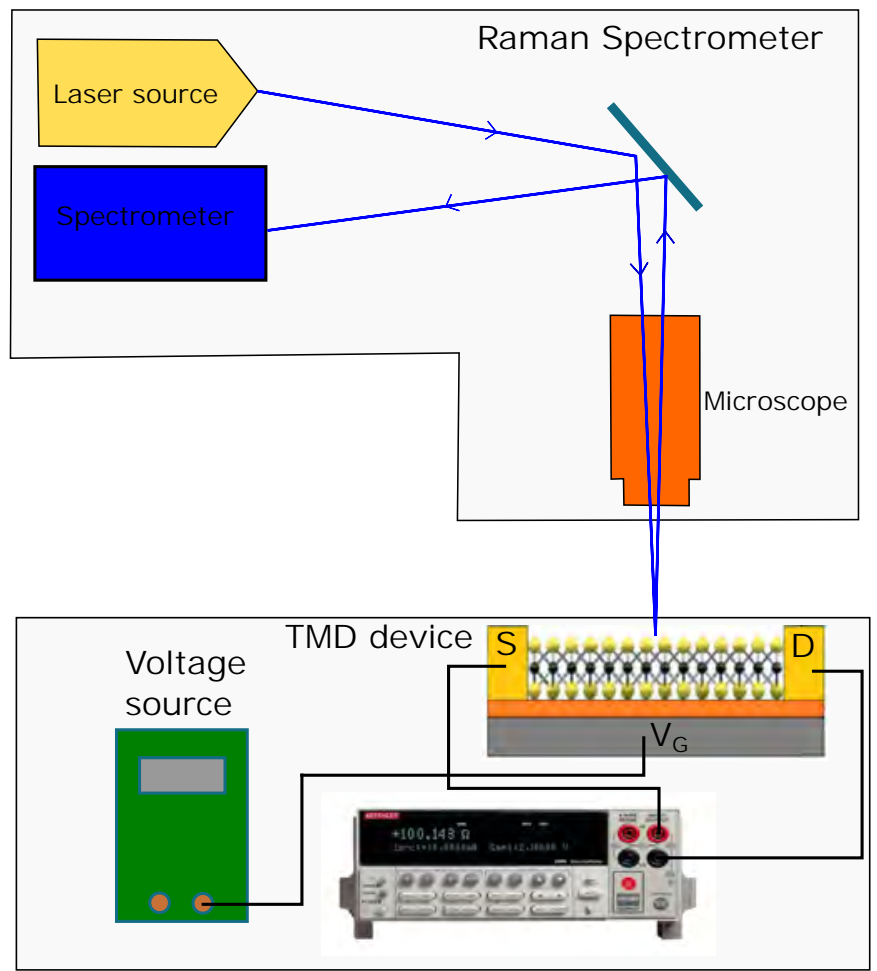

Figure 3.19: Schematic of the experimental setup. 


\section{4 \\ CVD Grown $\mathrm{MoS}_{2}, \mathrm{WS}_{2}$ Monolayer, and their Vertical Het- erostructure}

In this chapter, the results of the CVD grown monolayer $\mathrm{MoS}_{2}, \mathrm{WS}_{2}$, and their vertical heterostructure are presented. Their characterizations by atomic force microscopy, Raman and PL spectroscopies are discussed. Detailed explanation of sodium mediated low temperature synthesis of monolayer molybdenum disulfide single crystal triangles and polycrystalline film. The catalytic behavior of sodium in decreasing the synthesis temperature is explained by DFT calculations.

\section{1}

\section{Introduction}

TMDs monolayers have been successfully synthesized by atmospheric pressure chemical vapor deposition (APCVD) on $\mathrm{SiO}_{2} / \mathrm{Si}$ substrate. The APCVD process is explained in detail (see chapter 3, section 3.1). After the successful growth of TMDs, several characterization techniques were used to examine the surface morphology, thickness, and optical quality of our samples. The most straightforward technique is the optical microscopy. Layers of the TMDs can be distinguished by colour contrast on $\mathrm{SiO}_{2}$ substrate arising from the effect of light interference [156-158]. Thus, optical microscopy is usually first used to inspect the TMDs, to get a rough idea of the sample morphology and size before more accurate and higher resolution techniques are conducted. The monolayer thickness and sample quality were also confirmed by three more characterizations AFM, Raman, and PL spectroscopies.

\section{2}

\section{$\mathrm{MoS}_{2}$ and $\mathrm{WS}_{2}$ Monolayer}

Typical images acquired by optical microscopy of $\mathrm{MoS}_{2}$ and $\mathrm{WS}_{2}$ monolayers grown on $\mathrm{SiO}_{2} / \mathrm{Si}$ substrates at high temperature are shown in figure 4.1(a,b). The difference in colour contrast arises due to the different thicknesses of the oxide layer on the Si substrate. The surfaces are covered with an abundance of randomly oriented equilateral triangles typical of growth on the $\mathrm{SiO}_{2} / \mathrm{Si}$ substrate $[15,159]$. Furthermore, the grain-boundaries can be observed 


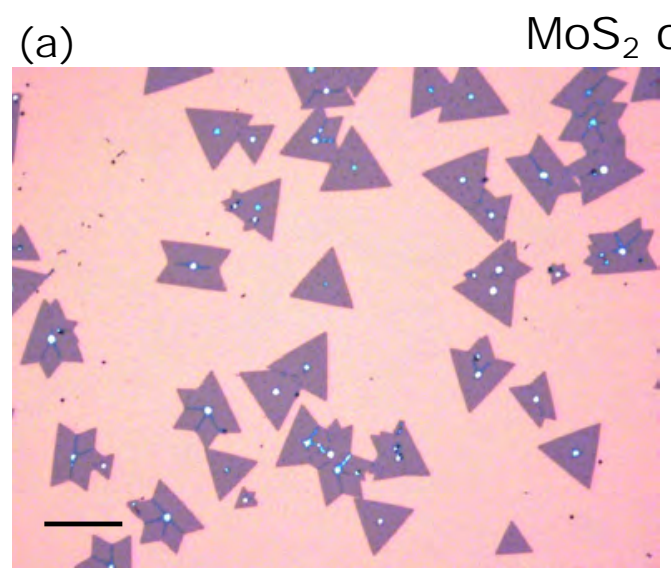

$\mathrm{Si} / \mathrm{SiO}_{2}$

(b)

$\mathrm{WS}_{2}$ on $\mathrm{Si} / \mathrm{SiO}_{2}$
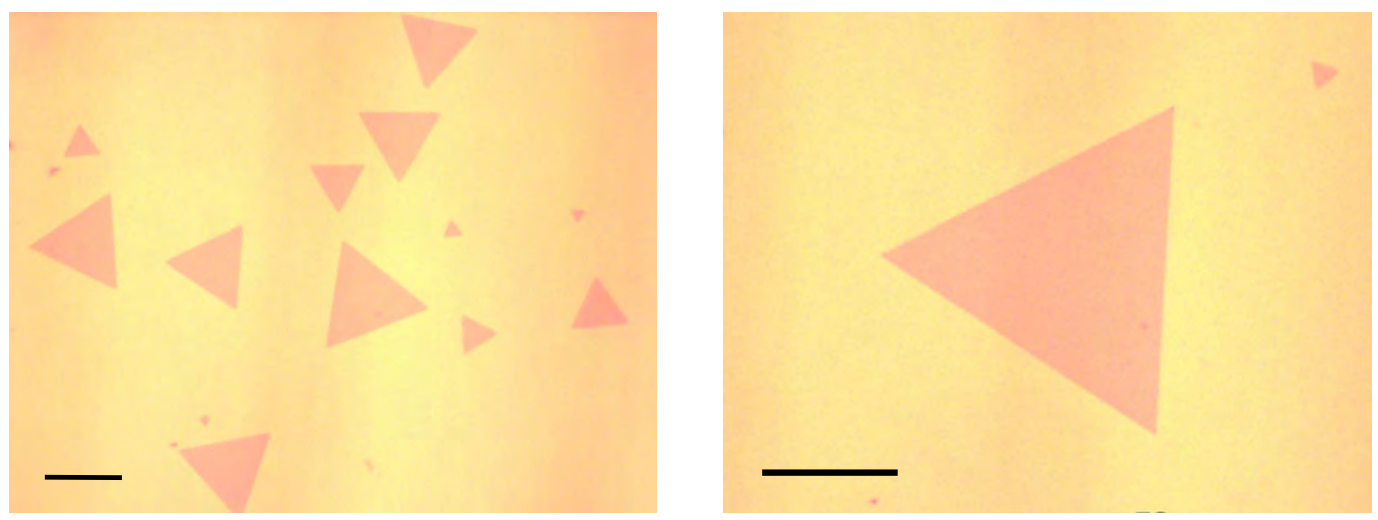

Figure 4.1: (a) Optical microscopy images of $\mathrm{MoS}_{2}$ grown on $\mathrm{SiO}_{2} / \mathrm{Si} 285 \mathrm{~nm}$, scale bars are $50 \mu \mathrm{m}$ and $20 \mu \mathrm{m}$. (b) Optical images of $\mathrm{WS}_{2}$ grown on $\mathrm{SiO}_{2} / \mathrm{Si}$ $400 \mathrm{~nm}$.

caused by the coalition of adjacent triangles which is inevitable in CVD grown TMDs [67]. The size of the triangles ranges 20 - $40 \mu \mathrm{m}$. A small dot at the centre of the $\mathrm{MoS}_{2}$ triangles is the nucleation sites and the dark spots are bilayer areas on $\mathrm{MoS}_{2}$ triangles. The growth occurs at a single nucleation point leading to a monolayer triangular island that grows outward. However, the $\mathrm{WS}_{2}$ triangles are very clean and in perfect shape showing no dirt or bilayer region on the entire triangular crystallite see figure 4.1(b). Just as our observation, $\mathrm{WS}_{2}$ was reported to be grown much cleaner and in perfect shaped in CVD synthesis compared to $\mathrm{MoS}_{2}[34,160]$, because in sulphur rich atmosphere it common to have an extra layer of $\mathrm{S}$ over $\mathrm{MoS}_{2}$ layer. It can be noted that the colour contrast of all the $\mathrm{WS}_{2}$ triangles regardless of their size is uniform, which indicates they all are monolayer [157].

To identify the height topography of these triangular islands, atomic force microscopy measurements were carried out. The AFM image of one $\mathrm{MoS}_{2}$ triangle is shown in figure 4.2(a). In the image we can see the colour contrast between the substrate and the triangle, the line profile inset is showing the 
(a)

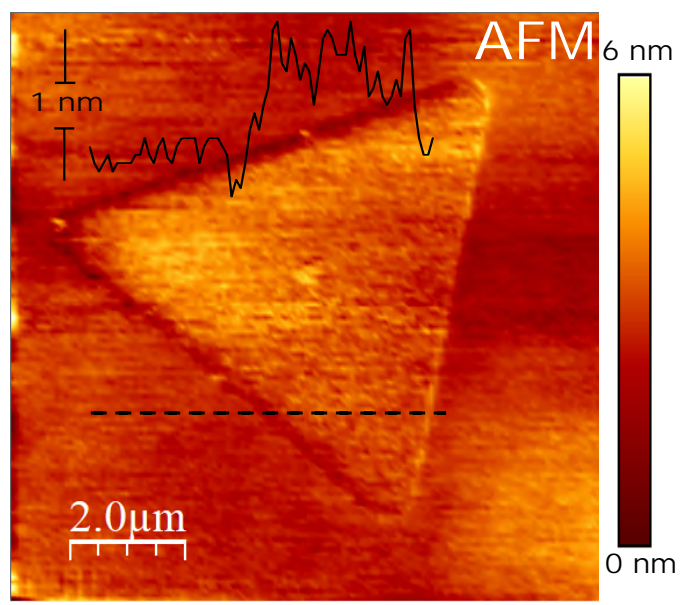

(b)

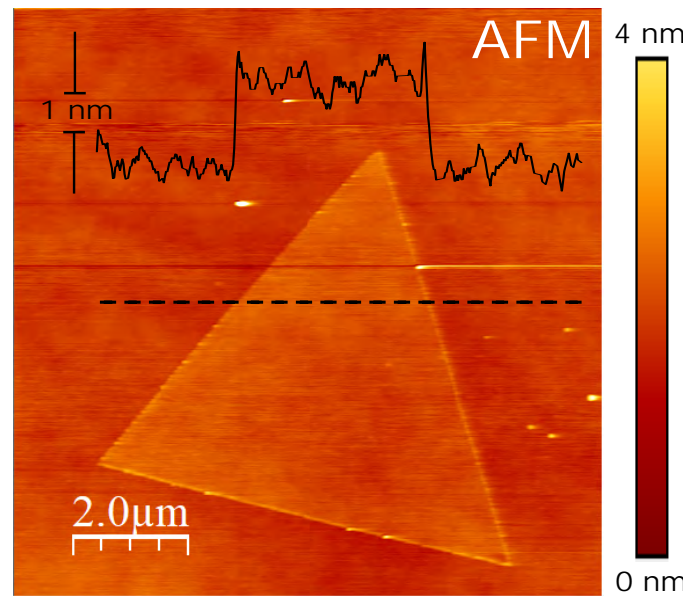

Figure 4.2: (a) AFM topography, profile of $\mathrm{MoS}_{2}$ flake, inset the line profile shows the step of $1 \mathrm{~nm}$. (b) AFM image of $\mathrm{WS}_{2}$ flake.

height which is $\sim 1 \mathrm{~nm}$. Figure $4.2(\mathrm{~b})$ shows the AFM image of a $\mathrm{WS}_{2}$ triangle. The height profile in the inset is $\sim 0.9 \mathrm{~nm}$ which confirms the monolayer thickness [160]. The homogeneous colour contrast in $\mathrm{WS}_{2}$ crystal shows, it is much cleaner and has smoother surface compared to $\mathrm{MoS}_{2}$ which can also be noticed in the optical images.

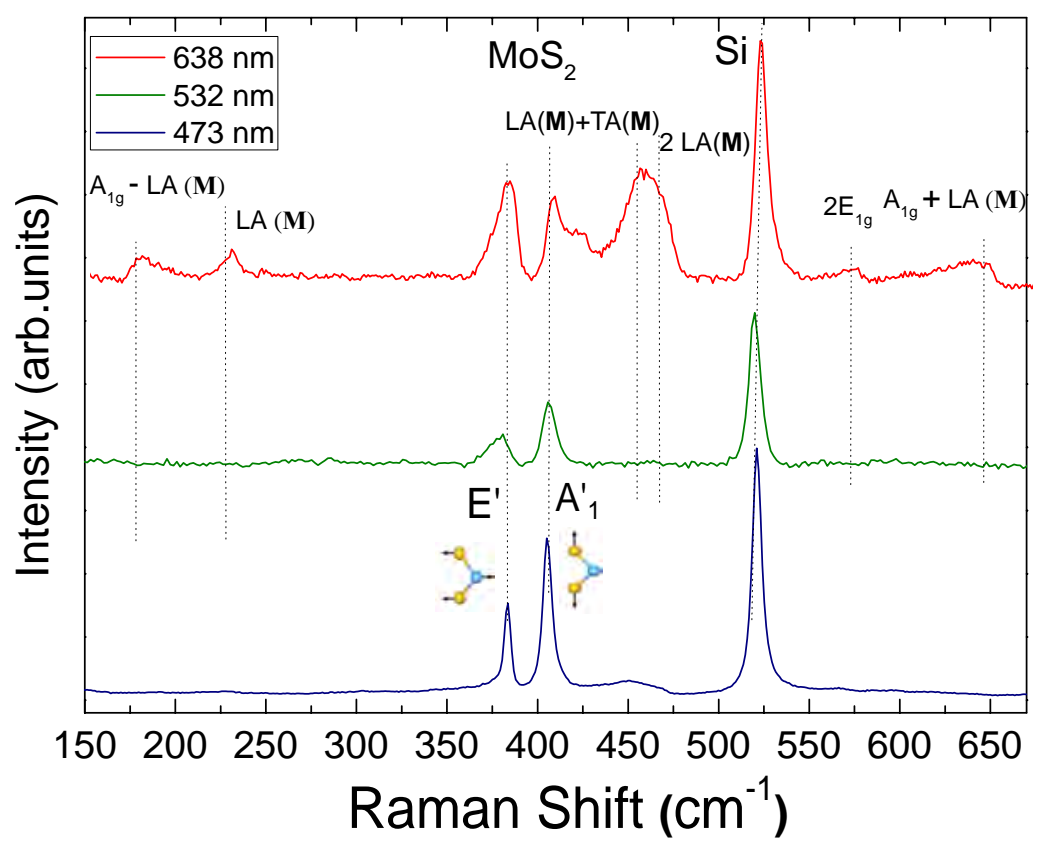

Figure 4.3: Raman spectra of $\mathrm{MoS}_{2}$ at different excitation wavelengths.

To study the vibrational modes of our samples Raman spectroscopy was used at room temperature under ambient conditions. Raman spectra also provide additional confirmation of the monolayer nature and an indication of 
sample quality. Representative Raman spectra obtained from three different excitation wavelengths $(473,532,638 \mathrm{~nm})$ for monolayer $\mathrm{MoS}_{2}$ triangle is shown in figure 4.3. For $638 \mathrm{~nm}$ laser, we observe many peaks because this wavelength is in resonance with the $\mathrm{A}$ exciton of $\mathrm{MoS}_{2}$. Except the two peaks associated to first-order $\mathrm{E}^{\prime}$ and $\mathrm{A}_{1}^{\prime}$ modes, all the other features are contributions from different resonant Raman process.

Here we observe an $\mathrm{A}_{1 g^{-}} \mathrm{LA}(\mathbf{M})$ mode at $179 \mathrm{~cm}^{-1}$ which is assigned as two-phonon difference combination mode [161]. Another peak at $228 \mathrm{~cm}^{-1}$ is LA mode near $\mathbf{M}$ point at Brillouin zone, and therefore, it is not a first-order Raman peak. The LA(M) mode is originated from a double resonance process involving one inelastic phonon scattering and one elastic defect scattering. This mode can only be active due to defects in the crystal lattice $[46,162]$. The intensity of $\mathrm{LA}(\mathbf{M})$ mode is directly proportional to the average of interdefect distance [46]. A prominent appearance of this mode indicates the structural defects in the crystal $[163,164]$. The peak at $423 \mathrm{~cm}^{-1}$ is referred to $\mathrm{LA}(\mathbf{M})+\mathrm{TA}(\mathbf{M})[165]$. Here we get $2 \mathrm{LA}$ band $460 \mathrm{~cm}^{-1}$ which is the strongest feature in the second-order spectrum. The high energy component of the peak at $465 \mathrm{~cm}^{-1}$ was usually related to $2 \mathrm{LA}$ at the $\mathbf{M}$ point of the $\mathrm{MoS}_{2}$ Brillouin zone. In high wavenumber region, we observe two prominent peaks at $572 \mathrm{~cm}^{-1}$ and $641 \mathrm{~cm}^{-1}$ which we referred to $2 \mathrm{E}_{1 g}$ and $\mathrm{A}_{1 g}+\mathrm{LA}(\mathbf{M})$ modes [99]. The most prominent modes in all the spectra are the first order in-plane phonon mode $\mathrm{E}^{\prime}$ and the out-of-plane $\mathrm{A}_{1}^{\prime}$ mode. These modes were observed at $383.7 \mathrm{~cm}^{-1}$, $403.8 \mathrm{~cm}^{-1}$. The difference in peak position between these modes is $20.1 \mathrm{~cm}^{-1}$ correspondent to monolayer [166].

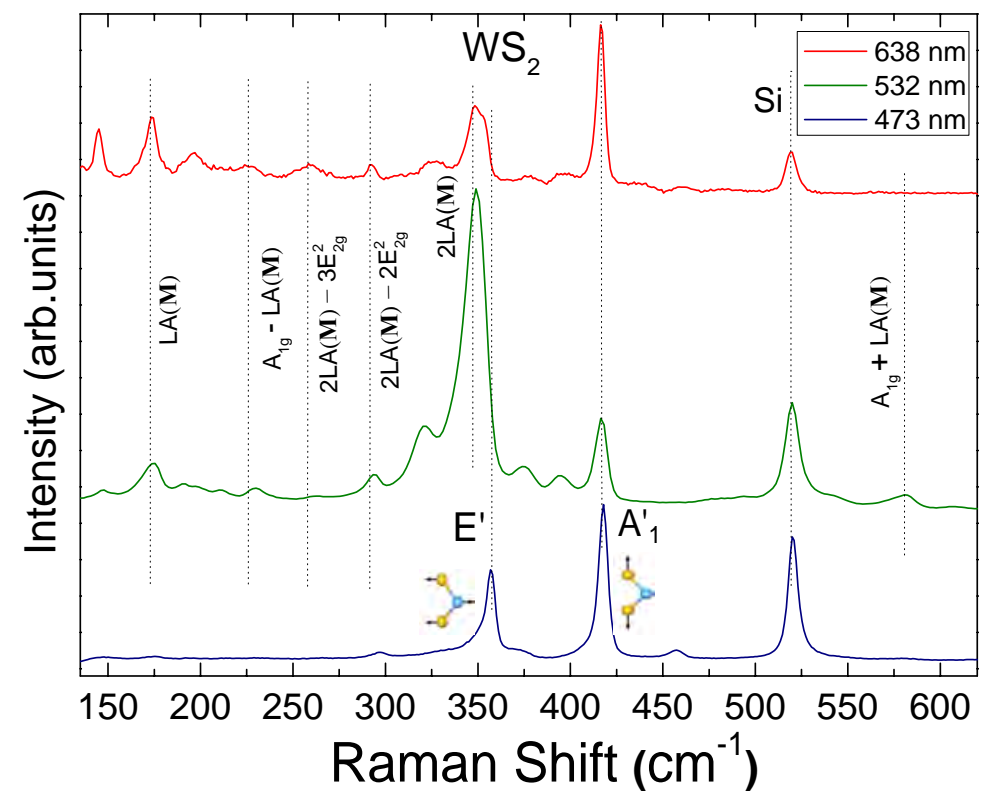

Figure 4.4: Raman spectra of $\mathrm{WS}_{2}$ at different excitation wavelengths. 
The Raman spectra from excitation wavelengths $(473,532,638 \mathrm{~nm})$ of $\mathrm{WS}_{2}$ sample are shown in figure 4.4. The $532 \mathrm{~nm}$ laser excitation generates a double-resonant Raman process involving the LA(M) mode. The peak at $145 \mathrm{~cm}^{-1}$ is related to out-of-plane acoustic phonons from the border of the BZ, namely ZA(M) [167]. Peak $176 \mathrm{~cm}^{-1}$ can be attributed to a longitudinal acoustic mode from the M point of the BZ, LA(M). All the other second order peaks were assigned in accordance to references $[45,168,169]$. The first order $\mathrm{E}^{\prime}$ mode is usually overlapped by the $2 \mathrm{LA}(\mathbf{M})$ mode in the Raman spectra under resonant condition. To get the exact information about $\mathrm{E}^{\prime}$ and $\mathrm{A}_{1}^{\prime}$ modes, the $473 \mathrm{~nm}$ excitation is used to obtain the non-resonant Raman signal of the $\mathrm{WS}_{2}$ sample. We found the first order in plane $\mathrm{E}^{\prime}$ mode at $358.8 \mathrm{~cm}^{-1}$ and out of plane $\mathrm{A}_{1}^{\prime}$ mode at $419.9 \mathrm{~cm}^{-1}$. The difference between these two peaks is 61.1 $\mathrm{cm}^{-1}$ which corresponds to monolayer [45].

(a)

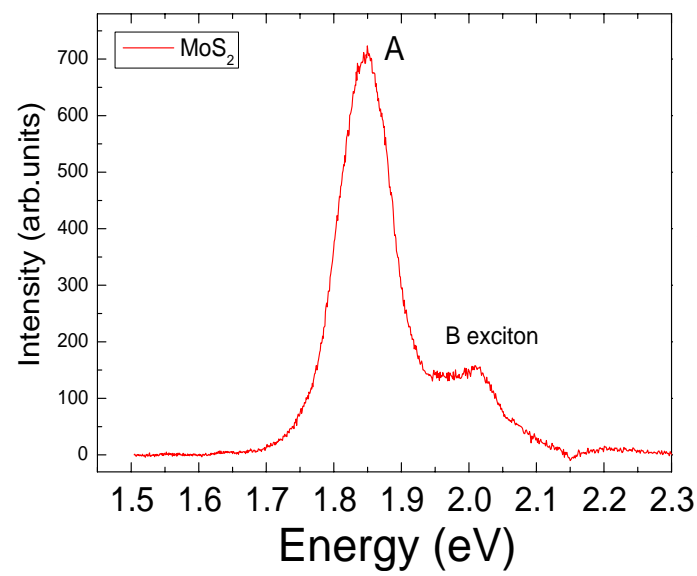

(b)

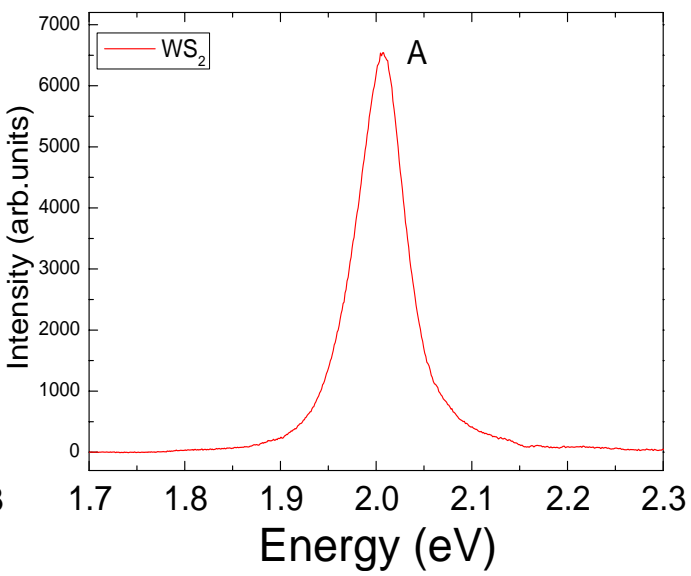

Figure 4.5: (a) Photoluminescence spectra of monolayer $\mathrm{MoS}_{2}$. (b) Photoluminescence spectra of monolayer $\mathrm{WS}_{2}$.

Figure 4.5(a) shows the PL spectrum of $\mathrm{MoS}_{2}$ triangle on $\mathrm{SiO}_{2} / \mathrm{Si}$ substrate, exhibited peaks located at $1.83 \mathrm{eV}$ and $2 \mathrm{eV}$. The two peaks in the PL spectrum are known as A and B excitons, are due to a strong excitonic effect in $\mathrm{MoS}_{2}[11,38]$. The separation of $(\sim 0.17 \mathrm{eV})$ between $\mathrm{A}$ and $\mathrm{B}$ excitons come from the spin-orbit splitting at the top of the valence band at the $\mathbf{K}$ point of the BZ, from $\mathrm{d}_{x^{2}-y^{2}}$ and $\mathrm{d}_{x y}$ states to $\mathrm{d}_{z^{2}}$ type states $[109,170,171]$.

The PL spectra of $\mathrm{WS}_{2}$ is given in figure 4.5(b). The PL shows an intense peak at $2.05 \mathrm{eV}$ [172]. Both $\mathrm{MoS}_{2}$ and $\mathrm{WS}_{2}$ show a sharp, and intense peak in a $\mathrm{PL}$ spectrum which indicates the direct bandgap normally observed in monolayer $[11,102,173]$. The PL emission efficiency of $\mathrm{WS}_{2}$ monolayer is more than 10 times higher than $\mathrm{MoS}_{2}$ monolayer. The difference between the valence band and conduction band energy of $\mathrm{WS}_{2}$ is higher than that of $\mathrm{MoS}_{2}$ [174]. 
Chapter 4. CVD Grown $\mathrm{MoS}_{2}, \mathrm{WS}_{2}$ Monolayer, and their Vertical

Hence, is the bandgap energy and $\mathrm{PL}$ too, so $\mathrm{WS}_{2}$ is superior for device applications.

\section{3}

\section{$\mathrm{MoS}_{2} / \mathrm{WS}_{2}$ Vertical Heterosctructure}

(a)

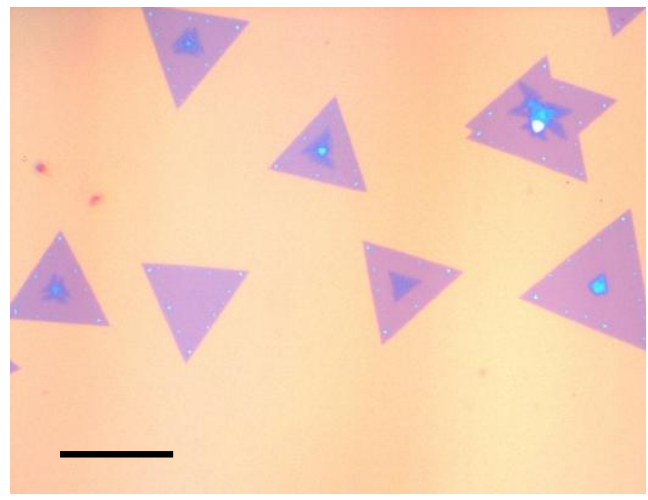

(c)

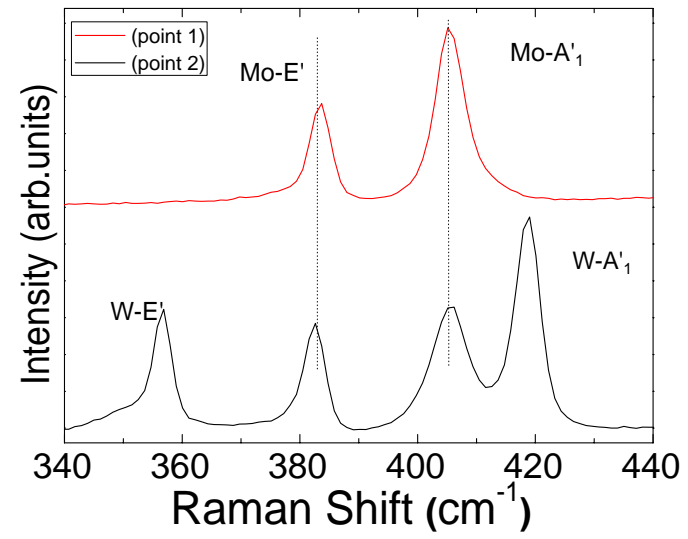

(b)

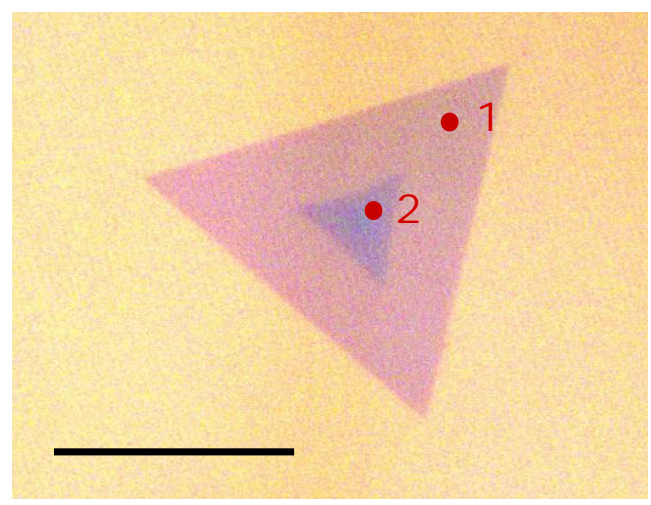

(d)

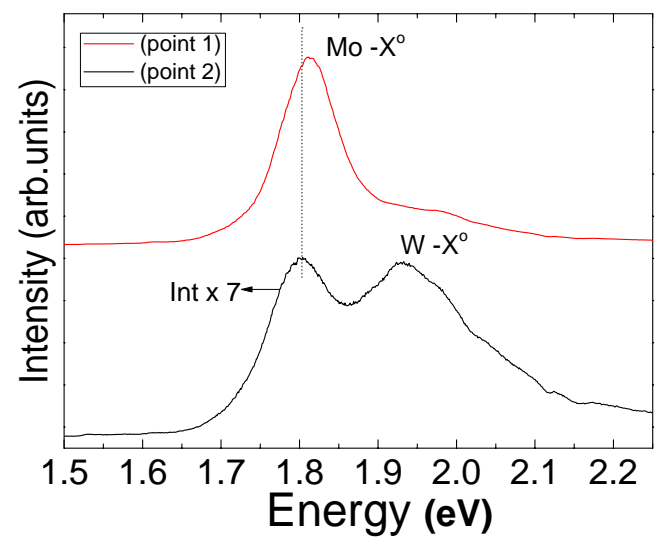

Figure 4.6: (a,b) Optical images of $\mathrm{MoS}_{2} / \mathrm{WS}_{2}$ vertical heterostructure scale bar is $20 \mu \mathrm{m}$. (c) Raman spectra of $\mathrm{MoS}_{2} / \mathrm{WS}_{2}$ heterostructure taken at point 1 and 2 as shown in figure 4.6(b). (d) Photoluminescence of the same triangle at point 1 and 2 .

We have grown $\mathrm{MoS}_{2} / \mathrm{WS}_{2}$ vertical heterostructures by APCVD. In these samples, we have a monolayer of $\mathrm{MoS}_{2}$ and right on top, a $\mathrm{WS}_{2}$ layer. The optical images of $\mathrm{MoS}_{2} / \mathrm{WS}_{2}$ heterostructure triangles are shown in figure 4.6(a,b). Not all the triangles are perfectly bilayer heterostructures, some have incomplete growth of the top layer, some have multiple layers on top see figure 4.6(a). The Raman spectra of $\mathrm{MoS}_{2} / \mathrm{WS}_{2}$ flake shown in figure 4.6(c), was taken at point 1 and 2 marked in figure 4.6(b). It is clear that we have only $\mathrm{MoS}_{2}$ at the corner of the triangle (point 1) and at the centre at (point 2) we observe four sharp peaks, the first order in-plane and out of plane vibrational 
modes from $\mathrm{WS}_{2}$ and $\mathrm{MoS}_{2}$ layers. The PL at these two points is also shown in figure 4.6(d). In the heterostructure region we saw two peaks corresponding to the excitonic transition energies of $1.81 \mathrm{eV}$ and $1.93 \mathrm{eV}$. The peaks can be attributed to $\mathrm{MoS}_{2}$ bottom and $\mathrm{WS}_{2}$ top layers. The PL intensity at (point 2) is multiplied by 7 to be compared with the top graph.

\section{4}

\section{Sodium Mediated Low Temperature Synthesis of Monolayers of Molyb- denum Disulfide}

We have discussed the high temperature (i.e $\geq 850^{\circ} \mathrm{C}$ ) synthesis of TMDs. The high temperatures of synthesis is a barrier to mass and low-cost production of monolayers TMDs. One route to reduce the fabrication cost is to decrease the synthesis temperature. Here, we report the CVD synthesis of $\mathrm{MoS}_{2}$ monolayer on $\mathrm{SiO}_{2} / \mathrm{Si}$ substrates at a low temperature $\sim 550^{\circ} \mathrm{C}$ by adding the catalyst sodium nitrate $\left(\mathrm{NaNO}_{3}\right)$, as sodium precursor. By using this technique, we can grow single and polycrystals of $\mathrm{MoS}_{2}$ controlling the synthesis parameters: precursor's ratio and growth time.

We have used the atmospheric pressure chemical vapor deposition system to synthesize the monolayer $\mathrm{MoS}_{2}$ single crystals and polycrystals. We obtained the crystals by the sulfurization of molybdenum oxide. We used the thermal decomposition of sodium nitrate as a source of sodium [175]. The growth process is carried out inside a quartz tube in a dual-zone tubular furnace (figure 3.1). First, we cleaned the substrate following the same procedure as explained in chapter 3, section 3.1.1. Then a small amount (6 mg) of $\mathrm{MoO}_{3}$ (Sigma-Aldrich, $99.97 \%$ pure) powder along with $2 \mathrm{mg}$ sodium nitrate (SigmaAldrich, 99.0\% pure) was put in a mortar pestle and ground for $15 \mathrm{~min}$. We loaded the mixed powder in an alumina crucible. The substrate was placed right on top of the powder mixture. The substrate's position is significant in the growth process. If the substrate is placed high from the powder as usually followed in high-temperature synthesis, the growth will not happen. Still, if the substrate is touching the dust, it will eventually result in the growth of bulk instead of a monolayer. We should place the substrate inside the crucible facing downward, but make sure it is a millimetre high from the powder. Then $300-350 \mathrm{mg}$ of sulfur fine powder (99.5\% pure) is loaded in another aluminium crucible and then both placed inside the quartz tube. The distance between the two cauldrons is $\sim 25 \mathrm{~cm}$. We use the Argon flow rate at $100 \mathrm{sccm}$ inside the quartz tube. During synthesis, the reaction chamber was first heated to $200^{\circ} \mathrm{C}$ in an $\mathrm{Ar}$ atmosphere and left there for $20 \mathrm{~min}$ to remove oxygen and other possible sources of contamination from the air. Then, the furnace was 
Chapter 4. CVD Grown $\mathrm{MoS}_{2}, \mathrm{WS}_{2}$ Monolayer, and their Vertical Heterostructure

heated independently with the rate of $33^{\circ} \mathrm{C} / \mathrm{min}$ until it reaches $\sim 550^{\circ} \mathrm{C}$ for $\mathrm{MoO}_{3}+\mathrm{NaNO}_{3}$ powder and $250^{\circ} \mathrm{C}$ for sulfur powder. Keeping the materials under the respective constant temperatures for the next $12 \mathrm{~min}$, then let it cool down naturally. The flow rate of Ar was kept constant at $100 \mathrm{sccm}$ during the whole growth process.

(a) $\mathrm{NaNO}_{3} / \mathrm{MoO}_{3}$
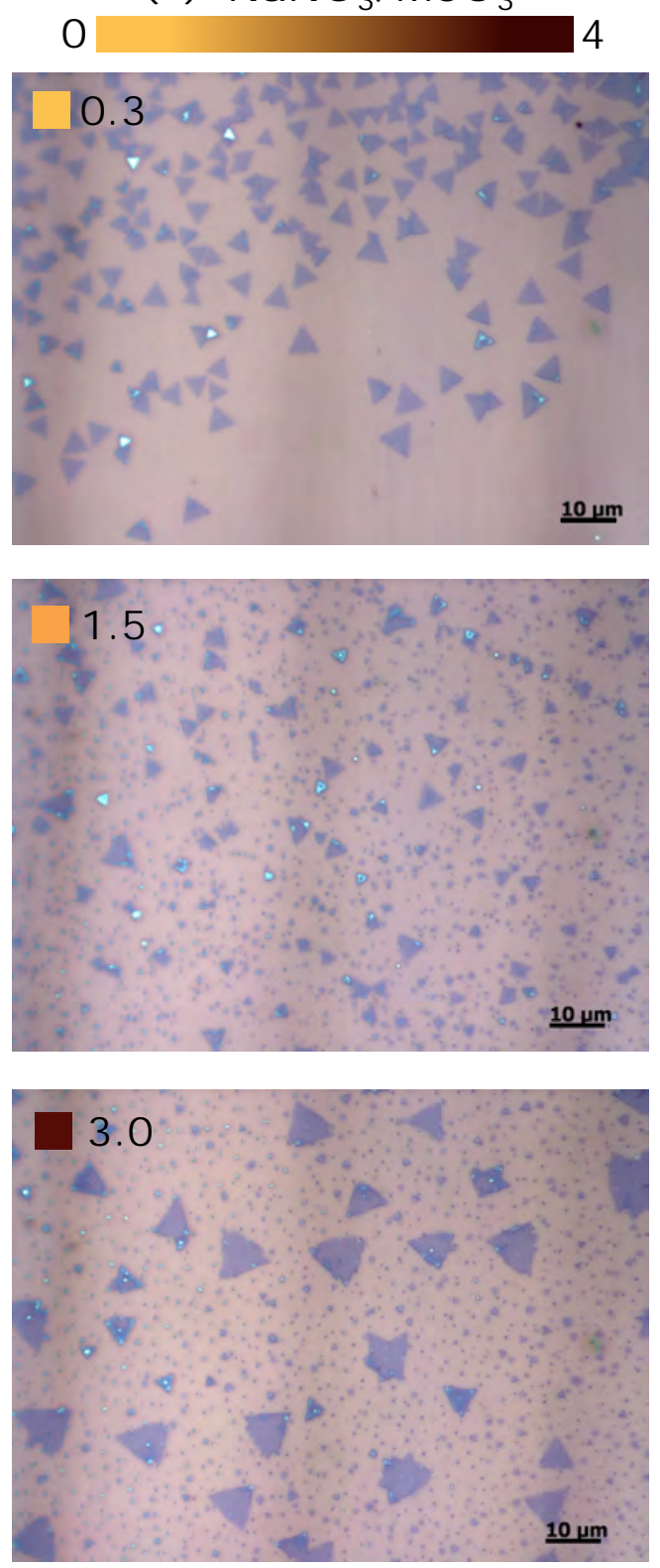

(b) Time (min)
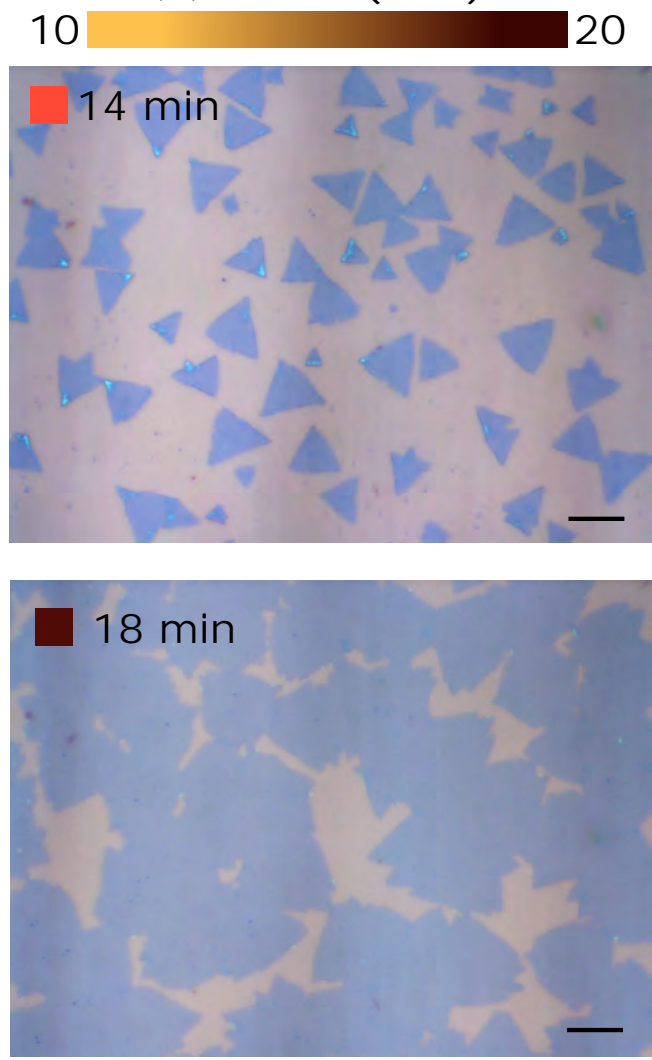

$20 \min$

Figure 4.7: Dependence of synthesis parameters in the growth process. (a) Optical images of single-crystalline $\mathrm{MoS}_{2}$ changing the precursor's ratio $\mathrm{NaNO}_{3} / \mathrm{MoO}_{3}$ from 0.3 to 3.0. (b) Optical images show the island coalescence and form the polycrystal $\mathrm{MoS}_{2}$ by increasing the time from 14 to $20 \mathrm{~min}$, scale bar $10 \mu \mathrm{m}$.

Finally, $\mathrm{MoS}_{2}$ single and polycrystals were grown on $\mathrm{SiO}_{2} / \mathrm{Si}$ substrates 
at $\sim 550^{\circ} \mathrm{C}$. The synthesis parameters: $\mathrm{NaNO}_{3} / \mathrm{MoO}_{3}$ precursor's ratio and growth time play an important role in the synthesis process. Figure 4.7(a) shows three optical images of $\mathrm{MoS}_{2}$ grown using different ratios between $\mathrm{NaNO}_{3}$ and $\mathrm{MoO}_{3}$ precursors at a fixed 12 min growth time. We observed that for ratios $>1$, a large number of nucleation sites (small dots in the second and third images) did not have an ideal grown process, and some of the triangles have multiple layers at the corners. However, for ratio 0.3, we can find monolayers in perfect triangular shape. The average size of a triangle is approximately $5 \mu \mathrm{m}$. Two scanning electron micrograph images, see figure 4.8, confirm the morphology of $\mathrm{MoS}_{2}$ samples. Optical images in figure 4.7(b) shows the $\mathrm{MoS}_{2}$ grown at different growth times by keeping the ratio equal to 0.3. By increasing the growth time from $14 \mathrm{~min}$ to $20 \mathrm{~min}$, the size of the triangles keeps on growing and eventually merge to form a uniform polycrystalline $\mathrm{MoS}_{2}$ film. From these experiments, we have found that the ideal growth parameters are precursor ratio 0.3 with growth time 12 min to single crystals and $20 \mathrm{~min}$ to polycrystalline films at temperature $\sim 550^{\circ} \mathrm{C}$.
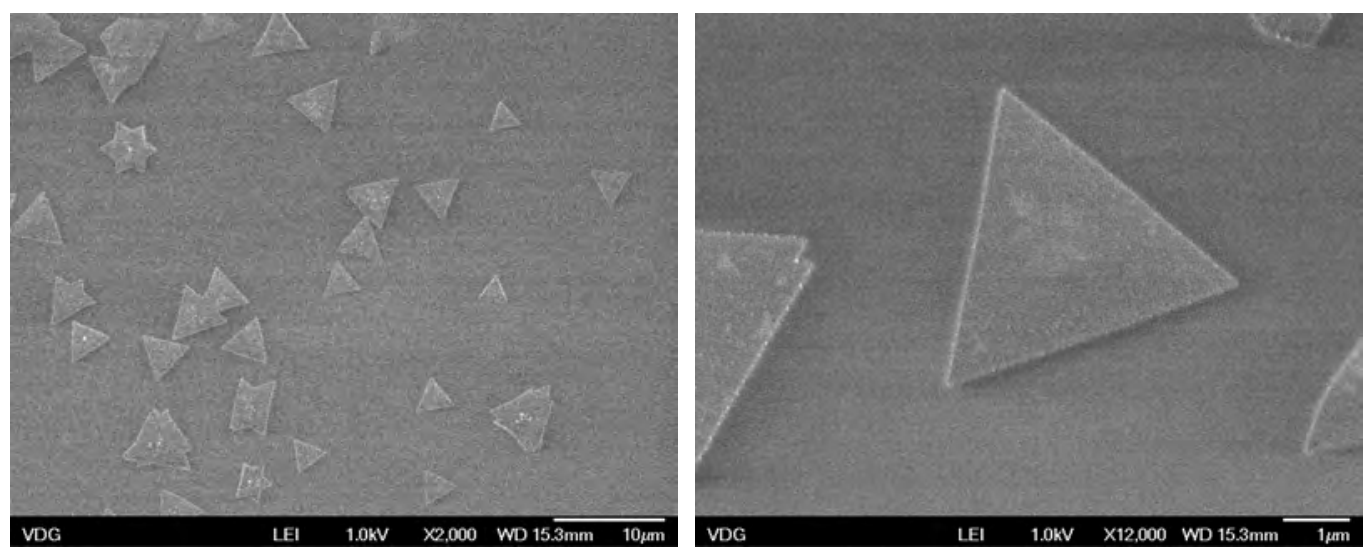

Figure 4.8: SEM images of $\mathrm{MoS}_{2}$ single crystals.

We acquired X-ray photoelectron spectroscopy (XPS) spectra to examine the presence of sodium after the grown process. Figure 4.9(a) shows the XPS spectra of $\mathrm{MoS}_{2}$ film. The detailed XPS spectra and fits for Mo and S peaks are shown in figure $4.9(\mathrm{c}, \mathrm{d})$. The characteristic peaks at 229.4 and $232.5 \mathrm{eV}$ corresponds to molybdenum $3 \mathrm{~d}^{5 / 2}$ and $3 \mathrm{~d}^{3 / 2}$ and peaks at $162.6 \mathrm{eV}$ and 163.8 $\mathrm{eV}$ are in line with sulfur $2 \mathrm{p}^{3 / 2}$ and $2 \mathrm{p}^{1 / 2}$, respectively. The peaks positions agree with the previous report [176]. We found a small amount of sodium by observing the peak at $1073 \mathrm{eV}$. To clarify the origin of sodium, we did a water bath to our crystals for $2 \mathrm{hr}$ and retook the XPS. After the bath, we do not observed the sodium on the XPS spectrum, see figure 4.9(b). This evidence 
Chapter 4. CVD Grown $\mathrm{MoS}_{2}, \mathrm{WS}_{2}$ Monolayer, and their Vertical Heterostructure

clarifies that sodium is only acting as a mediator in the growth process and can not found on the crystalline lattice of $\mathrm{MoS}_{2}$. Besides that, the synthesis does not occur at $\sim 550^{\circ} \mathrm{C}$ without sodium. Hence, the sodium promotes a catalytic chemical reaction and the reduction of synthesis temperature.
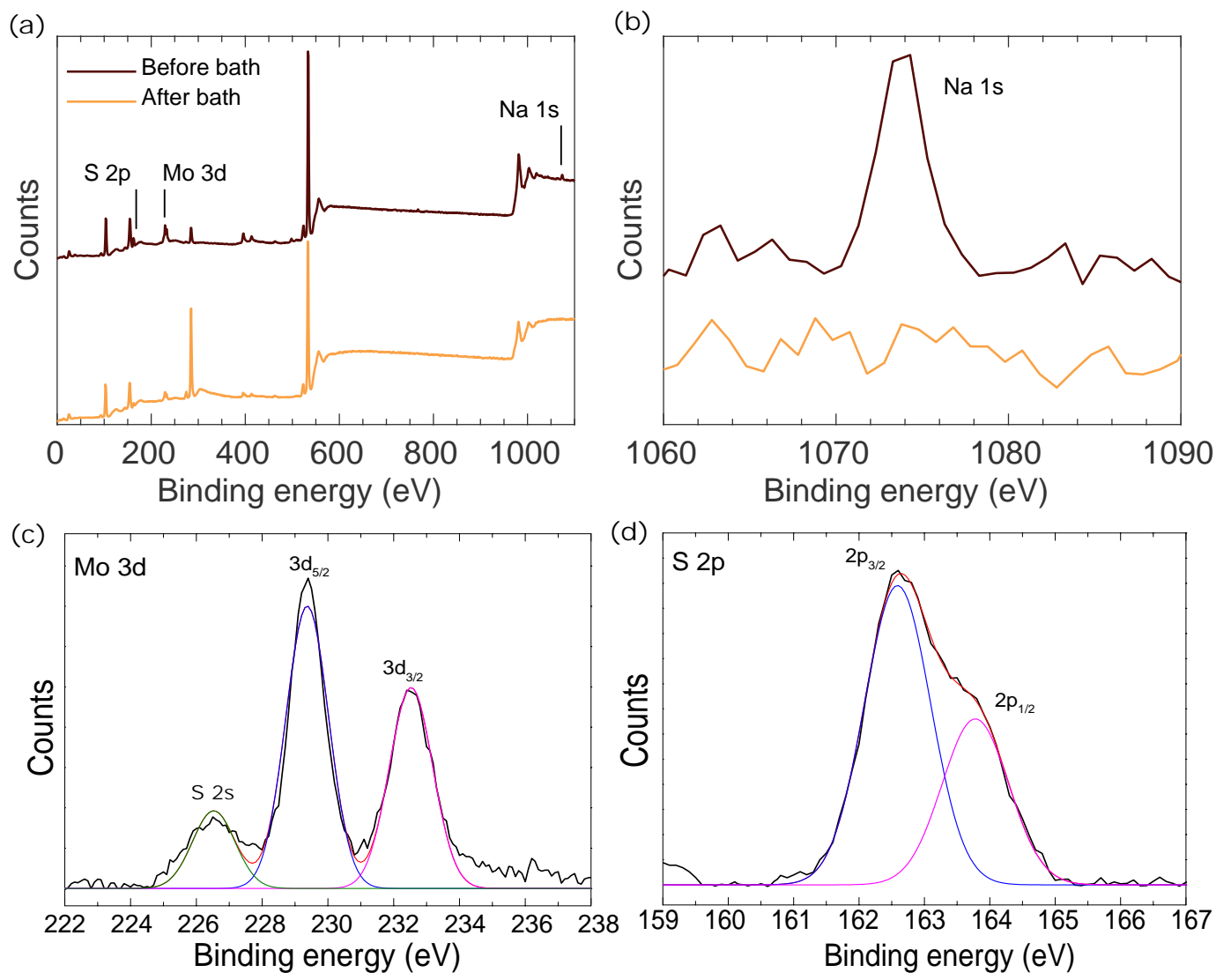

Figure 4.9: XPS experiments. (a) XPS spectra of the grown sample before and after water bath for $2 \mathrm{hr}$. (b) Detailed XPS spectra of Na 1s peak before and after water bath. Detailed XPS spectra and fitting of Mo and S peaks. (c) Molybdenum 3d spectrumof $\mathrm{MoS}_{2}$ grown on $\mathrm{SiO}_{2} / \mathrm{Si}$ substrate. (d) S 2p spectrum of the same sample. 


\subsection{1}

\section{Catalytic Behaviour of Na Explained by DFT Calculations}

In order to understand the role of the $\mathrm{Na}$ atoms on the growth of the $\mathrm{MoS}_{2}$ structures, we perform a DFT calculation of the formation energy, as function of the sodium concentration. A $3 \times 3 \mathrm{MoS}_{2}$ supercell crystal structure is built, and the $\mathrm{Na}$ atoms are initially placed parallel to the Mo plane, in the direction of the center of hexagons. We relax the whole structure minimizing the energy and the forces keeping the supercell size fixed and, after that, we compute the total energy of the final structures of sodium atoms and the $\mathrm{MoS}_{2}$ supercell constrained by the $\mathrm{Na}$ in a separately way. The formation energy is given by $E_{\text {form }}=\left[E\left(\mathrm{MoS}_{2}+\mathrm{Na}\right)\right]-\left[E\left(\mathrm{MoS}_{2}\right)+E(\mathrm{Na})\right]$, and for all concentrations of $\mathrm{Na} / \mathrm{MoS}_{2}$ we find that $E_{\text {form }}<0$, which confirms that the presence of the $\mathrm{Na}$ is favorable for the formation of $\mathrm{MoS}_{2}$.

In our model, the $\mathrm{Na}$ atoms form a hexagonal lattice that provides nucleation sites to the formation of $\mathrm{MoS}_{2}$. The theoretical lattice parameter of the $\mathrm{MoS}_{2}$ is $a_{\mathrm{MoS}_{2}}=3.237 \AA$, while for the hexagonal lattice of Na atoms the optimal distance that minimizes the energy is $a_{\mathrm{Na}}=3.655 \AA$. This value is close to the separation between $\mathrm{Na}$ atoms in the (111) plane of the Face Centered Cubic (FCC) crystal cell of $\mathrm{NaCl}(\approx 4 \AA)$ and is also close, but a slight large, then the $\mathrm{MoS}_{2}$ lattice parameter. In this way, a large concentration of $\mathrm{Na}$ provides more nucleation sites, but the $\mathrm{S}$ atoms close to the $\mathrm{Na}$ will be more constrained. On the other hand, large supercells of the $\mathrm{MoS}_{2}$ are very unlikely to be produced in the low concentration regime because the Na atoms tend to be close to each other.

Figures 4.10(a-f) show structures after the energy minimization. For the 0.1 ratio in figure 4.10(a) the single $\mathrm{Na}$ atom is exactly in the direction of the center of one of the hexagons, and it is $3 a_{\mathrm{MoS}_{2}}$ away to the next neighbor (not shown in the figure). In the real synthesis procedure, $\mathrm{MoO}_{3}$ and $\mathrm{NaNO}_{3}$ ratio can be controlled, but not the position of $\mathrm{Na}$ sites after the reaction, which means that sparse distributions like in figure 4.7(a) in large systems is very unlikely (with minimal entropy), and only small supercells can be grown in this concentration. In figure 4.10(b) the two Na atoms are close one to each other. However, it is not exactly in the direction of the center of the hexagons. The relative position change from $3.237 \AA$ to $3.543 \AA$ in the energy minimization process, while the changes in the position of the Mo and $\mathrm{S}$ atoms are around $0.1 \%$. The change in the relative position between atoms in the $\mathrm{MoS}_{2}$ is also small for the 0.3 ratios shown in figure $4.10(\mathrm{c})$ and the average distance between $\mathrm{Na}$ atoms is $3.578 \AA$, while between the closest Mo and S atoms are $3.216 \AA$ and $3.245 \AA$, respectively. For the other concentrations in figures 4.10(a-f), the 
change in the Mo and S position are considerably large, and this effect can be seen in figure $4.10(\mathrm{~g})$.

(a)

(d) (b)

(e) (c)

(f)

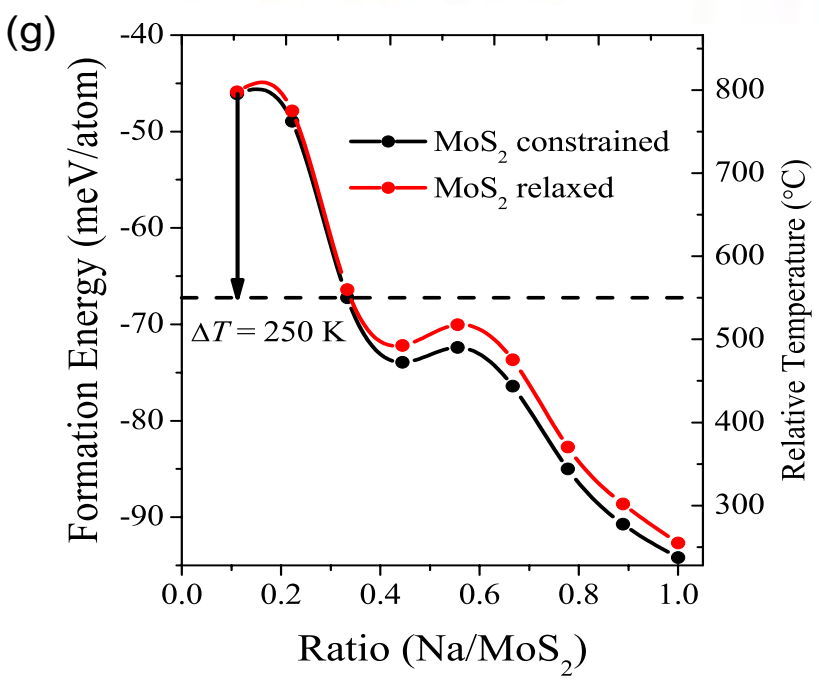

Figure 4.10: DFT calculations of the formation energy. (a-f) Atomic positions after minimization of the energy and forces in a $3 \times 3$ fixed size supercell of $\mathrm{MoS}_{2}$ in the presence of $\mathrm{Na}$ atoms. The light blue, dark grey and dark yellow balls represent the $\mathrm{Na}, \mathrm{Mo}$, and $\mathrm{S}$ atoms, respectively. (g) Formation energy per atom computed with the $\mathrm{MoS}_{2}$ constrained by the presence of the Na after the minimization (black circles) and fully relaxed (red circles). The full black and red lines are guides to the eyes. The vertical axis on the right side is a conversion of energy to temperature.

We plot the formation energy per atom for different ratios of $\mathrm{Na}$ and $\mathrm{MoS}_{2}$ in figure $4.10(\mathrm{~g})$. In this Figure, the black circle is the formation energy computed when the presence of the Na still constrains the $\mathrm{MoS}_{2}$ system, and the red circles are computed using the fully relaxed $\mathrm{MoS}_{2}$ lattice. We see that the difference in energy between the relaxed and constrained systems increases 
(a)

(d) (b)

(e) (c)

(f)

Figure 4.11: Final structures for large concentrations of $\mathrm{Na}$ in $\mathrm{MoS}_{2}$. (a-c) top view of the $3 \times 3 \mathrm{MoS}_{2}$ supercell with 7, 8, and $9 \mathrm{Na}$ atoms, respectively. (d-f) side view of the same structures in (a-c), respectively. The average distance between $\mathrm{Na}$ atoms and the plane with Mo are approximately $4.0 \AA$ (first layer) and $5.8 \AA$ (second layer). For the low concentration regimes (i.e. $<0.45$ ) the average distance varies approximately linearly between $3.7 \AA$ and $4.0 \AA$ with the number of the $\mathrm{Na}$ atoms in the supercell.

with the $\mathrm{Na}$ concentration indicating that the lattice is more distorted in this regime. Figure $4.10(\mathrm{~g})$ also shows that the formation energy decreases drastically, as expected when more Na sites are present. At the ratio 0.3 , the $\mathrm{MoS}_{2}$ lattice is almost unchanged, with a decrease in the formation energy of the same order of the decrease in temperature $\left(\Delta T=250^{\circ} \mathrm{C}\right)$ which fully agree with the experimental ideal growth parameters discussed before. For concentrations larger than 0.7 , our calculations indicate that some $\mathrm{Na}$ are not on the same plane as the others, and due to the repulsion between a large number of atoms in a small confinement space (see figure 4.11), the minimal energy configuration starts to have two layers of sodium. Although these configurations present smaller formation energies, the $\mathrm{Na}$ atoms located in the extra layer does not play a fundamental role in the formation of large $\mathrm{MoS}_{2}$ cells because they are far away from the molybdenum and sulfur atoms.

(The DFT calculations for our system was done by Prof. Marcus V. O. Moutinho from UFRJ. DFT calculations were performed in the generalized gradient approximation of the exchange-correlation effect. The QUANTUMESPRESSO package [177] was used with ultrasoft Perdew-Burke-Ernzerhof pseudopotentials and plane-wave cutoff energy of $60 \mathrm{Ry})$. 
Chapter 4. CVD Grown $\mathrm{MoS}_{2}, \mathrm{WS}_{2}$ Monolayer, and their Vertical

\subsection{2}

\section{Chemical Equations Explaining the Growth}

Within the subject process (1), some parallel reactions occur, as followings:

$$
2 \mathrm{MoO}_{3}+7 \mathrm{~S} \longrightarrow 2 \mathrm{MoS}_{2}+3 \mathrm{SO}_{2} \uparrow \quad---(1)
$$

At the temperatures higher than $380^{\circ} \mathrm{C}$, in parallel with the main reaction (1), sodium nitrate undergoes decomposition (2) and free oxygen forms nitrogen(IV) oxide (gas).

$$
2 \mathrm{NaNO}_{3} \stackrel{380^{\circ} \mathrm{C}}{\longrightarrow} 2 \mathrm{NaNO}_{2}+\mathrm{O}_{2} \uparrow \quad---(2)
$$

In parallel, solid $\mathrm{MoO}_{3}$ reacts with sodium nitrite, whereas water-soluble sodium molybdate is formed. The gaseous products $\left(\mathrm{NO}_{2}\right.$ and $\left.\mathrm{SO}_{2}\right)$ are removed with Ar flow.

$$
4 \mathrm{NaNO}_{2}+\mathrm{O}_{2}+2 \mathrm{MoO}_{3} \longrightarrow 2 \mathrm{Na}_{2} \mathrm{MoO}_{4}+4 \mathrm{NO}_{2} \uparrow \quad--(3)
$$

This $\mathrm{Na}_{2} \mathrm{MoO}_{4}$ is now present as contamination on the substrate which is evident in XPS, $\mathrm{Na}_{2} \mathrm{MoO}_{4}$ is soluble in water by giving water bath to the sample we can remove $\mathrm{Na}^{+}$.

\subsection{3}

\section{Characterization of Na Mediated Synthesized Samples}

We turn our attention to investigate the structural and optical properties of $\mathrm{MoS}_{2}$ single crystals. Figure 4.12(a) shows the AFM image of a triangle, the line profile inset is uniform and has a height of $1.0 \mathrm{~nm}$, which confirms the monolayer thickness $[11,15,28]$. Figure 4.12(b) shows one representative Raman spectrum $(473 \mathrm{~nm})$ obtained from the same $\mathrm{MoS}_{2}$ monolayer. The recorded Raman spectrum exhibited the in-plane phonon mode $\mathrm{E}^{\prime}$ at $379.7 \mathrm{~cm}^{-1}$ and the out-of-plane $\mathrm{A}_{1}^{\prime}$ mode at $399.7 \mathrm{~cm}^{-1}$ [96]. The difference in peak position between these modes is $20 \mathrm{~cm}^{-1}$, which corresponds to monolayer [166]. The FWHM of $E^{\prime}$ peak has a value of $3.4 \mathrm{~cm}^{-1}$, indicating high crystalline quality with no amorphous phase [99]. We performed a Raman mapping over the same triangle. Figures $4.12(\mathrm{c})$ and (d) present the peak intensity map of $\mathrm{A}_{1}^{\prime}$ and $\mathrm{E}^{\prime}$ 
Chapter 4. CVD Grown $\mathrm{MoS}_{2}, \mathrm{WS}_{2}$ Monolayer, and their Vertical Heterostructure

modes, respectively. The maps show a uniform intensity distribution inside the triangle, while at the corners, the intensity is lower, most likely due to the edge defects $[178,179]$. The peak position maps of these modes are shown in figure 4.12(e,f), showing uniformity throughout the area of the triangles. These maps are evidence of the uniform structural quality of the crystal.

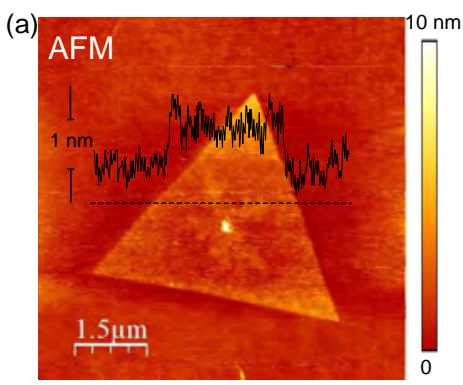

(d)
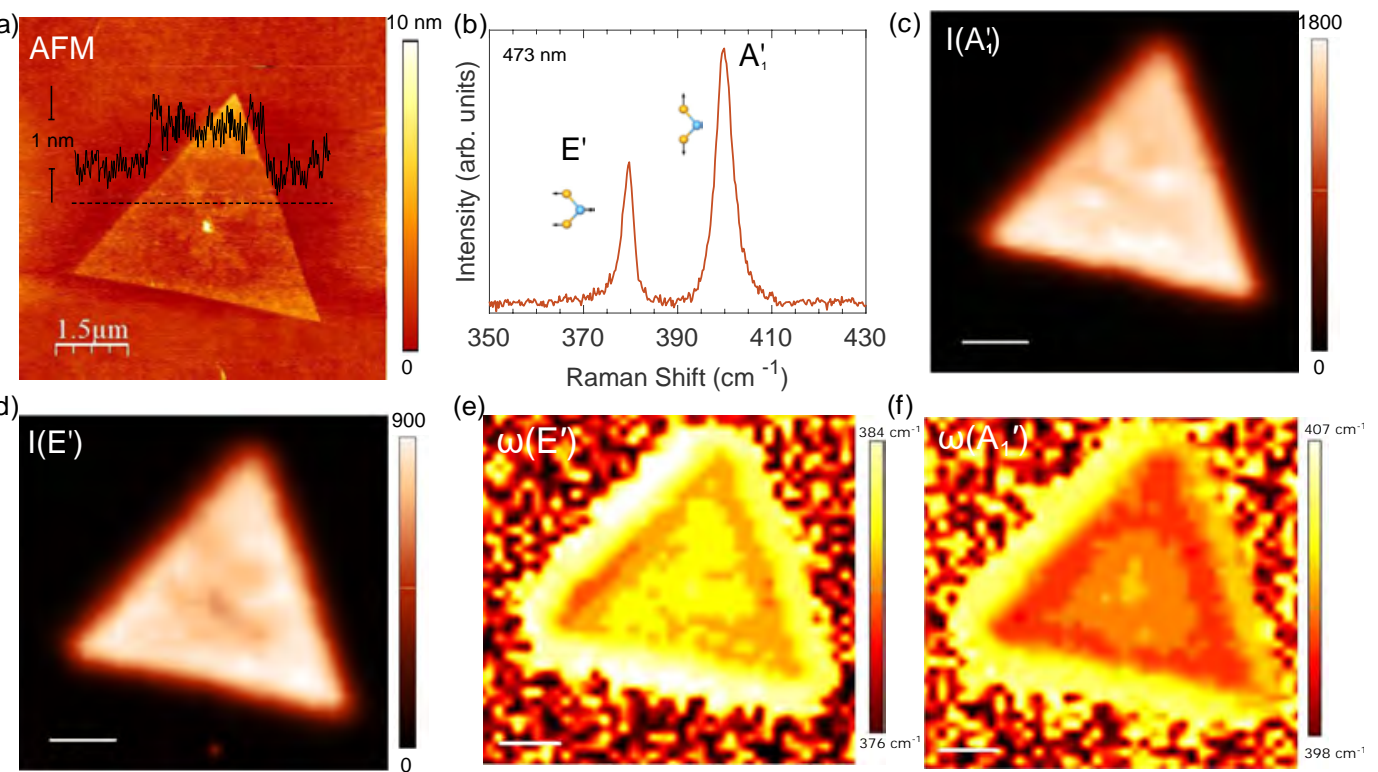

(g)

(h)
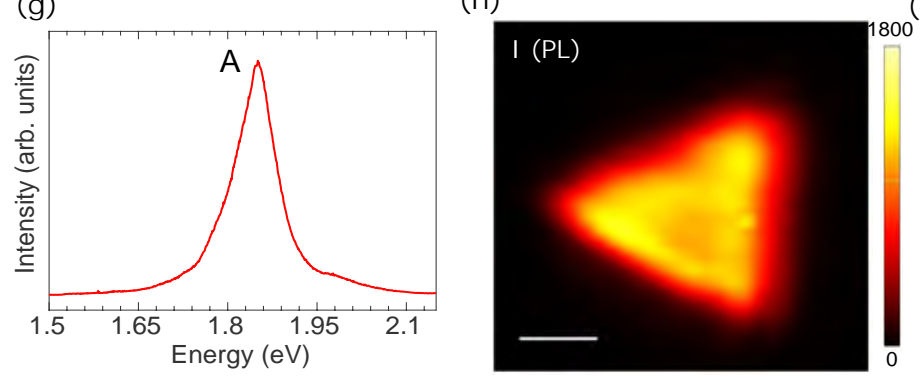

(i)

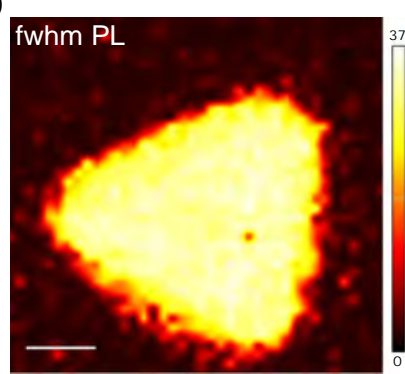

Figure 4.12: Characterization of single crystal. (a) AFM image of a monolayer of $\mathrm{MoS}_{2}$, inset the line profile shows the step of $1 \mathrm{~nm}$. (b) Raman spectra of $\mathrm{MoS}_{2}$ monolayer from $473 \mathrm{~nm}$ laser line took from (a). (c) mapping of $\mathrm{A}_{1}^{\prime}$ intensity mode and (d) $\mathrm{E}^{\prime}$ intensity mode, scale bar $1 \mu \mathrm{m}$. (e) The peak position maps of $\mathrm{E}^{\prime}$ and (f) $\mathrm{A}_{1}^{\prime}$ modes showing uniformity throughout the triangles area.(g) Photoluminescence spectra took of $\mathrm{MoS}_{2}$. (h) PL intensity map of A exciton, scale bar $1 \mu \mathrm{m}$. (i) fwhm of Photoluminescence.

Figure 4.12(g) shows the photoluminescence spectra of the $\mathrm{MoS}_{2}$ triangle exhibiting a A exciton peak at $670 \mathrm{~nm}(1.85 \mathrm{eV})$. The intense photoluminescence is a result of the direct bandgap present in monolayer [11,102,173]. A single, sharp, and intense peak in the PL spectrum indicates our sample's high optical quality. Furthermore, the PL mapping of the corresponding triangle also indicates uniformity at PL emission see figure 4.12(i). The uniform in- 
Chapter 4. CVD Grown $\mathrm{MoS}_{2}, \mathrm{WS}_{2}$ Monolayer, and their Vertical Heterostructure

tensity overall triangle is an indicator of the identical thickness. All the maps demonstrate that this entire $\mathrm{MoS}_{2}$ crystal is an equal monolayer triangle.

Further, we investigated the polycrystalline monolayer $\mathrm{MoS}_{2}$ formed by the coalescence of triangles shown in figure 4.7(b). The Raman and Photoluminescence spectra of the film, indicates the crystalline structure and confirm the monolayer thickness. We have selected different areas on the $\mathrm{MoS}_{2}$ film and did AFM, Raman, and PL mappings. The AFM shows a uniformly flat surface over the crystal, see figure 4.13(a). The small holes appearing on the image could result from the not perfect matching between triangles during the coalescence.

(a)

(c)
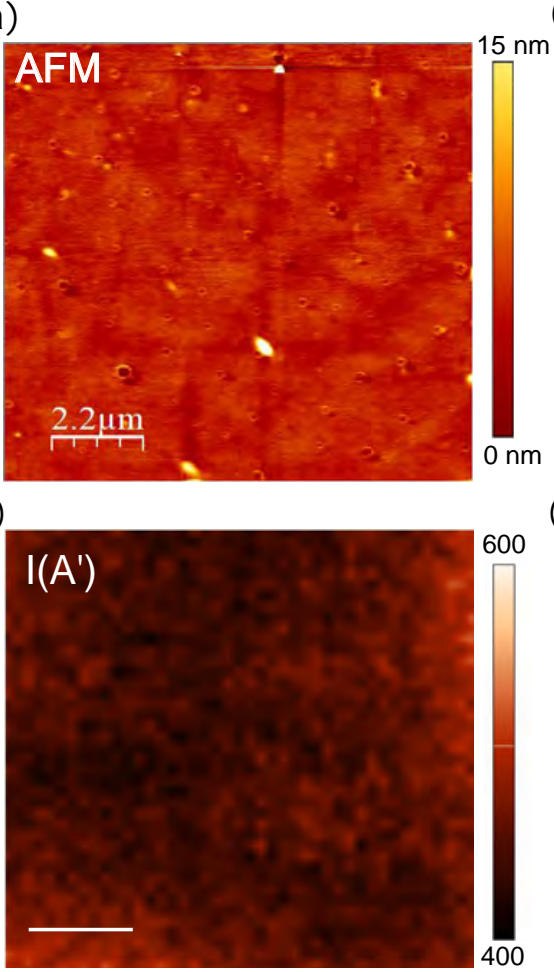

(e)

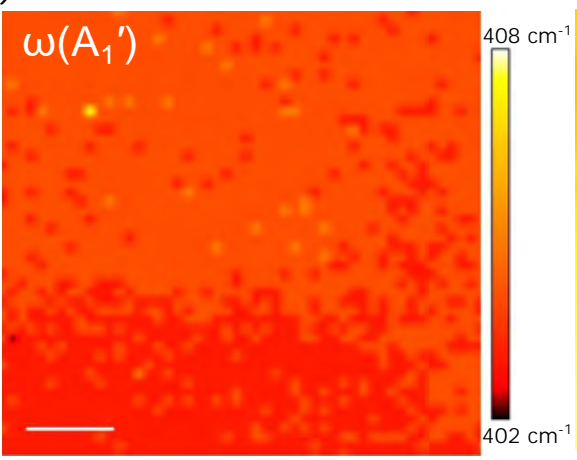

(b)

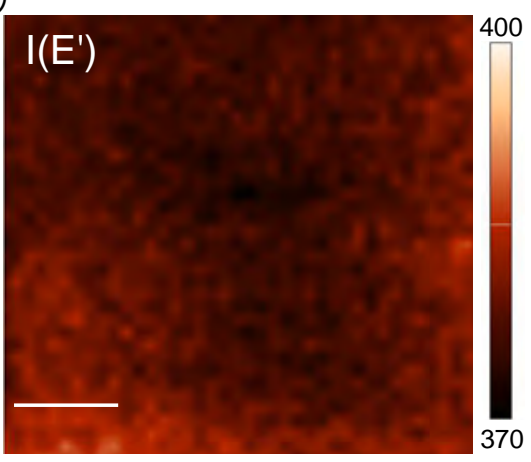

(d)

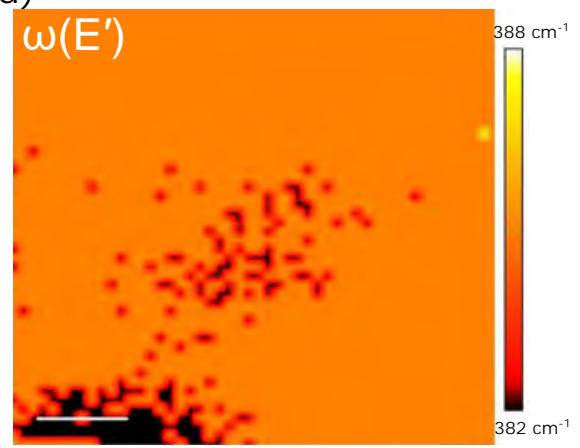

(f)

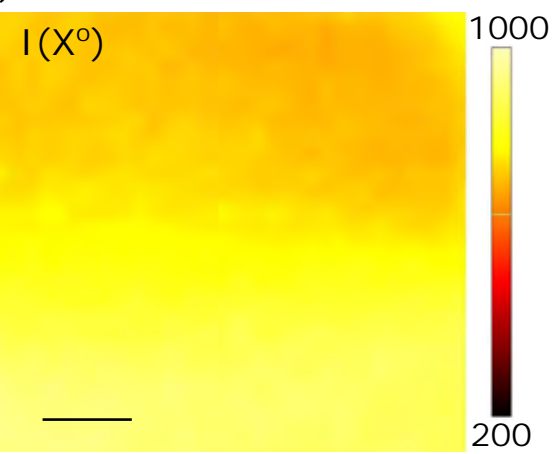

Figure 4.13: Characterization of polycrystal. (a) AFM image of polycrystalline $\mathrm{MoS}_{2}$. (b) Raman mapping of $\mathrm{E}^{\prime}$ intensity mode and (c) $\mathrm{A}_{1}^{\prime}$ intensity mode, scale bar $2 \mu \mathrm{m}$. (d) peak position maps of $\mathrm{E}^{\prime}$ and, (e) $\mathrm{A}_{1}^{\prime}$ mode (f) PL intensity map, scale bar $2 \mu \mathrm{m}$. 
Moreover, Raman mapping on the intensity of $\mathrm{E}^{\prime}$ and $\mathrm{A}_{1}^{\prime}$ modes and peak position maps of the corresponding sample $\mathrm{E}^{\prime}$ and $\mathrm{A}_{1}^{\prime}$ modes shows uniform color contrast see figures 4.13(b-e), which demonstrates its perfect thickness uniformity and crystallinity over a large scale. The PL intensity map is shown in figure 4.13(f). The results show that increasing the growth time, it will possibly achieve a uniform monolayer $\mathrm{MoS}_{2}$ film.
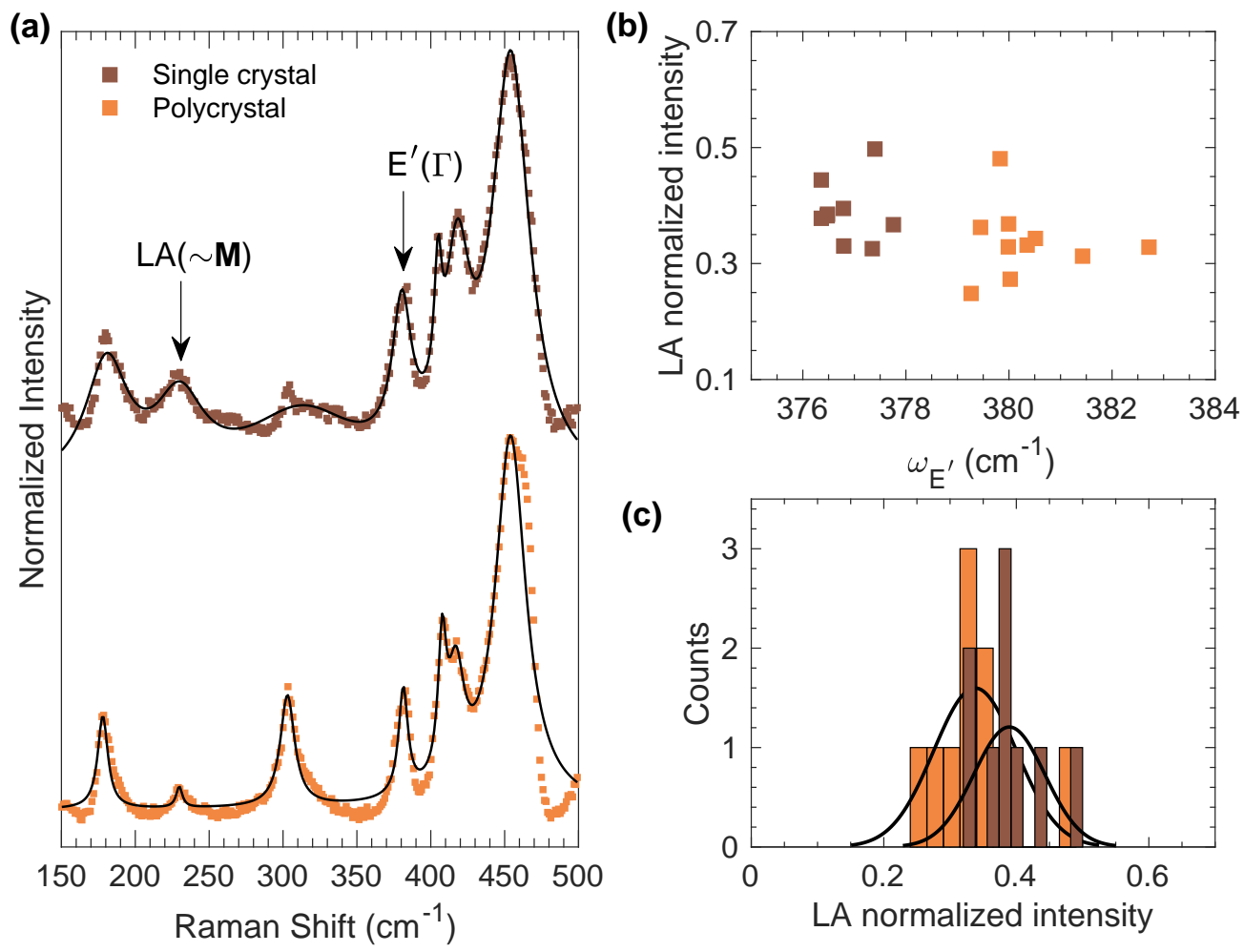

Figure 4.14: Analysis of defects using Raman spectroscopy. (a) Raman spectra of single and polycrystal of $\mathrm{MoS}_{2}$ with the fitting. (b) Normalized intensity of LA mode in the function of $\mathrm{E}^{\prime}$ frequency. (c) Histograms of LA mode of single and polycrystals.

We acquired Raman spectra using the $638 \mathrm{~nm}$ laser line to study the defects on our as-grown single and polycrystals. Figure 4.14(a) presents the representative Raman spectra of single crystal and polycrystal. The mode at $\sim 379 \mathrm{~cm}^{-1}$ corresponds to $\mathrm{E}^{\prime}$. The peak at $228 \mathrm{~cm}^{-1}$ is defect related LA mode near $\mathbf{M}$ point at Brillouin zone. We took Raman spectra of ten different single crystal and ten from areas at a polycrystalline film to quantify the structural defects. We use a Lorentzian function to adjust the Raman spectra, and the disorder-activated LA(M) mode was normalized with the corresponding first-order $\mathrm{E}^{\prime}$ mode. In figure 4.14(b), we plotted the normalized intensity of LA peak, as a function of $\mathrm{E}^{\prime}$ frequency. We observe two distinct sets of data. The $\sim 3 \mathrm{~cm}^{-1}$ blueshift of $\mathrm{E}^{\prime}$ mode of polycrystal when compare 
to single crystals indicate the presence of strain [180]. Probably, the strain comes from the coalescence of triangles during the grown process, showing in figure 4.14(b). The histograms in figure 4.14(c) shows the distribution of LA normalized intensity for both sets of samples. We found the average intensity ratio for single crystal 0.39 while for polycrystal 0.34 . Hence, the LA mode intensity points out a similar number of defects between single crystals and polycrystals.

We also investigated the electrical properties of our crystals using the two probe devices fabricated on the as-grown sample, the detailed results are given in chapter 6. Our devices show high electrical performance and the mobility is estimated as $9.8 \pm 0.7 \mathrm{~cm}^{2} V^{-1} \mathrm{~s}^{-1}$ for single crystal and $2.7 \pm 0.5 \mathrm{~cm}^{2} V^{-1} \mathrm{~s}^{-1}$ for polycrystal which is comparable with the previous reports $[28,32,69,181]$. The relatively low electron mobility of the film is likely due to the presence of domain boundaries in the polycrystals, which could trap electrons and affect the charge transfer. Typically, in high-temperature synthesis, it is common to observe gate voltage leakage through pinholes caused by the destruction of a thin $\mathrm{SiO}_{2}$ layer. Another advantage of low-temperature synthesis is the possibility to direct fabrication of the device, avoiding the transfer procedure.

\section{5}

\section{Synthesis of $\mathrm{MoS}_{2}$ on Glass}

We have discussed so far, sodium assisted growth which enables us to decrease the synthesis temperature. This encouraged us to move on to the direct and low temperature synthesis on the glass. The transition temperature of ordinary glass is $564^{\circ} \mathrm{C}$, so a recipe that works below this temperature is needed. We synthesized $\mathrm{MoS}_{2}$ triangular monolayer on glass at low temperature $\sim 520^{\circ} \mathrm{C}$, by direct sulfurization of $\mathrm{MoO}_{3}$ powder. By keeping in mind the catalytic behaviour of $\mathrm{Na}$ we have chosen borosilicate glass (composed of, 80 $\%$ silica, $13 \%$ boric oxide, $4 \%$ sodium oxide, and $3 \%$ aluminium oxide) as a substrate. The synthesis process is the same for glass as explained in $\mathrm{Na}$ mediated synthesis process except adding $\mathrm{NaNO}_{3}$, because glass itself has $\mathrm{Na}$ which can act as nucleation sites and helps in low temperature synthesis.

First of all the grown sample was analyzed by optical microscopy. An optical image of $\mathrm{MoS}_{2}$ glass sample is shown in figure 4.15(a) showing most of the triangles have multiple layers with very few monolayer triangles. As explained earlier by DFT calculations large amount of sodium although helps in decreasing the synthesis temperature, but starts growing multiple layers instead of single layer triangular islands. The higher density of multiple layers present in our sample is a result of higher sodium concentration in borosilicate 
(a)

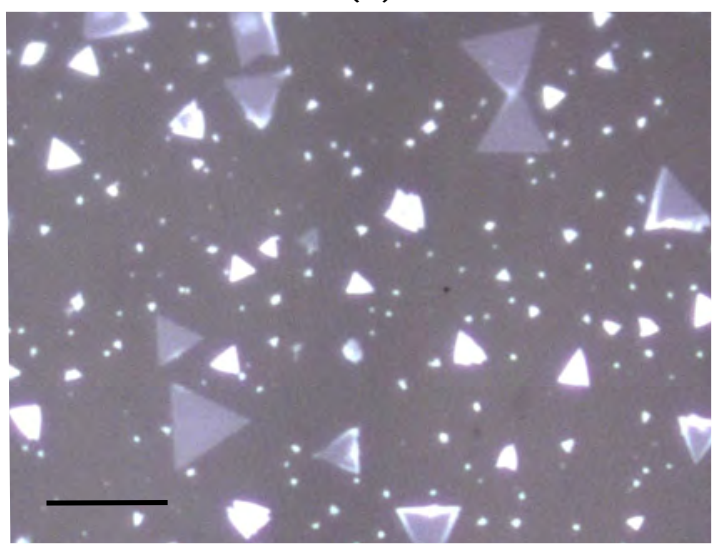

(c)

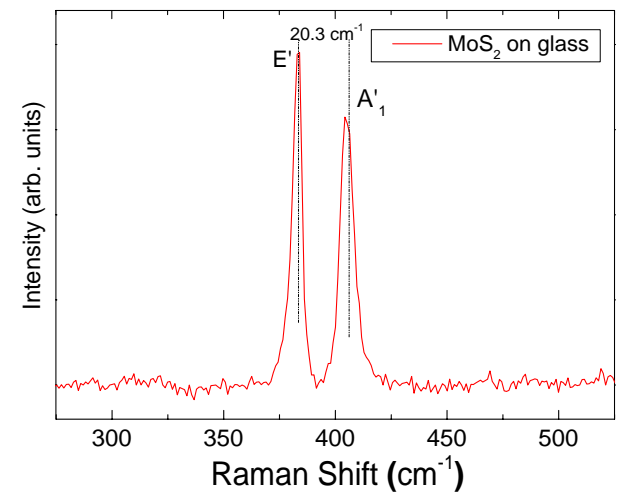

(e) (f) (b)

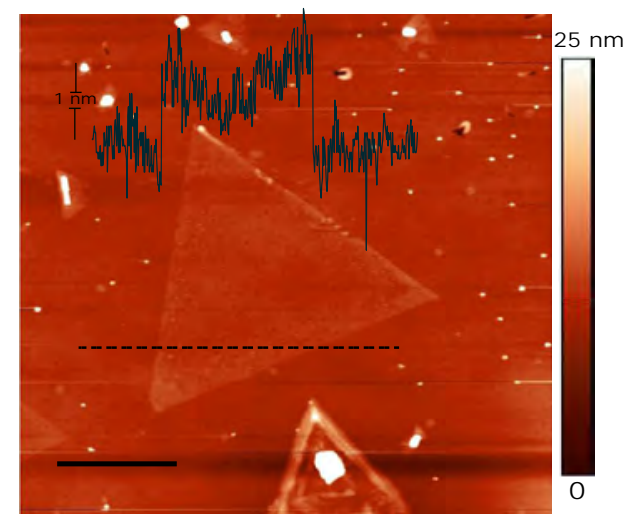

(d)

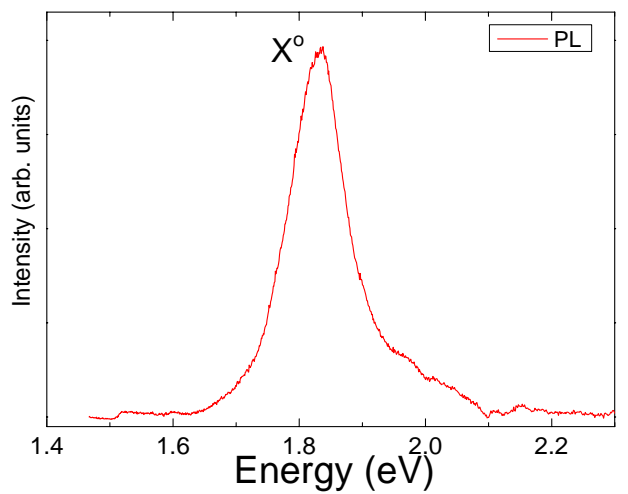

(g)
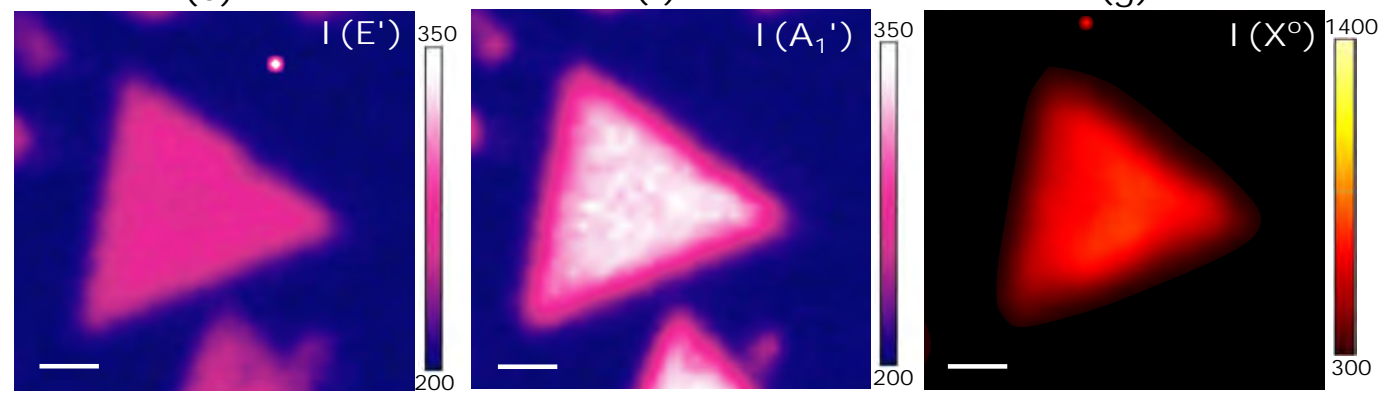

Figure 4.15: (a) Optical image $\mathrm{MoS}_{2}$ on glass scale bar is $10 \mu \mathrm{m}$. (b) AFM image of a monolayer triangle with height of $\sim 1 \mathrm{~nm}$ scalr bar is $2 \mu \mathrm{m}$. (c) Raman and, (d) PL spectra of the corresponding triangle. (e) Intensity map of $E^{\prime}$, (f) $A_{1}^{\prime}$ modes of the same triangle. (g) PL intensity map.

glass. Monolayer triangles can be easily identified because of larger in size and lighter color contrast compared to its counterpart. The triangles were further confirmed as monolayer by AFM, Raman, and PL spectroscopy. The AFM image of the corresponding triangle is shown in figure 4.15(b) showing a uniform surface with average height is estimated to be around $\sim 1 \mathrm{~nm}$ which corresponds to monolayer. A small variation in height of about $3 \AA$ is likely due to the roughness of glass because glass is not as smooth as $\mathrm{SiO}_{2}$ substrate. Representative Raman spectrum obtained from the same monolayer $\mathrm{MoS}_{2}$ 
triangle is shown in figure 4.15(c). The recorded raman spectrum exhibited the characteristic in-plane phonon mode $\mathrm{E}^{\prime}$ and the out-of-plane $\mathrm{A}_{1}^{\prime}$ modes at $386.3 \mathrm{~cm}^{-1}$ and $406.6 \mathrm{~cm}^{-1}$ The difference in peak position between these modes is $20.3 \mathrm{~cm}^{-1}$. The Raman mapping of the same triangle is also recorded by scanning all over the triangle. The peak intensity map of $\mathrm{E}^{\prime}$ and $\mathrm{A}_{1}^{\prime}$ modes is shown in figure $4.15(\mathrm{e}, \mathrm{f})$. The $\mathrm{E}^{\prime}$ mode has uniform intensity throughout the triangle. The $\mathrm{A}_{1}^{\prime}$ mode shows a brighter color at the center, while at the corners the intensity is lower which is most likely due to the edge effect [179]. The tensile strain in the outer region is stronger than that of the inner region hence effecting the intensity. These maps are evidence of uniform structural quality of the crystal.

Figure 4.15(d) shows the PL spectrum of $\mathrm{MoS}_{2}$ triangle on glass substrate, which exhibits a single sharp peak located at $1.85 \mathrm{eV}$ confirming the direct band. Furthermore, the PL mapping of the corresponding triangle also indicates uniformity at PL emission see figure 4.15(g). The uniform colour intensity overall triangle is an indicator of the uniform thickness. All the maps clearly demonstrate that this entire $\mathrm{MoS}_{2}$ crystallite is a uniform monolayer triangle. More studies are needed on glass synthesis to achieve all monolayer triangles. One way is to use the glass substrate with low Na concentration so that the rate of growing multilayer could be decreased.

\section{6}

\section{Partial Conclusion}

Reliable direct synthesis atmospheric pressure CVD methods were proposed to grow monolayer $\mathrm{MoS}_{2}, \mathrm{WS}_{2}$, and their vertical heterostructure. A number of initial attempts were unsuccessful. The most likely reasons were the wrong positions of the substrate, mixing of the crucible, amount of precursor and temperature, etc. For each TMD separate crucible and quartz tube should be used. Initial attempts at $\mathrm{WS}_{2}$ were done similarly to the $\mathrm{MoS}_{2}$ procedure and there was no appreciable growth, because of very poor $\mathrm{WO}_{3}$ evaporation. All the samples were further characterized by AFM, Raman, and photoluminescence spectroscopy showing the best crystalline quality and direct bandgap in all.

We introduced a new technique of low-temperature atmospheric pressure CVD synthesis of monolayers of $\mathrm{MoS}_{2}$. We have grown single crystals and polycrystal films using sodium as catalytic. From DFT calculations and experiments, we found the ideal synthesis parameters of precursor ratio 0.3 and synthesis temperature of $550^{\circ} \mathrm{C}$. The DFT calculation also shows that the critical element is the number of sodium in the system, which reduces the 
energy formation and allows to use a reduced temperature $\left(\Delta T=250^{\circ} \mathrm{C}\right)$. Single and polycrystalline monolayers can be achieved by controlling the growth time. The XPS spectra after the bath show that the sodium could not be found in the crystal lattice. The AFM, Raman, and photoluminescence spectroscopy has established it as a monolayer with a direct bandgap and has a low density of defects. The optical and electrical experiments show that the grown samples have properties comparable to those produced at a higher temperature at any arbitrary substrate. The new technique will help to synthesized directly on a transparent substrate, allowing the low-cost and high-quality synthesis of these semiconducting material. This work would serve as a reference for the low-temperature synthesis of other TMDs. 


\section{Second Harmonic Generation in Two and Three Layers of Transition Metal Dichalcogenides}

This chapter includes the characterization of two and three layers of TMDs having different crystal orientations. The numbers of layers were confirmed by Raman and PL spectroscopies. Finally, the non-linear optical properties (second and third harmonic generation) of two and three layers $\mathrm{MoS}_{2}$, $\mathrm{WS}_{2}$, and $\mathrm{MoS}_{2} / \mathrm{WS}_{2}$ samples. The second harmonic shows that the layers grow with different crystal orientations $\left(0^{\circ}\right.$ or $\left.60^{\circ}\right)$ have different phase stacking.

\section{1}

\section{Introduction}

After the consistent growth of monolayer triangular islands, the next challenging task was to grow uniform layered triangles. It can be seen in figure 4.1(a) the triangles formed had a very clear nucleation site visible in the centre of the triangle and even some with growth occurring off that nucleation site presenting the beginning of a second layer. The second layer is somehow easy to grow but difficult to control. We report the atmospheric pressure chemical vapor deposition synthesis of two and three layers of $\mathrm{MoS}_{2}$ and $\mathrm{WS}_{2}$. The APCVD synthesis of bilayer TMDs is explained (chapter 3 see section 3.1.4). However, controlling the growth of bilayer or multiple layers is still challenging. CVD synthesis of bilayers gives us the samples with different phase stacking between the top and bottom layers i.e $0^{\circ}$ or $60^{\circ}$ (see figure 5.1). The stacking sequence in TMDs can significantly impact on their electrical and optical properties due to its effect on the electronic band structure [91]. Second harmonic generation is a useful technique to study the stacking sequences [62]. We use second and third harmonic generation to characterize our bilayers and trilayers samples.

\section{2}

\section{Two and Three Layers of Transition Metal Dichalcogenides}

The optical images of the as-grown bilayer and trilayer $\mathrm{MoS}_{2}, \mathrm{WS}_{2}$, and their vertical heterostructures are shown in figure 5.1. In figure 5.1(a,c) we have three layers which make these samples more interesting. Figures 5.1(a,b) show 
(a)

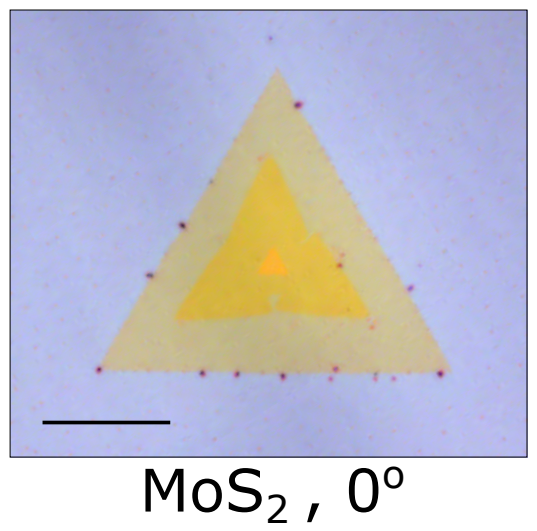

(c)

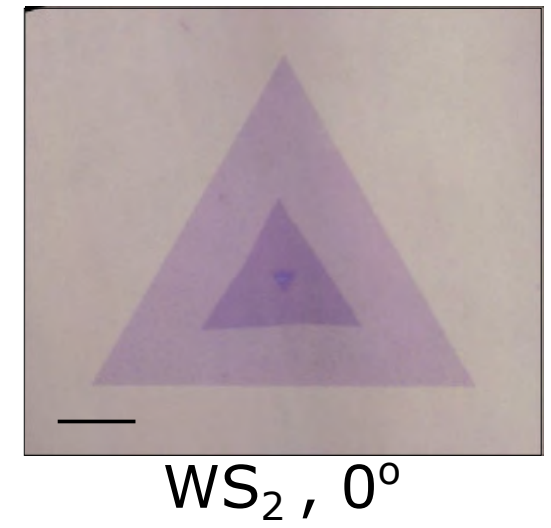

(e)

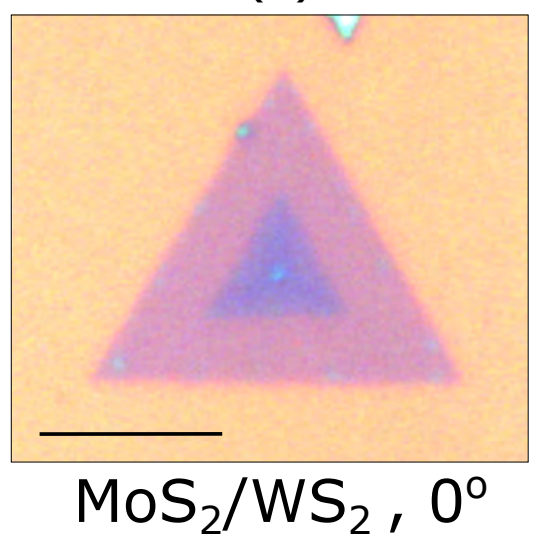

(b)

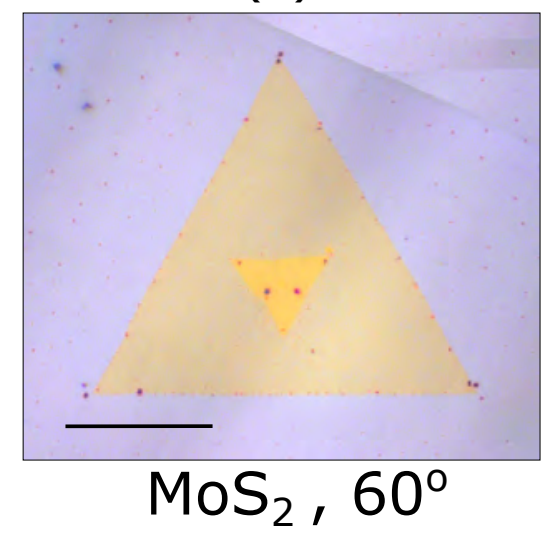

(d)

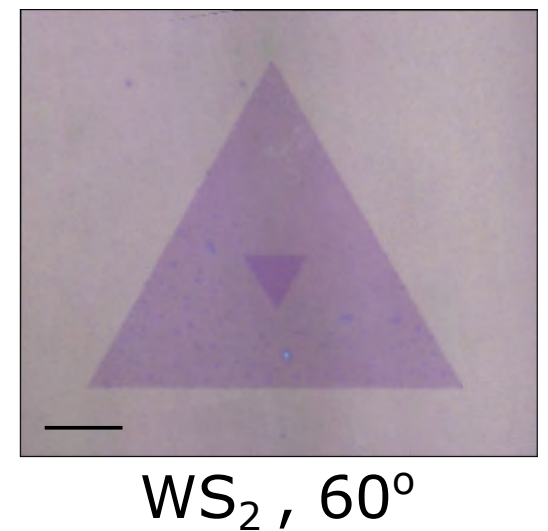

(f)

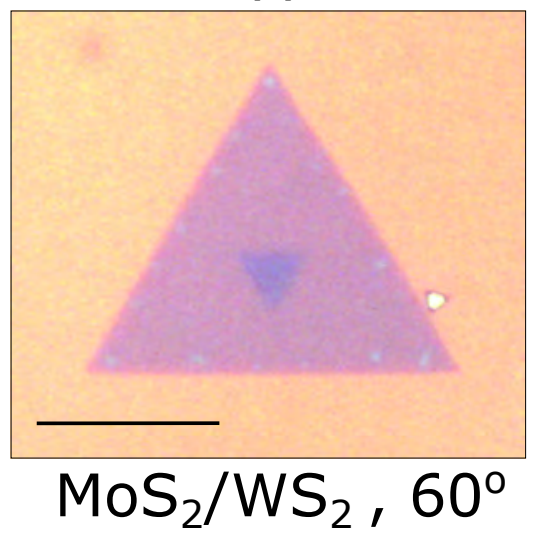

Figure 5.1: Optical microscopy image of the as-grown (a) $\mathrm{MoS}_{2}$ trilayer stacked with same crystal orientation between each layer scale bar is $20 \mu \mathrm{m}$ (b) $\mathrm{MoS}_{2}$ bilayer stacked with opposite crystal orientation to the monolayer (c) $\mathrm{WS}_{2}$ trilayer first two triangles are stacked with same crystal orientation while the third triangle is stacked with $60^{\circ}$ w.r.t other layers scale bar is $10 \mu \mathrm{m}$ (d) $\mathrm{WS}_{2}$ bilayer stacked with opposite crystal orientation (e) $\mathrm{MoS}_{2} / \mathrm{WS}_{2}$ heterostructure stacked at $0^{\circ}$ and (f) $60^{\circ}$, scale bar is $10 \mu \mathrm{m}$.

the optical images of the as-grown trilayer and bilayer $\mathrm{MoS}_{2}$ crystals. Figure 5.1(a) shows a trilayer crystal with the $0^{\circ}$ crystal orientation. The second and third layers are at the centre, and they are smaller than the first layer. Figure 
5.1(b) present a stacked bilayer with a twisted angle of $60^{\circ}$, on which the larger triangle refers to the monolayer, and the triangle inside it is a bilayer region. Figures $5.1(\mathrm{c}, \mathrm{d})$ show the optical images of $\mathrm{WS}_{2}$ trilayer and bilayer crystals. In figure 5.1(c) shows a trilayer crystal with the first two layers have $0^{\circ}$ crystal orientation while the second and the third layers are $60^{\circ}$ oriented. In figure 5.1(e,f) we have as-grown $\mathrm{MoS}_{2} / \mathrm{WS}_{2}$ heterostructures with $0^{\circ}$ and $60^{\circ}$ orientation. The different colour contrast arises due to the difference of $\mathrm{SiO}_{2}$ thickness on the Si substrate [182]. These samples were grown over a period of time, there is no specific reason for different substrates, the substrates were chosen randomly depending on easily available substrate in our laboratory. The $\mathrm{SiO}_{2}$ thickness does not affect the growth process, it only contributes while the characterizations of the sample. For example, Raman intensity strongly depends on the oxide thickness so as the SHG intensity $[183,184]$.

\section{3}

\section{Raman and Photoluminescence of Trilayer Samples}

Figure 5.2(a) depicts the Raman spectrum obtained from $\mathrm{MoS}_{2}$ triangle shown in figure 5.1(a). The recorded Raman spectrum exhibited the characteristic in-plane phonon mode $\mathrm{E}_{2 g}^{1}$ and the out-of-plane $\mathrm{A}_{1 g}$ mode. As the triangle thickness is increased the frequency of the $\mathrm{E}_{2 g}^{1}$ mode decreases (redshifts) while the $\mathrm{A}_{1 g}$ increases (blueshifts). The difference in peak position between these modes in the largest triangle is $20 \mathrm{~cm}^{-1}$ corresponds to monolayer sample [166]. The frequency difference for the middle triangle is $23 \mathrm{~cm}^{-1}$ which corresponds to bilayer, and for the smallest triangle, it is $24.8 \mathrm{~cm}^{-1}$ which corresponds to trilayer [185]. This frequency-dependence shift can identify the ultrathin $\mathrm{MoS}_{2}$ thickness for monolayer to four layers [44]. The photoluminescence of the three layers is also given in figure 5.2(b). The monolayer PL spectrum exhibits a single emission peak, with a maximum at a photon energy of $\sim 1.86$ $\mathrm{eV}$. By increasing the number of layers, the PL intensity decreases a lot, and the maximum of the PL shifts significantly to low energy.

Figure 5.3(a) presents the Raman spectra for one, two, and three-layers of $\mathrm{WS}_{2}$ triangle shown in figure 5.1(c). The intensity ratio of $\mathrm{LA} / \mathrm{A}_{1 g}$ and peak position difference can be used to identify the number of layers [45]. The most substantial ratio indicates the monolayer. Moreover, the first order in-plane mode $\mathrm{E}_{2 g}^{1}$ shows redshifts and the out-of-plane $\mathrm{A}_{1 g}$ shows blueshifts while increasing the number of layers. The difference in peak position between them in the largest triangle is $61.1 \mathrm{~cm}^{-1}$, for the middle triangle is 63.2 $\mathrm{cm}^{-1}$, and for the smallest triangle, it is $64 \mathrm{~cm}^{-1}$ which is the confirmation of monolayer, bilayer and trilayer regions $[45,186]$. Figure $5.3(\mathrm{~b})$ shows the 
(a)

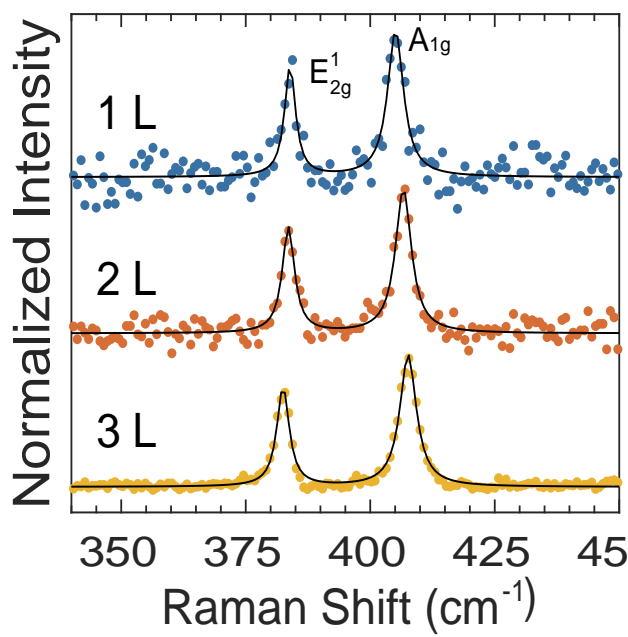

(b)

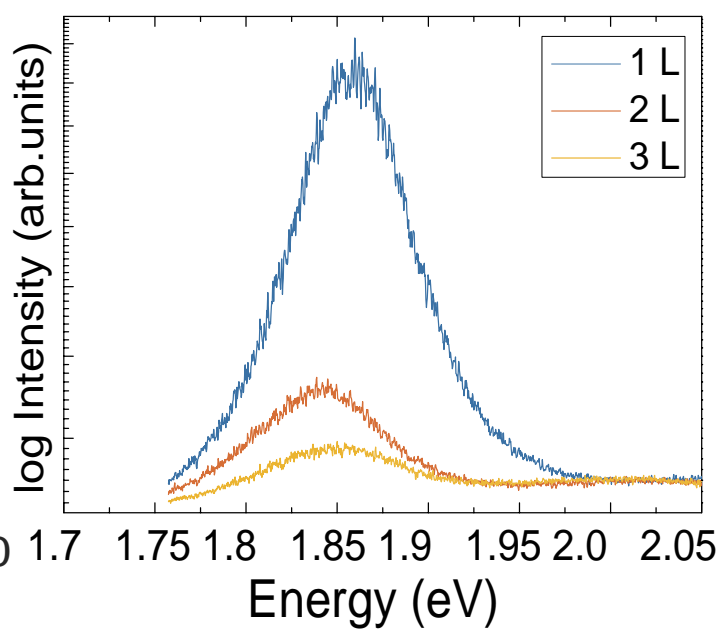

Figure 5.2: (a) Raman spectra of $\mathrm{MoS}_{2}$ trilayer stacked with same crystal orientation between each layer shown in figure 5.1(a). (b) Log of photoluminescence intensity of the three layers, decrease in intensity refers to shifting towards indirect band gap.

photoluminescence from the three layers. The PL shows a sharp peak at $\sim 2.02$ $\mathrm{eV}$ for the biggest triangle i.e monolayer. As the number of layers increases the PL shifts to lower energies and the intensity decreases drastically [187]. This layer dependent photoluminescence in $\mathrm{MoS}_{2}$ and $\mathrm{WS}_{2}$ is a result of direct-toindirect transition [105].

(a)

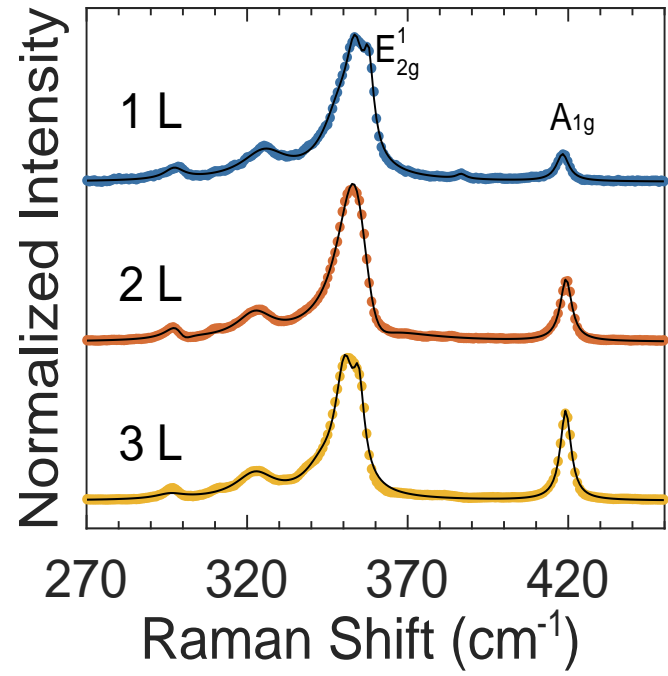

(b)

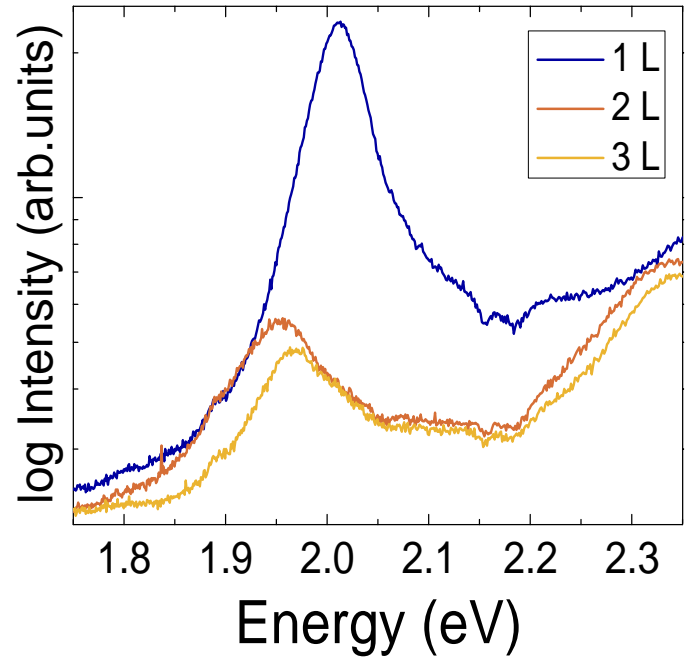

Figure 5.3: (a) Raman spectra of $\mathrm{WS}_{2}$ trilayer shown in figure 5.1(c). (b) Log of photoluminescence intensity of the three layers. 
Direct bandgap in $\mathrm{MoS}_{2}$ and $\mathrm{WS}_{2}$ depends on the localized d-orbital of the $\mathrm{Mo} / \mathrm{W}$ atom. Which are not much affected by the inter-layer coupling due to its location in the unit cell. While indirect bandgap in these materials depends on the overlap of d-orbital of the Mo/W atom and $\mathrm{p}_{z}$ orbital of the $\mathrm{S}$ atoms which strongly depends on inter layer coupling. Thus, as the number of layers is decreased the intrinsic direct bandgap of the material becomes more pronounced. While the band gap broadening in the monolayer is attributed to quantum confinement of charge carriers, common in nanosystems [104].

$4 \mathrm{~L}$

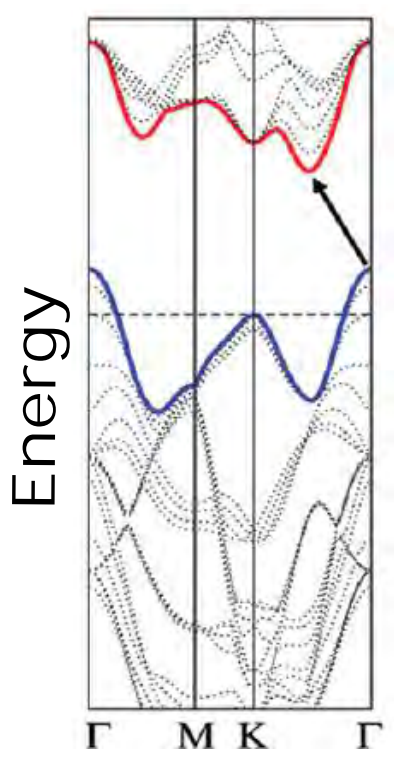

$2 \mathrm{~L}$

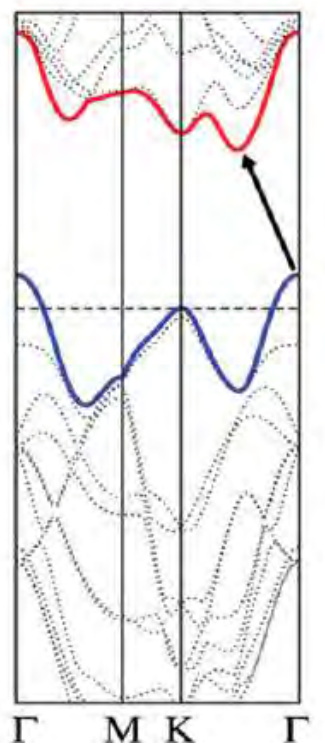

$1 \mathrm{~L}$

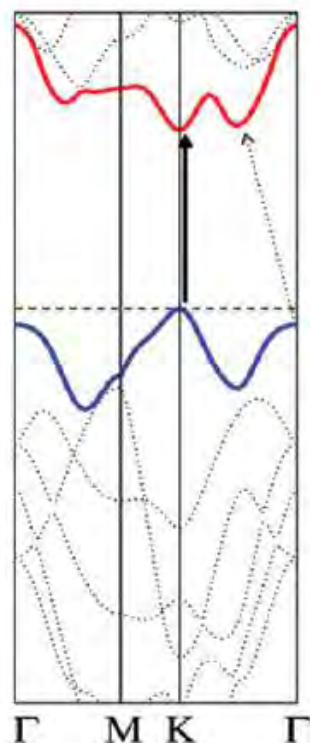

Figure 5.4: Band gap of 4L, 2L, and 1L of $\mathrm{MoS}_{2}$ calculated by Splandani et al. [9]. The solid arrows indicate the lowest energy transitions. The direct excitonic transitions occur at high energies at $\mathbf{K}$ point. With reduced layer thickness, the indirect bandgap becomes larger, while the direct excitonic transition barely changes. For monolayer $\mathrm{MoS}_{2}$, it becomes a direct bandgap semiconductor. This dramatic change of electronic structure in monolayer $\mathrm{MoS}_{2}$ can explain the observed jump in monolayer photoluminescence efficiency.

This transition bandgap behaviour was explained by Splandani et al. [11] They did the DFT calculations for few layers $\mathrm{MoS}_{2}$ see figure 5.4. It can be seen that the direct excitonic transition energy at $\mathbf{K}$ point in the Brillouin zone hardly changes with layer number. But the indirect bandgap see increasing in energy as the number of layers decreases. The indirect transition energy becomes so high in monolayer $\mathrm{MoS}_{2}$ and it eventually becomes a 2-D direct bandgap semiconductor. With the increase of the indirect bandgap in thinner $\mathrm{MoS}_{2}$, the intraband relaxation rate from the excitonic states decreases, and the photoluminescence becomes stronger. 
(a)

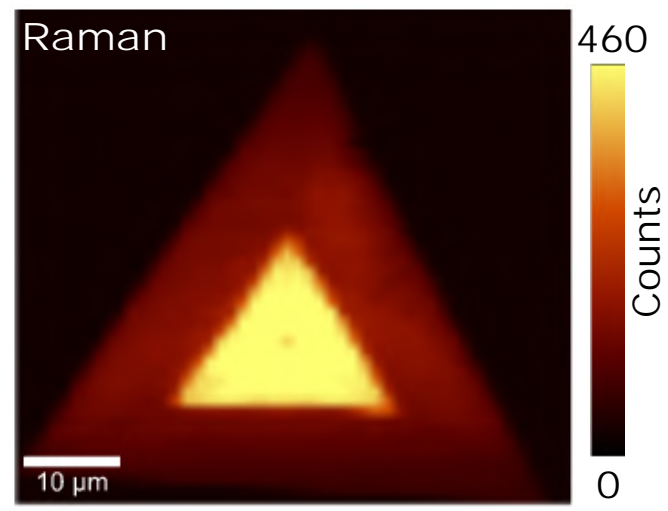

(b)

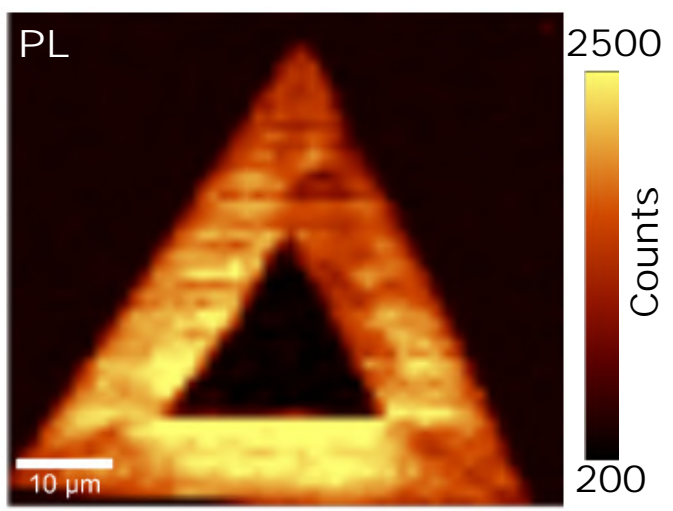

Figure 5.5: (a) Raman and (b) PL mapping images of the $\mathrm{WS}_{2}$ trilayer shown in figure 5.1(c).

The Raman and PL maps of the $\mathrm{WS}_{2}$ triangular flake (shown in figure 5.1(c)) are also given in figure 5.5(a). It can be seen the Raman intensity is uniform over the area and in the bilayer region the intensity increases, while there is a minor change in the intensity of bilayer and trilayer regions. The PL intensity map of the same triangle is given in figure 5.5(b). The PL intensity decreases at the bilayer, compared to monolayer as expected. The Raman and $\mathrm{PL}$ of $\mathrm{MoS}_{2} / \mathrm{WS}_{2}$ heterostructure has already been discussed in chapter 4 (see section 4.3).

\section{4}

Second Harmonic Generation in Two and Three Layers of $\mathrm{MoS}_{2}, \mathrm{WS}_{2}$ with Different Stacking Orientation

Three sets of bi-layer samples $\mathrm{MoS}_{2} 0^{\circ}$ and $60^{\circ}, \mathrm{WS}_{2} 0^{\circ}$ and $60^{\circ}$ and $\mathrm{MoS}_{2} / \mathrm{WS}_{2} 0^{\circ}$ and $60^{\circ}$ (see figure 5.1) were chosen for nonlinear optical characterization (SHG and THG). The $1560 \mathrm{~nm}$ Erbium-doped fiber laser with a pulse duration of 150 fs was used as an incident laser, experimental setup has been discussed in chapter 3 (see section 3.8).

Figure 5.6 shows spectra from $\mathrm{MoS}_{2}$ flake in figure 5.1(a) which depicts prominent peaks at $\sim 780 \mathrm{~nm}$, which represents the exact frequency doubling signal (SHG) from the $1560 \mathrm{~nm}$ (Erbium-doped fiber laser). This indicates that the signals are generated through a second-order nonlinear process in $\mathrm{MoS}_{2}$ flake. Similarly, peaks at $\sim 520 \mathrm{~nm}$ indicate third-harmonic signals are observed from $\mathrm{MoS}_{2}$ flake in figure 5.1(a). The second harmonic generated signals from the three regions of $\mathrm{MoS}_{2}$ flake show the monolayer has the lowest intensity and trilayer has the highest intensity. The SHG signal from the monolayer area 


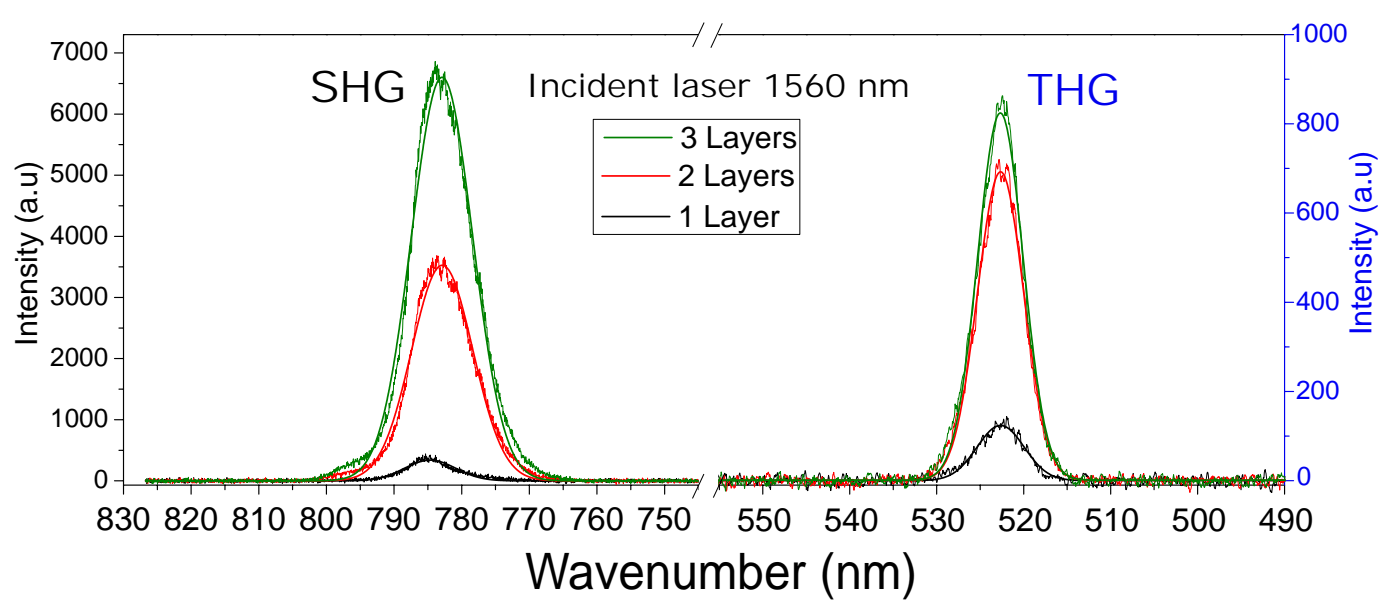

Figure 5.6: Single spectra of $\mathrm{MoS}_{2}$ flake shown in figure 5.1(a), showing secondharmonic and third-harmonic signals.

of $60^{\circ}$ sample (figure $5.1(\mathrm{~b})$ ) is equal monolayer area of $0^{\circ}$ sample. However, the SHG is not observed in bilayer area of $60^{\circ}$ sample. Similar trend is followed by the third harmonic generation by trilayer sample but the THG signal is very weak as compared to SHG. However, THG is observed in bilayer area of $60^{\circ}$ sample.

The polarization dependence of the SHG signals was also studied. Polarization angle $\theta$ is defined as the angle of the electric field direction of the incident laser with respect to the long axis of the sample. SHG is very sensitive to the crystal symmetry. Hence, polarization resolved SHG measurements provide important crystallographic information of TMDs atomic layers [188].

(a) $\mathrm{MoS}_{2} 0^{\circ}$

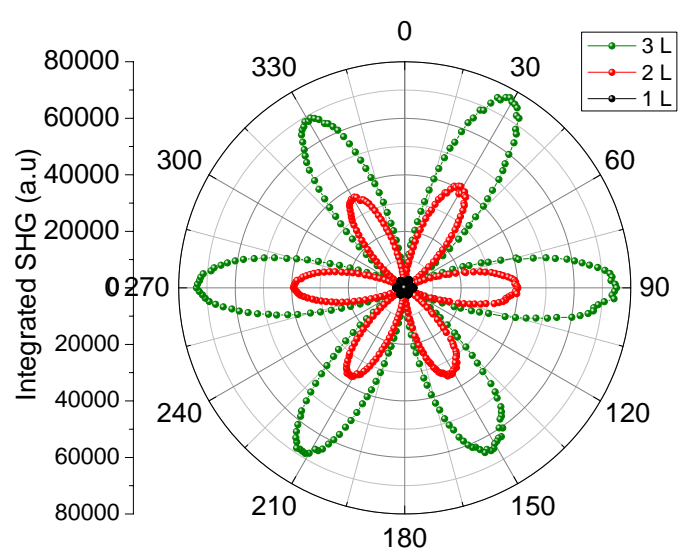

(b) $\mathrm{MoS}_{2} 60^{\circ}$

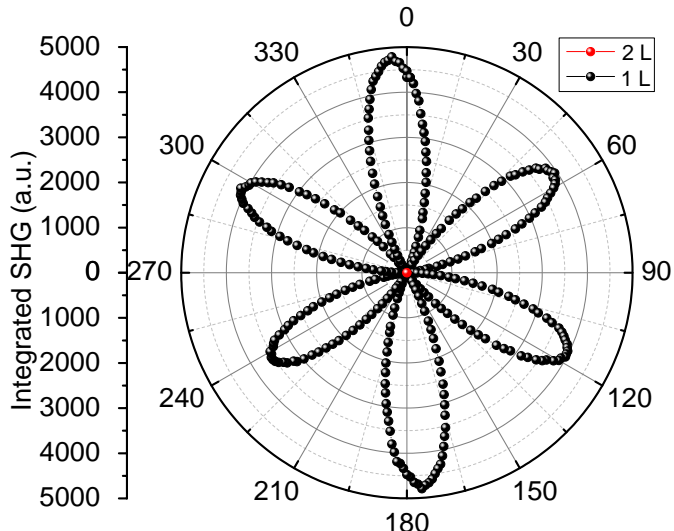

Figure 5.7: Intensity dependence of the SHG signal with the polarization angle. (a) Trilayer (green) with a twisted angle of $0^{\circ}$ between bilayer (red) and monolayer (black). (b) bilayer (red) twisted $60^{\circ}$ from monolayer (black). 
Second harmonic generation was measured at polarization angles from $0^{\circ}$ to $360^{\circ}$ with a step of $1^{\circ}$. Each spectrum was fitted with a gaussian and intensity is estimated. Figure 5.7(a,b) present the SHG intensity as a function of the polarization angle, obtained from crystals in figure 5.1(a,b). The polar graphs show the polarization dependence with the six-fold pattern.

(a)

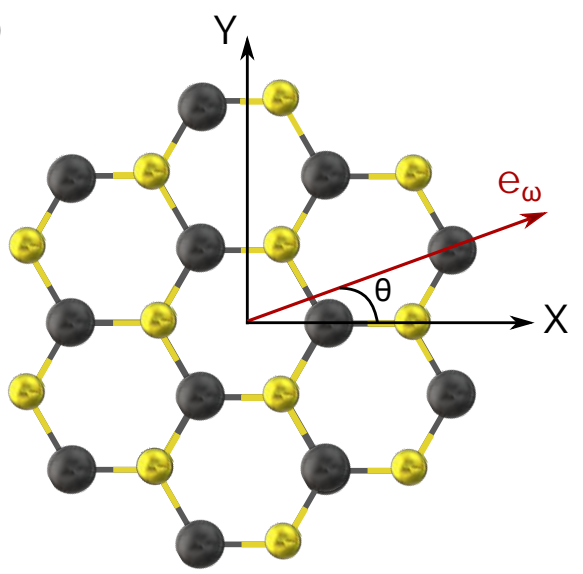

(b)

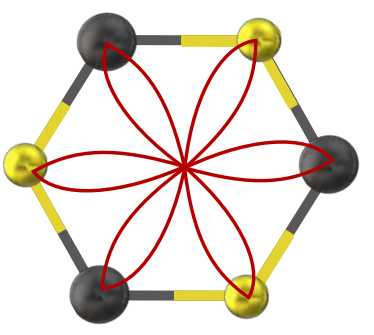

Figure 5.8: Schematics of (a) Top view of the $\mathrm{MoS}_{2}$ crystallographic orientation with respect to the incident laser polarization $\mathrm{e}_{\omega}$. (b) Orientational dependence of SHG.

The flower-like six petals polarization dependence is due to the $\mathrm{D}_{3 h}$ symmetry of the $\mathrm{MoS}_{2}$ monolayer. Since $\mathrm{MoS}_{2}$ has hexagonal crystal symmetry, the electron cloud oscillates (due to external electric field) as a function of angle $\theta$. Hence, generating a graph with periodicity of $60^{\circ}$. Figure 5.8 is a sketch of a top view of hexagonal $\mathrm{MoS}_{2}$, showing the angle of the crystallographic orientation to the incident laser polarization direction $\mathrm{e}_{\omega}[51,189]$.

$\mathrm{D}_{3 h}$ symmetry group gives only one independent nonvanishing element of the nonlinear response i.e:

$$
\chi^{(2)}=\chi_{x x x}^{(2)}=-\chi_{x y y}^{(2)}=-\chi_{y y x}^{(2)}=-\chi_{y x y}^{(2)}
$$

where $\mathrm{x}$ corresponds to the armchair direction and $\mathrm{y}$ is the zigzag direction (see figure 5.8). The electric field of the generated second harmonic signal $\mathrm{E}(2 \omega)$ along a given direction $e_{2 \omega}$ can be described in terms of $\chi^{(2)}$ and input light polarization vector $\mathrm{e}_{\omega}$ as:

$$
E(2 \omega) \cdot e_{2 \omega}=C e_{2 \omega} \cdot \chi^{(2)}: e_{\omega} e_{\omega}
$$

here $2 \omega$ is the second harmonic frequency, $\omega$ is the incident laser frequency, and $\mathrm{C}$ is constant (local-field factors determined by the local dielectric environment). For a laser polarization perpendicular or parallel to the polarization of the input laser, the generated second-harmonic electric field is expressed as $[50,190]$ : 


$$
E_{\perp}(2 \omega)=C \chi^{(2)} \sin \left(3 \theta+\theta_{o}\right)
$$

or

$$
E_{\|}(2 \omega)=C \chi^{(2)} \cos \left(3 \theta+\theta_{o}\right)
$$

where $\theta$ is the angle between the input laser polarization and the $\mathrm{x}$ direction, $\theta_{o}$ is the initial crystallographic orientation of the sample. Since intensity is proportional to the square of the electric field $\left(\mathrm{I}_{n t} \propto|E|^{2}\right)$. Therefore, the intensity of the generated SHG is proportional to $\cos ^{2}\left(3 \theta+\theta_{o}\right)$ or $\sin ^{2}(3 \theta+$ $\theta_{o}$ ), giving six-fold polarization dependence.

By obtaining the polar plots it is possible to analyze the relationship of intensity of the layers and the phase angle between them.

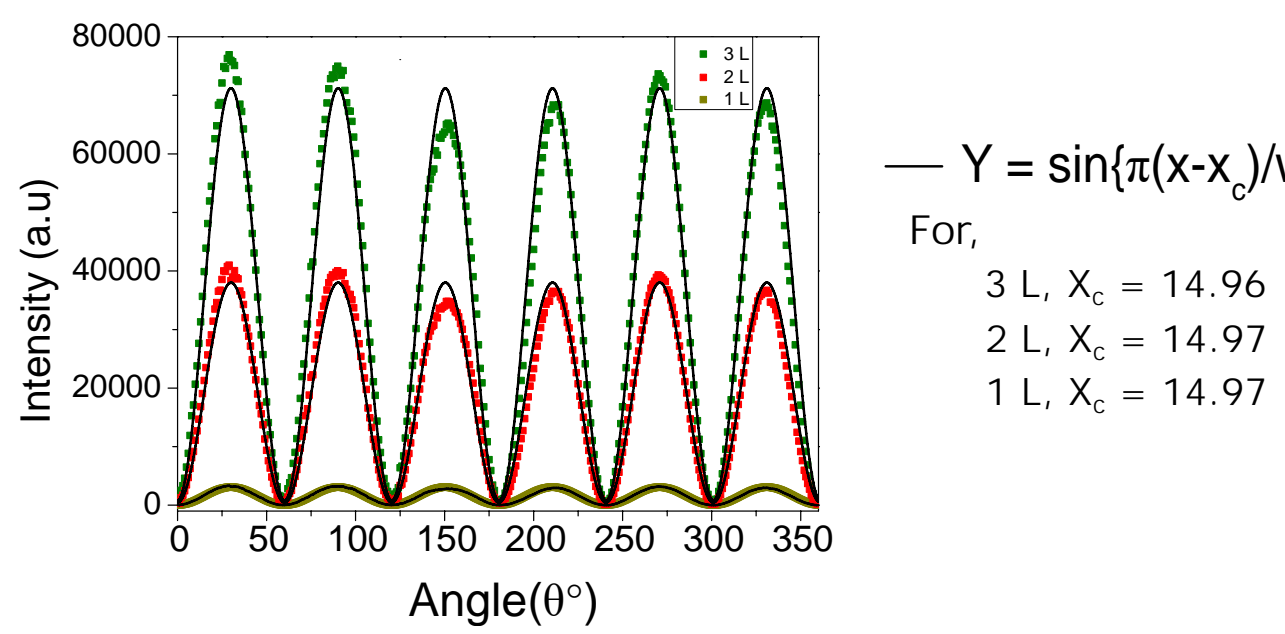

Figure 5.9: Polarization plot shown in figure 5.7(a), fitted with a sine wave function (black line) showing no phase difference between each layer.

Each polarization plot was fitted using a sine function with a period of $60^{\circ}$. Figure 5.9 presents the polarization plot shown in figure 5.7(a) along with the fitted sine wave function. These fittings gives us the phase difference between each layer. Table 5.1 shows the SHG intensity normalized by the monolayer and phase difference for two and three stacking $\mathrm{MoS}_{2}$ layers with $0^{\circ}$ and $60^{\circ}$ twisted angle. We observed an enhancement of SHG intensity, for the bilayer with $0^{\circ}$, the value is $\sim 12$ times, and for trilayer is $\sim 23$ times higher than the monolayer intensity. The observed SHG increase is around four times higher than expected, most likely due to the perfect stacking of two layers in CVD growth [62]. For the $60^{\circ}$ twisted angle, the SHG signal at the bilayer region is inhibited due to inversion-symmetry. The phase near $0^{\circ}$ shows that the layers are aligned. Figures $5.10(\mathrm{a}, \mathrm{b})$ show the schematics of relative stacking orientation of the top and bottom layers. In the $3 \mathrm{R}(\mathrm{AB})$ phase, our $0^{\circ}$ bilayer and trilayer $\mathrm{MoS}_{2}$, the metal atom is located at the centre of the bottom 
hexagon. Thus we have a no-inversion symmetry and the SHG is present. In the $2 \mathrm{H}\left(\mathrm{AA}^{\prime}\right)$ phase, our $60^{\circ}$ bilayer $\mathrm{MoS}_{2}$, the stacking of metal atom of the second layer sits precisely on top of the chalcogen atom of the bottom layer and shows the inversion symmetry and therefore no SHG signal [88,94]. The simple approach can explain qualitatively the absence of second-harmonic generation in $60^{\circ}$ bilayer $\mathrm{MoS}_{2}$, due to the presence of inversion symmetry the $\chi_{2}$ tensor is equal to zero.

(a)

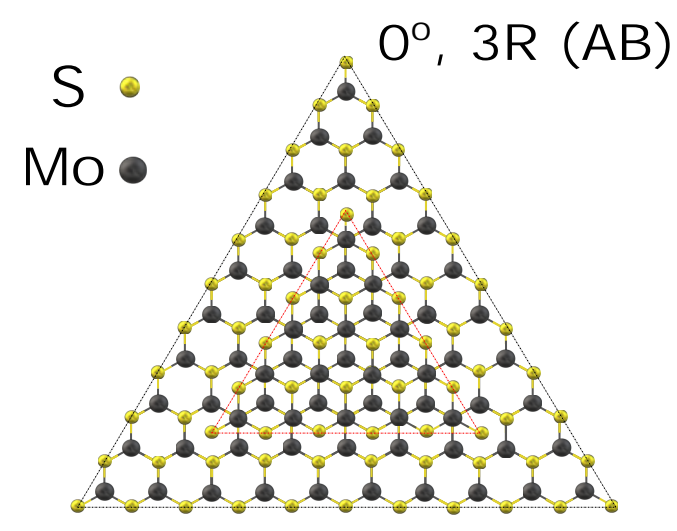

(b)

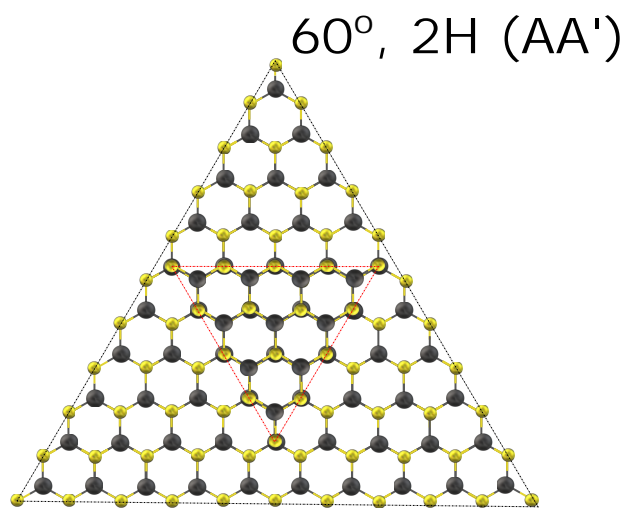

Figure 5.10: Schematics of relative stacking orientation of the top and bottom layers. (a) $3 \mathrm{R}(\mathrm{AB})$ phase, the metal atom is located at the center of the bottom hexagon. Thus has a no-inversion symmetry. (b) $2 \mathrm{H}\left(\mathrm{AA}^{\prime}\right)$ phase, the metal atom of the second layer sits precisely on top of the chalcogen atom of the bottom layer, has the inversion symmetry.

Generally, even number of $\mathrm{MoS}_{2}$ layers is known to have no SHG (even number of layers have inversion symmetry) [122], but it can be generated in an odd number of $\mathrm{MoS}_{2}$ layers (no-inversion symmetry) [50-52]. Here, we have shown that CVD grown bilayers of $\mathrm{MoS}_{2}$ could possess two types of stacking (i.e $2 \mathrm{H}$ and $3 \mathrm{R}$ ) and could have inversion or no-inversion symmetries.

The polarization dependent third harmonic generation (THG) for these flakes were also studied. Third harmonic generation was measured at polarization angles from $0^{\circ}$ to $360^{\circ}$ with a step of $5^{\circ}$. In contrast to SHG, the THG is polarization independent, as can be seen from figures 5.11(a,b). The THG intensity increases with the number of layers [124]. As the thicker sample has longer light-matter interaction length. Also, we have THG in bilayer $\mathrm{MoS}_{2} 60^{\circ}$ sample, as it is not tied to the condition of non-centrosymmetry. 
(a) $\mathrm{MoS}_{2} 0^{\circ}$

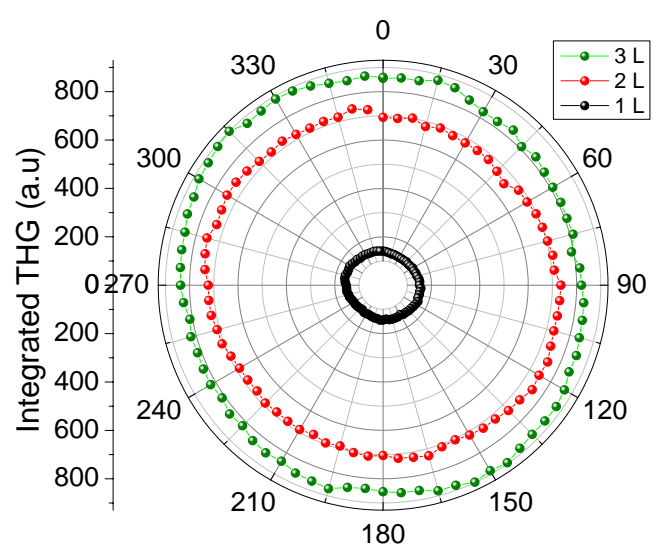

(b) $\mathrm{MoS}_{2} 60^{\circ}$

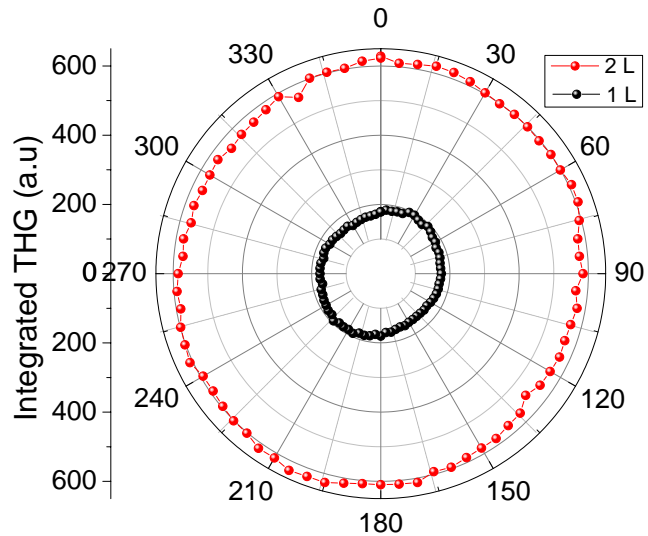

Figure 5.11: THG, constant intensity with $\theta$ (a) Trilayer $\mathrm{MoS}_{2}$ shown in figure 5.1(a) (b) Bilayer $\mathrm{MoS}_{2}$ shown in figure 5.1(b).

Figure 5.12(a,b) present the polarization plots of the nonlinear optical response for $\mathrm{WS}_{2}$ trilayers and bilayers crystals shown in figure 5.1(c,d). We observed, once more, the six-fold pattern. The bilayers with $0^{\circ}$ and $60^{\circ}$ show similar behaviour compared with $\mathrm{MoS}_{2}$ crystals. However, the top layer of the trilayer crystal is rotated $60^{\circ}$. The measurements also provided the possibility to verify the phase difference of the signal in each region, which could be caused by rotation between the layers.

(a) $\mathrm{WS}_{2} 0^{\circ} \& 60^{\circ}$

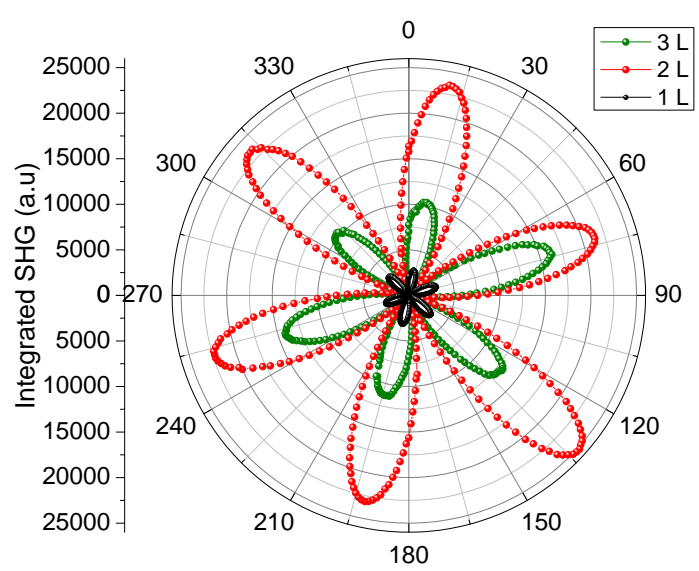

(b) $\mathrm{WS}_{2} 60^{\circ}$

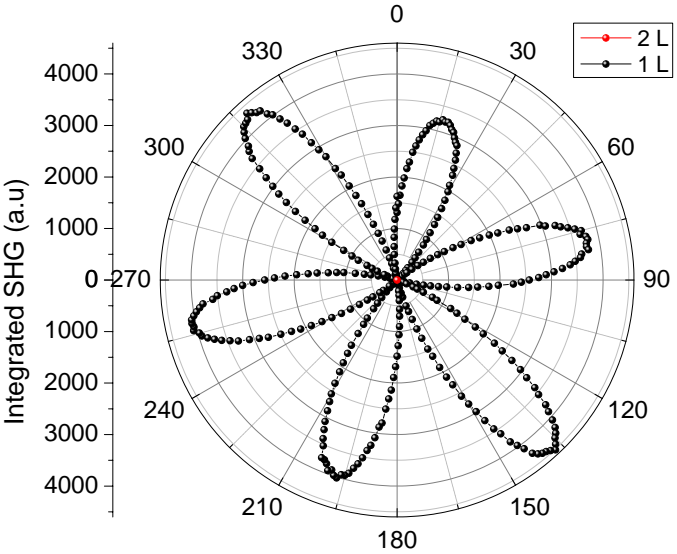

Figure 5.12: Intensity dependence of the SHG signal with the polarization angle. (a) Trilayer (green) with twisted angle of $0^{\circ}$ between bilayer(red) and monolayer (black). (b) bilayer (red) twisted $60^{\circ}$ from monolayer (black). 
of $\mathrm{WS}_{2}$. For bilayers with a twisted angle at $0^{\circ}$, the SHG intensity is $\sim 7.7$ times higher than the monolayer. For the trilayer region, we observed a reduction of SHG intensity, probably due to the inversion of symmetry generated by the top layer. In the crystals with $60^{\circ}$ figure $5.1(\mathrm{~d})$, it is not possible to detect the SHG signal, due to the centrosymmetry, and therefore eliminating secondorder nonlinearities. By fitting the sine wave on the polarization plot in figure 5.12 it is confirmed that the $\mathrm{WS}_{2}$ sample did not show rotation between its layers.

\subsection{1}

\section{SHG in $\mathrm{MoS}_{2} / \mathrm{WS}_{2}$ Heterostructure with Different Stacking Orientation}

(a) $\mathrm{MoS}_{2} \backslash \mathrm{WS} \mathrm{S}_{2} 0^{\circ}$

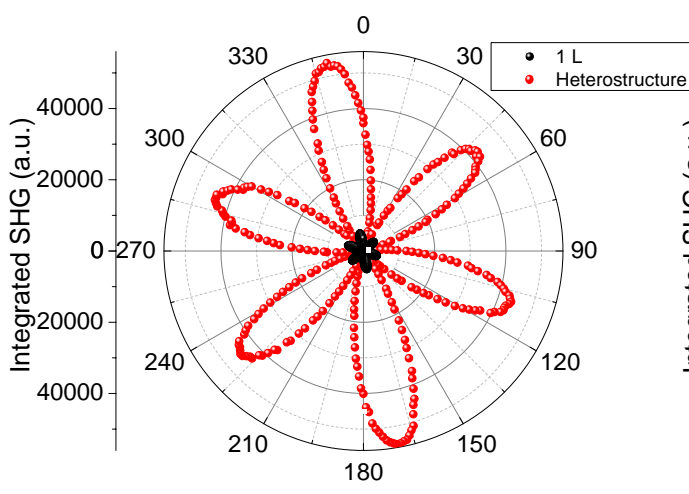

(b) $\mathrm{MoS}_{2} \backslash \mathrm{WS}_{2} 59^{\circ}$

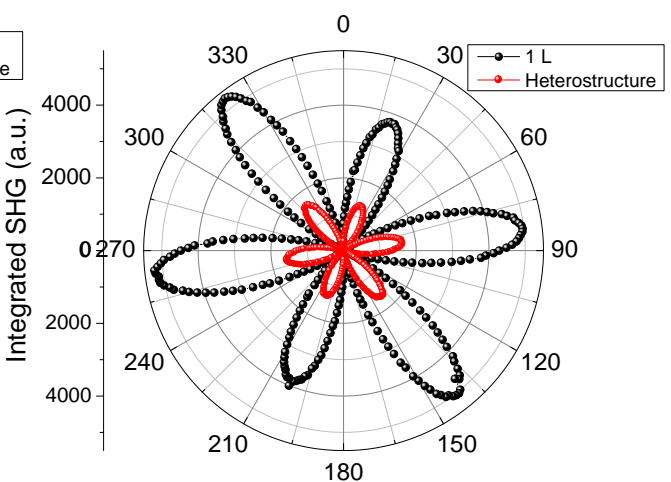

Figure 5.13: Intensity dependence of the SHG signal with the polarization angle. (a) $\mathrm{MoS}_{2} / \mathrm{WS}_{2}$ heterostructure $0^{\circ}$. and (b) $\mathrm{MoS}_{2} / \mathrm{WS}_{2}$ heterostructure $59^{\circ}$.

The SHG from $\mathrm{MoS}_{2} / \mathrm{WS}_{2}$ heterostructure samples (Figure 5.1(e,f)) was also measured. Figure 5.13(a,b) presents the polarization dependence of the SHG signal of heterostructure. The extinct rotational symmetry was observed in both graphs. In addition, the relationship between the intensity of SHG in the heterostructure and the intensity in the monolayer showed SHG signal is $\sim 10$ times higher in bilayer region than the monolayer [191].

In contrast to the same bilayer $\mathrm{MoS}_{2}$ and $\mathrm{WS}_{2} 60^{\circ}$ samples, heterostructure $60^{\circ}$ sample produced a SHG signal but have very low intensity compared to monolayer, see figure 5.13(b). The sine function with a period of $60^{\circ}$ is fitted to calculate the phase difference between each layer. For heterostructure $0^{\circ}$ the phase difference is $0^{\circ}$ between two layers but for $60^{\circ}$ heterostructure the alignment is not exact, there is a phase difference of $\sim 1^{\circ}$. Since the phase difference between the two layers could result in SHG. To confirm our observation we took another heterostructure $60^{\circ}$ crystal with $0^{\circ}$ phase difference and 
measured the polarization dependence of SHG again. Once again we observe SHG (figure 5.14). This is due to the presence of two different materials (i.e Mo and W), the complete cancellation of the SHG signal is not possible (there are not the same materials to generate exact inversion symmetry).

Figures 5.15(a,b) show the schematics of relative stacking orientation of the top and bottom layers of $\mathrm{MoS}_{2} / \mathrm{WS}_{2}$ heterostructure samples. Heterostructure possesses the same $2 \mathrm{H}$ and $3 \mathrm{R}$ phase stacking as bilayer $\mathrm{MoS}_{2}$ and $\mathrm{WS}_{2}$ did, but the different masses of $\mathrm{W}$ atom (top layer) and Mo atom (bottom layer) is responsible for SHG in $2 \mathrm{H}$ stacked heterostructure. This behaviour of SHG can be explained as the interference of two electromagnetic waves.

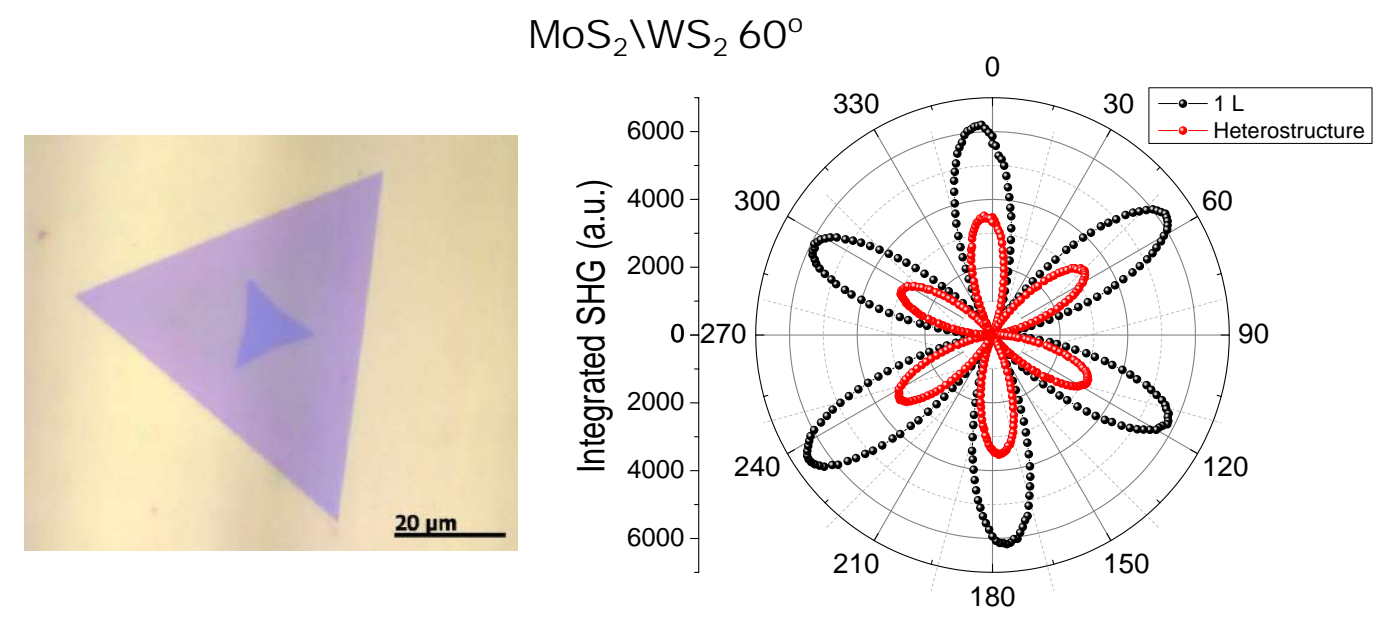

Figure 5.14: Optical image of $\mathrm{MoS}_{2} / \mathrm{WS}_{2}$ heterostructure $60^{\circ}$, and its intensity dependence of the SHG signal with the polarization angle.

(a)

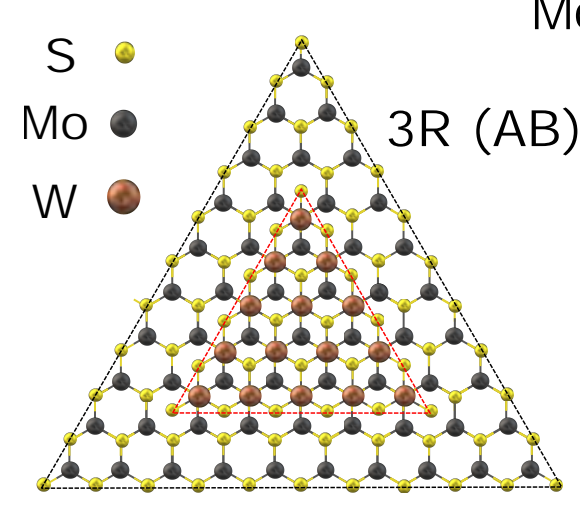

(b)

$\mathrm{MoS}_{2} / \mathrm{WS}_{2}$

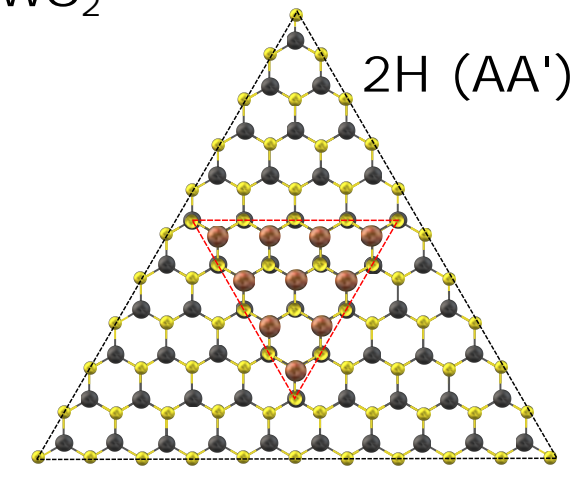

Figure 5.15: Schematics of relative stacking orientation of the top and bottom layers. (a) $\mathrm{MoS}_{2} / \mathrm{WS}_{2} 0^{\circ}$. (b) $\mathrm{MoS}_{2} / \mathrm{WS}_{2} 60^{\circ}$, mismatch between the masses of Mo and $\mathrm{W}$ atoms results in SHG. 


\begin{tabular}{|l||c|c|}
\hline Stacking & $\begin{array}{c}\text { Normalized SHG } \\
\text { Intensity }\end{array}$ & Phase \\
\hline \hline $0^{\circ}$ Bilayer $\mathrm{MoS}_{2}$ & $12.3 \pm 1.2$ & $0.0 \pm 0.2^{\circ}$ \\
\hline $0^{\circ}$ Trilayer $\mathrm{MoS}_{2}$ & $23.1 \pm 2.2$ & $0.0 \pm 0.1^{\circ}$ \\
\hline $0^{\circ}$ Bilayer $\mathrm{WS}_{2}$ & $7.71 \pm 0.07$ & $0.0 \pm 0.1^{\circ}$ \\
\hline $60^{\circ}$ Trilayer $\mathrm{WS}_{2}$ & $5.0 \pm 0.2$ & $0.0 \pm 0.2^{\circ}$ \\
\hline $0^{\circ} \mathrm{MoS}_{2} / \mathrm{WS}_{2}$ & $10.0 \pm 0.01$ & $1.0 \pm 0.2^{\circ}$ \\
\hline $59^{\circ} \mathrm{MoS}_{2} / \mathrm{WS}_{2}$ & $0.34 \pm 0.5$ & $0.0 \pm 0.2^{\circ}$ \\
\hline $60^{\circ} \mathrm{MoS}_{2} / \mathrm{WS}_{2}$ & $0.51 \pm 0.4$ & \\
\hline
\end{tabular}

Table 5.1: Normalized SHG intensity and phase of TMD's.

\section{4 .2}

\section{Interference of Two S.H Fields}

SHG from the two distinct layers can be considered as the superposition of two second harmonic fields, generated from two individual layers. The phase difference between them depends on the stacking angle between these two layers [62]. Consider these layers as electrically decoupled layers. The linearly polarized incident laser generates linearly polarized second harmonic fields from each layer, with a polarization direction determined by the angle between laser polarization and the armchair direction of each layer. If the incident laser electric field $\mathrm{E}(\omega)$ makes an angle of $\theta_{1}$ and $\theta_{2}$ w.r.t the armchair direction of our triangles. Therefore, the generated second harmonic electric field will be $E_{1}(2 \omega)$ and $E_{2}(2 \omega)$ making an angle $3 \theta_{1}$ and $3 \theta_{2}$ from the laser polarization (equation 5-3). The total second harmonic electric field will be (figure 5.16).

$$
E_{T}(2 \omega)=E_{1}(2 \omega)+E_{1}(2 \omega)--- \text { (vector superposition) }
$$

since total intensity $I_{T} \propto\left|E_{T}\right|^{2}$ then squaring 5-5

$$
\left|E_{T}(2 \omega)\right|^{2}=\left(\left|E_{1}(2 \omega)\right|\right)^{2}+\left(\left|E_{2}(2 \omega)\right|\right)^{2}+2\left|E_{1}(2 \omega)\right| .\left|E_{2}(2 \omega)\right|
$$

$$
\left|E_{T}(2 \omega)\right|^{2}=\left|E_{1}(2 \omega)\right|^{2}+\left|E_{2}(2 \omega)\right|^{2}+2 \sqrt{\left|E_{1}(2 \omega)\right|^{2}\left|E_{2}(2 \omega)\right|^{2}} \cdot \cos 3\left(\theta_{1}-\theta_{2}\right)
$$

Replacing $|E|^{2}$ with I

$$
I_{T}=I_{1}+I_{2}+2 \sqrt{I_{1} I_{2}} \cdot \cos 3 \theta
$$


Here $\theta$ is the stacking angle between two layers and $\mathrm{I}_{1}, \mathrm{I}_{2}$ stand for the second harmonic intensity in layer 1 and layer 2;

Case 1 If $\theta=0^{\circ}$ and $\mathrm{I}_{1} \approx \mathrm{I}_{2}$ (each layer is generating S.H with the same intensity), Then from equation 5-8

$$
\begin{aligned}
& \mathrm{I}_{T}=2 \mathrm{I}+2 \mathrm{I}(+1) \\
& \mathrm{I}_{T}=4 \mathrm{I}-\cdots-(\text { constructive interference) }
\end{aligned}
$$

In case of bilayers $\mathrm{MoS}_{2}$ and $\mathrm{WS}_{2}$ with $0^{\circ}$ stacking.

Case 2 If $\theta=60^{\circ}$ and $\mathrm{I}_{1} \approx \mathrm{I}_{2}$,

Then from equation 5-8

$$
\begin{aligned}
& \mathrm{I}_{T}=2 \mathrm{I}+2 \mathrm{I}(-1) \\
& \mathrm{I}_{T}=0---- \text { (destructive interference) }
\end{aligned}
$$

In case of bilayers $\mathrm{MoS}_{2}$ and $\mathrm{WS}_{2} 60^{\circ}$ stacking.

Case 3 If $\theta=60^{\circ}$ and $\mathrm{I}_{1} \neq \mathrm{I}_{2}$ (each layer is generating S.H with different intensities i.e when we have two different layers),

Then from equation 5-8, we must have SHG intensity.

That is the case of $\mathrm{MoS}_{2} / \mathrm{WS}_{2} 60^{\circ}$ sample.

Also at any arbitrary angle except $60^{\circ}$ the two layers will show SHG.

(a)

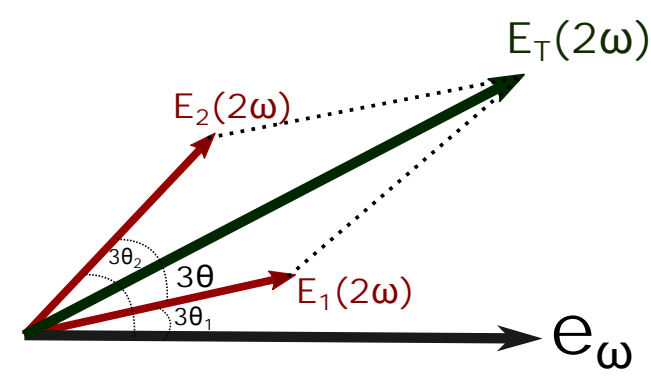

(b)

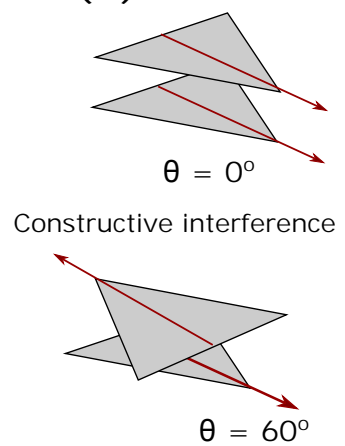

Destructive interference

Figure 5.16: Schematic for illustrating the vector superposition of the second harmonic fields.

\section{5}

\section{Partial Conclusion}

We have grown a few layers of $\mathrm{MoS}_{2}$ and $\mathrm{WS}_{2}$ using chemical vapor deposition. The bilayer and trilayer crystals have $0^{\circ}$ and $60^{\circ}$ of the twisted angle. We used Raman and PL spectroscopies to characterized the crystals. 
The optical properties of these materials confirmed their crystalline structure and thickness. The second harmonic generation was used to study the nonlinear properties of these layers. The SHG shows that the layers in bilayers grown with $0^{\circ}$ have no inversion symmetry refers to $3 \mathrm{R}(\mathrm{AB})$ phase stacking. For $60^{\circ}$, we have an inversion symmetry (Centrosymmetric) and $2 \mathrm{H}\left(\mathrm{AA}^{\prime}\right)$ phase stacking. These studies show that for CVD grown bilayer samples we can achieve both $2 \mathrm{H}\left(\mathrm{AA}^{\prime}\right)$ and $3 \mathrm{R}(\mathrm{AB})$ phases. Moreover, the SHG from tri-layer $\mathrm{MoS}_{2}$ and $\mathrm{WS}_{2}$ crystals confirms our claim. The SHG keeps enhancing when all three layers are $0^{\circ}$ stacked which referred to $\mathrm{ABA}$ stacking, if the third layer is replaced with $60^{\circ}$ stacking concerning to the other two layers the SHG signal enhances in first two layers while blocked on the third layer, this referred to ABA' stacking. SHG is also observed in $\mathrm{MoS}_{2} / \mathrm{WS}_{2} 60^{\circ}(2 \mathrm{H}$ stacking) due to difference of Mo and $\mathrm{W}$ masses. Such behaviour is explained as the interference of two electromagnetic waves. 


\section{6 \\ Electrical Measurements}

This chapter is devoted to the electrical performances of our CVD grown TMDs. Electrical performances were measured by fabricating two probe devices on them. It starts with the introduction and working of conventional field-effect transistors (FETs), including their working mechanisms and characteristics. This is then followed by the electrical measurements of our devices. Finally, devices were characterized by electro-Raman and the dependence of vibrational modes on gate voltage was studied.

\section{1}

\section{Introduction}

While studying the electro-optical properties of semiconducting TMDs, one question arises how the electro-optical properties of these materials change with the Fermi level. The Fermi level of TMDs can be tuned by applying a voltage across the material. This technique was used in order to tune the Fermi level of graphene [192-196]. The influence of the Fermi level, or carrier densities, on the optical properties of TMDs is also very important. In $\mathrm{MoS}_{2}$ top gate field effect transistor configuration, Raman spectroscopy results show the dependence of vibrational mode with respect to the electron doping [81]. However, not much is known about the doping effect on vibrational modes of other TMDs. The influence of carrier density on the photoluminescence of monolayer $\mathrm{MoS}_{2}$ was also observed [116]. TMDs based metal-oxide field effect transistors (MOSFETs) were deeply studied during the past decade. Not only monolayer TMDs but also their heterojunctions are considered useful for transistor applications [197-199]. These studies show a range of carrier mobilities, which is directly related to the quality of the TMDs.

The work intends to shed more light on the quality of our as-grown materials by electrical measurements. Especially our low temperature synthesized $\mathrm{MoS}_{2}$ samples, how its carrier mobility differs from high temperature synthesized samples. Finally, the influence of electron doping (tuning the Fermi-level) on the vibrational modes of $\mathrm{MoS}_{2} / \mathrm{WS}_{2}$ heterostructure device. 


\section{2}

\section{Field Effect Transistor}

Field effect transistor (FET) is a three terminals electronic device, widely used in digital integrated circuits. The three terminals are metallic electrodes i.e source, drain, and gate. FET uses a gate electrode potential $\mathrm{V}_{G}$ to control the conductivity of the semiconductor between the source and drain electrodes. The conduction path between drain and source is called a channel. In a FET, gate is practically separated from the entire body by a dielectric layer e.g $\mathrm{SiO}_{2}$. In particular, the field effect transistor (FET) works through the effect of an electric field in its active area. The dielectric of the device, which provides a medium for achieving electrostatic control of the channel by the gate, plays a very important role in influencing the performance of the device. Figure 6.1 shows a general structure of a field effect transistor.

\subsection{1}

\section{Operating Principle of FETs}

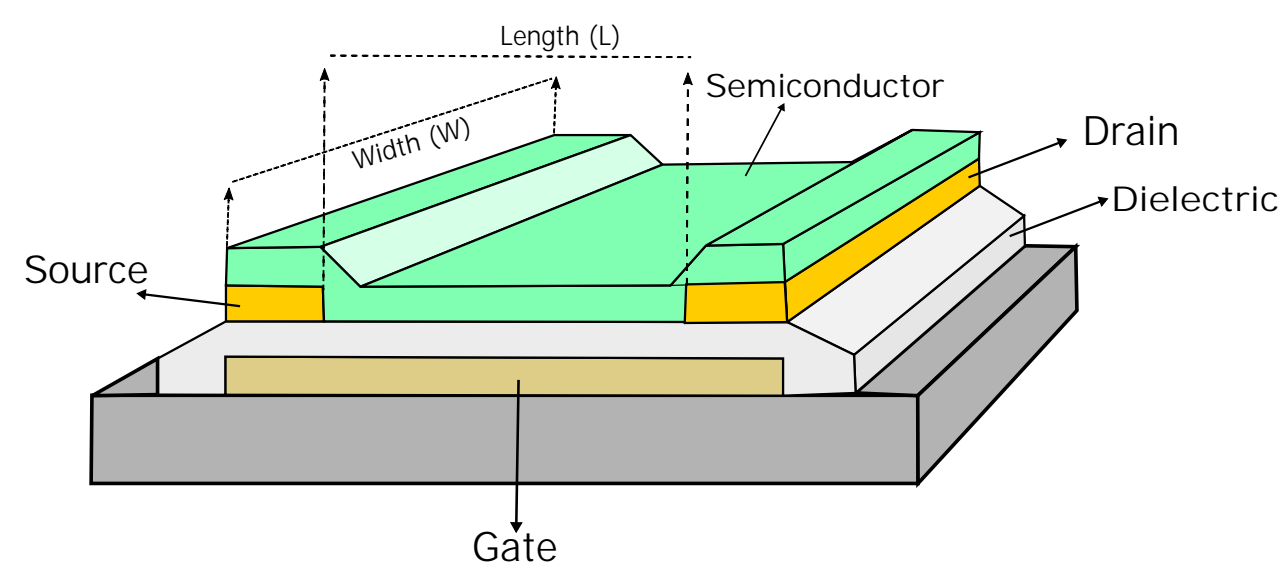

Figure 6.1: Illustration of the structure of a FET

The channel is the main part of a FET device, consisting of a thin semiconductor film sandwiched by two electrodes, the source (S) and the drain (D). The distance between the source and the drain is called length (L) of the channel. Width (W) of the channel is the area of a semiconductor touching the electrodes. A third electrode, called a gate electrode $(G)$, is located along the channel, between the source and the drain. This electrode is isolated from the others and the semiconductor film by a thin dielectric film, see figure 6.1. Therefore, FETs are governed by two perpendicular and independent electric fields i.e potential difference generated by the gate electrode and the source electrode $\mathrm{V}_{G}$, and the potential difference between the drain electrode and 
the source electrode $\mathrm{V}_{D S}$. This fact gives these devices a two-dimensional character.

A FET device is operated by controlling the $\mathrm{V}_{D S}$ potential and the $\mathrm{V}_{G}$ gate potential. Both $\mathrm{V}_{D S}$ and $\mathrm{V}_{G}$ simultaneously control the current between the drain and source $\mathrm{I}_{D S}$. When a positive $\mathrm{V}_{G}$ is applied, electrons within the semiconducting channel are attracted by the gate, forming an inversion layer that enables charge carrier transport. When the applied gate voltage is larger than the threshold voltage $V_{t h}$, the inversion layer connects the source and the drain, allowing conduction through the channel and thus switching on the transistor.

\section{3}

\section{Device Structure}

The structure of the devices considered in this work has a 1-3 nm thick TMDs (monolayer or bilayer). The devices were fabricated on p-doped $\mathrm{SiO}_{2} / \mathrm{Si}$ substrate by lithography process as explained in chapter 3 see section 3.9. The p-doped silicon substrate serves as the global back gate and the $\mathrm{SiO}_{2}$ as the dielectric [200]. The length of the channel ranges from $10-25 \mu \mathrm{m}$. The metallic electrodes source-drain thickness is taken as $50-80 \mathrm{~nm}$ and $\mathrm{Cu} / \mathrm{Au}$ is used as the source materials. The schematic representation of our device is shown in figure 6.2(a). Figure 6.2(b) shows an optical image of a back gate FET device fabricated on an $\mathrm{MoS}_{2}$ triangle.

(a)

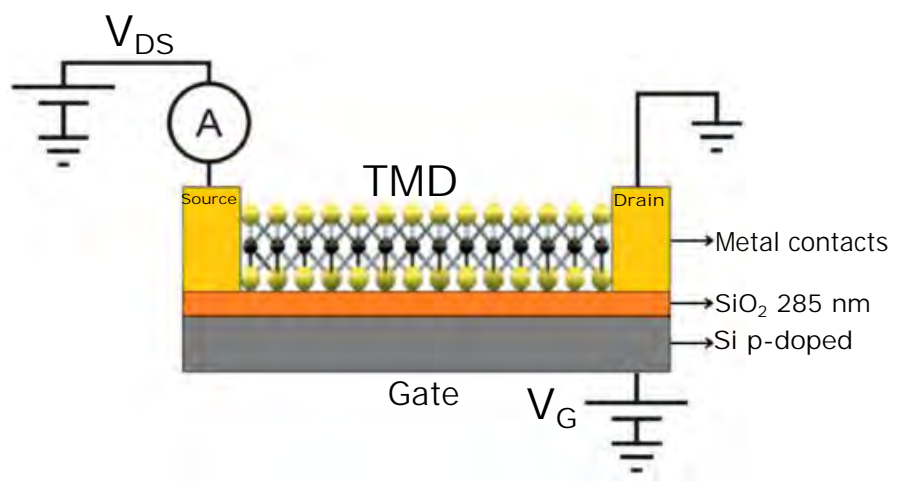

(b)

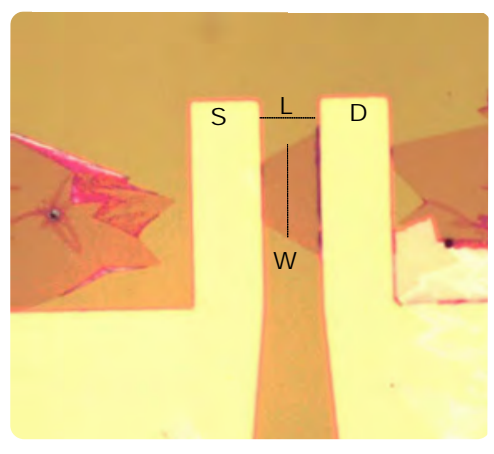

Figure 6.2: (a) Schematic representation of FET devices. (b) Optical image of a back gate FET device fabricated on $\mathrm{SiO}_{2} / \mathrm{Si}$, showing drain and source electrodes and channels L and W. 
We have calculated the channel conductivity $\sigma$ using the formula:

$$
\sigma=\frac{L}{W} \frac{I_{D S}}{V_{D S}}
$$

Here L and W are the length and width of the channel. Sheet conductivity is a normalized quantity which allows to compare samples and to extract quantities like field-effect mobility. This quantity does not take into account contact resistance but provides qualitative information on device behaviour. The electron mobility of the device is obtained by using the standard formula:

$$
\mu=\frac{1}{C_{o} \cdot V_{D S}} \frac{L}{W} \frac{\Delta I_{D S}}{\Delta V_{G}}
$$

The derivative in equation (6-2) is the maximum slope in the transfer characteristics. Where $\mathrm{C}_{o}$ is the gate capacitance and its value is

$$
C_{o}=\frac{\epsilon_{o} \kappa_{r}}{d}
$$

where

$\epsilon_{o}$ is permitivity of free space $=8.854 \times 10^{-12} \mathrm{~m}^{-3} \mathrm{~kg}^{-1} \mathrm{~s}^{4} \mathrm{~A}^{2}$.

$\kappa_{r}$ is the dielectric constant $=3.9$ for $\mathrm{SiO}_{2}$.

d is the thickness of $\mathrm{SiO}_{2}$ layer i.e $285 \mathrm{~nm}$.

Evaluating eq 6-3

$$
C_{o}=\frac{8.854 \times 10^{-12} \times 3.9}{285 \times 10^{-9}} \mathrm{Fcm}^{-2}
$$

Therefore $\mathrm{C}_{o}=1.21 \times 10^{-8} \mathrm{Fcm}^{-2}$ for $285 \mathrm{~nm} \mathrm{SiO}{ }_{2} \cdot \mathrm{V}_{D S}$ and $\mathrm{I}_{D S}$ are the drain-source voltage and current. $V_{G}$ is the back gate voltage.

\section{4}

\section{Transport Measurement of $\mathrm{MoS}_{2}$}

We investigated the electrical properties of our low temperature synthesized $\mathrm{MoS}_{2}$ crystals by fabricating two probe device on the as-grown sample. We applied the drain-source voltage $\left(\mathrm{V}_{D S}\right)$ from the electrodes. The back-gate voltage from the p-doped $\mathrm{Si}$ substrate, where the $285 \mathrm{~nm} \mathrm{SiO}_{2}$ serves as a back gate dielectric. Figure 6.3(a) presents the transfer characteristic curve of a FET device at room temperature, showing the typical n-type behaviour. Figure 6.3(b) shows the drain-source current $\left(\mathrm{I}_{D S}\right)$ as a function of source-drain voltage $\left(\mathrm{V}_{D S}\right)$ at different gate voltages. 
(a) $\mathrm{MoS}_{2}$ single crystal

(b)

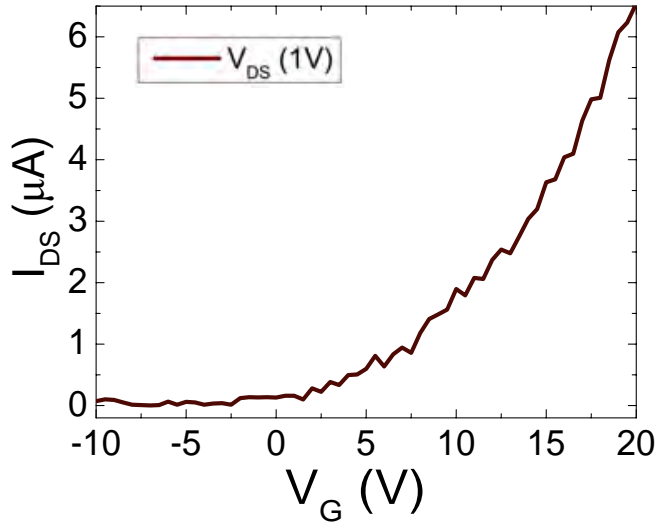

(c) $\mathrm{MoS}_{2}$ polycrystal

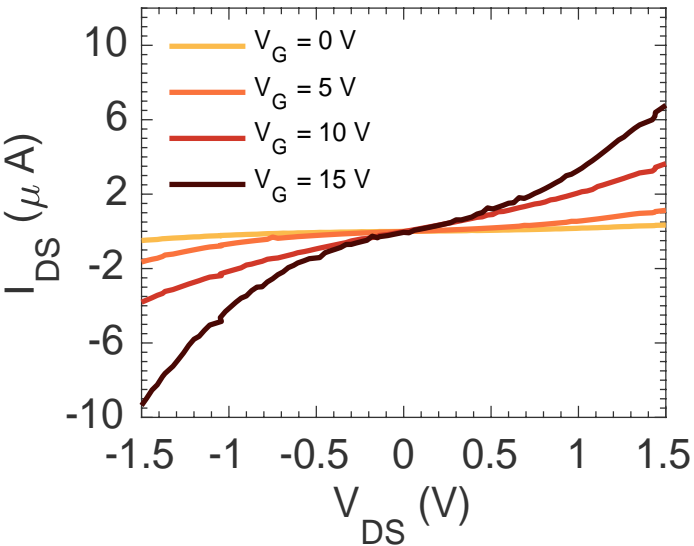

(d)
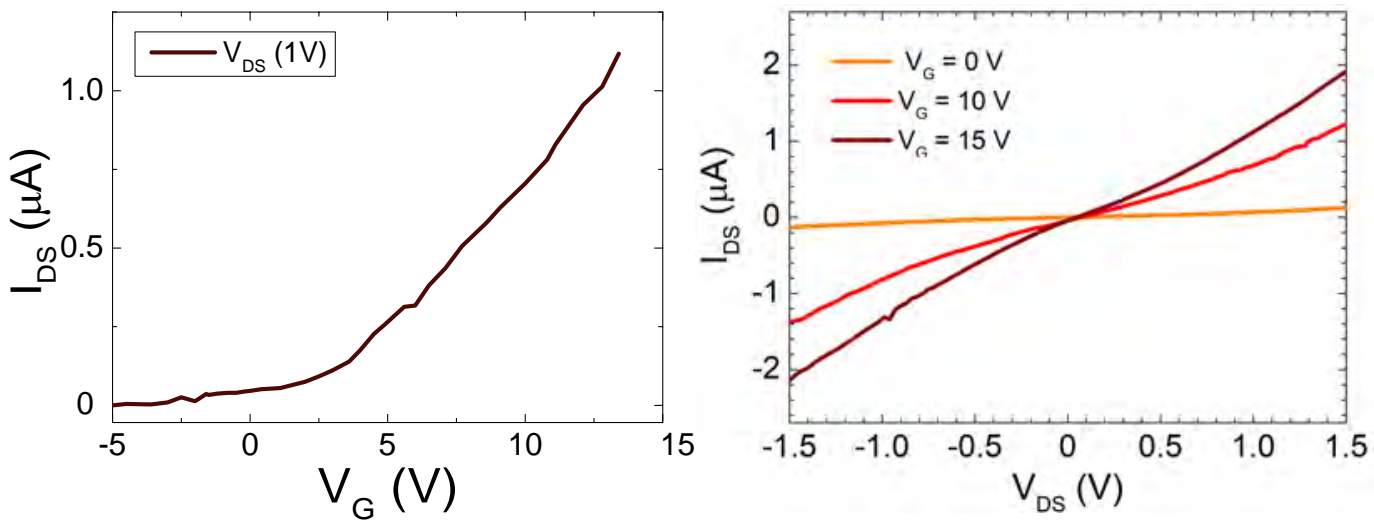

Figure 6.3: Transport measurements. (a) Transfer curve $\left(\mathrm{I}_{D S}\right.$ vs. $\mathrm{V}_{G}$ ) with $\mathrm{V}_{D S}$ fixed at $1 \mathrm{~V}$, for $\mathrm{MoS}_{2}$ single crystal. (b) Output curve $\left(\mathrm{I}_{D S}\right.$ vs. $\mathrm{V}_{D S}$ ) at different $\mathrm{V}_{G}$ values, for single crystal. (c) Transfer curve ( $\mathrm{I}_{D S}$ vs. $\left.\mathrm{V}_{G}\right)$ with $\mathrm{V}_{D S}$ fixed at $1 \mathrm{~V}$ for $\mathrm{MoS}_{2}$ polycrystalline film. (d) Output curve $\left(\mathrm{I}_{D S}\right.$ vs. $\left.\mathrm{V}_{D S}\right)$ at different $\mathrm{V}_{G}$ values, for polycrystalline film.

Our single crystal $\mathrm{MoS}_{2}$ device shows high electrical performance and the average electrical conductivity is estimated from the $\left(\mathrm{I}_{D S}\right)$ vs $\left(\mathrm{V}_{D S}\right)$ curve at $0\left(\mathrm{~V}_{G}\right), \sim 8.9 \times 10^{-7} \Omega^{-1}$. The mobility is estimated as $9.8 \pm 0.7 \mathrm{~cm}^{2} V^{-1} \mathrm{~s}^{-1}$ which is comparable with the previous reports [28,32, 69, 181, 201].

Similarly, the transfer characteristic curve and drain-source current $\left(\mathrm{I}_{D S}\right)$ vs. source-drain voltage $\left(\mathrm{V}_{D S}\right)$ for the polycrystalline film can be found at figure $6.3(\mathrm{c}, \mathrm{d})$. Transfer curve for polycrystalline film is linear and symmetric compared to single crystal curve. The conductivity for polycrystalline film is almost equal to single crystal, $\sim 8 \times 10^{-7} \Omega^{-1}$. We estimated the mobility for the polycrystalline film at $2.7 \pm 0.5 \mathrm{~cm}^{2} V^{-1} \mathrm{~s}^{-1}$. The relatively low electron mobility of the film is likely due to the presence of domain boundaries in the polycrystals, which could trap electrons and affect the charge transfer. Typically, in high-temperature synthesis, it is common to observe gate voltage 
leakage through pinholes caused by the destruction of a thin $\mathrm{SiO}_{2}$ layer. Another advantage of low-temperature synthesis is the possibility to direct fabrication of the device, avoiding the transfer procedure.

\section{5}

\section{Transport Measurement of $\mathrm{WS}_{2}$}

(a)

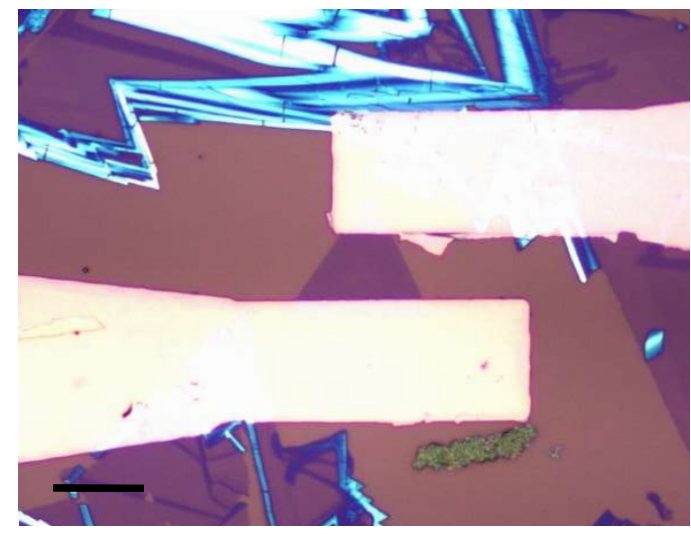

(b)

(c)
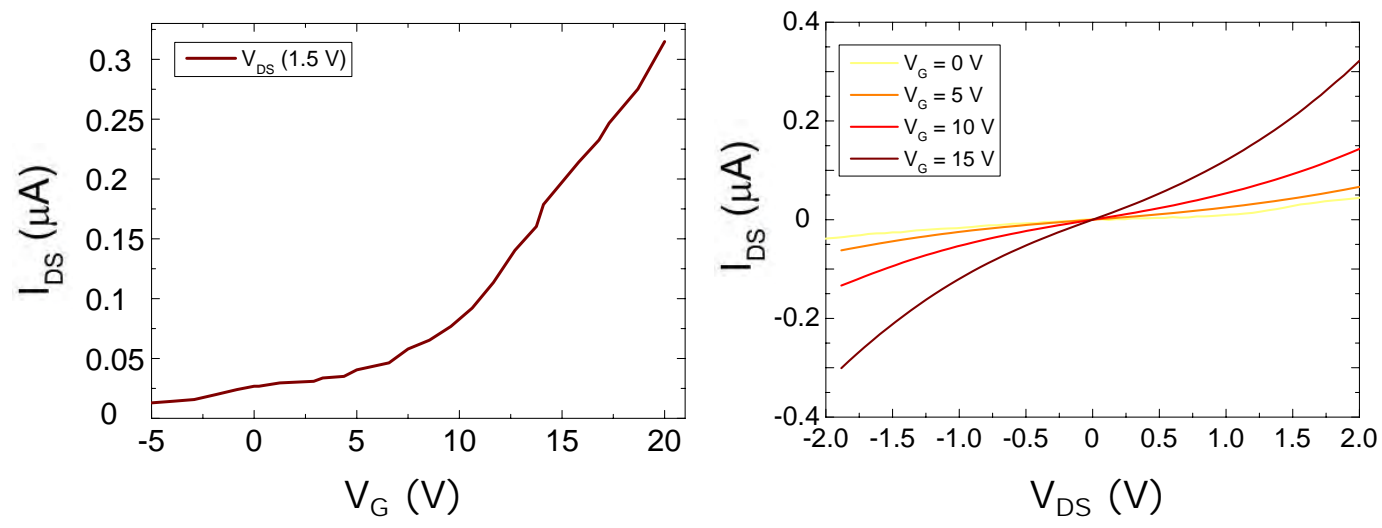

Figure 6.4: (a) Optical image of a FET device fabricated after transferring $\mathrm{WS}_{2}$ on $\mathrm{SiO}_{2} / \mathrm{Si}$ substrate, scale bar is $10 \mu \mathrm{m}$. (b) Transfer curve $\left(\mathrm{I}_{D S}\right.$ vs. $\left.\mathrm{V}_{G}\right)$ with $\mathrm{V}_{D S}$ fixed at $1.5 \mathrm{~V}$ for $\mathrm{WS}_{2}$. (c) Output curve of $\mathrm{WS}_{2}\left(\mathrm{I}_{D S}\right.$ vs. $\left.\mathrm{V}_{D S}\right)$ for different $\mathrm{V}_{G}$ values.

Figure 6.4(a) depicts the optical image of the $\mathrm{WS}_{2}$ FET device. $\mathrm{WS}_{2}$ device was fabricated on the samples transferred on the $\mathrm{SiO}_{2} / \mathrm{Si}$ substrate after growth to avoid any kind of leakage current from the $\mathrm{SiO}_{2}$ dielectric. The transfer characteristic curve for $\mathrm{WS}_{2}$ device is shown in figure 6.4(b). The output curve $\left(\mathrm{I}_{D S}\right.$ vs. $\left.\mathrm{V}_{D S}\right)$ of $\mathrm{WS}_{2}$ was also measured see figure 6.4(c). $\mathrm{WS}_{2}$ also shows n-type behaviour, the conductivity and mobility was also calculated for $\mathrm{WS}_{2}$. The conductivity and mobility for $\mathrm{WS}_{2}$ is $\sim 7 \times 10^{-9}$ 
$\Omega^{-1}$ and $\sim 0.53 \pm 0.1 \mathrm{~cm}^{2} V^{-1} s^{-1}$ which is much lower compared to $\mathrm{MoS}_{2}$. Relatively high mobility of $\mathrm{MoS}_{2}$ compared to $\mathrm{WS}_{2}$ shows sodium mediated low temperature synthesis grows better samples compared to high temperature synthesized $\mathrm{WS}_{2}$.

\section{6}

\section{Transport Measurement of $\mathrm{MoS}_{2} / \mathrm{WS}_{2}$ Heterostructure}

Figure 6.5(a) shows the optical image of $\mathrm{MoS}_{2} / \mathrm{WS}_{2}$ heterostructure FET device on $\mathrm{SiO}_{2} / \mathrm{Si} 285 \mathrm{~nm}$ substrate. The bottom triangle is the $\mathrm{MoS}_{2}$ and on top of it is an incomplete grown $\mathrm{WS}_{2}$ triangle. From the optical image, it seems a $0^{\circ}(3 \mathrm{R})$ stacked heterostructure. The drain-source current $\left(\mathrm{I}_{D S}\right)$ as a function of source-drain voltage $\left(\mathrm{V}_{D S}\right)$ at different gate voltages is shown in Figure 6.5(b). The conductivity estimated for $\mathrm{MoS}_{2} / \mathrm{WS}_{2}$ heterostructure, $\sim 2 \times 10^{-6} \Omega^{-1}$ which is highest compared to the other samples. Generally, CVD grown $\mathrm{MoS}_{2} / \mathrm{WS}_{2}$ vertical heterostructure are know to have high mobility than its counterparts [202]. Table 6.1 shows the comparison of mobility and conductivity of all of our devices.

(a)

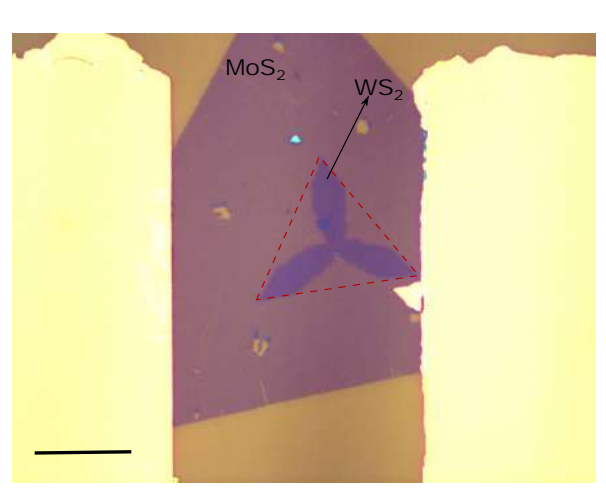

(b)

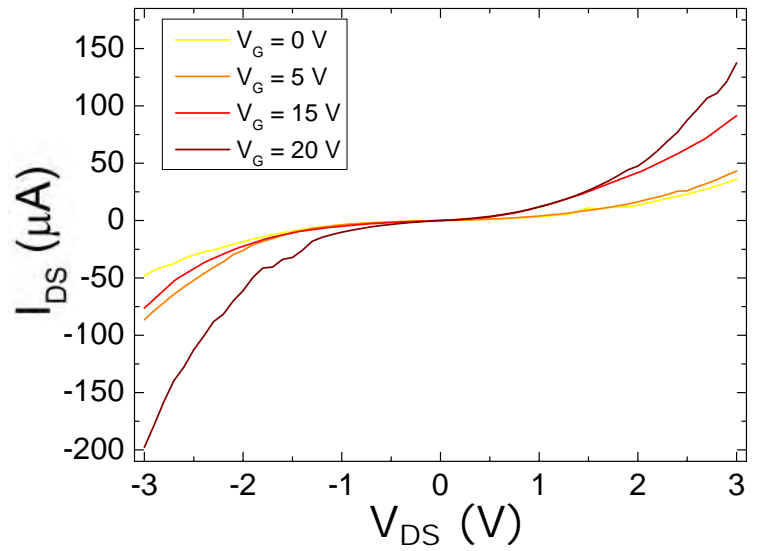

Figure 6.5: (a) Optical image of $\mathrm{MoS}_{2} / \mathrm{WS}_{2}$ heterostructure device on $\mathrm{SiO}_{2} / \mathrm{Si}$ $285 \mathrm{~nm}$ substrate. The big triangle is the $\mathrm{MoS}_{2}$ and on top of it flower shape is an incomplete grown $\mathrm{WS}_{2}$ triangle. (b) Output curve of $\mathrm{MoS}_{2} / \mathrm{WS}_{2}\left(\mathrm{I}_{D S}\right.$ vs. $\mathrm{V}_{D S}$ ) for different $\mathrm{V}_{G}$ values.

Strictly speaking, this is not the precise way of extracting conductivity and mobility. The exact value of conductivity needs four probe measurements rather than two probe, where we have minimum contact resistance and the shape of the TMD sheet should be rectangular. The standard complementary metal-oxide semiconductor technology requires equal and small values of 
$\mathrm{V}_{D S}$ and $\mathrm{V}_{G}$ applied to determine these quantities. Our results are rather a comparison between our grown samples quality.

\begin{tabular}{|l||c|c|}
\hline Sample & $\begin{array}{c}\text { Average Conductivity } \\
\text { at } 0\left(\mathrm{~V}_{G}\right) \Omega^{-1}\end{array}$ & $\begin{array}{c}\text { Mobility } \\
\mathrm{cm}^{2} V^{-1} \mathrm{~s}^{-1}\end{array}$ \\
\hline \hline $\mathrm{MoS}_{2}$ single crystal & $8.9 \times 10^{-7}$ & $9.8 \pm 0.7$ \\
\hline $\mathrm{MoS}_{2}$ polcrystals & $8 \times 10^{-7}$ & $2.7 \pm 0.5$ \\
\hline $\mathrm{WS}_{2}$ & $7 \times 10^{-9}$ & $0.53 \pm 0.1$ \\
\hline $\mathrm{MoS}_{2} / \mathrm{WS}_{2}$ & $2 \times 10^{-6}$ & - \\
\hline
\end{tabular}

Table 6.1: Average conductivity and mobility of TMDs.

\section{7}

\section{Defect Related Raman Peak Reveled by Electo-Raman of $\mathrm{MoS}_{2} / \mathrm{WS}_{2}$} Device

Consider a voltage is applied across our device which will produce electric field. This electric field accumulates free or fixed charges at the interface between the oxide and the semiconductor throughout our TMD device. Such charges can effectively dope the semiconductor TMD in the region near the interface, and thus shifting the Fermi level. This shift in Fermi level could result in shifting of phonon modes. This effect is commonly used in electronic and optoelectronic devices to tune the conductivity as well as the electrical barrier in the devices. The gate voltage $\left(\mathrm{V}_{G}\right)$ creates an electrostatic potential difference between the sample and the gate electrode, and the addition of charge carriers leads to a shift in the Fermi level.

We measured the Raman spectra of $\mathrm{MoS}_{2} / \mathrm{WS}_{2}$ device shown in figure 6.5(a) and simultaneously applied the gate voltage. Figure 6.6 plots the Raman spectra at different values of back-gate voltage with $473 \mathrm{~nm}$ laser. After every step i.e increase in gate voltage, Raman spectrum was acquired after stabilization of the current $\mathrm{I}_{D S}$. Each spectrum is collected at room temperature under ambient pressure for $50 \mathrm{sec}$. Figure 3.19 shows a schematic of the experimental setup of the Raman spectroscopy measurements with voltage.

Four prominent peaks were observed belonging to the in-plane and out of plane vibrational modes of $\mathrm{WS}_{2}$ and $\mathrm{MoS}_{2}$ layers. However, Raman signal from $\mathrm{WS}_{2}$ (top layer) is stronger as compared to $\mathrm{MoS}_{2}$. All the Raman peaks in figure 6.6 are fitted with Lorentzian function. Red dots are the experimental data, the black lines are fitted Lorentzians. 


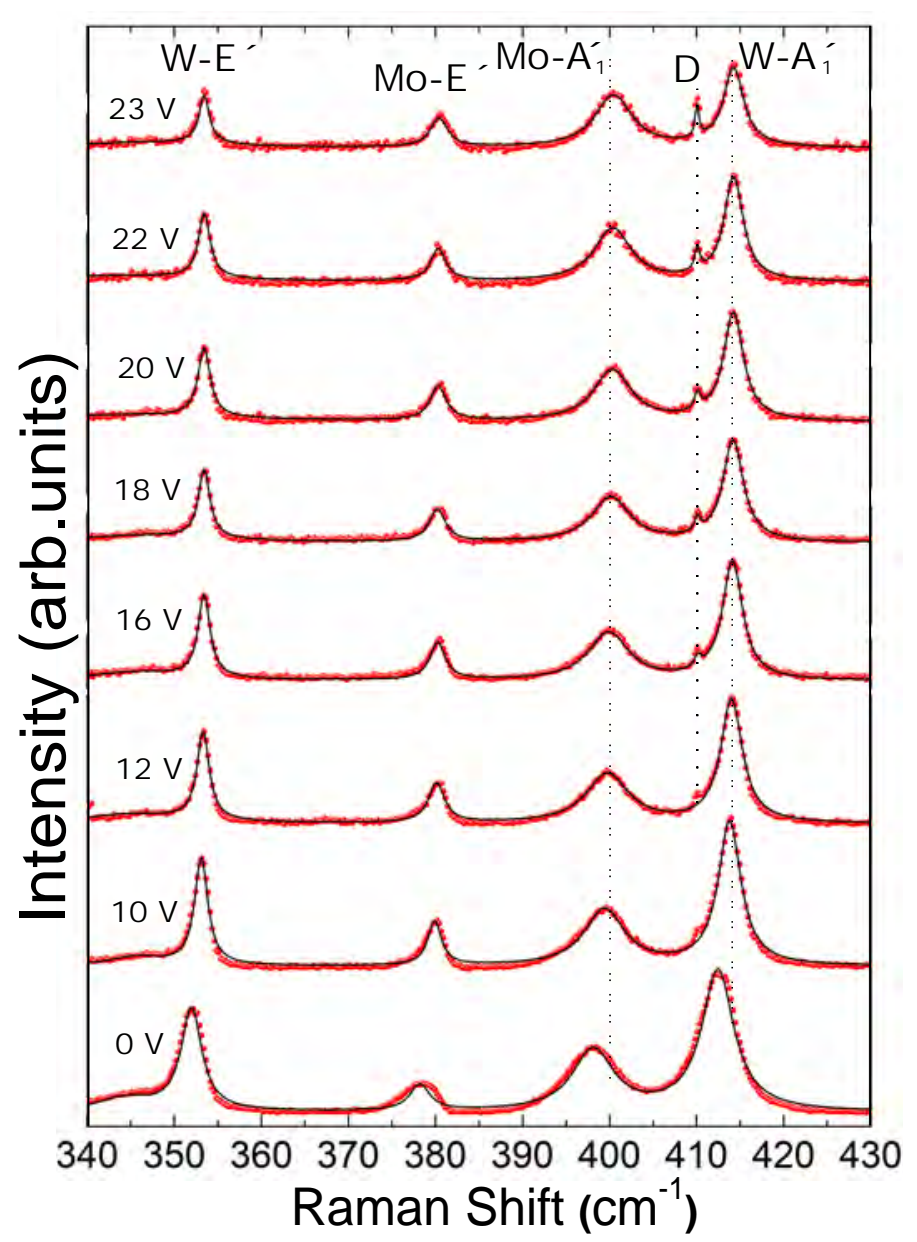

Figure 6.6: Raman spectra of $\mathrm{MoS}_{2} / \mathrm{WS}_{2}$ device at different back gate voltages $\mathrm{V}_{G}$, laser wavelength $473 \mathrm{~nm}$. Red circles are experimental data points, the black lines are Lorentzian fits to the total spectrum.

Figure 6.7(a) represents the dependence of peak position with applied gate voltage. The $\mathrm{A}_{1}^{\prime}$ modes show strong doping dependence, compared to $\mathrm{E}^{\prime}$. The $\mathrm{W}$-A $\mathrm{A}_{1}^{\prime}$ mode shows blueshift of $\sim 4 \mathrm{~cm}^{-1}$, while Mo- $\mathrm{A}_{1}^{\prime}$ also shows blueshift of $\sim 3 \mathrm{~cm}^{-1}$ with gate voltage. The FWHM of W-A $\mathrm{A}_{1}^{\prime}$ and Mo- $\mathrm{A}_{1}^{\prime}$ extracted from lorenzian fit, decreases in both the cases see figure 6.7(b). This blueshift and decrease in FWHM is a result of electron doping. It is previously reported that the G-Band of graphene blueshifts and FWHM decreases with the electron and hole doping $[193,196,203]$. The FWHM of the Lorentzian components gives relevant information on the phonon lifetime. The intensity of the $\mathrm{W}-\mathrm{A}_{1}^{\prime}$ and Mo- $\mathrm{A}_{1}^{\prime}$ mode is also attenuated by the high carrier density see figure 6.8(a).

A new peak emerges after $\mathrm{V}_{G}=10 \mathrm{~V}$, at $\sim 410 \mathrm{~cm}^{-1}$ which is related to defects (D) from the $\mathrm{WS}_{2}$ crystal. Defects in the sample can be introduced during the (CVD) growth, device fabrication, or by electron doping. These defects are expected to worsen the device quality. However, these defects can also be beneficial for our device functionalities. Defects have been shown to 
(a)

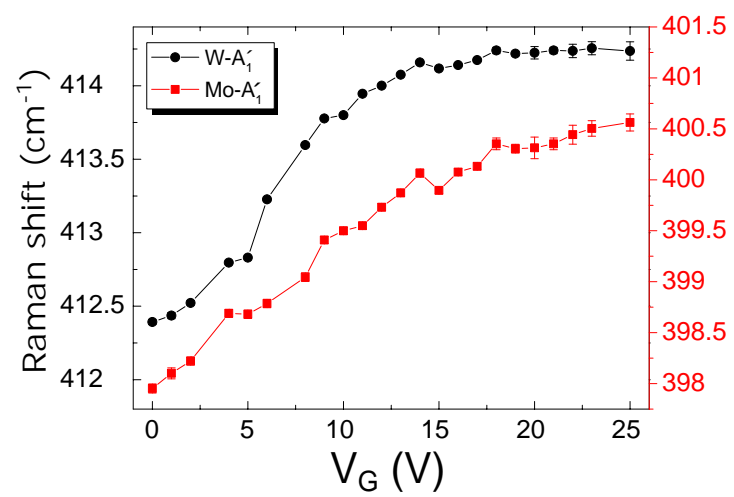

(b)

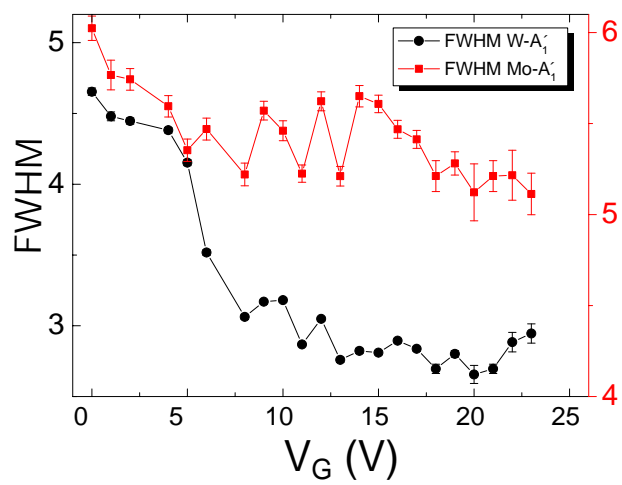

Figure 6.7: (a) Peak position of $\mathrm{W}-\mathrm{A}_{1}^{\prime}$ and Mo- $\mathrm{A}_{1}^{\prime}$ as a function of $\mathrm{V}_{G}$. (a) FWHM of W-A $\mathrm{A}_{1}^{\prime}$ and Mo- $\mathrm{A}_{1}^{\prime}$ peak as a function of $\mathrm{V}_{G}$.

(a)

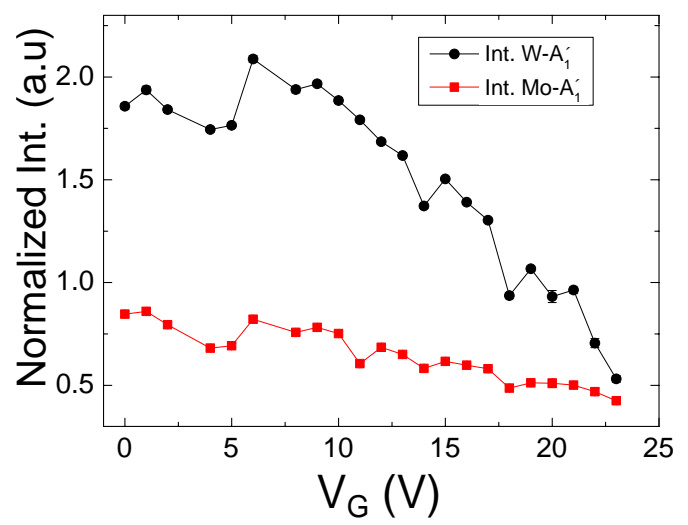

(b)

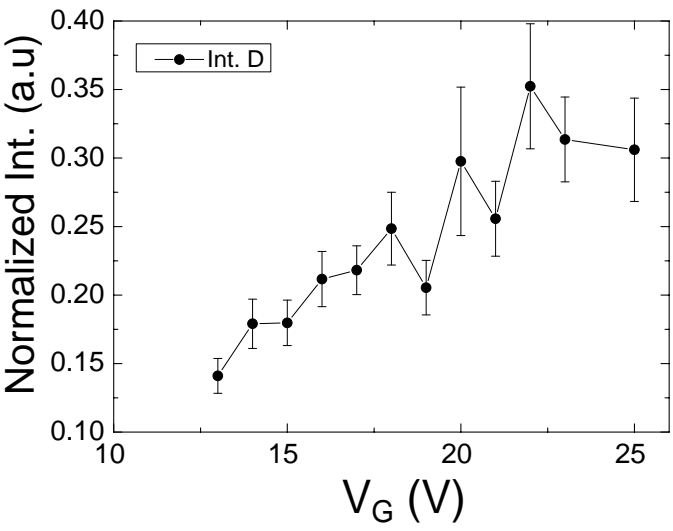

Figure 6.8: (a) Intensity normalized with $\mathrm{Si}$ peak Mo- $\mathrm{A}_{1}^{\prime}$ and $\mathrm{W}-\mathrm{A}_{1}^{\prime}$ as a function of $\mathrm{V}_{G}$. (b) Normalized intensity of $\mathrm{D}$ peak as a function of $\mathrm{V}_{G}$.

be the dominant dopant in $\mathrm{MoS}_{2}$, and allows n-type and p-type regions to coexist across the regions of the same sample [204]. This D peak is most likely present in the Raman spectrum as shoulder peak with W-A $\mathrm{A}_{1}^{\prime}$. Blueshifting of $\mathrm{W}-\mathrm{A}_{1}^{\prime}$ peak have made it prominent. There is a strong correlation between the intensity of the $\mathrm{D}$ and $\mathrm{A}_{1}^{\prime}$ peaks. The intensity of the $\mathrm{D}$ peak becomes more prominent with increase of gate voltage see figure $6.8(\mathrm{~b})$, while the intensity of $\mathrm{A}_{1}^{\prime}$ peaks shows opposite behavior see figure 6.8(a). The prominent appearance the $\mathrm{D}$ peak shows the density of defects. There is not much known about this $\mathrm{D}$ peak in the Raman spectrum. This peak was first reported by Chanwoo Lee et. al [205] by Tip-enhance Raman spectroscopy of monolayer $\mathrm{WS}_{2}$. The observed defect-related Raman mode can be utilized to evaluate the quality of our 
sample and will be helpful to improve the performance of future optoelectronic devices.

We also did the electro-Raman measurements on $\mathrm{WS}_{2}$ monolayer device. But no noticeable shift in the peaks were observed, likely due to the low conductivity of $\mathrm{WS}_{2}$ device. More studies are required on the electro-Raman measurements to observe doping effects in different TMDs. The top gate FET devices might be a better option for higher doping at low gate voltage.

\section{8}

\section{Partial Conclusion}

In summary, we have investigated the transport properties of monolayer $\mathrm{MoS}_{2}, \mathrm{WS}_{2}$, and their heterostructure. The devices were fabricated by photolithography on the samples grown in the same CVD system, and characterized under the same ambient conditions to investigate their comparative performance. The back-gated, $\mathrm{MoS}_{2} / \mathrm{WS}_{2}$ heterostructure device show higher performance compared to the others. On the other hand, low temperature synthesised $\mathrm{MoS}_{2}$ shows higher carrier mobility compared to $\mathrm{WS}_{2}$. Further, we have demonstrated the electron doping dependence of Raman modes of $\mathrm{MoS}_{2} / \mathrm{WS}_{2}$ device. The $\mathrm{A}_{1}^{\prime}$ peaks show blueshift following the decrease in FWHM, as result of change in Fermi level. A new peak emerges after sufficient electron doping following $\mathrm{W}-\mathrm{A}_{1}^{\prime}$ blueshift. This peak (D) is attributed to the defects in $\mathrm{WS}_{2}$ layer. 


\section{7}

\section{Conclusions and Future Work}

In this thesis, we have reported the atmospheric pressure chemical vapor deposition (APCVD) synthesis of monolayer and bilayers $\mathrm{MoS}_{2}, \mathrm{WS}_{2}$, and their vertical heterostructure. We pointed out key elements in the CVD process for repeatable synthesis recipes. A new synthesis route was established mediated by sodium which leads us to the successful growth of monolayer $\mathrm{MoS}_{2}$ single crystal and polycrystalline film at low temperature $\left(550^{\circ} \mathrm{C}\right)$. The DFT calculation also shows that the critical element is the number of sodium in the system, which reduces the energy formation and allows to use a reduced temperature $\left(\Delta T=250^{\circ} \mathrm{C}\right)$. Single crystals were grown by controlling the $\mathrm{Mo} / \mathrm{Na}$ ratio and polycrystals by increasing the growth time. We characterized the grown monolayers by optical microscopy, X-ray photoelectron spectroscopy (XPS), photoluminescence, Raman spectroscopy, atomic force microscopy (AFM), and transport experiments. The measurements confirm its monolayer thickness, crystallinity, optical and electrical properties. The analysis of Raman defective peak LA shows the same amount of defects in single and polycrystals. The optical and electrical performances are similar to those grown at high temperatures. This work would serve as a reference for the low-temperature synthesis of other TMDs.

The second harmonic generation (SHG) was studied on APCVD synthesised two and three layer samples. The SHG shows that the layers in bilayers grown with $0^{\circ}$ have no inversion symmetry refers to $3 \mathrm{R}(\mathrm{AB})$ phase stacking. For $60^{\circ}$, we have an inversion symmetry (Centrosymmetric) and $2 \mathrm{H}\left(\mathrm{AA}^{\prime}\right)$ phase stacking. These studies show that for CVD grown bilayer samples we can achieve both $2 \mathrm{H}\left(\mathrm{AA}^{\prime}\right)$ and $3 \mathrm{R}(\mathrm{AB})$ phases.

The transport properties of monolayer $\mathrm{MoS}_{2}$ and $\mathrm{WS}_{2}$ and heterostructure was studied by fabricating devices on them. The back-gated, CVD grown $\mathrm{MoS}_{2} / \mathrm{WS}_{2}$ based device show higher performance compared to the others. Our study demonstrates high quality of material and further roots towards device optimization. We demonstrated the electron doping dependence of Raman modes of $\mathrm{MoS}_{2} / \mathrm{WS}_{2}$ device. The $\mathrm{A}_{1 g}$ peaks show a blueshift following the decrease in FWHM, as a result of the change in Fermi level. A new peak emerges after sufficient electron doping. This peak $\left(\mathrm{D}^{\prime}\right)$ is attributed to the 
defects in $\mathrm{WS}_{2}$ layer.

Due to their uniqueness and diversity, TMDs are of far-reaching importance in scientific research and have various potential applications in future optoelectronic device technology. The direct bandgap and controllable valley polarization give TMDs promising opportunities in novel optoelectronics applications. Over 40 TMDs have been discovered yet [8], and the Van der Waals heterostructures gives infinite opportunities for new 2D-TMDs materials. We purpose the following works for the future on this topic:

A lot of work is already being done on $\mathrm{MoS}_{2}$ and $\mathrm{WS}_{2}$, we should move on to the synthesis of other TMDs e.g selenides and tellurides. Catalytic behaviour of sodium could be used in the synthesis of other TMDs. Since the theoretical calculations are fully in agreement with the experimental results, first principle calculations (DFT) before growing any new TMD, will be very helpful. More studies are needed on the synthesis of TMDs on glass. One should choose glass with low sodium concentration, which will help in growing clean samples and avoids multiple layers. Little is known about the transmission properties of CVD grown TMDs, direct synthesis on the ordinary glass will help in understanding the transmission properties and achieving novel flexible devices. Artificially stacked heterostructures, as well as CVD synthesized, opens an entirely new field of research, we should move on to the heterostructures of selenides, and sulphides which could provide us the controllable bandgap. We will try to synthesize new TMDs heterostructures and other 2D materials by CVD. The alternative way to achieve new heterostructures is transferring one TMD on another. We will search for the best candidates for fast-response, broadband photo-detecting, and high-efficiency light emission.

Second harmonic generation (SHG) should be studied on new artificially stacked layers e.g selenides and tellurides. $\mathrm{WSe}_{2}$ field-effect transistor has already proved to be a new class of electrically tunable nonlinear optical device $[58,123]$. We purpose to study the SHG in our heterostructure FET device. Also, the devices fabricated on different stacked heterostructures (i.e $0^{\circ}$ and $60^{\circ}$ ) could be interesting to study. It will be interesting to see either nonlinear electric susceptibilities is tunable through electrostatic doping in a bilayer/heterostructure FET. A more interesting feature will be to check either it is possible to break the inversion symmetry in $2 \mathrm{H}$ stacked bilayer through electrostatic doping. 
Improvements are needed in our back gate FET devices. Improved device engineering, including better gating schemes and waveguide integration, will enable enhanced tunability. We can reach much higher doping level with top gating than standard $\mathrm{SiO}_{2}$ back gating. A suitable polymer electrolyte having high capacitance should be chosen as top gating material. The polymer should be transparent, which allows us to perform the optical measurements with simultaneous electrical characterization. 


\section{Bibliography}

[1] GEIM, A. K.; NOVOSELOV, K. S.. The rise of graphene. Nat. Mater., 6(3):183-191, 2007.

[2] SCHWIERZ, F.. Graphene transistors. Nat. Nanotechnol., 5(7):487496, 2010.

[3] TIWARI, S. K.; SAHOO, S.; WANG, N. ; HUCZKO, A.. Graphene research and their outputs: Status and prospect. J. Sci. Adv. Mater. Devices, 5(1):10-29, 2020.

[4] BERNARDI, M.; ATACA, C.; PALUMMO, M. ; GROSSMAN, J. C.. Optical and Electronic Properties of Two-Dimensional Layered Materials. Nanophotonics, 6(2):479-493, 2017.

[5] SAMADI, M.; SARIKHANI, N.; ZIRAK, M.; ZHANG, H.; ZHANG, H. L. ; MOSHFEGH, A. Z.. Group 6 transition metal dichalcogenide nanomaterials: Synthesis, applications and future perspectives. Nanoscale Horizons, 3(2):90-204, 2018.

[6] CHHOWALLA, M.; LIU, Z. ; ZHANG, H.. Two-dimensional transition metal dichalcogenide (TMD) nanosheets. Chem. Soc. Rev., 44(9):2584-2586, 2015.

[7] DUAN, X.; WANG, C.; PAN, A.; YU, R. ; DUAN, X.. Two-dimensional transition metal dichalcogenides as atomically thin semiconductors: Opportunities and challenges. Chem. Soc. Rev., 44(24):88598876, 2015.

[8] MD NURUNNABI, J. M.. Biomedical Applications of Graphene and 2D Nanomaterials, 1st Edition, volumen 1. Elsevier, 2019.

[9] WILSON, J. A.; YOFFE, A. D.. The transition metal dichalcogenides discussion and interpretation of the observed optical, electrical and structural properties. Adv. Phys., 18(73):193-335, 1969.

[10] FRINDT, R. F.; YOFFE, A. D.. Physical Properties of Layer Structures: Optical Properties and Photoconductivity of Thin Crys- 
tals of Molybdenum Disulphide. Proc. R. Soc. A Math. Phys. Eng. Sci., 273(1352):69-83, 1963.

[11] SPLENDIANI, A.; SUN, L.; ZHANG, Y.; LI, T.; KIM, J.; CHIM, C. Y.; GALLI, G. ; WANG, F.. Emerging photoluminescence in monolayer MoS2. Nano Lett., 10(4):1271-1275, 2010.

[12] LI, H.; WU, J.; YIN, Z. ; ZHANG, H.. Preparation and applications of mechanically exfoliated single-layer and multilayer $\mathrm{MoS}_{2}$ and WSe $_{2}$ nanosheets. Acc. Chem. Res., 47(4):1067-1075, 2014.

[13] YUAN, L.; GE, J.; PENG, X.; ZHANG, Q.; WU, Z.; JIAN, Y.; XIONG, X.; YIN, H. ; HAN, J.. A reliable way of mechanical exfoliation of large scale two dimensional materials with high quality. AIP Adv., 6(12), 2016.

[14] AMBROSI, A.; PUMERA, M.. The CVD graphene transfer procedure introduces metallic impurities which alter the graphene electrochemical properties. Nanoscale, 6(1):472-476, 2014.

[15] LIN, Z.; THEE, M. T.; ELÍAS, A. L.; FENG, S.; ZHOU, C.; FUJISAWA, K.; PEREA-LÓPEZ, N.; CAROZO, V.; TERRONES, H. ; TERRONES, M.. Facile synthesis of $\mathrm{MoS}_{2}$ and $\mathrm{Mo}_{x} \mathrm{~W}_{1-x} \mathrm{~S}_{2}$ triangular monolayers. APL Mater., 2(9):092514, 2014.

[16] WANG, X.; GONG, Y.; GANG, S.; CHOW, W. L.; KEYSHAR, W.; YE, G.; VAJTAI, R.; LOU, J.; LIU, Z.; RINGE, E.; TAY, B. K. ; AJAYAN, P. M.. Chemical vapor deposition growth of Crystalline Monolayer MoSe2. ACS Nano, 8(5):5125-5131, 2014.

[17] LEE, Y. H.; ZHANG, X. Q.; ZHANG, W.; CHANG, M. T.; LIN, C. T.; CHANG, K. D.; YU, Y. C.; WANG, J. T.; CHANG, C. S.; LI, L. J. ; LIN, T. W.. Synthesis of large-area $\mathbf{M o S}_{2}$ atomic layers with chemical vapor deposition. Adv Mater, 24(17):2320-2325, 2012.

[18] ZHAO, W. F.; YU, H.; LIAO, M. Z.; ZHANG, L.; ZOU, S. Z.; YU, H. J.; HE, C. J.; ZHANG, J. Y.; ZHANG, G. Y. ; LIN, X. C.. Large area growth of monolayer $\mathrm{MoS}_{2}$ film on quartz and its use as a saturable absorber in laser mode-locking. Semicond. Sci. Technol., 32(2), 2017.

[19] HAMMETT, B.; RESEARCH, BREANNE HAMMETT AND, A. . ; NANAYAKKARA, S.. Optimizing growth conditions for $2 \mathrm{D} \mathbf{M o S}_{2}$. ., 2017. 
[20] BILGIN, I.; LIU, F.; VARGAS, A.; WINCHESTER, A.; MAN, M. K.; UPMANYU, M.; DANI, K. M.; GUPTA, G.; TALAPATRA, S.; MOHITE, A. D. ; KAR, S.. Chemical Vapor Deposition Synthesized Atomically Thin Molybdenum Disulfide with Optoelectronic-Grade Crystalline Quality. ACS Nano, 9(9):8822-8832, 2015.

[21] MUKULIKA DINARA, C. S. R.. Two Dimensional Transition Metal Dichalcogenides, volumen 1. Springer Singapore, 2019.

[22] CHANG SEOK, L.; COSTEL SORIN, C.; WALEED, M.; BÉRENGÈRE, L.; MARC, C.; MARC, C.; FRANÇOIS LE, N. ; JEAN-LUC, M.. Synthesis of conducting transparent few-layer graphene directly on glass at $450^{\circ}$ C. Nanotechnology, 23(26):265603, 2012.

[23] CHEN, Y. Z.; MEDINA, H.; TSAI, H. W.; WANG, Y. C.; YEN, Y. T.; MANIKANDAN, A. ; CHUEH, Y. L.. Low temperature growth of graphene on glass by carbon-enclosed chemical vapor deposition process and its application as transparent electrode. Chem. Mater., 27(5):1646-1655, 2015.

[24] SUN, J.; CHEN, Y.; CAI, X.; MA, B.; CHEN, Z.; PRIYDARSHI, M. K.; CHEN, K.; GAO, T.; SONG, X.; JI, Q.; GUO, X.; ZOU, D.; ZHANG, Y. ; LIU, Z.. Direct low-temperature synthesis of graphene on various glasses by plasma-enhanced chemical vapor deposition for versatile, cost-effective electrodes. Nano Res., 8(11):3496-3504, 2015.

[25] PARK, B. J.; CHOI, J. S.; EOM, J. H.; HA, H.; KIM, H. Y.; LEE, S.; SHIN, H. ; YOON, S. G.. Defect-Free Graphene Synthesized Directly at $150{ }^{\circ} \mathrm{c}$ via Chemical Vapor Deposition with No Transfer. ACS Nano, 12(2):2008-2016, 2018.

[26] JANG, J.; SON, M.; CHUNG, S.; KIM, K.; CHO, C.; LEE, B. H. ; HAM, M. H.. Low-temperature-grown continuous graphene films from benzene by chemical vapor deposition at ambient pressure. Sci. Rep., 5(November):1-7, 2015.

[27] MARCHENA, M.; JANNER, D.; CHEN, T. L.; FINAZZI, V. ; PRUNERI, $\mathrm{V}$.. Low temperature direct growth of graphene patterns on flexible glass substrates catalysed by a sacrificial ultrathin $\mathrm{Ni}$ film. Opt. Mater. Express, 6(8):2487, 2016. 
[28] GONG, Y.; LIN, Z.; YE, G.; SHI, G.; FENG, S.; LEI, Y.; ELÍAS, A. L.; PEREA-LOPEZ, N.; VAJTAI, R.; TERRONES, H.; LIU, Z.; TERRONES, M. ; AJAYAN, P. M.. Tellurium-Assisted Low-Temperature Synthesis of $\mathrm{MoS}_{2}$ and WS $\mathrm{W}_{2}$ Monolayers. ACS Nano, 9(12):11658-11666, 2015.

[29] CHOI, S. H.; KIM, Y. J.; YANG, W. ; KIM, K. K.. Alkali Metal-Assisted Growth of Single-Layer Molybdenum Disulfide. J. Korean Phys. Soc., 74(11):1032-1038, 2019.

[30] HAN, W.; LIU, K.; YANG, S.; WANG, F.; SU, J. ; JIN, B.. Salt-assisted chemical vapor deposition of two-dimensional materials. SClENCE CHINA Chemistry, 2019.

[31] WANG, P.; LEI, J.; QU, J.; CAO, S.; JIANG, H.; HE, M.; SHI, H.; SUN, X.; GAO, B. ; LIU, W.. Mechanism of Alkali Metal CompoundPromoted Growth of Monolayer $\mathrm{MoS}_{2}$ : Eutectic Intermediates. Chem. Mater., 31(3):873-880, 2019.

[32] YANG, P.; ZOU, X.; ZHANG, Z.; HONG, M.; SHI, J.; CHEN, S.; SHU, J.; ZHAO, L.; JIANG, S.; ZHOU, X.; HUAN, Y.; XIE, C.; GAO, P.; CHEN, Q.; ZHANG, Q.; LIU, Z. ; ZHANG, Y.. Batch production of 6-inch uniform monolayer molybdenum disulfide catalyzed by sodium in glass. Nat. Commun., 9(1), 2018.

[33] CHEN, W.; ZHAO, J.; ZHANG, J.; GU, L.; YANG, Z.; LI, X.; YU, H.; ZHU, X.; YANG, R.; SHI, D.; LIN, X.; GUO, J.; BAI, X. ; ZHANG, G.. Oxygen-Assisted Chemical Vapor Deposition Growth of Large Single-Crystal and High-Quality Monolayer MoS 2. J. Am. Chem. Soc., 137(50):15632-15635, 2015.

[34] SHI, Y.; YANG, P.; JIANG, S.; ZHANG, Z.; HUAN, Y.; XIE, C.; HONG, M.; SHI, J. ; ZHANG, Y.. Na-assisted fast growth of large single-crystal $\mathrm{MoS}_{2}$ on sapphire. Nanotechnology, 30(3), 2019.

[35] SORKIN, V.; PAN, H.; SHI, H.; QUEK, S. Y. ; ZHANG, Y. W.. Nanoscale transition metal dichalcogenides: Structures, properties, and applications. Crit. Rev. Solid State Mater. Sci., 39(5):319-367, 2014.

[36] XU, K.; WANG, Z.; DU, X.; SAFDAR, M.; JIANG, C. ; HE, J.. Atomiclayer triangular $\mathrm{WSe}_{2}$ sheets: Synthesis and layer-dependent photoluminescence property. Nanotechnology, 24(46), 2013. 
[37] RUPPERT, C.; ASLAN, O. B. ; HEINZ, T. F.. Optical properties and band gap of single- and few-layer $\mathrm{MoTe}_{2}$ crystals. Nano Lett., 14(11):6231-6236, 2014.

[38] MAK, K.; LEE, C.; HONE, J.; SHAN, J. ; HEINZ, T.. Atomically Thin $\mathrm{MoS}_{2}$ : A New Direct-Gap Semiconductor. Phys. Rev. Lett., 105(13):136805, 2010.

[39] LIU, G. B.; SHAN, W. Y.; YAO, Y.; YAO, W. ; XIAO, D.. Three-band tight-binding model for monolayers of group-VIB transition metal dichalcogenides. Phys. Rev. B - Condens. Matter Mater. Phys., 88(8):1-11, 2013.

[40] KIM, E.; CHO, J.-W.; KIM, B. R.; THI, T.; NGUYEN, T.; NAM, Y.-H.; KIM, S.-K.; YOON, S.; KIM, Y. S.; LEE, J.-H. ; KIM, D.-W.. InterferenceEnhanced Broadband Absorption of Monolayer $\mathrm{MoS}_{2}$ on Sub$100 \mathrm{~nm}$ Thick $\mathrm{SiO}_{2} / \mathrm{Si}$ Substrates : Reflection and Transmission Phase Changes at Interfaces. Advanced Materials., 1701637:1-7, 2018.

[41] RUKELJ, Z.; ŠTRKALJ, A. ; DESPOJA, V.. Optical absorption and transmission in a molybdenum disulfide monolayer. Phys. Rev. B, 94(11):1-11, 2016.

[42] LI, Y.; DONG, N.; ZHANG, S.; ZHANG, X.; FENG, Y.; WANG, K.; ZHANG, L. ; WANG, J.. Giant two-photon absorption in monolayer $\mathbf{M o S}_{2}$. Laser Photonics Rev., 9(4):427-434, 2015.

[43] LEE, C.; YAN, H.; BRUS, L.; HEINZ, T.; HONE, J. ; RYU, S.. Anomalous lattice vibrations of single-and few-layer $\mathbf{M o S}_{2}$. ACS Nano, 4(5):2695-2700, 2010.

[44] LI, H.; ZHANG, Q.; YAP, C. C. R.; TAY, B. K.; EDWIN, T. H. T.; OLIVIER, A. ; BAILLARGEAT, D.. From bulk to monolayer $\mathbf{M o S}_{2}$ : Evolution of Raman scattering. Adv. Funct. Mater., 22(7):1385-1390, 2012.

[45] BERKDEMIR, A.; GUTIÉRREZ, H. R.; BOTELLO-MÉNDEZ, A. R.; PEREA-LÓPEZ, N.; ELÍAS, A. L.; CHIA, C. I.; WANG, B.; CRESPI, V. H.; LÓPEZ-URÍAS, F.; CHARLIER, J. C.; TERRONES, H. ; TERRONES, M.. Identification of individual and few layers of $\mathrm{WS}_{2}$ using Raman Spectroscopy. Sci. Rep., 3:1-8, 2013.

[46] MIGNUZZI, S.; POLLARD, A. J.; BONINI, N.; BRENNAN, B.; GILMORE, I. S.; PIMENTA, M. A.; RICHARDS, D. ; ROY, D.. Effect of disorder 
on Raman scattering of single-layer $\mathrm{MoS}_{2}$. Phys. Rev. B - Condens. Matter Mater. Phys., 91(19):1-7, 2015.

[47] AUTERE, A.; JUSSILA, H.; DAI, Y.; WANG, Y.; LIPSANEN, H. ; SUN, Z.. Nonlinear Optics with 2D Layered Materials. Adv. Mater., 30(24):1-24, 2018.

[48] WEN, X.; GONG, Z. ; LI, D.. Nonlinear optics of two-dimensional transition metal dichalcogenides. InfoMat, 1(3):317-337, 2019.

[49] WAgONER, G. A.; PERSANS, P. D.; VAN WAGENEN, E. A. ; KORENOWSKI, G. M.. Second-harmonic generation in molybdenum disulfide. J. Opt. Soc. Am. B, 15(3):1017, 1998.

[50] LI, Y.; RAO, Y.; MAK, K. F.; YOU, Y.; WANG, S.; DEAN, C. R. ; HEINZ, T. F.. Probing Symmetry Properties of Few-Layer $\mathrm{MoS}_{2}$ and h-BN by Optical Second-Harmonic Generation. Nano Letters, p. 3329-3333, 2013.

[51] MAlard, L. M.; AlenCAR, T. V.; BARBOZA, A. P. M.; MAK, K. F. ; DE PAULA, A. M.. Observation of intense second harmonic generation from $\mathrm{MoS}_{2}$ atomic crystals. Phys. Rev. B - Condens. Matter Mater. Phys., 87(20):1-5, 2013.

[52] KUMAR, N.; NAJMAEI, S.; CUI, Q.; CEBALLOS, F.; AJAYAN, P. M.; LOU, J. ; ZHAO, H.. Second harmonic microscopy of monolayer $\mathbf{M o S}_{2}$. Phys. Rev. B - Condens. Matter Mater. Phys., 87(16):1-6, 2013.

[53] WOODWARD, R. I.; MURRAY, R. T.; PHELAN, C. F.; DE OLIVEIRA, R. E. P.; RUNCORN, T. H.; KELLEHER, E. J. R.; LI, S.; DE OLIVEIRA, E. C.; FECHINE, G. J. M.; EDA, G. ; DE MATOS, C. J. S.. Characterization of the second- and third-order nonlinear optical susceptibilities of monolayer $\mathrm{MoS}_{2}$ using multiphoton microscopy. 2D Mater., 2016.

[54] CHEN, H.; CORBOLIOU, V.; SOLNTSEV, A. S.; CHOI, D.-Y.; VINCENTI, M. A.; DE CEGLIA, D.; DE ANGELIS, C.; LU, Y. ; NESHEV, D. N.. Enhanced second-harmonic generation from two-dimensional $\mathrm{MoSe}_{2}$ on a silicon waveguide. Light Sci. Appl., 6(10):e17060, 2017.

[55] LIN, X.; LIU, Y.; WANG, K.; WEI, C.; ZHANG, W.; YAN, Y.; LI, Y. J.; YAO, J. ; ZHAO, Y. S.. Two-Dimensional Pyramid-like $\mathbf{W S}_{2}$ Layered Structures for Highly Efficient Edge Second-Harmonic Generation. ACS Nano, 12(1):689-696, 2018. 
[56] HU, L.; WEI, D.; HUANG, X.; HU, L.; WEI, D. ; HUANG, X.. Second harmonic generation property of monolayer TMDCs and its potential application in producing terahertz radiation. THE JOURNAL OF CHEMICAL PHYSICS, 244701, 2017.

[57] JANISCH, C.; WANG, Y.; MA, D.; MEHTA, N.; ELÍAS, A. L.; PEREALÓPEZ, N.; TERRONES, M.; CRESPI, V. ; LIU, Z.. Extraordinary second harmonic generation in Tungsten disulfide monolayers. Sci. Rep., 4(c):1-5, 2014.

[58] SEYLER, K. L.; SCHAIBLEY, J. R.; GONG, P.; RIVERA, P.; JONES, A. M.; WU, S.; YAN, J.; MANDRUS, D. G.; YAO, W. ; XU, X.. Electrical control of second-harmonic generation in a $\mathrm{WSe}_{2}$ monolayer transistor. Nat. Nanotechnol., 10(5):407-411, 2015.

[59] RIBEIRO-SOARES, J.; JANISCH, C.; LIU, Z.; EĹIAS, A. L.; DRESSELHAUS, M. S.; TERRONES, M.; CANÇADO, L. G. ; JORIO, A.. Second harmonic generation in $\mathrm{WSe}_{2}$. 2D Mater., 2(4):1-6, 2015.

[60] PSILODIMITRAKOPOULOS, S.; MOUCHLIADIS, L.; PARADISANOS, I.; KOURMOULAKIS, G.; LEMONIS, A.; KIOSEOGLOU, G. ; STRATAKIS, E. Twist Angle mapping in layered $\mathrm{WS}_{2}$ by Polarization-Resolved Second Harmonic Generation. Sci. Rep., 9(1):1-11, 2019.

[61] TOH, R. J.; SOFER, Z.; LUXA, J.; SEDMIDUBSKÝ, D. ; PUMERA, M.. $3 \mathrm{R}$ phase of $\mathrm{MoS}_{2}$ and $\mathrm{WS}_{2}$ outperforms the corresponding $2 \mathrm{H}$ phase for hydrogen evolution. Chem. Commun., 53(21):3054-3057, 2017.

[62] HSU, W. T.; ZHAO, Z. A.; LI, L. J.; CHEN, C. H.; CHIU, M. H.; CHANG, P. S.; CHOU, Y. C. ; CHANG, W. H.. Second harmonic generation from artificially stacked transition metal dichalcogenide twisted bilayers. ACS Nano, 8(3):2951-2958, 2014.

[63] RADISAVLJEVIC, B.; RADENOVIC, A.; BRIVIO, J.; GIACOMETTI, V. ; KIS, A.. Single-layer $\mathrm{MoS}_{2}$ transistors. Nat. Nanotechnol., 6(3):147150, 2011.

[64] CHOI, W.; CHO, M. Y.; KONAR, A.; LEE, J. H.; CHA, G. B.; HONG, S. C.; KIM, S.; KIM, J.; JENA, D.; JOO, J. ; KIM, S.. High-detectivity multilayer $\mathrm{MoS}_{2}$ phototransistors with spectral response from ultraviolet to infrared. Adv. Mater., 24(43):5832-5836, 2012. 
[65] PARK, M. J.; MIN, J. K.; YI, S. G.; KIM, J. H.; OH, J. ; YOO, K. H.. Nearinfrared photodetectors utilizing $\mathrm{MoS}_{2}$-based heterojunctions. J. Appl. Phys., 118(4), 2015.

[66] WANG, W.; KLOTS, A.; PRASAI, D.; YANG, Y.; BOLOTIN, K. I. ; VALENTINE, J.. Hot Electron-Based Near-Infrared Photodetection Using Bilayer MoS $_{2}$. Nano Lett., 15(11):7440-7444, 2015.

[67] VAN DER ZANDE, A. M.; HUANG, P. Y.; CHENET, D. A.; BERKELBACH, T. C.; YOU, Y.; LEE, G.-H.; HEINZ, T. F.; REICHMAN, D. R.; MULLER, D. A. ; HONE, J. C.. Grains and grain boundaries in highly crystalline monolayer molybdenum disulphide. Nat. Mater., 12(6):554561, 2013.

[68] CHENG, R.; JIANG, S.; CHEN, Y.; LIU, Y.; WEISS, N.; CHENG, H.-C.; WU, H.; HUANG, Y. ; DUAN, X.. Few-layer molybdenum disulfide transistors and circuits for high-speed flexible electronics. Nat. Commun., 5:5143, 2014.

[69] LI, X.; YANG, L.; SI, M.; LI, S.; HUANG, M.; YE, P. ; WU, Y.. Performance potential and limit of $\mathrm{MoS}_{2}$ transistors. Adv. Mater., 27(9):1547-1552, 2015.

[70] AHN, J.-H.; PARKIN, W. M.; NAYLOR, C. H.; JOHNSON, A. T. C. ; DRNDIĆ, M.. Ambient effects on electrical characteristics of CVD-grown monolayer $\mathrm{MoS}_{2}$ field-effect transistors. Sci. Rep., $7(1): 4075,2017$.

[71] HSDS, T.; YIN, Z.; LI, H. H.; LI, H. H.; JIANG, L.; SHI, Y.; SUN, Y.; LU, G.; ZHANG, Q.; CHEN, X. ; ZHANG, H.. Single-layer $\mathbf{M o S}_{2}$ phototransistors. ACS Nano, .(1):74-80, 2012.

[72] THAKAR, K.; LODHA, S.. Optoelectronic and photonic devices based on transition metal dichalcogenides. Mater. Res. Express, 7(1), 2019.

[73] KUFER, D.; NIKITSKIY, I.; LASANTA, T.; NAVICKAITE, G.; KOPPENS, F. H. ; KONSTANTATOS, G.. Hybrid 2D-0D $\mathrm{MoS}_{2}-\mathrm{PbS}$ quantum dot photodetectors. Adv. Mater., 27(1):176-180, 2015.

[74] GONG, F.; FANG, H.; WANG, P.; SU, M.; LI, Q.; HO, J. C.; CHEN, X.; LU, W.; LIAO, L.; WANG, J. ; HU, W.. Visible to near-infrared photodetectors based on $\mathrm{MoS}_{2}$ vertical Schottky junctions. Nanotechnology, 28(48), 2017. 
[75] WANG, Y.; HUANG, X.; WU, D.; ZHUO, R.; WU, E.; JIA, C.; SHI, Z.; $X U$, T.; TIAN, Y. ; LI, X.. A room-temperature near-infrared photodetector based on a $\mathrm{MoS}_{2} / \mathrm{CdTe}$ p-n heterojunction with a broadband response up to $1700 \mathrm{~nm}$. J. Mater. Chem. C, 6(18):48614865, 2018.

[76] HWANG, I.; KIM, J. S.; CHO, S. H.; JEONG, B. ; PARK, C.. Flexible Vertical p-n Diode Photodetectors with Thin N-type $\mathrm{MoSe}_{2}$ Films Solution-Processed on Water Surfaces. ACS Appl. Mater. Interfaces, 10(40):34543-34552, 2018.

[77] ZHANG, B. Y.; LIU, T.; MENG, B.; LI, X.; LIANG, G.; HU, X. ; WANG, Q. J.. Broadband high photoresponse from pure monolayer graphene photodetector. Nat. Commun., 4(May):1811, 2013.

[78] HUO, N.; KONSTANTATOS, G.. Recent progress and future prospects of 2D-based photodetectors. Adv. Mater., 30(51):1-27, 2018.

[79] LOPEZ-SANCHEZ, O.; LEMBKE, D.; KAYCI, M.; RADENOVIC, A. ; KIS, A. Ultrasensitive photodetectors based on monolayer $\mathrm{MoS}_{2}$. Nat. Nanotechnol., 8(7):497-501, 2013.

[80] KOPPENS, F. H.; MUELLER, T.; AVOURIS, P.; FERRARI, A. C.; VITIELLO, M. S. ; POLINI, M.. Photodetectors based on graphene, other two-dimensional materials and hybrid systems. Nat. Nanotechnol., 9(10):780-793, 2014.

[81] CHAKRABORTY, B.; BERA, A.; MUTHU, D. V. S.; BHOWMICK, S.; WAGHMARE, U. V. ; SOOD, A. K.. Symmetry-dependent phonon renormalization in monolayer $\mathrm{MoS}_{2}$ transistor. Phys. Rev. B Condens. Matter Mater. Phys., 85(16):2-5, 2012.

[82] FLOWERS, P.; ROBINSON, W. R.. Chemistry 2e., volumen 1. Open stax, 2015.

[83] CHHOWALLA, M.; SHIN, H. S.; EDA, G.; LI, L. J.; LOH, K. P. ; ZHANG, $\mathrm{H}$.. The chemistry of two-dimensional layered transition metal dichalcogenide nanosheets. Nat. Chem., 5(4):263-275, 2013.

[84] WANG, Q. H.; KALANTAR-ZADEH, K.; KIS, A.; COLEMAN, J. N. ; STRANO, M. S.. Electronics and optoelectronics of twodimensional transition metal dichalcogenides. Nat. Nanotechnol., 7(11):699-712, 2012. 
[85] PODBEREZSKAYA, N. V.; MAGARILL, S. A.; PERVUKHINA, N. V. ; BORISOV, S. V.. Crystal chemistry of dichalcogenides $\mathbf{M X}_{2}$. J. Struct. Chem., 42(4):654-681, 2001.

[86] ZAFEIRATOS, S.. 2D Nanomaterials for Energy Applications. Elsevier, 1st ed. edition, 2019.

[87] ZHANG, Y. J.; OSHIDA, M. Y.; SUZUKI, R. ; IWASA, Y.. 2 D crystals of transition metal dichalcogenide and their iontronic functionalities. 2D Mater., 2(4):44004, 2015.

[88] RIBEIRO-SOARES, J.; ALMEIDA, R. M.; BARROS, E. B.; ARAUJO, P. T.; DRESSElHAUS, M. S.; CANÇADO, L. G. ; JORIO, A.. Group Theory analysis of phonons in two-dimensional Transition Metal Dichalcogenides. PHYSICAL REVIEW B, 115438, 2014.

[89] LIU, G. B.; XIAO, D.; YAO, Y.; XU, X. ; YAO, W.. Electronic structures and theoretical modelling of two-dimensional group-VIB transition metal dichalcogenides. Chem. Soc. Rev., 44(9):2643-2663, 2015.

[90] MOLINA-SÁNCHEZ, A.; WIRTZ, L.. Phonons in single-layer and few-layer $\mathbf{M o S}_{2}$ and $\mathbf{W S}_{2}$. Phys. Rev. B - Condens. Matter Mater. Phys., 84(15):1-8, 2011.

[91] YAN, J.; XIA, J.; WANG, X.; LIU, L.; KUO, J. L.; TAY, B. K.; CHEN, S.; ZHOU, W.; LIU, Z. ; SHEN, Z. X.. Stacking-Dependent Interlayer Coupling in Trilayer $\mathrm{MoS}_{2}$ with Broken Inversion Symmetry. Nano Lett., 15(12):8155-8161, 2015.

[92] YAN, A.; CHEN, W.; OPHUS, C.; CISTON, J.; LIN, Y.; PERSSON, K. ; ZETTL, A.. Identifying different stacking sequences in few-layer CVD-grown $\mathrm{MoS}_{2}$ by low-energy atomic-resolution scanning transmission electron microscopy. Phys. Rev. B, 93(4):1-5, 2016.

[93] SUZUKI, R.; SAKANO, M.; ZHANG, Y. J.; AKASHI, R.; MORIKAWA, D.; HARASAWA, A.; YAJI, K.; KURODA, K.; MIYAMOTO, K.; OKUDA, T.; ISHIZAKA, K.; ARITA, R. ; IWASA, Y.. Valley-dependent spin polarization in bulk $\mathrm{MoS}_{2}$ with broken inversion symmetry. Nat. Nanotechnol., 9(8):611-617, 2014.

[94] ZENG, Z.; SUN, X.; ZHANG, D.; ZHENG, W.; FAN, X.; HE, M.; XU, T.; SUN, L.; WANG, X. ; PAN, A.. Controlled Vapor Growth and 
Nonlinear Optical Applications of Large-Area 3R Phase $\mathbf{W S}_{2}$

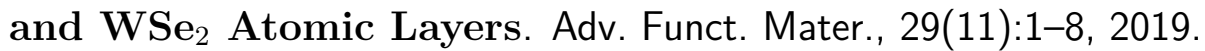

[95] JIANG, T.; LIU, H.; HUANG, D.; ZHANG, S.; LI, Y.; GONG, X.; SHEN, Y. R.; LIU, W. T. ; WU, S.. Valley and band structure engineering of folded $\mathrm{MoS}_{2}$ bilayers. Nat. Nanotechnol., 9(10):825-829, 2014.

[96] WIETING, T. J.; VERBLE, J. L.. Infrared and Raman studies of long-wavelength optical phonons in hexagonal $\mathrm{MoS}_{2}$. Phys. Rev. B, 3(12):4286-4292, 1971.

[97] HORZUM, S.; SAHIN, H.; CAHANGIROV, S.; CUDAZZO, P.; RUBIO, A.; SERIN, T. ; PEETERS, F. M.. Phonon softening and direct to indirect band gap crossover in strained single-layer $\mathrm{MoSe}_{2}$. Phys. Rev. B - Condens. Matter Mater. Phys., 87(12):1-5, 2013.

[98] CAI, Y.; LAN, J.; ZHANG, G. ; ZHANG, Y. W.. Lattice vibrational modes and phonon thermal conductivity of monolayer $\mathrm{MoS}_{2}$. Phys. Rev. B - Condens. Matter Mater. Phys., 89(3):1-8, 2014.

[99] ZHANG, X.; QIAO, X. F.; SHI, W.; WU, J. B.; JIANG, D. S. ; TAN, P. H.. Phonon and Raman scattering of two-dimensional transition metal dichalcogenides from monolayer, multilayer to bulk material. Chem. Soc. Rev., 44(9):2757-2785, 2015.

[100] ZHAO, W.; RIBEIRO, R. M. ; EDA, G.. Electronic structure and optical signatures of semiconducting transition metal dichalcogenide nanosheets. Acc. Chem. Res., 48(1):91-99, 2015.

[101] CHANG, C. H.; FAN, X.; LIN, S. H. ; KUO, J. L.. Orbital analysis of electronic structure and phonon dispersion in $\mathrm{MoS}_{2}, \mathrm{MoSe}_{2}$, $\mathbf{W S}_{2}$, and $\mathbf{W S e}_{2}$ monolayers under strain. Phys. Rev. B - Condens. Matter Mater. Phys., 88(19):1-9, 2013.

[102] SUN, Y.; WANG, D. ; SHUAI, Z.. Indirect-to-Direct Band Gap Crossover in Few-Layer Transition Metal Dichalcogenides: A Theoretical Prediction. J. Phys. Chem. C, 120(38):21866-21870, 2016.

[103] YAZYEV, O. V.; KIS, A.. $\mathrm{MoS}_{2}$ and semiconductors in the flatland. Mater. Today, 18(1):20-30, 2015.

[104] YAKOVKIN, I.. Dirac Cones in Graphene, Interlayer Interaction in Layered Materials, and the Band Gap in $\mathrm{MoS}_{2}$. Crystals, 6(11):143, 2016. 
[105] ELLIS, J. K.; LUCERO, M. J. ; SCUSERIA, G. E.. The indirect to direct band gap transition in multilayered $\mathrm{MoS}_{2}$ as predicted by screened hybrid density functional theory. Appl. Phys. Lett., 99(26):261908, 2011.

[106] TUSCHEL, D.. Raman Spectroscopy and Imaging of LowEnergy Phonons Raman. Spectroscopy Solutions for Materials Analysis, 30(September), 2015.

[107] EDA, G.; MAIER, S. A.. Two-dimensional crystals: Managing light for optoelectronics. ACS Nano, 7(7):5660-5665, 2013.

[108] PIPER, J. R.; FAN, S.. Broadband Absorption Enhancement in Solar Cells with an Atomically Thin Active Layer. ACS Photonics, 3(4):571-577, 2016.

[109] QIU, D. Y.; DA JORNADA, F. H. ; LOUIE, S. G.. Optical spectrum of $\mathrm{MoS}_{2}$ : Many-body effects and diversity of exciton states. Phys. Rev. Lett., 111(21):1-5, 2013.

[110] SIE, E. J.; FRENZEL, A. J.; LEE, Y. H.; KONG, J. ; GEDIK, N.. Intervalley biexcitons and many-body effects in monolayer $\mathrm{MoS}_{2}$. Phys. Rev. B - Condens. Matter Mater. Phys., 92(12):1-9, 2015.

[111] BELluS, M. Z.; CEBALlOS, F.; CHIU, H. Y. ; ZHAO, H.. Tightly Bound Trions in Transition Metal Dichalcogenide Heterostructures. ACS Nano, 9(6):6459-6464, 2015.

[112] CAO, T.; WANG, G.; HAN, W.; YE, H.; ZHU, C.; SHI, J.; NIU, Q.; TAN, P.; WANG, E.; LIU, B. ; FENG, J.. Valley-selective circular dichroism of monolayer molybdenum disulphide. Nat. Commun., 3(May), 2012.

[113] LIU, Y.; GAO, Y.; ZHANG, S.; HE, J.; YU, J. ; LIU, Z.. Valleytronics in transition metal dichalcogenides materials. Nano Res., 12(11):2695-2711, 2019.

[114] VERHAGEN, T.; GUERRA, V. L.; HAIDER, G.; KALBAC, M. ; VEJPRAVOVA, J.. Towards the evaluation of defects in $\mathrm{MoS}_{2}$ using cryogenic photoluminescence spectroscopy. Nanoscale, 12(5):30193028, 2020.

[115] MAK, K. F.; HE, K.; LEE, C.; LEE, G. H.; HONE, J.; HEINZ, T. F. ; SHAN, J.. Tightly bound trions in monolayer $\mathbf{M o S}_{2}$. Nat. Mater., 12(3):207-211, 2013. 
[116] NEWAZ, A. K. M.; PRASAI, D.; ZIEGLER, J. I.; CAUDEL, D.; ROBINSON, S.; HAGLUND, R. F. ; BOLOTIN, K. I.. Electrical Control of Optical Properties of Monolayer $\mathbf{M o S}_{2}$. Solid State Communications., 2:1-5, 2012.

[117] PIMENTA, M. A.; DEL CORRO, E.; CARVALHO, B. R.; FANTINI, C. ; MALARD, L. M.. Comparative Study of Raman Spectroscopy in Graphene and $\mathrm{MoS}_{2}$-type Transition Metal Dichalcogenides. Acc. Chem. Res., 48(1):41-47, 2015.

[118] TERRONES, H.; DEL CORRO, E.; FENG, S.; POUMIROL, J. M.; RHODES, D.; SMIRNOV, D.; PRADHAN, N. R.; LIN, Z.; NGUYEN, M. A.; ELIIAS, A. L.; MALLOUK, T. E.; BALICAS, L.; PIMENTA, M. A. ; TERRONES, M.. New First Order Raman-active Modes in Few Layered Transition Metal Dichalcogenides. Sci. Rep., 4:1-9, 2014.

[119] ZHU, S.; WANG, Q.. A simple method for understanding the triangular growth patterns of transition metal dichalcogenide sheets. AIP Adv., 5(10), 2015.

[120] CAO, D.; SHEN, T.; LIANG, P.; CHEN, X. ; SHU, H.. Role of chemical potential in flake shape and edge properties of monolayer $\mathrm{MoS}_{2}$. J. Phys. Chem. C, 119(8):4294-4301, 2015.

[121] WANG, Y.; XIAO, J.; YANG, S.; WANG, Y. ; ZHANG, X.. Second harmonic generation spectroscopy on two-dimensional materials. Opt. Mater. Express, 9(3):1136, 2019.

[122] SÄYNÄTJOKI, A.; KARVONEN, L.; ROSTAMI, H.; AUTERE, A.; MEHRAVAR, S.; LOMBARDO, A.; NORWOOD, R. A.; HASAN, T.; PEYGHAMBARIAN, N.; LIPSANEN, H.; KIEU, K.; FERRARI, A. C.; POLINI, M. ; SUN, Z.. Ultra-strong nonlinear optical processes and trigonal warping in $\mathrm{MoS}_{2}$ layers. Nat. Commun., 8(1):1-8, 2017.

[123] YU, H.; TALUKDAR, D.; XU, W.; KHURGIN, J. B. ; XIONG, Q.. ChargeInduced Second-Harmonic Generation in Bilayer $\mathrm{WSe}_{2}$. Nano Lett., 15(8):5653-5657, 2015.

[124] WANG, R.; CHIEN, H. C.; KUMAR, J.; KUMAR, N.; CHIU, H. Y. ; ZHAO, $\mathrm{H}$.. Third-harmonic generation in ultrathin films of $\mathrm{MoS}_{2}$. ACS Appl. Mater. Interfaces, 6(1):314-318, 2014. 
[125] LIU, H.; ANTWI, K. K.; CHUA, S. ; CHI, D.. Vapor-phase growth and characterization of $\mathrm{Mo}_{1-x} \mathrm{~W}_{x} \mathrm{~S}_{2}(0 \leq \mathrm{x} \leq 1)$ atomic layers on 2-inch sapphire substrates. Nanoscale, 6(1):624-629, 2014.

[126] FU, Q.; YANG, L.; WANG, W.; HAN, A.; HUANG, J.; DU, P.; FAN, Z.; ZHANG, J. ; XIANG, B.. Synthesis and Enhanced Electrochemical Catalytic Performance of Monolayer $\mathbf{W S}_{2(1-x)} \mathbf{S e}_{2 x}$ with a Tunable Band Gap. Adv. Mater., 27(32):4732-4738, 2015.

[127] TONGAY, S.; NARANG, D. S.; KANG, J.; FAN, W.; KO, C.; LUCE, A. V.; WANG, K. X.; SUH, J.; PATEL, K. D.; PATHAK, V. M.; LI, J. ; WU, J.. Two-dimensional semiconductor alloys: Monolayer $\mathbf{M o}_{1-x} \mathbf{W}_{x} \mathbf{S e}_{2}$. Appl. Phys. Lett., 104(1), 2014.

[128] LIN, Y. K.; CHEN, R. S.; CHOU, T. C.; LEE, Y. H.; CHEN, Y. F.; CHEN, K. H. ; CHEN, L. C.. Thickness-Dependent Binding Energy Shift in Few-Layer $\mathrm{MoS}_{2}$ Grown by Chemical Vapor Deposition. ACS Appl. Mater. Interfaces, 8(34):22637-22646, 2016.

[129] SYARI'ATI, A.; KUMAR, S.; ZAHID, A.; ALI EL YUMIN, A.; YE, J. ; RUDOLF, P.. Photoemission spectroscopy study of structural defects in molybdenum disulfide $\left(\mathrm{MoS}_{2}\right)$ grown by chemical vapor deposition (CVD). Chem. Commun., 55(70):10384-10387, 2019.

[130] LI, X.; CAI, W.; AN, J.; KIM, S.; NAH, J.; YANG, D.; PINER, R.; VELAMAKANNI, A.; JUNG, I.; TUTUC, E.; BANERJEE, S. K.; COLOMBO, L. ; RUOFF, R. S.. Large-area synthesis of high-quality and uniform graphene films on copper foils. Science (80-. )., 324(5932):1312-1314, 2009.

[131] ZHU, H. W.; XU, C. L.; WU, D. H.; WEI, B. Q.; VAJTAI, R. ; AJAYAN, P. M.. Direct synthesis of long single-walled carbon nanotube strands. Science (80-. )., 296(5569):884-886, 2002.

[132] SONG, L.; CI, L.; LU, H.; SOROKIN, P. B.; JIN, C.; NI, J.; KVASHNIN, A. G.; KVASHNIN, D. G.; LOU, J.; YAKOBSON, B. I. ; AJAYAN, P. M.. Large scale growth and characterization of atomic hexagonal boron nitride layers. Nano Lett., 10(8):3209-3215, 2010.

[133] KALITA, G.; TANEMURA, M.. Fundamentals of Chemical Vapor Deposited Graphene and Emerging Applications. Graphene Mater. - Adv. Appl., 2017. 
[134] YU, J.; LI, J.; ZHANG, W. ; CHANG, H.. Synthesis of high quality two-dimensional materials via chemical vapor deposition. Chem. Sci., 6(12):6705-6716, 2015.

[135] YOU, J.; HOSSAIN, M. D. ; LUO, Z.. Synthesis of 2D transition metal dichalcogenides by chemical vapor deposition with controlled layer number and morphology. Nano Converg., 5(1), 2018.

[136] ZHANG, Y.; YAO, Y.; SENDEKU, M. G.; YIN, L.; ZHAN, X.; WANG, F.; WANG, Z. ; HE, J.. Recent Progress in CVD Growth of 2D Transition Metal Dichalcogenides and Related Heterostructures. Adv. Mater., 31(41):1-30, 2019.

[137] CAI, Z.; LIU, B.; ZOU, X. ; CHENG, H.-M.. Chemical Vapor Deposition Growth and Applications of Two-Dimensional Materials and Their Heterostructures. Chem. Rev., 118:acs.chemrev.7b00536, 2018.

[138] ZHANG, T.; FU, L.. Controllable Chemical Vapor Deposition Growth of Two-Dimensional Heterostructures. Chem, 4(4):671689, 2018.

[139] YIN, G.; ZHU, D.; LV, D.; HASHEMI, A.; FEI, Z.; LIN, F.; KRASHENINNIKOV, A. V.; ZHANG, Z.; KOMSA, H. P. ; JIN, C.. Hydrogenassisted post-growth substitution of tellurium into molybdenum disulfide monolayers with tunable compositions. Nanotechnology, 29(14), 2018.

[140] .. https://www.shutterstock.com/editorial/imageeditorial/education-2557517a. ., .

[141] C.V. RAMAN. The Raman Effect: Investigation of molecular structure by light scattering. Trans. Faraday Soc., 25(September):781-792, 1929.

[142] FERRARO, J. R.; NAKAMOTO, K. ; BROWN, C. W.. Introductory Raman Spectroscopy: Second Edition. Elsevier, 2003.

[143] DONG, Z.; XU, H.; LIANG, F.; LUO, C.; WANG, C.; CAO, Z. Y.; CHEN, X. J.; ZHANG, J. ; WU, X.. Raman characterization on twodimensional materials-based thermoelectricity. Molecules, 24(1), 2019. 
[144] TUSCHEL, D.. Photoluminescence spectroscopy using a Raman spectrometer. Spectroscopy Solutions for Materials Analysis, 31(9):1421, 2016.

[145] BAYKARA, M. Z.; SCHWARZ, U. D.. Atomic force microscopy: Methods and applications. Elsevier Inc., 3 edition, 2016.

[146] .. https://upload.wikimedia.org/wikipedia/commons/5/5e/AFMsetup. ., jpg.

[147] NT-MDT. https://www.ntmdt-si.com/products/afm-ramannano-ir-systems/ntegra-spectra-ii. ., .

[148] STOJILOVIC, N.. Why can't we see hydrogen in X-ray photoelectron spectroscopy? J. Chem. Educ., 89(10):1331-1332, 2012.

[149] ORTEN, D.. <Einstein-1905-Annalen_der_Physik.pdf > .., .(.).., 1905.

[150] HUFNER. Photoelectron Spectroscopy. Springer-Verlag Berlin Heidelberg $\mathrm{GmbH}, 2003$.

[151] WEIMER, J. J.. X-RAY PHOTOELECTRON SPECTROSCOPY. Wiley Online Library, p. 1493-1535, 2012.

[152] .. https://upload.wikimedia.org/wikipedia/commons/f/f2. ., /System2.gif.

[153] FRANKEN, P. A.; HILL, A. E.; PETERS, C. W. ; WEINREICH, G.. Generation of optical harmonics. Phys. Rev. Lett., 7(4):118-119, 1961.

[154] CHEN, H.; TANG, Y.; JIANG, T. ; LI, G.. Nonlinear nanophotonics with 2D transition metal dichalcogenides, volumen 1-5. Elsevier Ltd., 2019.

[155] SUÑÉ, G. R.. Electron beam lithography for Nanofabrication. Nanomater. Nanostructures, Nanotechnologie, .(January):1-20, 2008.

[156] RODDARO, S.; PINGUE, P.; PIAZZA, V.; PELLEGRINI, V. ; BELTRAM, F.. The optical visibility of graphene: Interference colors of ultrathin graphite on $\mathrm{SiO}_{2}$. Nano Lett., 7(9):2707-2710, 2007.

[157] NI, Z. H.; WANG, H. M.; KASIM, J.; FAN, H. M.; YU, T.; WU, Y. H.; FENG, Y. P. ; SHEN, Z. X.. Graphene Thickness Determination Using Reflection and Contrast Spectroscopy. Nano Latters, 2007. 
[158] LI, H.; WU, J.; HUANG, X.; LU, G.; YANG, J.; LU, X.; XIONG, Q. ; ZHANG, H.. Rapid and Reliable Thickness Identification of TwoDimensional Nanosheets Using Optical Microscopy. ACS Nano, .(Xx), 2013.

[159] SHI, B.; ZHOU, D.; FANG, S.; DJEBBI, K.; FENG, S.; ZHAO, H.; TLILI, C. ; WANG, D.. Facile and controllable synthesis of large-area monolayer $\mathrm{WS}_{2}$ flakes based on $\mathrm{WO}_{3}$ precursor drop-casted substrates by chemical vapor deposition. Nanomaterials, 9(4), 2019.

[160] MARTIN I. PECH-CANUL; RAVINDRA, N. M.. Semiconductors Synthesis,properties and applications. Springer, 2019.

[161] LEE, J.-U.; PARK, J.; SON, Y.-W. ; CHEONG, H.. Anomalous excitonic resonance Raman effects in few-layered $\mathrm{MoS}_{2}$. Nanoscale, 7(7):3229-3236, 2015.

[162] CARVALHO, B. R.; WANG, Y.; MIGNUZZI, S.; ROY, D.; TERRONES, M.; FANTINI, C.; CRESPI, V. H.; MALARD, L. M. ; PIMENTA, M. A.. Intervalley scattering by acoustic phonons in two-dimensional $\mathrm{MoS}_{2}$ revealed by double-resonance Raman spectroscopy. Nature Communications, 8(1):14670, 2017.

[163] CHEN, J. M.; WANG, C. S.. Second order Raman spectrum of $\mathrm{MoS}_{2}$. Solid State Commun., 14(9):857-860, 1974.

[164] CHAKRABORTY, B.; MATTE, H. S. S. R.; SOOD, A. K. ; RAO, C. N. R.. Layer-dependent resonant Raman scattering of a few layer $\mathrm{MoS}_{2}$. J. Raman Spectrosc., 44(1):92-96, 2013.

[165] CARVALHO, B. R.; WANG, Y.; MIGNUZZI, S.; ROY, D.; TERRONES, M.; FANTINI, C.; CRESPI, V. H.; MALARD, L. M. ; PIMENTA, M. A.. Intervalley scattering by acoustic phonons in two-dimensional MoS2revealed by double-resonance Raman spectroscopy. Nat. Commun., 8:1-8, 2017.

[166] XIAO, S.; XIAO, P.; ZHANG, X.; YAN, D.; GU, X.; QIN, F.; NI, Z.; HAN, Z. J. ; OSTRIKOV, K. K.. Atomic-layer soft plasma etching of $\mathbf{M o S}_{2}$. Sci. Rep., 6(December 2015):1-8, 2016.

[167] SHI, W.; LIN, M. L.; TAN, Q. H.; QIAO, X. F.; ZHANG, J. ; TAN, P. H.. Raman and photoluminescence spectra of two-dimensional nanocrystallites of monolayer $\mathbf{W S}_{2}$ and $\mathbf{W S e}_{2}$. 2D Mater., 3(2):110, 2016. 
[168] GAUR, A. P. S.; SAHOO, S.; SCOTT, J. F. ; KATIYAR, R. S.. Electron - Phonon interaction and double-resonance Raman studies in monolayer WS $\mathbf{W}_{2}$. J. Phys. Chem. C, 119(9):5146-5151, 2015.

[169] MOLAS, M. R.; NOGAJEWSKI, K.; POTEMSKI, M. ; BABIŃSKI, A.. Raman scattering excitation spectroscopy of monolayer $\mathbf{W S}_{2}$. Sci. Rep., 7(1):1-8, 2017.

[170] KIM, M. S.; NAM, G.; PARK, S.; KIM, H.; HAN, G. H.; LEE, J.; DHAKAL, K. P.; LEEM, J. Y.; LEE, Y. H. ; KIM, J.. Photoluminescence wavelength variation of monolayer $\mathrm{MoS}_{2}$ by oxygen plasma treatment. Thin Solid Films, 590:318-323, 2015.

[171] KOZAWA, D.; KUMAR, R.; CARVALHO, A.; KUMAR AMARA, K.; ZHAO, W.; WANG, S.; TOH, M.; RIBEIRO, R. M.; CASTRO NETO, A. H.; MATSUDA, K. ; EDA, G.. Photocarrier relaxation pathway in twodimensional semiconducting transition metal dichalcogenides. Nat. Commun., 5:1-7, 2014.

[172] GUTIÉRREZ, H. R.; PEREA-LÓPEZ, N.; ELÍAS, A. L.; BERKDEMIR, A.; WANG, B.; LV, R.; LÓPEZ-URÍAS, F.; CRESPI, V. H.; TERRONES, H. ; TERRONES, M.. Extraordinary room-temperature photoluminescence in triangular $\mathbf{W S}_{2}$ monolayers. Nano Lett., 13(8):3447-3454, 2013.

[173] MAK, K. F.; LEE, C.; HONE, J.; SHAN, J. ; HEINZ, T. F.. Atomically thin $\mathrm{MoS}_{2}$ : A new direct-gap semiconductor. Phys. Rev. Lett., 105(13):2-5, 2010.

[174] ZHOU, B.; YANG, L.; CHEN, F.; XU, M.; WU, T.; WU, G.; CHEN, X. ; FENG, D.. Evolution of Electronic Structure in Atomically Thin Sheets of $\mathbf{W S}_{2}$ and $\mathbf{W S e}_{2}$. ACS Nano, 7(Xx):1, 791-797, 2013.

[175] BOND, B. D.; JACOBS, W. M.. The Thermal Decomposition of Sodium Nitrate. Journal Chemical Society (A), p. 2-5, 1966.

[176] BRUIX, A.; FÜCHTBAUER, H. G.; TUXEN, A. K.; WALTON, A. S.; ANDERSEN, M.; PORSGAARD, S.; BESENBACHER, F.; HAMMER, B. ; LAURITSEN, J. V.. In Situ Detection of Active Edge Sites in Single-Layer $\mathbf{M o S}_{2}$ Catalysts. ACS Nano, 9(9):9322-9330, 2015.

[177] GIANNOZZI, P.; BARONI, S.; BONINI, N.; CALANDRA, M.; CAR, R.; CAVAZZONI, C.; CERESOLI, D.; CHIAROTTI, G. L.; COCOCCIONI, M.; 
DABO, I.; CORSO, A. D.; DE GIRONCOLI, S.; FABRIS, S.; FRATESI, G.; GEBAUER, R.; GERSTMANN, U.; GOUGOUSSIS, C.; KOKALJ, A.; LAZZERI, M.; MARTIN-SAMOS, L.; MARZARI, N.; MAURI, F.; MAZZARELLO, R.; PAOLINI, S.; PASQUARELLO, A.; PAULATTO, L.; SBRACCIA, C.; SCANDOLO, S.; SCLAUZERO, G.; SEITSONEN, A. P.; SMOGUNOV, A.; UMARI, P. ; WENTZCOVITCH, R. M.. QUANTUM ESPRESSO: a modular and open-source software project for quantum simulations of materials. Journal of Physics: Condensed Matter, 21(39):395502, sep 2009.

[178] CAROZO, V.; WANG, Y.; FUJISAWA, K.; CARVALHO, B. R.; MCCREARY, A.; FENG, S.; LIN, Z.; ZHOU, C.; PEREA-LÓPEZ, N.; ELÍAS, A. L.; KABIUS, B.; CRESPI, V. H. ; TERRONES, M.. Optical identification of sulfur vacancies: Bound excitons at the edges of monolayer tungsten disulfide. Sci. Adv., 3(4):e1602813, 2017.

[179] ROSSI, T. P.; WINTHER, K. T.; JACOBSEN, K. W.; NIEMINEN, R. M.; PUSKA, M. J. ; THYGESEN, K. S.. Effect of edge plasmons on the optical properties of $\mathrm{MoS}_{2}$ monolayer flakes. Phys. Rev. B, 96(15), 2017.

[180] LI, H.; CONTRYMAN, A. W.; QIAN, X.; ARDAKANI, S. M.; GONG, Y.; WANG, X.; WEISSE, J. M.; LEE, C. H.; ZHAO, J.; AJAYAN, P. M.; LI, J.; MANOHARAN, H. C. ; ZHENG, X.. Optoelectronic crystal of artificial atoms in strain-textured molybdenum disulphide. Nat. Commun., 6(May):6-11, 2015.

[181] CHANG, H. Y.; YOGEESH, M. N.; GHOSH, R.; RAI, A.; SANNE, A.; YANG, S.; LU, N.; BANERJEE, S. K. ; AKINWANDE, D.. Large-Area Monolayer $\mathrm{MoS}_{2}$ for Flexible Low-Power RF Nanoelectronics in the GHz Regime. Adv. Mater., 28(9):1818-1823, 2016.

[182] HENRIE, J.; KELLIS, S.; SCHULTZ, S. M. ; HAWKINS, A.. Electronic color charts for dielectric films on silicon. Opt. Express, 12(7):1464, 2004.

[183] YOON, D.; MOON, H.; SON, Y. W.; CHOI, J. S.; PARK, B. H.; CHA, Y. H.; KIM, Y. D. ; CHEONG, H.. Interference effect on Raman spectrum of graphene on $\mathrm{SiO}_{2} / \mathrm{Si}$. Phys. Rev. B - Condens. Matter Mater. Phys., 80(12):1-21, 2009.

[184] SHOUTE, L. C.; BERGREN, A. J.; MAHMOUD, A. M.; HARRIS, K. D. ; MCCREERY, R. L.. Optical interference effects in the design 
of substrates for surface-enhanced raman spectroscopy. Appl. Spectrosc., 63(2):133-140, 2009.

[185] LIANG, L.; MEUNIER, V.. First-principles Raman spectra of $\mathrm{MoS}_{2}$, WS $_{2}$ and their heterostructures. Nanoscale, 6(10):5394-5401, 2014.

[186] QIAO, S.; YANG, H.; BAI, Z.; PENG, G. ; ZHANG, X.. Identifying the number of $\mathrm{WS}_{2}$ layers via Raman and photoluminescence spectrum. Advances in Engineering Research, 141(Icmmcce):1408-1413, 2017.

[187] YUANZHENG, L.; XINSHU, L.; TONG, Y.; GUOCHUN, Y.; HEYU, C.; CEN, Z.; QIUSHI, F.; JIANGANG, M.; WEIZHEN, L.; HAIYANG, X.; YICHUN, L. ; XINFENG, L.. Accurate identification of layer number for few-layer $\mathrm{WS}_{2}$ and $\mathrm{WSe}_{2}$ via spectroscopic study. Nanotechnology, 29(12):124001, 2018.

[188] MILTOS MARAGKAKIS, G.; PSILODIMITRAKOPOULOS, S.; MOUCHLIADIS, L.; PARADISANOS, I.; LEMONIS, A.; KIOSEOGLOU, G. ; STRATAKIS, E.. Imaging the crystal orientation of $2 \mathrm{D}$ transition metal dichalcogenides using polarization-resolved secondharmonic generation. Opto-Electronic Adv., 2(11):19002601-19002608, 2019.

[189] QI, Z.; CAO, P. ; PARK, H. S.. Density functional theory calculation of edge stresses in monolayer $\mathbf{M o S}_{2}$. J. Appl. Phys., 114(16):1-5, 2013.

[190] BOYD, R.. R. Boyd, Nonlinear Optics (Academic, London, 2008). Elsevier Inc., 2008.

[191] VIANNA, P. G.; HAMZA SAFEER, S.; ORE, A. S.; GORDO, V. O.; CARVALHO, I. C.; CAROZO, V. ; DE MATOS, C. J.. Synthesis and Characterization of $\mathrm{MoS}_{2} / \mathrm{WS}_{2}$ Heterostructures by Second Harmonic Generation. 2019 Sbfot. Int. Opt. Photonics Conf. Sbfot. IOPC 2019, .:4-7, 2019.

[192] SAGAR, A.; BALASUBRAMANIAN, K.; BURGHARD, M.; KERN, K. ; SORDAN, R.. Polymer-electrolyte gated graphene transistors for analog and digital phase detection. Appl. Phys. Lett., 99(4):20092012, 2011.

[193] DAS, A.; PISANA, S.; CHAKRABORTY, B.; PISCANEC, S.; SAHA, S. K.; WAGHMARE, U. V.; NOVOSELOV, K. S.; KRISHNAMURTHY, H. R.; 
GEIM, A. K.; FERRARI, A. C. ; SOOD, A. K.. Monitoring dopants by Raman scattering in an electrochemically top-gated graphene transistor. Nat. Nanotechnol., 3(4):210-215, 2008.

[194] MAK, K. F.; LUI, C. H.; SHAN, J. ; HEINZ, T. F.. Observation of an electric-field-induced band gap in bilayer graphene by infrared spectroscopy. Phys. Rev. Lett., 102(25):100-103, 2009.

[195] EFETOV, D. K.; KIM, P.. Controlling electron-phonon interactions in graphene at ultrahigh carrier densities. Phys. Rev. Lett., 105(25):2-5, 2010.

[196] YAN, J.; HENRIKSEN, E. A.; KIM, P. ; PINCZUK, A.. Observation of anomalous phonon softening in bilayer graphene. Phys. Rev. Lett., 101(13):26-29, 2008.

[197] SCHWIERZ, F.; PEZOLDT, J. ; GRANZNER, R.. Two-dimensional materials and their prospects in transistor electronics. Nanoscale, 7(18):8261-8283, 2015.

[198] TONG, X.; ASHALLEY, E.; LIN, F.; LI, H. ; WANG, Z. M.. Advances in $\mathrm{MoS}_{2}$-Based Field Effect Transistors (FETs). Nano-Micro Lett., 7(3):203-218, 2015.

[199] DHAR, S.; PATTANAIK, M.. Layered TMDFETS for Nano Devices: A Short Review. J. At. Mol. Condens. Nano Phys., 6(3):191-198, 2019.

[200] BENKA, S. G.. Two-dimensional atomic crystals. Phys. Today, 58(9):9-9, 2005.

[201] ȘAR, H.; ÖZDEN, A.; YORULMAZ, B.; SEVIK, C.; KOSKU PERKGOZ, N. ; AY, F.. A comparative device performance assesment of CVD grown $\mathrm{MoS}_{2}$ and $\mathbf{W S}_{2}$ monolayers. J. Mater. Sci. Mater. Electron., 29(10):8785-8792, 2018.

[202] GONG, Y.; LIN, J.; WANG, X.; SHI, G.; LEI, S.; LIN, Z.; ZOU, X.; YE, G.; VAJTAI, R.; YAKOBSON, B. I.; TERRONES, H.; TERRONES, M.; TAY, B. K.; LOU, J.; PANTELIDES, S. T.; LIU, Z.; ZHOU, W. ; AJAYAN, P. M.. Vertical and in-plane heterostructures from $\mathrm{WS}_{2} / \mathrm{MoS}_{2}$ monolayers. Nat. Mater., 13(12):1135-1142, 2014.

[203] YAN, J.; ZHANG, Y.; KIM, P. ; PINCZUK, A.. Electric field effect tuning of electron-phonon coupling in graphene. Phys. Rev. Lett., 98(16):1-4, 2007. 
[204] MCDONNELL, S.; ADDOU, R.; BUIE, C.; WALLACE, R. M. ; HINKLE, C. L.. Defect-DOMINATED DOPING and CONTACT RESISTANCE in $\mathrm{MoS}_{2}$. ACS Nano, 8(3):2880-2888, 2014.

[205] LEE, C.; JEONG, B. G.; YUN, S. J.; LEE, Y. H.; LEE, S. M. ; JEONG, M. S.. Unveiling Defect-Related Raman Mode of Monolayer $\mathrm{WS}_{2}$ via Tip-Enhanced Resonance Raman Scattering. ACS Nano, 12(10):9982-9990, 2018. 